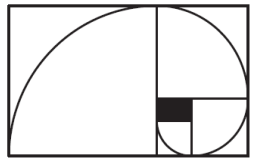

\title{
Simplicité des groupes de transformations de surfaces
}

\author{
Abed Bounemoura
}

\begin{abstract}
The study of algebraic properties of groups of transformations of a manifold gives rise to an interplay between different areas of mathematics such as topology, geometry, dynamical systems and foliation theory. This volume is devoted to the question of simplicity of such groups, and we will mainly restrict our attention to the case where the manifold is a surface. In the first chapter, we will show that the identity component of the group of homeomorphisms of a closed surface is simple. This will lead us to the case of diffeomorphisms, and in the second chapter, we will give the complete proof of the Epstein-Herman-Mather-Thurston theorem stating that the group of $C^{\infty}$-diffeomorphisms isotopic to the identity is also simple. We will also review the link with classifying spaces for foliations, and a result of Mather showing that the theorem remains true for $C^{k}$-diffeomorphisms, provided that $k$ is different from $n+1$, where $n$ is the dimension of the manifold. The last two chapters deal with conservative homeomorphisms and diffeomorphisms, by which we mean preserving a measure or a smooth volume or symplectic form. In those cases, there is a generalized rotation number showing that the associated groups cannot be simple. For conservative diffeomorphisms, the situation is well understood thanks to the work of Banyaga, but this is definitely not the case for conservative homeomorphisms of surfaces, and we will present some open problems in this direction as well as different attempts to solve them.
\end{abstract}

2000 Mathematics Subject Classification: 58D05, 37E30. 



\section{Remerciements}

Ce texte est issu d'un stage effectué sous la direction de Frédéric Le Roux à l'université d'Orsay. Je le remercie très profondément de m'avoir fait confiance en acceptant de me guider sur ce travail, ainsi que de la gentillesse et de la disponibilité dont il a fait preuve. Sans ses explications, ses nombreuses améliorations et ses multiples relectures approfondies, la rédaction de ce manuscrit n'aurait tout simplement pas été possible. Il m'a également beaucoup aidé à la fin pour terminer la rédaction de l'introduction alors que j'étais en panne. Je me permets encore de lui exprimer toute ma gratitude.

Je remercie également Étienne Ghys pour l'intérêt qu'il a porté à ce travail, François Béguin, Sylvain Crovisier, Pierre Py et Jérôme Buzzi pour leurs relectures et leurs commentaires sur certaines parties de ce texte, ainsi que tous mes amis doctorants d'Orsay et de Chevaleret. 



\section{Table des matières}

$\begin{array}{ll}\text { Introduction } & 7\end{array}$

1 Homéomorphismes de surfaces 11

1.1 Simplicité du groupe $\operatorname{Homeo}^{0}(M)$. . . . . . . . . . 11

1.2 Simplicité du groupe $\mathrm{Homeo}_{0}(M) \ldots \ldots \ldots \ldots$

2 Difféomorphismes de surfaces 22

2.1 Simplicité du groupe $\operatorname{Diff} f_{0}^{\infty}\left(\mathbb{T}^{2}\right) \ldots \ldots \ldots \ldots \ldots \ldots$

2.2 Simplicité du groupe Dif $f_{0}^{\infty}(M) \ldots \ldots \ldots \ldots$

2.3 Feuilletages et espaces classifiants . . . . . . . . . . . . . 43

2.4 Simplicité de $\operatorname{Dif} f_{0}^{r}(M)$ pour $r \neq 3 \ldots \ldots \ldots \ldots$

3 Difféomorphismes conservatifs $\quad 62$

3.1 Premier invariant de Calabi . . . . . . . . . . . . . . 63

3.2 Difféomorphismes hamiltoniens . . . . . . . . . . . . 72

3.3 Second invariant de Calabi . . . . . . . . . . . . . . . 81

3.4 Théorème de simplicité de Banyaga . . . . . . . . . . . . 84

4 Homéomorphismes conservatifs $\quad 98$

4.1 Approche de Fathi . . . . . . . . . . . . . . . . . . . 99

4.2 Approche de Gambaudo et Ghys . . . . . . . . . . . 105

4.3 Distance de Hofer . . . . . . . . . . . . . . . . . . . . . 111

4.4 Approche de $\mathrm{Oh} \ldots \ldots \ldots \ldots$

$\begin{array}{ll}\text { A Théorème de Schoenflies } & 125\end{array}$

$\begin{array}{ll}\text { B Théorème d'Epstein } & 128\end{array}$

C Théorème de Nash-Moser-Hamilton 133

$\begin{array}{ll}\text { Bibliographie } & 139\end{array}$ 



\section{Introduction}

Ce mémoire est consacré à la simplicité des groupes d'homéomorphismes et de difféomorphismes d'une variété compacte, ainsi que de leurs analogues conservatifs, c'est-à-dire préservant une mesure, une forme volume ou une forme symplectique. On rappelle qu'un groupe est dit simple s'il ne contient pas de sous-groupe normal autre que lui-même et le sous-groupe trivial. Puisqu'un sous-groupe normal est invariant par conjugaison, on peut s'attendre à ce qu'un tel sous-groupe dans un groupe de transformations ait un sens dynamique intéressant. Ceci arrive par exemple dans le groupe des difféomorphismes symplectiques d'une variété compacte isotopes à l'identité, dont on verra que le sous-groupe engendré par les commutateurs, qui est toujours normal, est le noyau d'un morphisme de groupes non trivial qui possède une interprétation dynamique. A l'opposé, les résultats de simplicité, autrement dit de non-existence de sous-groupes normaux, s'obtiennent souvent en étudiant la dynamique des éléments du groupe. Donnons deux illustrations de ce fait. La première, abstraite, consiste à remarquer que tous les résultats de simplicité pour les difféomorphismes $C^{\infty}$ utilisent de manière essentielle le théorème de conjugaison locale des difféomorphismes du tore à des translations, qui est un avatar de la théorie K.A.M. La seconde, plus concrète, est une preuve dynamique du fait que le groupe $G$ des homéomorphismes croissants de $\mathbb{R}$ à support compact est simple. Un argument général, sur lequel nous reviendrons, permet de ramener la simplicité à la perfection, c'est-à-dire à montrer que tout élément de $G$ est un produit de commutateurs. Maintenant si l'on prend un élément $f$ de $G$, sa dynamique est alors entièrement décrite par son ensemble de points fixes Fix $(f)$ et par le sens dans lequel $f$ pousse les points sur chaque composante connexe du complémentaire de $F i x(f)$. Cette remarque permet de montrer facilement que $f^{2}$ est conjugué à $f$, i.e. il existe un autre élément $g$ de $G$ tel que $f^{2}=g f g^{-1}$ ce qui peut s'écrire $f=f^{-1} g f g^{-1}=\left[f^{-1}, g\right]$ et donc $f$ est un commutateur.

Dans ce texte, on décide d'énoncer tous les résultats lorsque la variété en question est une surface, bien qu'ils soient valables, mais avec parfois des preuves différentes, en dimension plus grande. La raison de ce choix est qu'en dimension deux, tout est plus "visuel" mais aussi parce que c'est dans ce contexte que certains problèmes de simplicité subsistent pour les 
homéomorphismes conservatifs. On désignera donc par $M$, sauf mention contraire, une surface compacte orientable. En pratique, ce sera la sphère $\mathbb{S}^{2}$, le tore $\mathbb{T}^{2}$ et les surfaces de genre supérieur $\Sigma_{g}$ si $M$ est fermée ou le disque $\mathbb{D}^{2}$, l'anneau $\mathbb{A}$ et les surfaces hyperboliques à bord si $M$ est non fermée. Expliquons maintenant les hypothèses que l'on sera amené à faire sur les groupes que l'on va étudier. Pour obtenir des résultats de simplicité, il est évident qu'il faut commencer par se limiter à la composante connexe de l'identité, qui se trouve être exactement l'ensemble des transformations isotopes à l'identité. Dans le cas à bord, on demandera également aux transformations de fixer un voisinage du bord. Enfin, on rencontrera naturellement des surfaces non compactes, et pour les mêmes raisons, il faudra se restreindre aux transformations à support compact.

Les groupes non conservatifs sont en général simples. Mentionnons deux arguments qui seront récurrents dans les preuves. Le premier est une propriété de fragmentation, c'est-à-dire étant donné un recouvrement ouvert de notre surface, la possibilité de décomposer tout élément du groupe en un produit d'éléments à support dans ces ouverts. Le second est une propriété de transitivité de l'action du groupe, sur des points ou sur des disques. Une utilisation typique de ces arguments est donnée par un théorème très utile d'Epstein (1970) qui nous garantit que si un groupe possède essentiellement ces deux propriétés, il suffit alors de montrer que ce groupe est parfait pour en déduire sa simplicité. Dans le premier chapitre, on étudie les groupes d'homéomorphismes de surface. Le premier résultat obtenu est un théorème non publié de Ulam et Von Neumann (1947) qui affirme que le groupe des homéomorphismes de la sphère préservant l'orientation est simple (ce théorème est également cité dans le "Scottish Book" dans les années 30 où il est attribué à Schreier et Ulam). Mais il faut attendre un théorème général d'Anderson (1958) ainsi que les travaux de Fisher (1960) pour obtenir la simplicité du groupe des homéomorphismes isotopes à l'identité d'une surface $M$ quelconque. C'est alors que Smale posa la question de ce qu'il en était pour les difféomorphismes, et ce sera le thème du second chapitre. Dans le cas particulier des difféomorphismes $C^{\infty}$ du tore isotopes à l'identité, Herman (1973) parvint à prouver la simplicité de ce groupe grâce à un théorème d'inversion locale dans des espaces de Fréchet dû à Sergeraert (1972) et à l'utilisation du théorème d'Epstein. Par des arguments issus de la théorie des espaces classifiants de feuilletages, Thurston (1974, non publié) arriva à en déduire la simplicité dans le cas général. Pour les difféomorphismes de classe $C^{r}$ avec $r$ fini, le théorème d'Herman tombe en défaut mais grâce à des techniques très astucieuses, Mather (1974-1975) réussit à obtenir directement un résultat de simplicité sous la condition que $r$ soit différent de $n+1, n$ étant la dimension de $M$. Le cas où $r=n+1$ reste l'un des grands problèmes ouverts. En revanche, Herman (1975) montre par un procédé d'approximation que son théorème reste valable pour les difféomorphismes analytiques réels. Cependant il semble bien difficile d'étendre ce résultat à d'autres variétés, le souci majeur étant 
l'impossibilité de fragmenter les difféomorphismes analytiques.

Pour les groupes conservatifs, la situation est complètement différente. En effet, sous la condition que le premier groupe d'homologie de la surface soit non trivial, il existe une notion de vecteur de rotation, que l'on peut considérer comme une généralisation du nombre de rotation pour les transformations du cercle, qui donne naissance à un sous-groupe normal propre. La première construction de ce vecteur de rotation remonte probablement à Schwartzman (1957). Dans un troisième chapitre, on étudiera le groupe des difféomorphismes conservatifs, c'est-à-dire préservant un volume, ou ce qui revient au même ici, une forme symplectique. On donnera plusieurs interprétations de cet invariant, en particulier celle du morphisme de flux de Calabi (1970). De ce point de vue, il apparaît que le noyau de ce morphisme coïncide avec les difféomorphismes qui sont associés aux solutions des équations différentielles (non autonomes) de Hamilton, et que l'on appelle groupe des difféomorphismes hamiltoniens. La question naturelle qui se pose est alors la simplicité de ce groupe. Il faut faire ici une distinction selon que $M$ est fermée ou non fermée. Dans le premier cas, le groupe est simple d'après un théorème de Banyaga (1978). On verra que la preuve de ce résultat consiste à adapter les idées de Herman et Thurston. Dans le second cas, on peut définir sur ce groupe un second invariant non trivial, toujours d'après Calabi, mais dont la signification géométrique est, en général, moins évidente. Le théorème de Banyaga s'applique encore à ce noyau et on en déduit qu'il est simple. Enfin, dans le dernier chapitre, on se tournera vers le groupe des homéomorphismes préservant une mesure de Lebesgue. La construction générale du vecteur de rotation dans ce contexte est due à Fathi (1980) mais en revanche la question de la simplicité du noyau demeure ouverte. Cela inclut le cas du groupe des homéomorphismes de la sphère isotopes à l'identité et préservant l'aire ainsi que celui du groupe des homéomorphismes du disque préservant l'aire et fixant un voisinage du bord. Deux approches ont alors été proposées. La première, que l'on doit indépendamment à Fathi (1980) et à Gambaudo et Ghys (1997), consiste à obtenir une interprétation plus visuelle du second invariant de Calabi sur le disque. Malheureusement, cela ne permet pas de l'étendre aux homéomorphismes. On peut néanmoins définir un sousgroupe normal d'après Ghys mais on n'est pas en mesure de décider si c'est un sous-groupe propre. La seconde, que l'on doit à Müller et Oh (2007) et qui est motivée par des questions de dynamique symplectique, consiste à définir un sous-groupe normal d' "homéomorphismes hamiltoniens" qui serait un candidat idéal pour montrer que ces groupes ne sont pas simples. Mais le verdict est le même qu'avant puisque la question de sa propreté reste hors de portée pour le moment. 



\section{Chapitre 1}

\section{Homéomorphismes de surfaces}

Soit $M$ une surface compacte orientable. On désigne par Homeo $(M)$ le groupe des homéomorphismes de $M$, et dans le cas à bord, Homeo $(M, \partial M)$ le groupe des homéomorphismes qui sont l'identité sur un voisinage du bord. On dit qu'un homéomorphisme $h$ est isotope à l'identité si on peut le relier à l'identité par un chemin continu d'homéomorphismes (pour la topologie de la convergence uniforme). Ces homéomorphismes forment un sous-groupe, que l'on note $\operatorname{Homeo}_{0}(M)$, et qui de plus est normal dans Homeo $(M)$. On admet que ce sous-groupe coïncide avec la composante connexe de l'identité de $\operatorname{Homeo}(M)$ (ce dernier point est une conséquence de la contractibilité locale de $\operatorname{Homeo}_{0}(M)$, voir [HD58]). On définit de même le sous-groupe $\operatorname{Homeo}_{0}(M, \partial M)$, avec la condition que toute l'isotopie soit l'identité sur un voisinage du bord.

Le but de ce chapitre est de prouver la simplicité des groupes $\mathrm{Homeo}_{0}(M)$ et $\mathrm{Homeo}_{0}(M, \partial M)$. Les méthodes étant les mêmes dans les deux cas, on ne décrit que le cas sans bord, autrement dit on suppose dorénavant que notre surface $M$ est fermée. Le plan de la preuve est le suivant : on va dans un premier temps caractériser le plus petit sous-groupe normal non trivial de $\operatorname{Homeo}(M)$, que l'on baptise $\operatorname{Homeo}^{0}(M)$, et montrer sa simplicité puis dans un second temps, on va prouver que ce sous-groupe coïncide avec $\mathrm{Homeo}_{0}(\mathrm{M})$.

\subsection{Simplicité du groupe $\operatorname{Homeo}^{0}(M)$}

On se donne une surface fermée $M$. Le disque unité plan fermé sera noté $\mathbb{D}^{2}$. Rappelons qu'un disque fermé (resp. ouvert) dans $M$ est une partie homéomorphe à $\mathbb{D}^{2}$ (resp. à l'intérieur de $\mathbb{D}^{2}$ ). On définit le support d'un 
homéomorphisme $g$ par

$$
\operatorname{supp}(g)=\overline{\{x \in M \mid g x \neq x\}} .
$$

C'est le plus grand fermé sur lequel $g$ agit effectivement. Notons que la propriété d'être à support dans un disque est invariante par conjugaison, puisque pour tout couple d'homéomorphisme $g$ et $h$, le support de $h g h^{-1}$ est l'image par $h$ du support de $g$, i.e. supp $\left(h g h^{-1}\right)=h(\operatorname{supp}(g))$. On en déduit que l'ensemble des homéomorphismes à support dans un disque engendre un sous-groupe normal de $\operatorname{Homeo}(M)$.

Dans tout le chapitre, on fera l'hypothèse que nos disques fermés sont plats au sens suivant : ce sont des images de disques euclidiens fermés inclus dans l'intérieur de $\mathbb{D}^{2}$ par un plongement $\varphi: \mathbb{D}^{2} \hookrightarrow M$. Notons Homeo ${ }^{0}(M)$ le sous-groupe engendré par les homéomorphismes à support dans de tels disques : ce sera le plus petit sous-groupe normal non trivial de Homeo $(M)$, dans le sens où il est contenu dans tout sous-groupe normal non trivial de Homeo $(M)$, et ce groupe sera simple.

Remarque 1.1.1. Le théorème de Schoenflies (annexe A) nous assure que tous les disques fermés en dimension deux vérifient cette condition de platitude.

On aura besoin du lemme suivant, qui énonce une propriété de transitivité (faible) des disques fermés de $M$ sous l'action de $\operatorname{Homeo}^{0}(M)$.

Lemme 1.1.2. Si $F$ et $G$ sont deux disques fermés, alors il existe un élément $f$ dans $\operatorname{Homeo}^{0}(M)$ tel que $f(F)$ soit inclus dans $G$.

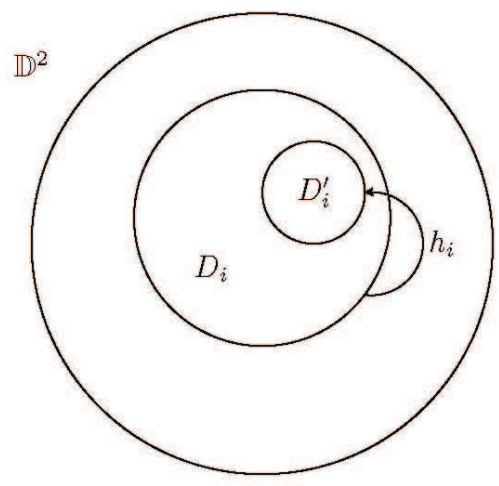

FIG. $1.1-$ Choix des disques $D_{i}^{\prime} \subseteq D_{i}$ et $h_{i}\left(D_{i}\right)=D_{i}^{\prime}$ 
Démonstration. $M$ étant connexe, on peut trouver une suite de disques ouverts $V_{1}, \ldots, V_{k}$ telle que $V_{1}$ soit l'intérieur de $F, V_{i}$ intersecte son voisin $V_{i+1}$ pour $1 \leq i \leq k-1$ et $V_{k}$ est inclus dans l'intérieur de $G$. Pour un indice $i$ dans $\{1, \ldots, k-1\}$, on va construire un homéomorphisme à support dans un disque qui envoie $V_{i}$ dans $V_{i+1}$. On écrit $\overline{V_{i}}=\varphi_{i}\left(D_{i}\right)$, où $D_{i}$ est un disque euclidien fermé inclus dans l'intérieur de $\mathbb{D}^{2}$ et $\varphi_{i}$ un plongement de $\mathbb{D}^{2}$ dans $M$. Puisque l'intérieur de $\overline{V_{i}} \cap \overline{V_{i+1}}$ est non vide, on choisit un petit disque euclidien fermé $D_{i}^{\prime}$ inclus dans $D_{i}$ tel que $\varphi_{i}\left(D_{i}^{\prime}\right)$ soit contenu dans l'intérieur $\overline{V_{i}} \cap \overline{V_{i+1}}$. Soit $h_{i}$ un homéomorphisme de $\mathbb{D}^{2}$, qui est l'identité sur le bord et qui envoie $D_{i}$ sur $D_{i}^{\prime}$ (voir la figure 1.1). L'avantage que l'on a à utiliser des disques euclidiens est que l'on peut construire explicitement un tel homéomorphisme. Soit alors $f_{i}$ l'homéomorphisme défini $\operatorname{par} \varphi_{i} h_{i} \varphi_{i}^{-1}$ sur $\varphi_{i}\left(\mathbb{D}^{2}\right)$ et qui est l'identité ailleurs. Il est clair que $f_{i}$ appartient à $\operatorname{Homeo}^{0}(M)$ et envoie $\overline{V_{i}}$ dans $\overline{V_{i+1}}$. Ainsi $f=f_{k-1} \ldots f_{1}$ est l'homéomorphisme voulu (voir la figure 1.2).

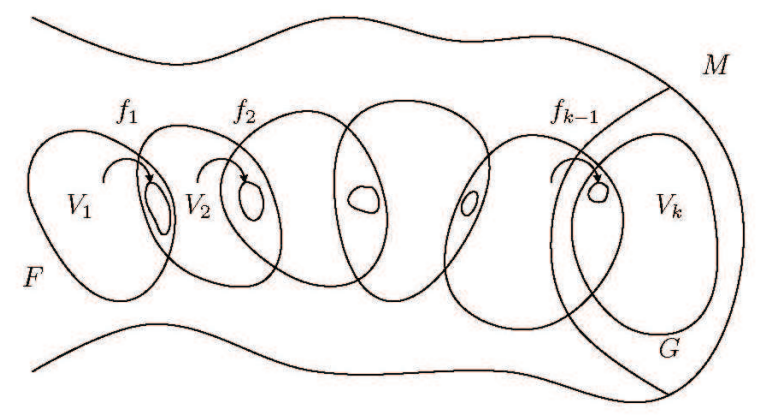

FIG. 1.2 - Construction de $f$ qui envoie $F$ dans $G$

La preuve du lemme précédent est clairement valide en toute dimension. Pour une surface $M$, on peut donner une preuve plus rapide en utilisant le théorème de Schoenflies à support compact (on renvoie à l'annexe A pour un énoncé).

En effet, montrons que l'action de $\operatorname{Homeo}^{0}(M)$ est transitive sur les disques fermés. Soient $D$ et $D^{\prime}$ deux disques fermés de $M$, il faut donc trouver un élément $f$ appartenant à $\operatorname{Homeo}^{0}(M)$ tel que $f(D)=D^{\prime}$. En prenant des points $x$ dans $D$ et $x^{\prime}$ dans $D^{\prime}$, on peut trouver un disque $U$ contenant $x$ et $x^{\prime}$ (par exemple, en prenant un chemin entre $x$ et $x^{\prime}$ et en l'épaississant). En utilisant une première fois le théorème de Schoenflies à support compact (ou une construction explicite), on peut envoyer nos 
disques $D$ et $D^{\prime}$ sur des disques autour de $x$ et $x^{\prime}$, suffisamment petits pour qu'ils soient inclus dans $U$. On se ramène ainsi au cas où $D$ et $D^{\prime}$ sont inclus dans un même disque ouvert. Il nous reste alors à construire un homéomorphisme du plan, à support compact, et qui envoie un disque fermé (non euclidien) sur un autre : c'est exactement ce que fait le théorème de Schoenflies à support compact.

Le résultat principal de cette section est le suivant. On en déduira facilement les propriétés du groupe $\operatorname{Homeo}^{0}(M)$.

Théorème 1.1.3 (Anderson). Soit h un homéomorphisme de $M$ différent de l'identité. Pour tout $f$ appartenant à $\operatorname{Homeo}^{0}(M)$, $f$ est un produit de conjugués de $h$ et $h^{-1}$ par des éléments de $\operatorname{Homeo}^{0}(M)$.

Remarque 1.1.4. Dans la preuve originale de Anderson ([And58]), on trouve que $f$ est un produit d'au plus quatre conjuguées. La présentation suivante est due à Frédéric Le Roux.

Démonstration. Notons $N(h)$ le sous-groupe normal engendré par $h$ dans $\operatorname{Homeo}^{0}(M)$, il faut donc montrer que $N(h)$ contient Homeo $(M)$. L'idée est de prouver que $N(h)$ contient des homéomorphismes de plus en plus généraux.

On commence par montrer que $N(h)$ contient un homéomorphisme $g$ particulier. Puisque $h$ est différent de l'identité, on choisit un point $x$ dans $M$ non fixé par $h$ et un petit disque $D$ centré en $x$ et disjoint de $h(D)$. Écrivons $D=\varphi(D(0,2))$ où $D(0,2)$ est le disque euclidien du plan centré en 0 et de rayon 2 et $\varphi$ un plongement tel que $\varphi(0)=x$. Pour $n \geq 0$, définissons une suite de disques emboîtés $D_{n}=\varphi\left(D\left(0,2^{-n}\right)\right)$. Il est facile de construire explicitement un homéomorphisme $\psi$ à support dans $D$ tel que $\psi\left(D_{n}\right)=D_{n+1}$ pour $n \in \mathbb{N}$ (on fait la construction dans le plan et on la transporte par $\varphi$ ). Alors l'homéomorphisme

$$
g=[\psi, h] \in N(h)
$$

vérifie $g\left(D_{n}\right)=D_{n+1}$ pour $n \geq 0$ et $g$ est à support dans $D \cup h(D)(g$ est le produit des homéomorphismes $\psi$ et $h \psi^{-1} h^{-1}$ qui sont à support disjoints).

Notons $A_{n}=D_{n} \backslash D_{n+1}$, pour $n \geq 0$. On montre maintenant que $N(h)$ contient tous les homéomorphismes à support dans l'anneau $A_{1}$. En effet, si $\phi$ est un tel homéomorphisme, alors $\phi$ conjugue $g$ à $\phi g$ sur $A_{0}$, i.e.

$$
g=\phi^{-1}(\phi g) \phi \operatorname{sur} A_{0}
$$

ainsi que sur les itérés négatifs $g^{n}\left(A_{0}\right), n<0$, puisque $\phi$ est alors l'identité. Maintenant, puisque $g^{n}\left(A_{0}\right)$ converge vers $x$ lorsque $n$ tend vers $+\infty$, il y a une unique manière d'étendre la conjugaison sur

$$
D_{0}=\left(\cup_{n \in \mathbb{N}} A_{n}\right) \cup\{x\} .
$$


Ainsi $g$ et $\phi g$ sont conjugués, donc $\phi g \in N(g) \subseteq N(h)$ puis $\phi \in N(h)$.

Enfin, si on se donne un homéomorphisme $f$ à support dans un disque fermé quelconque $F$, par le lemme 1.1.2 on le conjugue à un homéomorphisme dont le support est dans l'anneau $A_{1}$, avec une conjugante appartenant à $\operatorname{Homeo}^{0}(M)$. On obtient bien que $N(h)$ contient $\operatorname{Homeo}^{0}(M)$.

Le corollaire suivant est alors immédiat.

Corollaire 1.1.5. Le groupe $\operatorname{Homeo}^{0}(M)$ est simple. De plus, c'est le plus petit sous-groupe normal de Homeo $(M)$.

Démonstration. Soit $H$ un sous-groupe normal non trivial de $\operatorname{Homeo}^{0}(M)$, et $h$ un élément appartenant à $H$ différent de l'identité. Il suffit de prendre un élément arbitraire $f \in \operatorname{Homeo}^{0}(M)$ et de l'écrire comme un produit de conjugués de $h$ et $h^{-1}$ par des éléments de $\operatorname{Homeo}^{0}(M)$ pour obtenir que le groupe $\operatorname{Homeo}^{0}(M)$ est inclus dans $H$. Ainsi $\operatorname{Homeo}^{0}(M)$ est un groupe simple. Pour obtenir la seconde partie de l'énoncé, il suffit d'appliquer le raisonnement précédent avec cette fois-ci $H$ un sous-groupe normal non trivial de $\operatorname{Homeo}(M)$.

Exemple 1.1.6 (Le disque $\left.\mathbb{D}^{2}\right)$. Le corollaire précédent nous donne déjà la simplicité du groupe

$$
\text { Homeo }\left(\mathbb{D}^{2}, \partial \mathbb{D}^{2}\right)=\text { Homeo }^{0}\left(\mathbb{D}^{2}, \partial \mathbb{D}^{2}\right) .
$$

On en déduit facilement la simplicité du groupe $\mathrm{Homeo}_{0}\left(\mathbb{D}^{2}, \partial \mathbb{D}^{2}\right)$. En effet, il suffit de prouver que Homeo $\left(\mathbb{D}^{2}, \partial \mathbb{D}^{2}\right)$ est connexe par arcs, et ceci est une conséquence de l' "astuce d'Alexander" : si $\varphi \in H$ omeo $\left(\mathbb{D}^{2}, \partial \mathbb{D}^{2}\right)$, alors

$$
\varphi_{t}(x)= \begin{cases}t \varphi\left(\frac{x}{t}\right) & \text { si } d(0, x) \leq t \\ x & \text { si } d(0, x) \geq t\end{cases}
$$

est un chemin continu dans $\operatorname{Homeo}\left(\mathbb{D}^{2}, \partial \mathbb{D}^{2}\right)$ reliant $\varphi_{0}=1$ à $\varphi_{1}=\varphi$.

Puisque $\mathrm{Homeo}_{0}(M)$ est aussi un sous-groupe normal de $\operatorname{Homeo}(M)$, on a l'inclusion

$$
\operatorname{Homeo}^{0}(M) \subseteq \text { Homeo }_{0}(M)
$$

(qui peut également s'obtenir directement par l'astuce d'Alexander). Dans la section suivante, on va montrer que c'est en fait une égalité.

\subsection{Simplicité du groupe $\mathrm{Homeo}_{0}(M)$}

Passons maintenant à l'étude du groupe des homéomorphismes de $M$ isotopes à l'identité, et démontrons sa simplicité. D'après la section précédente, il suffit de montrer que tout élément de $\operatorname{Homeo}_{0}(M)$ s'écrit comme produit d'homéomorphismes à support dans des disques. Commençons par un exemple simple. 
Exemple 1.2.1 (La sphère $\mathbb{S}^{2}$ ). On peut décomposer tout homéomorphisme de la sphère préservant l'orientation (qui est équivalent ici à être isotope à l'identité) en un produit de deux homéomorphismes à support dans des disques. L'argument utilise (encore une fois) le théorème de Schoenflies à support compact. Prenons $f \in \mathrm{Homeo}_{0}\left(\mathbb{S}^{2}\right)$ différent de l'identité, et $x$ dans $\mathbb{S}^{2}$ un point qui ne soit pas fixe par $f$. On se donne un disque fermé $D$ de $\mathbb{S}^{2}$ contenant $x$ et $f(x)$, et un petit disque ouvert $D^{\prime}$ contenant $x$, disjoint de son image et tel que l'union $D^{\prime} \cup f\left(D^{\prime}\right)$ soit dans $D$ (voir la figure 1.3). Par le théorème de Schoenflies à support compact, la restriction de $f \grave{a}$ $D^{\prime}$ s'étend en un homéomorphisme $g$ à support dans $D$ qui envoie $D^{\prime}$ sur $f\left(D^{\prime}\right)$. On écrit alors $f=g\left(g^{-1} f\right)$, avec $g$ à support dans $D$ et $g^{-1} f \grave{a}$ support dans $\mathbb{S}^{2} \backslash D^{\prime}$.

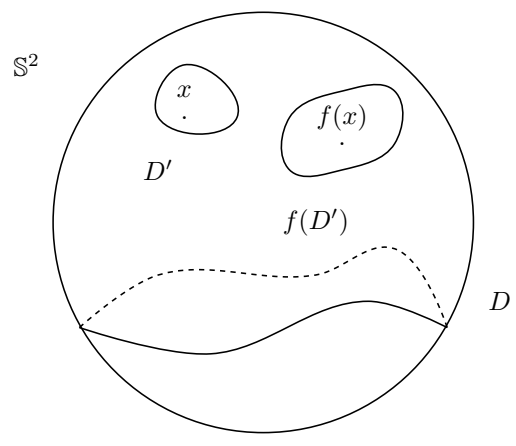

FIG. 1.3 - Simplicité de $\mathrm{Homeo}_{0}\left(\mathbb{S}^{2}\right)$

Dans le cas général, on va en fait obtenir une telle décomposition pour des disques arbitrairement petits, ce qui va nous donner la propriété de fragmentation. Commençons par définir cette notion de manière formelle, car on l'utilisera à de nombreuses reprises dans ce texte.

Définition 1.2.2. On dit qu'un sous-groupe $G$ de Homeo $(M)$ a la propriété de fragmentation si pour tout recouvrement ouvert $\mathcal{U}=\left(U_{i}\right)_{i \in I}$ de $M$ et pour tout élément $g$ appartenant à $G$, il existe des éléments $g_{1}, \ldots g_{n} \in G$ tels que

$$
g=g_{1} \ldots g_{n}
$$

et pour $1 \leq i \leq n, g_{i}$ est à support dans un des éléments de $\mathcal{U}$.

On munit Homeo $(M)$ de la topologie de la convergence uniforme, i.e. de la topologie définie par la distance (complète)

$$
d_{C^{0}}(f, g)=\sup _{x \in M} d(f(x), g(x))+\sup _{x \in M} d\left(f^{-1}(x), g^{-1}(x)\right)
$$


où $d$ est une distance quelconque compatible avec la topologie sur $M$. Avec cette topologie, Homeo $(M)$ est un groupe topologique.

Le résultat suivant est dû à Fisher ([Fis60]). Il repose essentiellement sur le théorème de Schoenflies ainsi que ses variantes, que l'on expose brièvement dans l'annexe $\mathrm{A}$.

Théorème 1.2.3 (Fisher). Si $M$ est une surface compacte, alors le groupe $\mathrm{Homeo}_{0}(\mathrm{M})$ possède la propriété de fragmentation.

Démonstration. Soit $\mathcal{U}=\left\{U_{1}, \ldots, U_{m}\right\}$ un recouvrement ouvert de $M$. Par connexité du groupe Homeo $_{0}(M)$, tout élément s'écrit comme un produit d'éléments arbitrairement proches de l'identité. Il est alors suffisant de fragmenter un élément $f$ voisin de l'identité.

La surface $M$ étant triangulable, on peut supposer que c'est la réalisation géométrique d'un 2-complexe simplicial fini $T$. Quitte à subdiviser $T$, on peut supposer que chaque triangle est contenu dans l'un des ouverts de $\mathcal{U}$ et qu'il a un diamètre petit devant $\delta$, où $\delta$ est le minimum des diamètres des éléments de $\mathcal{U}$.

La preuve se décompose alors en deux étapes détaillées ci-dessous. On commence par rectifier $f$ par un élément $u \in \operatorname{Homeo}^{0}(M)$ au voisinage des sommets pour obtenir $g=u^{-1} f$ qui soit l'identité au voisinage des sommets. Puis on modifie de même $g$ par un élément $v \in \operatorname{Homeo}^{0}(M)$ au voisinage des arêtes. On se retrouve alors avec $h=v^{-1} g$ qui est l'identité au voisinage du bord de chaque triangle et qui par conséquent les préserve : il est alors évident que $h \in \operatorname{Homeo}^{0}(M)$ et donc que $f$ appartient à $\operatorname{Homeo}^{0}(M)$. De plus, on obtient une écriture

$$
f=u v h=u_{1} \ldots u_{n} v_{1} \ldots v_{t} h_{1} \ldots h_{t}
$$

avec chaque homéomorphisme à support dans l'un des disques de $\mathcal{U}$ (si l'on a choisi une triangulation suffisamment fine).

(a) Rectification de $f$ le long du 0-squelette.

On se donne un réel positif $r$ (que l'on choisira dans la seconde étape) et on cherche $s>0$ tel que si $d_{C^{0}}(f, 1)<s$, il existe un homéomorphisme $g=$ $u^{-1} f$ avec $u \in$ Homeo $^{0}(M)$, qui soit l'identité au voisinage des sommets et tel que $d_{C^{0}}(g, 1)<r / 2$.

Précisons quelques notations. On considère $T^{1}$ et $T^{2}$ les première et seconde subdivisions barycentriques de $T$ (voir la figure 1.4). Notons $a_{1}, \ldots, a_{n}$ les sommets de $T$ et, pour $1 \leq i \leq n, D_{i}^{\prime}$ et $D_{i}^{\prime \prime}$ les étoiles fermées de $a_{i}$ dans $T^{1}$ et $T^{2}$ respectivement (rappelons que l'étoile fermé d'un sommet est la réunion de tous les simplexes contenant ce sommet, voir la figure 1.5). On peut voir les $D_{i}^{\prime \prime}$ et $D_{i}^{\prime}$ comme des voisinages fermés de $a_{i}$, et on a les propriétés suivantes : les $D_{i}^{\prime}$ forment un recouvrement de $M$ et ils ne s'intersectent que le long de leurs bords, $D_{i}^{\prime \prime}$ est inclus dans l'intérieur de $D_{i}^{\prime}$ et les 


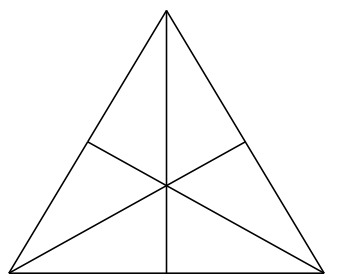

$T^{1}$

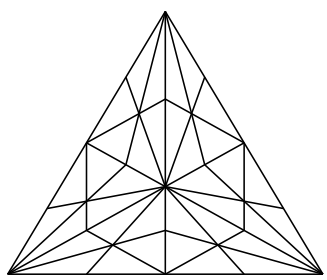

$T^{2}$

FIG. 1.4 - Subdivisions $T^{1}$ et $T^{2}$
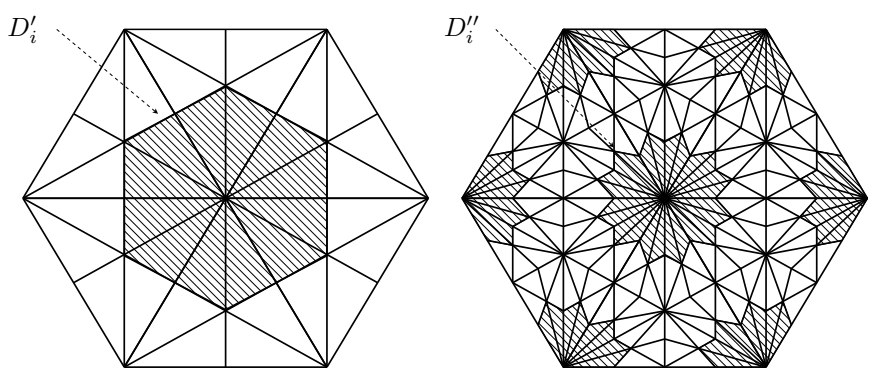

FIG. 1.5 - Étoiles $D_{i}^{\prime}$ et $D_{i}^{\prime \prime}$ 
$D_{i}^{\prime \prime}$ sont deux à deux disjoints. Fixons un indice $1 \leq i \leq n$. On choisit des disques $D_{2} \subseteq D_{1}$ dans $\mathbb{R}^{2}$ centrés à l'origine et des homéomorphismes $\varphi_{i}$ de $D_{1}$ sur $D_{i}^{\prime}$ envoyant $D_{2}$ dans $D_{i}^{\prime \prime}$. Voici plusieurs remarques (rappelons que l'on s'est donné un réel $r$ positif).

(i) Par continuité uniforme des $\varphi_{i}$, il existe $r^{\prime}>0$ tel que

$$
d(x, y)<r^{\prime} \Longrightarrow d\left(\varphi_{i}(x), \varphi_{i}(y)\right)<r / 4 \text {. }
$$

(ii) La version "à paramètres" du théorème de Schoenflies donne $s^{\prime}>0$ tel que si $f: D_{2} \hookrightarrow D_{1}$ est un plongement avec $d_{C^{0}}\left(f, 1_{D_{2}}\right)<s^{\prime}$, alors $f$ admet une extension $\bar{f}: D_{1} \rightarrow D_{1}$ avec $\bar{f}_{\mid \partial D_{1}}=1$ et $d_{C^{0}}\left(\bar{f}, 1_{D_{1}}\right)<r^{\prime}$.

(iii) Par continuité uniforme des $\varphi_{i}^{-1}$, il existe $\left.s \in\right] 0, r / 4[$ tel que

$$
d\left(x^{\prime}, y^{\prime}\right)<s \Longrightarrow d\left(\varphi_{i}^{-1}\left(x^{\prime}\right), \varphi_{i}^{-1}\left(y^{\prime}\right)\right)<s^{\prime} .
$$

Prenons donc $f$ un élément de $\operatorname{Homeo}(M)$ vérifiant $d_{C^{0}}(f, 1)<s$. Pour $x$ appartenant à $D_{2}$, on a

$$
d\left(f \varphi_{i}(x), \varphi_{i}(x)\right)<s
$$

donc par $(i i i)$,

$$
d\left(\varphi_{i}^{-1} f \varphi_{i \mid D_{2}}, 1_{D_{2}}\right)<s^{\prime}
$$

On applique alors $(i i)$ à $\varphi_{i}^{-1} f \varphi_{i \mid D_{2}}$ pour obtenir une extension à $D_{1}$ qu'on note $f_{i}$. Ainsi $f_{i \mid \partial D_{1}}=1$ et $d_{C^{0}}\left(f_{i}, 1_{D_{1}}\right)<r^{\prime}$. Pour $x^{\prime}$ appartenant à $\varphi_{i}\left(D_{1}\right)=D_{i}^{\prime}$, on a

$$
d\left(f_{i} \varphi_{i}^{-1}\left(x^{\prime}\right), \varphi_{i}^{-1}\left(x^{\prime}\right)\right)<r^{\prime}
$$

et $(i)$ nous donne

$$
d\left(\varphi_{i} f_{i} \varphi_{i}^{-1}\left(x^{\prime}\right), x^{\prime}\right)<r / 4 .
$$

Définissons $u_{i}$ par $\varphi_{i} f_{i} \varphi_{i}^{-1}$ sur $D_{i}^{\prime}$ et l'identité ailleurs (ce qui est cohérent car les $\varphi_{i} f_{i} \varphi_{i}^{-1}$ sont l'identité sur $\left.\partial D_{i}^{\prime}\right)$. On a ainsi $d_{C^{0}}\left(u_{i}, 1\right)<r / 4, u_{i \mid D_{i}^{\prime \prime}}=$ $f_{\mid D_{i}^{\prime \prime}}$ et $u_{i} \in \operatorname{Homeo}^{0}(M)$. Posons enfin $u=u_{1} \ldots u_{n} \in \operatorname{Homeo}^{0}(M)^{2}$ et $g=u^{-1} f$. Alors $u$ est un élément de $\operatorname{Homeo}^{0}(M)$ qui va modifier $f$ en le $g$ voulu. Pour $x \in M$, ou bien $x$ est dans l'intérieur d'un unique $D_{i}^{\prime}$ et dans ce cas $u(x)=u_{i}(x)$, ou bien $x$ est sur le bord d'un des $D_{i}^{\prime}$ et alors $u(x)=x$. On en déduit que $d_{C^{0}}(u, 1)<r / 4$. L'inégalité triangulaire donne aussitôt

$d_{C^{0}}(g, 1) \leq d_{C^{0}}\left(u^{-1}, 1\right)+d_{C^{0}}(f, 1)=d_{C^{0}}(u, 1)+d_{C^{0}}(f, 1)<r / 4+s<r / 2$.

Enfin $u_{\mid D_{i}^{\prime \prime}}=u_{i \mid D_{i}^{\prime \prime}}=f_{\mid D_{i}^{\prime \prime}}$ et donc $g$ est l'identité sur $V=\bigcup_{i=1}^{n} D_{i}^{\prime \prime}$. Ceci termine la première étape : il suffit maintenant de vérifier que $g$, qui est l'identité au voisinage du 0 -squelette de $T$, appartient à $\operatorname{Homeo}^{0}(M)$. 
(b) Rectification de g le long du 1-squelette.

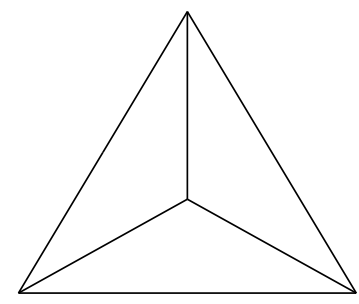

$T^{\prime}$

FIG. 1.6 - Subdivision $T^{\prime}$

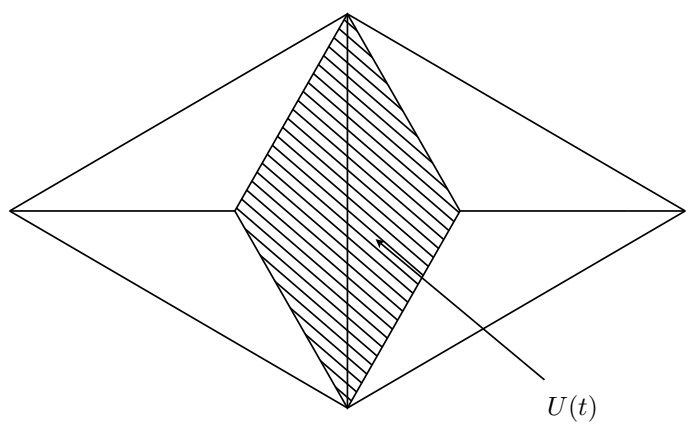

FIG. 1.7 - Ensemble $U(t)$

On introduit maintenant une nouvelle subdivision $T^{\prime}$ de $T$, que l'on obtient en mettant une arête entre le barycentre de chaque triangle de $T$ et ses trois sommets (voir la figure 1.6). On note $T_{1}^{\prime}$ le 1 -squelette de $T^{\prime}$ (c'est-à-dire le complexe simplicial formé des sommets et des arêtes de $T^{\prime}$ ) et $\left|T_{1}^{\prime}\right|$ sa réalisation géométrique. Pour chaque arête $t$ de $T$, notons $U(t)$ l'union des deux triangles de $T^{\prime}$ ayant $t$ comme arête commune (on peut voir les $U(t)$ comme des voisinages des arêtes de $T$, voir la figure 1.7). Pour chaque triangle $S$ de $T^{\prime}$, notons $t_{S}$ son arête dans $T$. Définissons sur $\partial S$ une application $v_{S}$ qui cö̈ncide avec $g$ sur $t_{S}$ et qui est l'identité sur les 2 autres arêtes. Alors $v_{S}$ est continue $\left(\operatorname{car} g_{\mid V}=1\right)$ et $v_{S}$ envoie $\partial S$ dans $U\left(t_{S}\right)$ : en 
effet, on sait que $d_{C^{0}}(g, 1)<r / 2$, il suffit alors de choisir $r$ dans la première étape comme étant la distance minimale entre 2 composantes connexes de $\left|T_{1}^{\prime}\right| \backslash V$. Donc $v_{S}$ est un homéomorphisme de $\partial S$ sur son image, par le théorème de Schoenflies (classique) il s'étend en un homéomorphisme de $S$ sur son image. Ces homéomorphismes se recollent de manière évidente en un homéomorphisme $v$ tel que $v_{\mid \partial U(t)}=1$, car les $U(t)$ forment un recouvrement fini de $T$. Cela permet de définir $v_{t}$ par valant $v$ sur $U(t)$ et l'identité ailleurs. Les $v_{t}$ sont dans $\operatorname{Homeo}^{0}(M)$, donc $v$ appartient à $\operatorname{Homeo}^{0}(M)$. Posons alors $h=v^{-1} g$. Puisque $h_{\mid \partial S}=1$ pour tout triangle $S$ de $T$ et que $h$ envoie $S$ dans lui-même, le raisonnement précédent montre alors que $h$ appartient à $\operatorname{Homeo}^{0}(M)$. On achève ainsi la seconde étape : on a modifié $g$ en un $h$ qui est l'identité sur le 1 -squelette de $T$ et qui par conséquent est dans $\operatorname{Homeo}^{0}(M)$. Pour conclure, $f=u g=u v h \in \operatorname{Homeo}^{0}(M)$, ce qui prouve le théorème.

Comme corollaire des théorèmes 1.1 .3 et 1.2 .3 , on a immédiatement le résultat de ce chapitre.

Théorème 1.2.4. Le groupe des homéomorphismes d'une surface compacte isotopes à l'identité est simple.

Pour conclure, expliquons comment généraliser les théorèmes précédents en dimension supérieure.

Les résultats sur le groupe $\operatorname{Homeo}^{0}(M)$ sont valables en dimension plus grande, avec les mêmes preuves. On peut également utiliser les arguments liés au théorème de Schoenflies (version classique et version à support compact) même si dans ce second cas, les résultats que l'on utilise sont notoirement plus difficiles à prouver (voir l'annexe A pour des remarques sur le théorème de Schoenflies en dimension plus grande que deux).

En ce qui concerne la simplicité de $\mathrm{Homeo}_{0}(M)$, on a utilisé de manière essentielle une triangulation de la surface ce qui ne permet pas d'étendre la preuve à toutes les dimensions (cela marche encore pour $\operatorname{dim}(M)=$ 3 , voir [Fis60], mais rappelons que les variétés topologiques de dimension plus grande n'admettent pas nécessairement de triangulation). Néanmoins, la propriété de fragmentation est encore valide, mais cela est bien plus compliqué (voir [EK71]).

Enfin, en termes homologiques, on obtient ici que le premier groupe d'homologie de $\operatorname{Homeo}_{0}(M)$ est trivial. En utilisant les techniques d'Anderson, Mather ([Mat74b]) a aussi démontré que l'homologie du groupe $\mathrm{Homeo}_{0}(\mathrm{M})$ est triviale. 


\section{Chapitre 2}

\section{Difféomorphismes de surfaces}

Dans ce chapitre, on va s'intéresser aux groupes de difféomorphismes Dif $f_{0}^{r}(M)$ et Dif $f_{0}^{r}(M, \partial M)$ d'une surface compacte $M$, pour $1 \leq r \leq \infty$. Les isotopies seront de classe $C^{r}$, et dans le cas à bord, on demande qu'elles soient à support dans l'intérieur de $M$. Comme précédemment, on énoncera les résultats seulement lorsque $M$ est fermée (le cas à bord s'obtient par de légères modifications).

Pour $r=\infty$, on va montrer que ce groupe est simple, mais les arguments seront plus délicats que ceux du chapitre précédent. La preuve se découpe essentiellement en deux parties : une partie analytique que l'on doit principalement à Herman où l'on démontre le résultat pour $M=\mathbb{T}^{2}$ et une partie "géométrique" due à Thurston et Mather qui permet d'obtenir le résultat général à partir du cas particulier du tore. Pour $r$ fini, on expliquera un résultat de Mather sur la simplicité de $D$ if $f_{0}^{r}(M)$ sous la restriction que $r$ soit différent de 3 . Le cas où $r=3$ est encore non résolu.

Notons enfin que tous les résultats et les méthodes présentés dans ce chapitre n'ont absolument rien de spécifique à la dimension deux, et qu'ils s'étendent à toutes les dimensions.

\subsection{Simplicité du groupe $\operatorname{Dif} f_{0}^{\infty}\left(\mathbb{T}^{2}\right)$}

On rappelle qu'un groupe $G$ est parfait si tout élément s'écrit comme un produit de commutateurs. Dans cette section, on souhaite démontrer le théorème suivant ([Her73]).

Théorème 2.1.1 (Herman). Le groupe Dif $f_{0}^{\infty}\left(\mathbb{T}^{2}\right)$ est parfait.

Par un argument général d'Epstein (voir l'annexe B pour plus de détails), on obtient le corollaire suivant. 
Corollaire 2.1.2. Le groupe Dif $f_{0}^{\infty}\left(\mathbb{T}^{2}\right)$ est simple.

Commençons par quelques préliminaires. Pour $k=\left(k_{1}, k_{2}\right)$ appartenant à $\mathbb{Z}^{2}$, notons $|k|=\left|k_{1}\right|+\left|k_{2}\right|$ et si $\gamma=\left(\gamma_{1}, \gamma_{2}\right)$ est dans $\mathbb{T}^{2}$, on pose

$$
k \cdot \gamma=k_{1} \gamma_{1}+k_{2} \gamma_{2} \in \mathbb{T} \text {. }
$$

On définit une norme sur $\mathbb{T}$ par

$$
\|\mid \alpha\|=d(\tilde{\alpha}, \mathbb{Z})
$$

où $\tilde{\alpha} \in \mathbb{R}$ est un relevé de $\alpha \in \mathbb{T}$ (la définition est clairement indépendante du choix du relevé).

Définition 2.1.3. Un vecteur $\gamma \in \mathbb{T}^{2}$ vérifie une condition diophantienne de type $(c, \tau)$ s'il existe $c>0, \tau \geq 0$ tels que pour tout $k \in \mathbb{Z}^{2} \backslash\{0\}$ on ait

$$
|| k \cdot \gamma|\|| \geq c|k|^{-2-\tau} \text {. }
$$

Remarque 2.1.4. Dans la définition précédente, certains auteurs utilisent $\max \left(\left|k_{1}\right|,\left|k_{2}\right|\right)$ au lieu de $|k|=\left|k_{1}\right|+\left|k_{2}\right|$.

Cette condition arithmétique est une hypothèse classique dans la théorie K.A.M. (Kolmogorov, Arnold et Moser) pour les difféomorphismes, elle nous assure que les vecteurs vérifiant une telle condition diophantienne sont "mal approchés" par les vecteurs dont les coordonnées sont rationnelles et de même dénominateur.

Notons $\mathcal{C D}(c, \tau)$ l'ensemble des vecteurs diophantiens de type $(c, \tau)$ et

$$
\mathcal{C D}(\tau)=\bigcup_{c>0} \mathcal{C D}(c, \tau) \quad ; \quad \mathcal{C D}=\bigcup_{\tau \geq 0} \mathcal{C D}(\tau)
$$

On peut donner des exemples explicites de tels vecteurs. Cependant, pour s'assurer que cette notion n'est pas vide, il est plus agréable de passer par la proposition suivante.

Proposition 2.1.5. L'ensemble $\mathcal{C D}$ est de mesure totale pour la mesure de Haar sur $\mathbb{T}^{2}$. En particulier, il existe des vecteurs diophantiens.

Cela résulte du fait que $\mathcal{C D} \cap[0,1]^{2}$ est de mesure totale pour la mesure de Lebesgue sur le carré. En effet, à $\tau>0$ fixé, on peut majorer la mesure du complémentaire de $\mathcal{C D}(c, \tau)$ dans $[0,1]$ par une constante proportionnelle à $c$, ce qui implique que $[0,1]^{2} \backslash \mathcal{C D}(c, \tau)$ est de mesure arbitrairement petite si $c$ est arbitrairement petit et donc $\mathcal{C D}(\tau)$ est de mesure totale (voir [Lan95] pour plus de détails).

Remarque 2.1.6. Bien que génériques pour la mesure, les vecteurs diophantiens ne le sont pas du tout pour la topologie : on montre facilement qu'ils forment un ensemble maigre au sens de Baire, i.e. qu'ils sont contenus dans une réunion dénombrable de fermés d'intérieurs vides. 
Pour $\gamma$ appartenant à $\mathbb{T}^{2}$, on note $R_{\gamma}$ la translation de vecteur $\gamma$. Les translations forment un sous-groupe de Diff $f_{0}^{\infty}\left(\mathbb{T}^{2}\right)$ qui s'identifie à $\mathbb{T}^{2}$. Dans la suite, Dif $f_{0}^{\infty}\left(\mathbb{T}^{2}\right)$ sera muni de la topologie $C^{\infty}$.

Commençons par expliquer les idées de la preuve du théorème d'Herman. On souhaite décomposer tout élément de Dif $f_{0}^{\infty}\left(\mathbb{T}^{2}\right)$ en un produit de commutateurs. Par des arguments de connexité, il suffit de le faire pour un élément arbitrairement proche de l'identité. Il n'est pas très dur de vérifier qu'une translation du tore $R_{\gamma}$ est un produit de commutateurs : on commence par la décomposer en un produit de deux rotations du cercle (formellement, deux translations du tore dont le vecteur a une coordonnée nulle), puis on utilise le fait que les rotations sont des homographies particulières de la droite projective réelle et que $P S L(2, \mathbb{R})$ est parfait pour aboutir au résultat. Cependant, il est plus dur d'obtenir une telle écriture si l'on perturbe légèrement $R_{\gamma}$. Si tel était le cas, la différence entre $R_{\gamma}$ et sa perturbation nous donnerait un élément arbitrairement proche de l'identité qui s'écrit comme un produit de commutateurs. Par chance, pour un vecteur $\gamma$ diophantien, on peut décrire assez explicitement les perturbations de $R_{\gamma}$.

La preuve du théorème repose donc entièrement sur le résultat K.A.M. suivant, qui affirme que si un difféomorphisme du tore est suffisamment proche d'une translation diophantienne $R_{\gamma}$, alors quitte à le rectifier par une petite translation, il lui est $C^{\infty}$-conjuguée. Un tel difféomorphisme s'écrit donc sous une forme normale

$$
R_{\lambda} \psi^{-1} R_{\gamma} \psi=R_{\lambda+\gamma}\left(R_{\gamma}^{-1} \psi^{-1} R_{\gamma} \psi\right)
$$

pour $\lambda \in \mathbb{T}^{2}$ et $\psi \in \operatorname{Diff} f_{0}^{\infty}\left(\mathbb{T}^{2}\right)$, ce qui montre bien qu'il est un produit de commutateurs. Voici l'énoncé précis, dont la preuve originale est due à Sergeraert et Herman ([Ser72], [Her73]).

Théorème 2.1.7 (Sergeraert-Herman). Soit $R_{\gamma}$ une translation de vecteur diophantien $\gamma$ et soit l'application

$$
\begin{aligned}
& \Phi_{\gamma}: \operatorname{Diff}_{0}^{\infty}\left(\mathbb{T}^{2}\right) \times \mathbb{T}^{2} \longrightarrow \operatorname{Diff} f_{0}^{\infty}\left(\mathbb{T}^{2}\right) \\
& (\psi, \lambda) \quad \longmapsto R_{\lambda} \psi^{-1} R_{\gamma} \psi
\end{aligned}
$$

Alors il existe un voisinage $V$ de $R_{\gamma}$ dans Dif $f_{0}^{\infty}\left(\mathbb{T}^{2}\right)$ et une application lisse

$$
s: V \longrightarrow \operatorname{Diff} f_{0}^{\infty}\left(\mathbb{T}^{2}\right) \times \mathbb{T}^{2}
$$

telle que $s\left(R_{\gamma}\right)=\left(1_{\mathbb{T}^{2}}, 0\right)$ et $\Phi_{\gamma} s=1_{V}$.

Démonstration. On souhaite résoudre une équation non linéaire. Il est beaucoup plus simple, lorsque cela est possible, de résoudre l'équation linéarisée et de conclure par un théorème d'inversion locale. Cependant, en admettant la structure différentiable de l'espace Dif $f_{0}^{\infty}\left(\mathbb{T}^{2}\right)$ (que l'on suppose implicitement dans l'énoncé et qui sera précisée plus loin), l'espace 
linéaire associé n'est pas un espace de Banach ce qui empêche l'utilisation du théorème d'inversion locale classique. Pour remédier à ce problème, on va utiliser un autre théorème d'inversion, le théorème de Nash-MoserHamilton, valable dans une certaine catégorie d'espaces de Fréchet (les bons espaces de Fréchet et les bonnes applications entre bons espaces de Fréchet) mais sous des hypothèses plus restrictives. On renvoie à l'annexe $\mathrm{C}$ pour ces notions.

Remarquons que $\Phi_{\gamma}\left(1_{\mathbb{T}^{2}}, 0\right)=R_{\gamma}$. Pour pouvoir appliquer le théorème de Nash-Moser-Hamilton, on doit donc prouver que la différentielle $d \Phi_{\gamma}$ est inversible en tout point d'un voisinage de $\left(1_{\mathbb{T}^{2}}, 0\right)$ dans Dif $f_{0}^{\infty}\left(\mathbb{T}^{2}\right) \times \mathbb{T}^{2}$, et que son inverse est une bonne application linéaire continue. Selon Herman, on procède en quatre étapes.

(a) On exprime $\Phi_{\gamma}$ dans une carte exponentielle.

Fixons une métrique riemannienne (nécessairement complète) sur $\mathbb{T}^{2}$ et considérons l'application exponentielle associée

$$
\begin{array}{cccc}
\exp : & \mathrm{TT}^{2} & \longrightarrow & \mathbb{T}^{2} \times \mathbb{T}^{2} \\
& (x, v) & \longmapsto & \left(x, \exp _{v}(x)\right)
\end{array}
$$

où $\exp _{v}(x)$ est le temps 1 de l'unique géodésique de conditions initiales $(x, v)$. Il est bien connu que l'exponentielle se restreint en un difféomorphisme d'un voisinage $V$ de la section nulle de $\mathrm{TT}^{2}$ sur un voisinage $W$ de la diagonale de $\mathbb{T}^{2} \times \mathbb{T}^{2}$. Pour un difféomorphisme $f$, posons

$$
W_{f}=\left\{g \in \operatorname{Diff} f_{0}^{\infty}\left(\mathbb{T}^{2}\right) \mid \forall x \in \mathbb{T}^{2},(f(x), g(x)) \in W\right\}
$$

et

$$
V_{f}=\left\{\xi \in \Gamma_{f}^{\infty}\left(\mathbb{T}^{2}\right) \mid \forall x \in \mathbb{T}^{2}, \xi(x) \in V\right\}
$$

où $\Gamma_{f}^{\infty}\left(\mathbb{T}^{2}\right)$ est l'espace vectoriel des champs de vecteurs au-dessus de $f$, i.e. l'espace des sections du fibré induit $f^{*}\left(\mathrm{TT}^{2}\right.$ ) (c'est l'espace qui joue le rôle d'espace tangent au dessus de $f$ ). On peut alors définir un difféomorphisme

$$
\phi_{f}: V_{f} \longrightarrow W_{f}
$$

à l'aide de l'application exponentielle : on obtient ainsi une collection de cartes $\left(W_{f}, \phi_{f}^{-1}\right)$ permettant de munir Diff $f_{0}^{\infty}\left(\mathbb{T}^{2}\right)$ d'une structure lisse modelée sur $\Gamma^{\infty}\left(\mathbb{T}^{2}\right)$ (la vérification que l'on obtient bien un atlas est loin d'être immédiate, voir [Mil84] par exemple).

Sur $\mathbb{R}^{2}$ muni de sa métrique canonique, on a plus simplement

$$
\exp (x, v)=(x, x+v)
$$

donc quitte à relever les difféomorphismes du tore en des difféomorphismes du plan $\mathbb{R}^{2}$ (en relevant une isotopie à l'identité), on peut écrire

$$
\phi_{f}(\xi)(x)=f(x)+\xi(x)
$$




$$
\phi_{f}^{-1}(g)(x)=g(x)-f(x) .
$$

L'application $\Phi_{\gamma}$ lue dans ces cartes s'écrit :

$$
\begin{array}{ccc}
\Gamma^{\infty}\left(\mathbb{T}^{2}\right) \times \mathbb{R}^{2} & \longrightarrow & \Gamma_{R_{\gamma}}^{\infty}\left(\mathbb{T}^{2}\right) \\
(\xi, \lambda) & \longmapsto & (1+\xi)^{-1} R_{\gamma}(1+\xi)+\lambda-\gamma .
\end{array}
$$

En effet, en notant 1 l'identité de $\mathbb{R}^{2}$, on commence par appliquer $\phi_{1}$ pour obtenir $\phi_{1}(\xi, \lambda)=(1+\xi, \lambda)$, puis on applique $\Phi_{\gamma}$ et on a

$$
\Phi_{\gamma} \phi_{1}(\xi, \lambda)=(1+\xi)^{-1} R_{\gamma}(1+\xi)+\lambda
$$

et enfin $\phi_{R_{\gamma}}^{-1} \Phi_{\gamma} \psi_{1}(\xi, \lambda)$ nous donne l'expression voulue. Bien entendu, ceci n'est valable que pour $\xi$ suffisamment petit et dans la suite, c'est cette expression de $\Phi_{\gamma}$ que l'on considère. On vérifie que c'est une bonne application de classe $C^{\infty}$ entre bons espaces de Fréchet (essentiellement parce que la composition et l'inverse sont de bonnes applications de classe $C^{\infty}$, voir l'annexe C).

(b) On calcule la différentielle de $\Phi_{\gamma}$.

Posons $(1+\mu)=(1+\xi)^{-1}$. Soit $(\hat{\xi}, \hat{\lambda})$ appartenant à $\Gamma^{\infty}\left(\mathbb{T}^{2}\right) \times \mathbb{R}^{2}$. En appliquant soigneusement la règle de chaîne il vient

$$
\begin{aligned}
d \Phi_{\gamma}(\xi, \lambda) \cdot(\hat{\xi}, \hat{\lambda})(x)= & d(1+\mu)\left(R_{\gamma}(1+\xi)(x)\right) \cdot \hat{\xi}(x) \\
& -d(1+\mu)\left(R_{\gamma}(1+\xi)(x)\right) \cdot \hat{\xi}\left((1+\mu) R_{\gamma}(1+\xi)(x)\right) \\
& +\hat{\lambda} .
\end{aligned}
$$

En particulier, on a $d \Phi_{\gamma}(0,0) \cdot(\hat{\xi}, \hat{\lambda})=\hat{\xi}-\hat{\xi} R_{\gamma}+\hat{\lambda}$.

(c) On réduit l'inversion de $d \Phi_{\gamma}$ à la résolution d'une équation cohomologique.

Rappelons que l'on cherche à inverser $d \Phi_{\gamma}$ au voisinage de $(0,0)$, i.e. on veut résoudre l'équation

$$
d \Phi_{\gamma}(\xi, \lambda) \cdot(\hat{\xi}, \hat{\lambda})=\eta
$$

où $\xi, \lambda, \eta$ sont données et $\hat{\xi}, \hat{\lambda}$ sont inconnues. On va alors transformer cette équation de telle sorte qu'elle soit "proche" de l'équation obtenue au point $(\xi, \lambda)=(0,0)$. On introduit alors les variables

$$
\begin{gathered}
\tilde{\xi}=\hat{\xi}(1+\mu) \\
\tilde{\eta}=d(1+\mu)\left((1+\mu) R_{\gamma}\right) \cdot(\eta(1+\mu))
\end{gathered}
$$

et

$$
\chi(\xi)=d(1+\mu)\left((1+\mu) R_{\gamma}\right)
$$


Il n'est pas difficile de vérifier que l'équation précédente devient

$$
\tilde{\xi}-\tilde{\xi} R_{\gamma}=\tilde{\eta}-\chi(\xi) \cdot \hat{\lambda}
$$

où les inconnues sont $\tilde{\xi}$ et $\hat{\lambda}$.

(d) On résout l'équation cohomologique.

Pour un élément $x=\left(x_{1}, x_{2}\right)$ dans $\mathbb{T}^{2}$, l'équation à résoudre s'écrit

$$
\tilde{\xi}(x)-\tilde{\xi}(x+\gamma)=\tilde{\eta}(x)-\chi(\xi)(x) \cdot \hat{\lambda} .
$$

Si $\xi$ est suffisamment petit, la matrice $M=\int_{\mathbb{T}^{2}} \chi(\xi)(x) d x$ est proche de l'identité donc inversible. Le terme de gauche a clairement une moyenne nulle pour la mesure de Haar sur le tore, ceci détermine donc $\hat{\lambda}=M^{-1} \int_{\mathbb{T}^{2}} \tilde{\eta}(x) d x$. On veut maintenant trouver $\tilde{\xi}$. Notons $\left(a_{k}\right)_{k \in \mathbb{Z}^{2}}$ ses coefficients de Fourier vis-à-vis de la mesure de Haar du tore, i.e.

$$
\tilde{\xi}(x)=\sum_{k \in \mathbb{Z}^{2}} a_{k} e^{2 \pi i k \cdot x} \quad \text { avec } \quad a_{k}=\int_{\mathbb{T}^{2}} \tilde{\xi}(x) e^{-2 \pi i k \cdot x} d \mu(x)
$$

et $\left(b_{k}\right)_{k \in \mathbb{Z}^{2}}$ ceux de $\tilde{\eta}-\chi(\xi) \cdot \hat{\lambda}$. Le choix de $\hat{\lambda}$ nous donne $b_{0}=a_{0}=0$. Pour $k \neq 0$, on a par identification

$$
a_{k}=\frac{b_{k}}{1-e^{2 \pi i k \cdot \gamma}}
$$

C'est ici qu'intervient la condition diophantienne sur $\gamma$. Elle nous donne une estimation du type

$$
\left|a_{k}\right| \leq C\left|b_{k}\right||k|^{2+\tau} \quad \forall k \in \mathbb{Z}^{2}
$$

pour une constante $C$ qui ne dépend que de $\gamma$. Puisque la fonction $\tilde{\eta}-\chi(\xi) . \hat{\lambda}$ est $C^{\infty}$, ses coefficients $\left(b_{k}\right)_{k \in \mathbb{Z}^{2}}$ sont à décroissance rapide à l'infini, il en est alors de même pour les coefficients $\left(a_{k}\right)_{k \in \mathbb{Z}^{2}}$. Il en résulte alors que la fonction $\tilde{\xi}$ est bien déterminée et $C^{\infty}$, et que l'application

$$
\begin{array}{ccc}
C^{\infty}\left(\mathbb{T}^{2}\right) & \longrightarrow & C^{\infty}\left(\mathbb{T}^{2}\right) \\
\Sigma_{k \in \mathbb{Z}^{2}} b_{k} e^{2 \pi i k .} & \longmapsto & \Sigma_{k \in \mathbb{Z}^{2}} a_{k} e^{2 \pi i k .}
\end{array}
$$

est une bonne application linéaire continue (avec une perte de différentiabilité $r=2+\tau)$.

On a donc réussi à construire une application $(\xi, \lambda, \eta) \longmapsto(\hat{\xi}, \hat{\lambda})$ qui est bien une section de $d \Phi_{\gamma}(\xi, \lambda)$ au voisinage de $(0,0)$. Cette application est bonne car elle est composée de bonnes applications : c'est clair pour $(\xi, \lambda, \eta) \longmapsto \hat{\lambda}$ et cela résulte du caractère diophantien de $\gamma$ pour $(\xi, \lambda, \eta) \longmapsto \hat{\xi}$. On conclut alors la preuve en utilisant le théorème d'inversion locale de Nash-Moser-Hamilton. 
Démonstration du théorème 2.1.1. Notons $N\left(\mathbb{T}^{2}\right)$ le sous-groupe normal engendré par les translations $\mathbb{T}^{2}$. D'après le lemme, il est ouvert au voisinage d'une translation diophantienne dans $D$ if $f_{0}^{\infty}\left(\mathbb{T}^{2}\right)$, donc c'est un sousgroupe ouvert. En particulier, il est fermé et par connexité de Dif $f_{0}^{\infty}\left(\mathbb{T}^{2}\right)$, on a l'égalité $N\left(\mathbb{T}^{2}\right)=$ Dif $f_{0}^{\infty}\left(\mathbb{T}^{2}\right)$. On a les inclusions

$$
\mathbb{T} \subseteq H \subseteq D i f f_{0}^{\infty}(\mathbb{T})
$$

où $\mathbb{T}$ agit sur le cercle par rotations et $H \cong P S L(2, \mathbb{R})$ agit sur le cercle par homographies, après identification du cercle avec la droite projective réelle $\mathbb{R} P^{1}$. Ainsi on a

$$
\mathbb{T}^{2} \subseteq H^{2} \subseteq \operatorname{Diff} f_{0}^{\infty}\left(\mathbb{T}^{2}\right)
$$

Cependant le groupe $H$ est parfait (même simple, c'est un résultat bien connu, voir [Lan02] par exemple) d'où

$$
\begin{aligned}
\operatorname{Diff} f_{0}^{\infty}\left(\mathbb{T}^{2}\right)=N\left(\mathbb{T}^{2}\right) & \subseteq N\left(H^{2}\right)=N\left(\left[H^{2}, H^{2}\right]\right) \\
& \subseteq\left[\text { Diff } f_{0}^{\infty}\left(\mathbb{T}^{2}\right), \text { Diff } f_{0}^{\infty}\left(\mathbb{T}^{2}\right)\right] .
\end{aligned}
$$

Le groupe $\operatorname{Dif} f_{0}^{\infty}\left(\mathbb{T}^{2}\right)$ est donc parfait.

Remarque 2.1.8. A cause du phénomène de pertes de dérivées, l'argument de Sergeraert-Herman tombe en défaut si l'on cherche la simplicité du groupe Dif $f_{0}^{r}\left(\mathbb{T}^{2}\right)$ pour $r$ fini. Cependant, par approximation des difféomorphismes de classe $C^{r}$ ( $r$ fini) par des difféomorphismes de classe $C^{\infty}$ dans la topologie $C^{r}$, il résulte que les commutateurs de Dif $f_{0}^{r}\left(\mathbb{T}^{2}\right)$ sont denses dans Dif $f_{0}^{r}\left(\mathbb{T}^{2}\right)$ pour la topologie $C^{r}$. Par le théorème d'Epstein, on en déduit que le groupe Dif $f_{0}^{r}\left(\mathbb{T}^{2}\right)$ est topologiquement simple, i.e. il ne contient pas de sous-groupe normal non trivial qui soit fermé.

Toujours par un argument de densité (cette fois-ci plus compliqué), on peut démontrer la simplicité du groupe des difféomorphismes analytiques du tore isotopes à l'identité ([Her75]).

Expliquons maintenant comment un raisonnement analogue nous donne le résultat un peu plus fort suivant : le groupe $D i \widehat{f f_{0}^{\infty}\left(\mathbb{T}^{2}\right)}$, revêtement universel de Dif $f_{0}^{\infty}\left(\mathbb{T}^{2}\right)$, est parfait.

Il est bon de commencer par donner quelques précisions sur le revêtement universel $\widetilde{G}$ d'un groupe topologique connexe $G$. Pour peu que $G$ soit localement contractile, $\widetilde{G}$ s'identifie aux classes d'homotopies (à extrémités fixes) d'isotopies dans $G$, où une isotopie dans $G$ est un chemin continu émanant de l'identité. Si $G$ possède une structure différentiable, on suppose que le chemin est lisse. L'ensemble $\widetilde{G}$ est muni d'une structure de groupe que l'on peut décrire de deux manières équivalentes : pour $g=\left(g^{t}\right)_{t \in[0,1]}$ 
et $h=\left(h^{t}\right)_{t \in[0,1]}$ deux isotopies dans $G$, on définit leur produit soit en concaténant les chemins, i.e.

$$
g * h: t \in[0,1] \longmapsto\left\{\begin{array}{lll}
h^{2 t} & \text { si } & 0 \leq t \leq 1 / 2 \\
g^{2 t-1} h^{1} & \text { si } & 1 / 2 \leq t \leq 1
\end{array}\right.
$$

(et éventuellement on reparamètre ce dernier pour qu'il soit lisse), soit en utilisant la structure de groupe de $G$, i.e.

$$
g . h: t \in[0,1] \longmapsto g^{t} h^{t} .
$$

On peut alors vérifier que $[g * h]=[g . h]$ dans $\widetilde{G}$, et que cela définit bien une loi de groupe.

De part sa structure différentiable, le groupe Diff $f^{\infty}\left(\mathbb{T}^{2}\right)$ est localement contractile. Ainsi, un élément de $D i \widehat{f f_{0}^{\infty}\left(\mathbb{T}^{2}\right)}$ est une classe d'homotopie à extrémités fixes $[\Phi]$ où

$$
\Phi: t \in[0,1] \longmapsto \varphi^{t} \in \operatorname{Diff} f_{0}^{\infty}\left(\mathbb{T}^{2}\right)
$$

est un chemin (lisse) de difféomorphismes du tore qui part de l'identité.

Proposition 2.1.9. Le groupe Dif $\widetilde{f f_{0}^{\infty}\left(\mathbb{T}^{2}\right)}$ est parfait.

Démonstration. Prenons $\gamma$ un vecteur diophantien dans $\mathbb{T}^{2}$ et soit

$$
s: V \longrightarrow \operatorname{Diff} f_{0}^{\infty}\left(\mathbb{T}^{2}\right) \times \mathbb{T}^{2}
$$

l'inverse local de l'application $\Phi_{\gamma}$ définie dans le théorème 2.1.7, $V$ étant un voisinage ouvert de la translation de vecteur $\gamma$. Par connexité, il suffit de montrer que toute isotopie $\Phi$ appartenant à $D i \widehat{f f_{0}^{\infty}\left(\mathbb{T}^{2}\right)}$ suffisamment proche de l'identité est un produit de commutateurs de $D i \widehat{f f_{0}^{\infty}\left(\mathbb{T}^{2}\right)}$. Choisissons alors $\Phi=\left(\varphi^{t}\right)_{t \in[0,1]}$ dans Dif $\widetilde{f_{0}^{\infty}\left(\mathbb{T}^{2}\right)}$ tel que $R_{\gamma} \varphi^{t} \in V \subseteq$ Dif $f_{0}^{\infty}\left(\mathbb{T}^{2}\right)$ pour tout $t \in[0,1]$. On applique le théorème de SergeraertHerman à $R_{\gamma} \varphi^{t}$ pour obtenir

$$
R_{\gamma} \varphi^{t}=R_{\lambda_{t}}\left(\psi^{t}\right)^{-1} R_{\gamma} \psi^{t}
$$

avec $s\left(\varphi^{t}\right)=\left(\psi^{t}, \lambda_{t}\right)$, pour tout $t \in[0,1]$. Puisque l'on peut, à $t$ fixé, passer continûment de $R_{\gamma}$ à $R_{t \gamma}$, notre isotopie $\Phi$ est homotope (à extrémités fixes) à l'isotopie

$$
t \in[0,1] \longmapsto R_{t \gamma}^{-1} R_{\lambda_{t}}\left(\psi^{t}\right)^{-1} R_{t \gamma} \psi^{t}=R_{\lambda_{t}}\left[R_{t \gamma}^{-1},\left(\psi^{t}\right)^{-1}\right]
$$

et cette dernière est certainement un produit de commutateurs. 


\subsection{Simplicité du groupe $\operatorname{Dif} f_{0}^{\infty}(M)$}

On considère maintenant une surface fermée $M$ quelconque. La tâche difficile qui nous attend est d'étendre le théorème de simplicité d'Herman au groupe Dif $f_{0}^{\infty}(M)$. La preuve de ce résultat est essentiellement due à Thurston et Mather, mais elle ne semble pas avoir été publiée par ces auteurs (voir [Thu74a] pour le papier original qui ne contient aucune preuve et [Mat79] pour une rédaction des idées de Thurston sur un résultat bien plus général, qui sera expliqué plus loin). Dans cette section, on suit le plan de [Ban97], tout en simplifiant certains arguments.

Dans un premier temps, on utilise des propriétés générales du groupe Dif $f_{0}^{\infty}(M)$ pour se ramener à l'étude du groupe des difféomorphismes à support dans $U, U$ étant un ouvert de $M$. Précisément, en notant Dif $f_{U}^{\infty}(M)$ ce dernier, on va montrer que s'il est parfait, alors le groupe Dif $f_{0}^{\infty}(M)$ est simple. Il faut cependant commencer par vérifier la propriété de fragmentation pour le groupe $\operatorname{Dif} f_{0}^{\infty}(M)$. La preuve est beaucoup plus facile que pour les homéomorphismes (voir [PS70]).

Théorème 2.2.1. Dif $f_{0}^{\infty}(M)$ a la propriété de fragmentation.

Démonstration. Soit $\mathcal{U}=\left\{U_{1}, \ldots, U_{m}\right\}$ un recouvrement ouvert de $M$ et $\lambda_{1}, \ldots, \lambda_{m}$ une partition de l'unité lisse associée à ce recouvrement.

Soit $g$ un élément de Dif $f_{0}^{\infty}(M)$. Pour démontrer la propriété, on peut le choisir arbitrairement proche de l'identité. A l'aide d'une carte exponentielle au voisinage de l'identité, on peut même choisir une isotopie

$$
\left(g^{t}=\exp \left(t \exp ^{-1} g\right)\right)_{t \in[0,1]}
$$

qui soit proche de l'identité. Définissons, pour $0 \leq k \leq m$, des fonctions $\mu_{k}=\Sigma_{i \leq k} \lambda_{i}$ et des difféomorphismes

$$
g_{k}(x)=g^{\mu_{k}(x)}(x) .
$$

Il est clair que $g_{k}$ est une application lisse. De plus, puisque $g_{k}$ dépend de manière continue de $g$ (et de l'isotopie $\left.\left(g^{t}\right)_{t \in[0,1]}\right)$, on peut toujours choisir $g$ suffisamment petit pour que $g_{k}$ reste proche de l'identité. Ainsi, $g_{k}$ est un difféomorphisme par le théorème d'inversion locale. Par définition des $\mu_{k}$, il est clair que $g_{k}(x)=g_{k-1}(x)$ si $x$ n'appartient pas à $U_{k}$. Donc $h_{k}=g_{k}\left(g_{k-1}\right)^{-1}$ est un difféomorphisme à support dans $U_{k}$, et on a

$$
g=g_{m}=\left(g_{m}\left(g_{m-1}\right)^{-1}\right)\left(g_{m-1}\left(g_{m-2}\right)^{-1}\right) \ldots\left(g_{1}\left(g_{0}\right)^{-1}\right)=h_{m} \ldots h_{1} .
$$

Remarque 2.2.2. La preuve précédente nous donne en fait un résultat plus fort, à savoir la propriété de fragmentation pour les isotopies. En effet, avec 
les notations précédentes, on définit une isotopie $\left(g_{k}^{t}\right)_{t \in[0,1]}$ pour $0 \leq k \leq m$ en posant

$$
g_{k}^{t}(x)=g^{t \mu_{k}(x)}(x) \quad \forall t \in[0,1], \forall x \in M
$$

et l'on vérifie que

$$
\left(g^{t}\right)=\left(h_{m}^{t}\right) \ldots\left(h_{1}^{t}\right)
$$

avec $h_{k}^{t}=g_{k}^{t}\left(g_{k-1}^{t}\right)^{-1}$ pour $1 \leq k \leq m$.

Remarque 2.2.3. La propriété de fragmentation est également valable, avec la même preuve, pour le groupe des difféomorphismes de classe $C^{r}$ (r fini) d'une variété compacte.

En fait, ce résultat combiné au théorème d'Epstein (voir l'annexe B) suffit à prouver que si le groupe $\operatorname{Dif} f_{U}^{\infty}(M)$ est parfait, alors le groupe Dif $f_{0}^{\infty}(M)$ est simple. En effet, par le théorème de fragmentation 2.2.1, la perfection de $\operatorname{Dif} f_{U}^{\infty}(M)$ implique celle de $D$ if $f_{0}^{\infty}(M)$ et le théorème d'Epstein nous assure donc que ce dernier est simple.

Il existe cependant une preuve beaucoup plus directe, due à Thurston, qui permet d'obtenir le même résultat sans utiliser le théorème d'Epstein. L'intérêt principal de cet argument est que, contrairement à celui d'Epstein, il est encore valable (sans aucune modification) dans le cas conservatif (voir la section 3.4). On aura besoin de la transitivité de l'action de Dif $f_{0}^{\infty}(M)$ $\operatorname{sur} M$ (voir [Ban97]).

Lemme 2.2.4. Dif $f_{0}^{\infty}(M)$ agit transitivement sur $M$. En particulier, ses orbites sont denses.

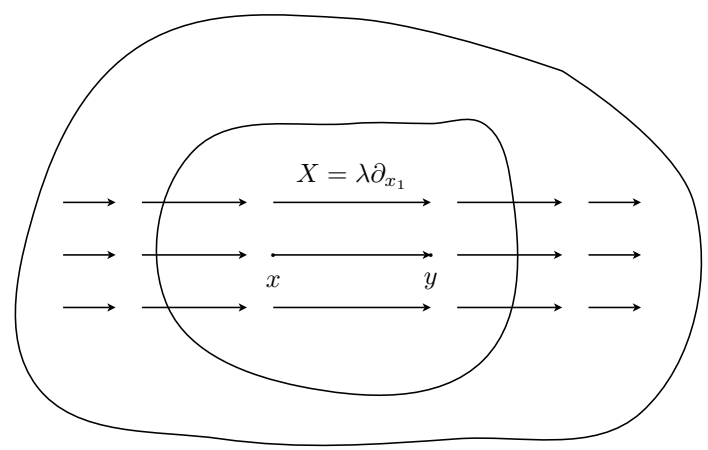

FIG. 2.1 - Transitivité de Dif $f_{0}^{\infty}(M)$ 
Démonstration. Soient $x$ et $y$ deux points distincts de $M$, on cherche un élément $g$ appartenant à $D$ if $f_{0}^{\infty}(M)$ tel que $g(x)=y$. Si $x$ et $y$ sont proches, on peut trouver une carte $\varphi: U \rightarrow \mathbb{R}^{2}$ telle que $\varphi(x)=(0,0)$ et $\varphi(y)=(0, \varepsilon)$ pour $\varepsilon>0$. Si $\lambda$ est une fonction plateau convenablement choisie, alors le temps $1 \mathrm{du}$ flot de $X=\lambda \partial_{x_{1}}$ est un difféomorphisme du plan qui envoie $\varphi(x)$ sur $\varphi(y)$, et en le conjuguant par $\varphi$, on obtient $g$ dans Dif $f_{0}^{\infty}(M)$ tel que $g(x)=y$ (voir la figure 2.1). Si maintenant $x$ et $y$ sont arbitraires, on prend un chemin $c$ les reliant que l'on découpe par compacité en un nombre fini de chemins suffisamment petits pour que l'on puisse appliquer l'argument précédent.

On utilisera souvent dans la suite le corollaire immédiat suivant.

Corollaire 2.2.5. Soit $U$ un ouvert dans $M$. Alors pour tout ouvert $V$ suffisamment petit, il existe un difféomorphisme $\varphi_{V}$ dans Dif $f_{0}^{\infty}(M)$ qui envoie $V$ dans $U$.

Théorème 2.2.6 (Thurston). Si Dif $f_{U}^{\infty}(M)$ est parfait, alors Dif $f_{0}^{\infty}(M)$ est simple.

Démonstration. Notons $G=\operatorname{Dif} f_{0}^{\infty}(M)$ et $G_{U}=\operatorname{Diff} f_{U}^{\infty}(M)$. La simplicité de $D$ if $f_{0}^{\infty}(M)$ va alors résulter uniquement des propriétés de fragmentation et de transitivité.

Soit $g$ un élément de $G$ différent de l'identité, et notons $N(g)$ le sousgroupe normal engendré par $g$. Il s'agit de prouver que $N(g)=G$. Soit $x$ un point non fixé par $g$. Puisque $G$ agit transitivement sur $M$, on peut trouver $h$ dans $G$ tel que $h(x)$ soit distinct de $x$ et $g(x)$. On se donne alors un ouvert $U$, qui est un voisinage de $x$ assez petit pour que $U, g(U)$ et $h(U)$ soient disjoints. Il est clair que pour tout $u, v \in G_{U}$, on a

$$
\begin{aligned}
& {[u, g]= \begin{cases}u & \text { sur } \mathrm{U} \\
g u^{-1} g^{-1} & \text { sur } \mathrm{g}(\mathrm{U}) \\
1 & \text { ailleurs }\end{cases} } \\
& {[v, h]= \begin{cases}v & \text { sur } \mathrm{U} \\
h v^{-1} h^{-1} & \text { sur h(U) } \\
1 & \text { ailleurs }\end{cases} }
\end{aligned}
$$

et une simple vérification donne $[u, v]=[[u, g],[v, h]]$. On a alors

$$
G_{U} \subseteq\left[G_{U}, G_{U}\right] \subseteq\left[\left[G_{U}, g\right],\left[G_{U}, h\right]\right] \subseteq[N(g), G] \subseteq N(g)
$$

où la première inclusion vient de l'hypothèse de perfection sur $G_{U}$. Pour $x$ appartenant à $M$, on trouve par transitivité un petit ouvert $U_{x}$ contenant $x$ et un élément $\alpha_{x}$ appartenant à $G$ tels que $\alpha_{x}\left(U_{x}\right)$ soit inclus dans $U$. On a alors, pour tout $x$ dans $M$,

$$
G_{U_{x}} \subseteq \alpha_{x}^{-1} G_{U} \alpha_{x} \subseteq N(g)
$$


Ces ouverts $U_{x}$ recouvrent $M$ et ainsi par fragmentation $\bigcup_{x \in M} G_{U_{x}}$ engendre $G$, ce qui donne $G \subseteq N(g)$.

En vue d'une utilisation ultérieure, énonçons le résultat précédent de manière plus synthétique, comme cela est fait dans [Hal98].

Théorème 2.2.7. Soient $X$ un espace topologique paracompact, $\mathcal{O}$ une base d'ouverts de $X$ un sous-groupe de Homeo $(X)$. Pour chaque ouvert $U$ dans $\mathcal{O}$, soit $G_{U}$ un sous-groupe de $\mathrm{Homeo}_{U}(X)$, où $\mathrm{Homeo}_{U}(X)$ est le sous-groupe des homéomorphismes à support dans $U$. Supposons que

(i) $G$ possède la propriété de fragmentation : si $\mathcal{U} \subseteq \mathcal{O}$ est un recouvrement de $X, \bigcup_{U \in \mathcal{U}} G_{U}$ engendre $G$;

(ii) $G$ agit transitivement sur les points de $X$;

(iii) pour tous $U, V \in \mathcal{O}$ et $g \in G$ tels que $g(U) \subseteq V, g G_{U} g^{-1} \subseteq G_{V}$. Si $G_{U}$ est parfait pour tout $U \in \mathcal{O}$, alors $G$ est simple.

Remarque 2.2.8. Dans la preuve précédente, on avait $G_{U}=G \cap H_{o m e o}(X)$ et dans ce cas l'hypothèse (iii) est trivialement vérifiée.

Lorsqu'on le compare au théorème d'Epstein, les hypothèses du théorème précédent ont l'avantage d'être plus claires (on n'a pas besoin de la condition supplémentaire qu'impose Epstein dans son axiome de fragmentation (E3)) et plus faciles à vérifier (on a seulement besoin d'une action transitive sur les points et non sur les disques, voir l'annexe B). De plus, comme on l'a déjà précisé, ce résultat est beaucoup plus flexible et il sera utilisé dans le prochain chapitre pour des groupes de difféomorphismes conservatifs.

Il nous reste à prouver que le groupe des difféomorphismes de $M$ à support dans un disque $U$ est parfait. Il suffit bien sûr de le faire pour une seule variété, car ce groupe est toujours isomorphe au groupe des difféomorphismes du plan à support compact. Si l'on prend $M=\mathbb{T}^{2}$ et $g$ un difféomorphisme du tore dont le support est dans $U$, on sait d'après le théorème de Herman que $g$ est un produit de commutateurs dans Dif $f_{0}^{\infty}(M)$, mais il faut alors montrer que c'est un produit de commutateurs dans $\operatorname{Dif} f_{U}^{\infty}(M)$. Il est peut-être possible d'écrire une preuve directe de ce fait, mais pour des raisons techniques on va se placer sur l'abélianisé du revêtement universel de ce groupe, i.e. on va considérer le groupe des isotopies (à homotopies près) quotienté par les isotopies de commutateurs. Comme on va le voir dans la suite, ce groupe abélien peut se décrire comme le premier groupe d'homologie d'un certain complexe de chaînes, et il sera alors possible d'utiliser le formalisme de l'homologie.

Commençons par quelques préliminaires. Notons $G=D$ if $f_{0}^{\infty}(M)$. On va utiliser des notions d'homologie cubique dont on peut trouver une exposition détaillée dans [Mas80]. On désigne par $K^{n}=[0,1]^{n}$ le $n$-cube unité, et on ordonne ses sommets selon l'ordre lexicographique. Un $n$-cube singulier est une application continue

$$
c: K^{n} \longrightarrow G \text {. }
$$


On dit que le $n$-cube singulier est normalisé lorsque $c$ envoie le premier sommet $v_{0}$ de $K_{n}$ sur l'identité de $G$, et qu'il est dégénérée lorsque l'application $c$ ne dépend que d'au plus $n-1$ variables. On définit alors $B \bar{G}_{n}$ comme le $\mathbb{Z}$-module libre engendré par les $n$-cubes singuliers normalisés, quotienté par le sous-module des $n$-cubes singuliers normalisés dégénérés. Lorsque $c$ est un $n$-cube singulier non normalisé, on note $\hat{c}$ sa normalisation, i.e. $\hat{c}=c\left(v_{0}\right)^{-1} c$. Dans la suite, on utilisera plus simplement le terme $n$-cube pour désigner un $n$-cube singulier normalisé.

On définit un opérateur de bord $\partial: B \bar{G}_{n} \rightarrow B \bar{G}_{n-1}$ en prenant la restriction aux $(n-1)$-faces et en normalisant : précisément, si $c$ est un $n$-cube, on définit pour $1 \leq i \leq n$ et pour $\varepsilon=0,1$, les côtés

$$
\left(F_{i}^{\varepsilon} c\right)\left(t_{1}, \ldots, t_{n-1}\right)=c\left(t_{1}, \ldots, t_{i-1}, \varepsilon, t_{i}, \ldots, t_{n-1}\right)
$$

puis la $i$-ème face de $c$

$$
\partial_{i} c=\widehat{F_{i}^{0} c}-\widehat{F_{i}^{1} c}
$$

et enfin le bord de $c$ est

$$
\partial c=\sum_{i=1}^{n}(-1)^{i} \partial_{i} c .
$$

On peut alors vérifier que $\partial^{2}=0$.

Exemple 2.2.9. Un 1-cube n'est rien d'autre qu'une isotopie c dans $G$, et on $a$

$$
\partial c=\widehat{F_{1}^{0} c}-\widehat{F_{1}^{1}} c=c(0)-\left(c(1)^{-1}\right) c(1)=0
$$

donc les 1-cubes sont automatiquement des 1-cycles. Pour un 2-cube c, on a (voir la figure 2.2)

$$
\begin{aligned}
(\partial c)(t) & =\widehat{F_{1}^{1}} c(t)-\widehat{F_{1}^{0}} c(t)+\widehat{F_{2}^{0}} c(t)-\widehat{F_{2}^{1}} c(t) \\
& =\left(c(0,1)^{-1}\right) c(t, 1)-c(t, 0)+c(0, t)-\left(c(1,0)^{-1}\right) c(1, t) .
\end{aligned}
$$

Soit $B \bar{G}$ la réunion des $B \bar{G}_{n}$, on obtient ainsi un complexe de chaîne $(B \bar{G}, \partial)$ dont on note $H_{*}(B \bar{G}, \mathbb{Z})$ l'homologie à coefficients entiers. C'est ce qu'on appelle l'homologie locale du groupe $G$.

Remarque 2.2.10. Le terme "homologie locale" provient du fait que l'homologie du complexe $B \bar{G}$ peut se calculer avec des petits cubes, i.e. des cubes contenus dans un voisinage de l'identité. Par exemple, si $G$ est un groupe de Lie, on a $H_{1}(B \bar{G}) \cong \mathfrak{g} /[\mathfrak{g}, \mathfrak{g}]$ où $\mathfrak{g}$ est son algèbre de Lie de $G$ (voir [Hae78] par exemple).

En ce qui nous concerne, l'intérêt de cette notion réside dans la proposition suivante. 


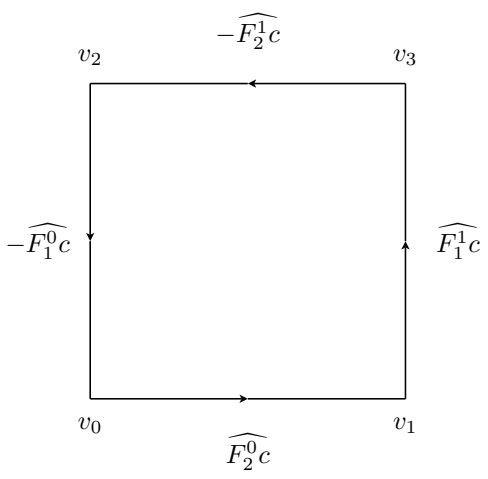

Fig. 2.2 - Bord d'un 2-cube

Proposition 2.2.11. On a un isomorphisme de groupes

$$
\widetilde{G} /[\widetilde{G}, \widetilde{G}] \cong H_{1}(B \bar{G}, \mathbb{Z}) .
$$

En particulier, $\widetilde{G}$ est parfait si et seulement si $H_{1}(B \bar{G}, \mathbb{Z})$ est trivial.

On va se contenter de décrire l'isomorphisme en question, qui est analogue à l'isomorphisme de Hurewicz entre le premier groupe d'homologie singulière et l'abélianisé du groupe fondamental d'un espace topologique.

Soit $c$ une isotopie dans $G$, i.e. $c:[0,1] \rightarrow G$ est un chemin tel que $c(0)=$ $e$. On note $[c]$ sa classe d'homotopie dans $\widetilde{G}$ et $[[c]]$ sa classe d'homologie dans $H_{1}(B \bar{G}, \mathbb{Z})$. Remarquons que cette dernière définition est correcte puisque $c$, en tant qu'élément de $B \bar{G}_{1}$, est un 1-cycle. Définissons alors

$$
\begin{aligned}
& \Phi: \widetilde{G} \longrightarrow H_{1}(B \bar{G}, \mathbb{Z}) \\
& {[c] \longmapsto \quad[[c]]}
\end{aligned}
$$

et montrons que cette application a un sens. Soit $c^{\prime}$ tel que $\left[c^{\prime}\right]=[c]$, alors $c^{\prime}$ et $c$ sont des isotopies homotopes à extrémités fixes, et on note $H:[0,1]^{2} \rightarrow G$ une telle homotopie. Alors $H$ est un 2-cube, qui est constant sur $\{0\} \times[0,1]$ et $\{1\} \times[0,1]$, donc $\partial_{1} H=0$ (voir la figure 2.3). On a donc

$$
\partial H=\partial_{2} H=c-c^{\prime}
$$

dans $B \bar{G}_{1}$, i.e. $\left[\left[c^{\prime}\right]\right]=[[c]]$. Ainsi $\Phi$ est bien définie.

On peut maintenant vérifier que c'est un morphisme de groupes. Prenons $c$ et $c^{\prime}$ deux éléments de $\widetilde{G}$, et $c * c^{\prime}$ leur produit. On définit alors un 2-cube singulier

$$
\sigma: K\left(v_{0}, v_{1}, v_{2}, v_{3}\right) \longrightarrow G
$$




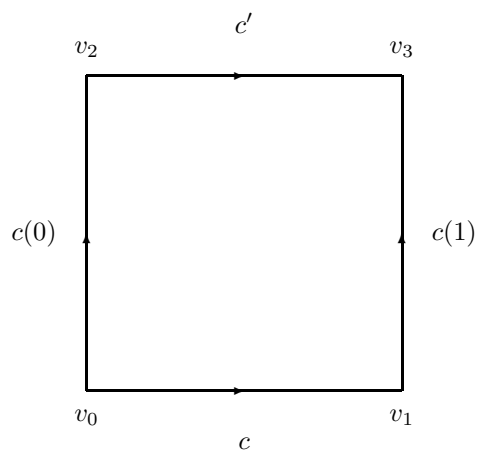

FIG. $2.3-[c]=\left[c^{\prime}\right] \Rightarrow[[c]]=\left[\left[c^{\prime}\right]\right]$

de la manière suivante : on pose $\sigma_{\mid\left[v_{0}, v_{1}\right]}=c, \sigma_{\mid\left[v_{1}, v_{2}\right]}=c(1), \sigma_{\mid\left[v_{2}, v_{3}\right]}=$ $c(1) c^{\prime}$ et on étend de manière continue $\sigma$ à l'intérieur de $K\left(v_{0}, v_{1}, v_{2}, v_{3}\right)$ pour que $\sigma_{\mid\left[v_{0}, v_{3}\right]}=c * c^{\prime}$ (voir la figure 2.4, on demande que $\sigma$ soit constante sur chaque segment oblique et que $\sigma$ soit constante égale à $c(1)$ ailleurs). On obtient ainsi

$$
\partial \sigma=c * c^{\prime}-c^{\prime}-c
$$

dans $B \bar{G}_{1}$ et on a bien $\left[\left[c * c^{\prime}\right]\right]=[[c]]+\left[\left[c^{\prime}\right]\right]$. On se retrouve donc avec un morphisme de groupes

$$
\Phi: \widetilde{G} \longrightarrow H_{1}(B \bar{G}, \mathbb{Z})
$$

Puisque $H_{1}(B \bar{G}, \mathbb{Z})$ est abélien, $\Phi$ envoie les commutateurs de $\widetilde{G}$ sur l'élément neutre de $H_{1}(B \bar{G}, \mathbb{Z})$, donc elle passe au quotient

$$
\Phi: \widetilde{G} /[\widetilde{G}, \widetilde{G}] \longrightarrow H_{1}(B \bar{G}, \mathbb{Z}) .
$$

On peut alors montrer que $\Phi$ est un isomorphisme, et cela se fait de la même manière que dans le théorème de Hurewicz (voir par exemple [May92]).

Remarque 2.2.12. Dans la section suivante, on donnera une interprétation plus abstraite de l'homologie locale d'un groupe.

Étant données $c$ et $c^{\prime}$ deux isotopies dans $G$, on peut désormais soit les concaténer, soit les composer ou bien encore considérer leur somme en homologie. D'après ce qui précède, ces opérations coïncident dans $\widetilde{G} /[\widetilde{G}, \widetilde{G}] \cong$ $H_{1}(B \bar{G}, \mathbb{Z})$. Le lemme suivant est alors évident.

Lemme 2.2.13. Soient $\tau$ une isotopie dans $G$ et $g$ un élément de $G$. Alors $\tau$ et $g \tau g^{-1}$ sont égaux dans $H_{1}(B \bar{G}, \mathbb{Z})$. 


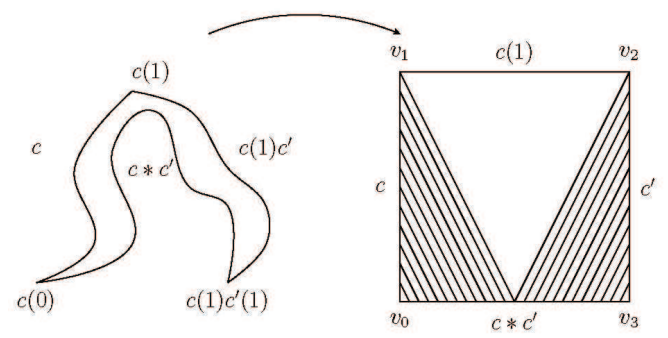

FIG. $2.4-\left[\left[c * c^{\prime}\right]\right]=[[c]]+\left[\left[c^{\prime}\right]\right]$

On aura également besoin du résultat suivant, dont on peut trouver une preuve dans [Hir76].

Lemme 2.2.14. Soient $V, U$ deux disques de $M$ et $f, g$ deux plongements préservant l'orientation de $V$ dans $U$. Alors il existe une isotopie $\left(\varphi^{t}\right)_{t \in[0,1]}$ à support dans $U$ telle que $f \varphi^{1}=g$.

On est maintenant en mesure d'énoncer et de prouver le théorème de "déformation" de Thurston-Mather.

Théorème 2.2.15 (Thurston-Mather). Soit $U$ un ouvert de $M$. Alors l'inclusion $G_{U} \subseteq G$ induit un isomorphisme

$$
H_{1}\left(B \overline{G_{U}}, \mathbb{Z}\right) \cong H_{1}(B \bar{G}, \mathbb{Z}) .
$$

Expliquons tout d'abord comment ce théorème implique la simplicité du groupe Dif $f_{0}^{\infty}(M)$. D'après ce qui précède, $H_{1}(B \bar{G}, \mathbb{Z})=0$ si et seulement si $\widetilde{G}$ est parfait. En appliquant le résultat de Thurston-Mather à $M=\mathbb{T}^{2}$, on a un isomorphisme de groupes

$$
H_{1}\left(B \overline{\operatorname{Diff} f_{U}^{\infty}\left(\mathbb{T}^{2}\right)}, \mathbb{Z}\right) \cong H_{1}\left(B \overline{\operatorname{Diff} f_{0}^{\infty}\left(\mathbb{T}^{2}\right)}, \mathbb{Z}\right)
$$

puis par le théorème d'Herman, on obtient

$$
H_{1}\left(B \overline{D i f f_{U}^{\infty}\left(\mathbb{T}^{2}\right)}, \mathbb{Z}\right) \cong 0 .
$$

Donc $\widetilde{\operatorname{Diff}_{U}^{\infty}}\left(\mathbb{T}^{2}\right)$ est parfait, ce qui nous donne aussitôt la perfection de Dif $f_{U}^{\infty}\left(\mathbb{T}^{2}\right)$, puis celle de Dif $f_{U}^{\infty}(M)$ pour une surface $M$ quelconque. On obtient ainsi la simplicité de $\operatorname{Dif} f_{0}^{\infty}(M)$ dans le cas général en appliquant l'astuce de Thurston 2.2.7.

Démonstration du théorème 2.2.15. Notons

$$
\rho: B \overline{G_{U}} \longrightarrow B \bar{G}
$$


l'application induite par l'inclusion $G_{U} \subseteq G$ et

$$
\rho_{*}: H_{1}\left(B \overline{G_{U}}, \mathbb{Z}\right) \longrightarrow H_{1}(B \bar{G}, \mathbb{Z})
$$

le morphisme induit en homologie. On note [[.]] les classes d'homologie de $H_{1}(B \bar{G}, \mathbb{Z})$ et $[[.]]_{U}$ celles de $H_{1}\left(B \overline{G_{U}}, \mathbb{Z}\right)$. La surjectivité de ce morphisme sera une conséquence facile du lemme de fragmentation 2.2.1 pour les isotopies, mais c'est de l'injectivité dont on a vraiment besoin. Pour cela, il faudra établir un lemme de fragmentation pour les 2-cubes dans $G$, i.e. pour les familles de difféomorphismes à deux paramètres.

\section{(a) Surjectivité de $\rho_{*}$.}

Soit $\alpha$ appartenant à $H_{1}(B \bar{G}, \mathbb{Z})=\widetilde{G} /[\widetilde{G}, \widetilde{G}]$, et soit $g=\left(g^{t}\right)_{t \in[0,1]}$ une isotopie représentant $\alpha$. On va alors découper $g$ en une somme finie d'isotopies à support dans $U$ pour trouver un antécédent $\beta \in H_{1}\left(B \overline{G_{U}}, \mathbb{Z}\right)$. Soit $\mathcal{U}=\left\{U_{1}, \ldots, U_{m}\right\}$ un recouvrement ouvert suffisamment fin pour que l'on puisse trouver, par transitivité, des $h_{i}$ appartenant à $G$ qui envoient $U_{i}$ dans $U$ pour $1 \leq i \leq m$. On fragmente alors l'isotopie $g$ pour obtenir

$$
\left(g^{t}\right)=\left(g_{1}^{t}\right) \ldots\left(g_{n}^{t}\right)
$$

avec, pour $1 \leq j \leq n,\left(g_{j}^{t}\right)_{t \in[0,1]}$ une isotopie à support dans l'un des $U_{i}$ (voir la remarque 2.2.2). Si on note $\alpha_{j}$ la classe d'homologie de $\left(g_{j}^{t}\right)_{t \in[0,1]}$ dans $H_{1}(B \bar{G}, \mathbb{Z})$, on a l'égalité

$$
\alpha=\alpha_{1}+\cdots+\alpha_{n} .
$$

Maintenant l'isotopie $\left(h_{i} g_{j}^{t} h_{i}^{-1}\right)_{t \in[0,1]}$ est à support dans $U$, notons $\beta_{j}$ sa classe d'homologie dans $H_{1}\left(B \overline{G_{U}}, \mathbb{Z}\right)$. Il est alors clair que

$$
\alpha=\rho_{*}\left(\beta_{1}+\cdots+\beta_{n}\right) .
$$

En effet, il suffit de remarquer que par le lemme 2.2.13, les isotopies $\left(g_{j}^{t}\right)_{t \in[0,1]}$ et $\left(h_{i} g_{j}^{t} h_{i}^{-1}\right)_{t \in[0,1]}$ coïncident dans $H_{1}(B \bar{G}, \mathbb{Z})$, i.e. $\alpha_{j}=\rho_{*} \beta_{j}$ pour $1 \leq j \leq$ $n$. On en déduit ainsi la surjectivité.

\section{(b) Un lemme de fragmentation.}

Commençons par quelques notations. Si on se donne un entier $m$, on peut subdiviser le $n$-cube unité $[0,1]^{n}$ en $m^{n}$ cubes

$$
K_{k_{1}, \ldots, k_{n}}=\left\{\left(t_{1}, \ldots, t_{n}\right) \in[0,1]^{n} \mid k_{i} / m \leq t_{i} \leq\left(k_{i}+1\right) / m, 1 \leq i \leq n\right\} .
$$

On dispose d'une identification canonique

$$
h_{k_{1}, \ldots, k_{n}}:[0,1]^{n} \longrightarrow K_{k_{1}, \ldots, k_{n}}
$$


qui envoie le sommet $(0, \ldots, 0)$ sur $\left(k_{1} / m, \ldots, k_{n} / m\right)$. Ainsi, si on se donne un $n$-cube $c:[0,1]^{n} \rightarrow G$, on peut lui associer par composition un $n$-cube

$$
c_{k_{1}, \ldots, k_{n}}=c_{\mid K_{k_{1}, \ldots, k_{n}} \circ h_{k_{1}, \ldots, k_{n}}}:[0,1]^{n} \longrightarrow G .
$$

Il est alors clair que $c$ est homologue à la $n$-chaîne $\sum c_{k_{1}, \ldots, k_{n}}$, et en choisissant l'entier $m$ suffisamment grand, on montre ainsi que le groupe $H_{n}(B \bar{G}, \mathbb{Z})$ est engendré par les $n$-cubes $C^{1}$-proches de l'identité. Remarquons que le lemme de fragmentation 2.2.1 peut s'interpréter de la manière suivante : si $\mathcal{U}=\left\{U_{1}, \ldots, U_{m}\right\}$ est un recouvrement ouvert de $M$ et $c:[0,1] \rightarrow G$ un 1-cube $C^{1}$-proche de l'identité, alors il existe un 1-cube $\tilde{c}$ qui vérifie les deux conditions suivantes :

(i) on a $[[\tilde{c}]]=[[c]]$;

(ii) le 1-cube $\tilde{c}_{k}$ est à support dans $U_{k}$, pour $0 \leq k \leq m$.

Pour prouver l'injectivité, on aura besoin du lemme suivant, qui est une version "à deux paramètres" du lemme de fragmentation 2.2.1.

Lemme 2.2.16. Soient $\mathcal{U}=\left\{U_{1}, \ldots, U_{m}\right\}$ un recouvrement ouvert de $M$ et $c:[0,1]^{2} \rightarrow G$ un 2 -cube $C^{1}$-proche de l'identité. Alors il existe un 2 -cube $\tilde{c}$ qui vérifie les trois conditions suivantes :

(i) si $\partial$ c est à support dans $U$, alors $\partial \tilde{c}$ aussi et $[[\partial \tilde{c}]]_{U}=[[\partial c]]_{U}$;

(ii) chaque 2 -cube $\tilde{c}_{k_{1}, k_{2}}$ est à support dans $U_{k_{1}+1} \cup U_{k_{2}+1}$;

(iii) si $U_{k_{1}+1} \cap U_{k_{2}+1}=\emptyset$, alors $\partial \tilde{c}_{k_{1}, k_{2}}=0$.

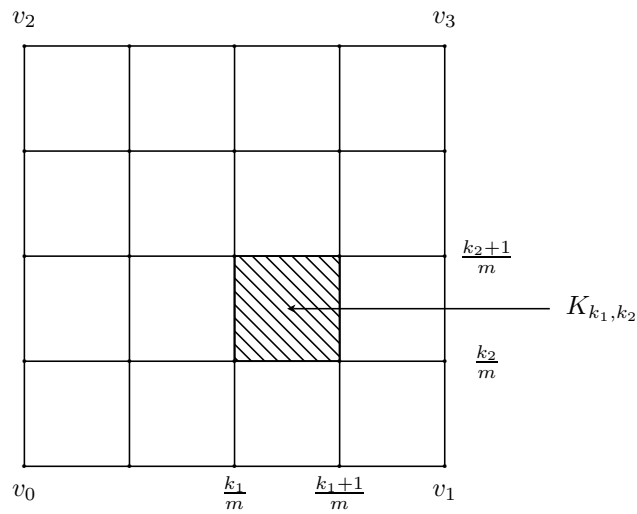

FIG. 2.5 - Décomposition de $[0,1]^{2}$

Démonstration. On se donne une partition de l'unité $\left\{\lambda_{1}, \ldots, \lambda_{m}\right\}$ associée au recouvrement $\left\{U_{1}, \ldots, U_{m}\right\}$ et on introduit, pour $0 \leq k_{1}, k_{2} \leq m$, des 
fonctions $\mu_{k_{1}}=\Sigma_{i \leq k_{1}} \lambda_{i}$ et $\mu_{k_{2}}=\Sigma_{i \leq k_{2}} \lambda_{i}$. Subdivisons notre carré $[0,1]^{2}$ en $m^{2}$ carrés $K_{k_{1}, k_{2}}$ (voir la figure 2.5) et définissons une application lisse

$$
\begin{aligned}
& f:[0,1]^{2} \times M \quad \longrightarrow \quad[0,1]^{2} \\
& (t, s, x) \longmapsto f_{x}(t, s)
\end{aligned}
$$

de la manière suivante : en chaque sommet $\left(k_{1} / m, k_{2} / m\right)$ de la subdivision, on pose

$$
f_{x}\left(k_{1} / m, k_{2} / m\right)=\left(\mu_{k_{1}}(x), \mu_{k_{2}}(x)\right)
$$

puis on étend $f_{x}$ à $[0,1]^{2}$ de manière affine sur chaque carré $K_{k_{1}, k_{2}}$ de la subdivision (il faut penser à cette application comme à un reparamétrage qui dépend du point). Remarquons que notre application $f_{x}$ fixe les quatre sommets de $[0,1]^{2}$. Considérons $c$ un 2-cube $C^{1}$-proche de l'identité, au sens où $c(t, s)$ est $C^{1}$-proche de l'application identité de $M$ pour tout $(t, s)$ dans $[0,1]^{2}$. Alors la formule

$$
\tilde{c}(t, s) \cdot(x)=c\left(f_{x}(t, s)\right) \cdot(x)
$$

définit un 2-cube $\tilde{c}$. En effet, $\tilde{c}(s, t)$ est un difféomorphisme car c'est une application lisse $C^{1}$-proche de l'identité pour tout $(t, s)$ dans $[0,1]^{2}$, et on a $\tilde{c}(0,0)=e$.

Il nous reste à démontrer que $\tilde{c}$ a bien les propriétés requises. Par définition de $\tilde{c}$, si $\partial c$ est à support dans un disque $U$, il en est de même pour $\partial \tilde{c}$. Écrivons

$$
\partial \tilde{c}=\widehat{F_{1}^{1}} \tilde{c}-\widehat{F_{1}^{0} \tilde{c}}+\widehat{F_{2}^{0} \tilde{c}}-\widehat{F_{2}^{1} \tilde{c}} .
$$

Pour $i=1,2, \varepsilon=0,1$ et $x \in M$, remarquons que la restriction de $f_{x}$ aux côtés de $[0,1]^{2}$ fixe les sommets et est homotope à l'identité, ainsi l'isotopie $\widehat{F_{i}^{\varepsilon} \tilde{c}}$ est homotope à extrémités fixes à $\widehat{F_{i}^{\varepsilon} c}$ par une homotopie à support dans $U$, ce qui nous donne l'égalité

$$
[[\partial \tilde{c}]]_{U}=[[\partial c]]_{U}
$$

Vérifions maintenant que le support de $\tilde{c}_{k_{1}, k_{2}}$ est inclus dans l'union $U_{k_{1}+1} \cup$ $U_{k_{2}+1}$. Si $x$ n'est pas dans $U_{k_{1}+1} \cup U_{k_{2}+1}$, on obtient facilement que la restriction de $f_{x}$ à $K_{k_{1}, k_{2}}$ est constante (car constante sur les quatre sommets de $\left.K_{k_{1}, k_{2}}\right)$, donc par normalisation $\tilde{c}_{k_{1}, k_{2}}(x)=x$, ce qui signifie bien que $x$ n'appartient pas au support de $\tilde{c}_{k_{1}, k_{2}}$. Enfin, si $U_{k_{1}+1} \cap U_{k_{2}+1}=\emptyset$, on obtient que pour tout $x$ dans $M$, l'application $f_{x}$ est constante sur au moins deux côtés opposés de $K_{k_{1}, k_{2}}$, et puisque elle est affine sur chaque côté de $K_{k_{1}, k_{2}}$, on en déduit que $\widehat{F_{2}^{0} \tilde{c}_{k_{1}, k_{2}}}=\widehat{F_{2}^{1} \tilde{c}_{k_{1}, k_{2}}}$ et $\widehat{F_{1}^{1} \tilde{c}_{k_{1}, k_{2}}}=\widehat{F_{1}^{0} \tilde{c}_{k_{1}, k_{2}}}$ et donc $\partial \tilde{c}_{k_{1}, k_{2}}=0$. Ceci achève la preuve du lemme.

Remarque 2.2.17. De manière un peu plus générale, si on considère dans le lemme précédent une 2-chaîne

$$
c=\sum_{j \in J} c^{j}
$$


avec des 2-cubes $c^{j} C^{1}$-proches de l'identité et telle que $\partial c$ soit à support dans $U$, bien que chaque élément $\partial c^{j}$ ne le soit pas nécessairement (c'est ce qui se produit lorsqu'il y a des compensations dans la somme $\left.\sum_{j \in J} \partial c^{j}\right)$, alors en notant

$$
\tilde{c}=\sum_{j \in J} \tilde{c}^{j}
$$

on montre de même que $\partial \tilde{c}$ est à support dans $U$ et que $[[\partial \tilde{c}]]_{U}=[[\partial c]]_{U}$. Dans la suite, il faudra faire attention au fait que

$$
\left[\left[\sum_{j \in J} \partial c^{j}\right]\right]_{U} \neq \sum_{j \in J}\left[\left[\partial c^{j}\right]\right]_{U}
$$

puisque le terme de droite peut ne pas avoir de sens.

(c) Injectivité de $\rho_{*}$.

Soit $z$ un 1-cycle dans $B \overline{G_{U}}$ bordant une 2-chaîne dans $B \bar{G}$ (i.e. $[[z]]=$ $\left.\rho_{*}[[z]]_{U}=0\right)$, on veut donc prouver que $z$ borde une 2-chaîne dans $B \overline{G_{U}}$ (i.e. $\left.[[z]]_{U}=0\right)$.

Soit $c=\sum_{j \in J} c^{j}$ une 2-chaîne dans $B \bar{G}$ telle que $\partial c=z$. Quitte à subdiviser, on suppose que $c^{j}$ est $C^{1}$-proche de l'identité, pour tout $j$ dans $J$. Étant donné un recouvrement ouvert $\left\{U_{1}, \ldots, U_{m}\right\}$ (que l'on choisira dans la suite), soit $\tilde{c}^{j}$ le 2-cube construit dans le lemme précédent à partir du 2-cube $c^{j}$. Définissons une 2-chaîne

$$
\tilde{c}=\sum_{j \in J} \sum_{k_{1}, k_{2}} \tilde{c}_{k_{1}, k_{2}}^{j}
$$

et posons $\tilde{z}=\partial \tilde{c}$. D'après le lemme 2.2 .16 et la remarque 2.2 .17 , on sait que $\partial \tilde{c}$ est à support dans $U$ et que

$$
[[\tilde{z}]]_{U}=[[\partial \tilde{c}]]_{U}=[[\partial c]]_{U}=[[z]]_{U} .
$$

Il est donc suffisant de prouver que $[[\tilde{z}]]_{U}=0$. On sait de plus que $\tilde{c}_{k_{1}, k_{2}}^{j}$ est à support dans $U_{k_{1}+1} \cup U_{k_{2}+1}$ et $\partial \tilde{c}_{k_{1}, k_{2}}^{j}=0$ si cette union est disjointe. Il est alors naturel de définir le sous-ensemble d'indices

$$
K^{\prime}=\left\{\left(k_{1}, k_{2}\right) \mid U_{k_{1}+1} \cap U_{k_{2}+1} \neq \emptyset\right\}
$$

et de poser

$$
\bar{c}=\sum_{j \in J} \sum_{\left(k_{1}, k_{2}\right) \in K^{\prime}} c_{k_{1}, k_{2}}^{j} .
$$

On obtient ainsi $\tilde{z}=\partial \bar{c}$, autrement dit $\tilde{z}$ borde une 2-chaîne constituée de 2-cubes, chacun ayant support dans une union de deux ouverts de $\mathcal{U}$ d'intersection non vide. 
Maintenant soit $\mathcal{V}$ une famille de disques ouverts recouvrant $M$, telle que si $V_{i}, V_{j} \in \mathcal{V}$, alors leur intersection $V_{i} \cap V_{j}$, si elle est non vide, est difféomorphe à un disque (de tels recouvrements existent, voir [KN63]). On choisit alors notre recouvrement $\mathcal{U}$ de telle sorte que si l'intersection de deux ouverts de $\mathcal{U}$ est non vide, alors leur réunion est contenue dans un certain élément de $\mathcal{V}$. Ainsi $\tilde{z}$ borde la 2-chaîne

$$
\bar{c}=\sum_{j \in J^{\prime}} \bar{c}_{j}
$$

(on introduit un nouvel ensemble d'indice $J^{\prime}$ pour alléger les notations) avec le support de $\bar{c}_{j}$ inclus dans $V_{j} \in \mathcal{V}$, après réindexation et pour $j$ dans $J^{\prime}$.

En notant $\tau_{j}^{i}$ les "côtés" orientés de $\bar{c}_{j}$, on a

$$
\tilde{z}=\partial \bar{c}=\sum_{j} \partial \bar{c}_{j}=\sum_{i, j} \tau_{j}^{i} .
$$

Pour chaque 1-cube $\tau_{j}^{i}$, définissons un élément $h_{j}^{i} \in G$ de la manière suivante : si le support de $\tau_{j}^{i}$ est dans $U$, on prend pour $h_{j}^{i}$ l'identité, sinon $\tau_{j}^{i}$ est à support dans une intersection $V_{j j^{\prime}}=V_{j} \cap V_{j^{\prime}}$ (car dans ce cas $\tau_{j}^{i}$ est un côté commun à des 2-cubes distincts $\bar{c}_{j}$ et $\left.\bar{c}_{j^{\prime}}\right)$ et alors $h_{j}^{i}$ est un élément de $G$ qui envoie cette intersection dans $U$. Ainsi le 1 -cube $h_{j}^{i} \tau_{j}^{i}\left(h_{j}^{i}\right)^{-1}$ est à support dans $U$, et on a l'égalité

$$
\tilde{z}=\sum_{i, j} \tau_{j}^{i}=\sum_{i, j} h_{j}^{i} \tau_{j}^{i}\left(h_{j}^{i}\right)^{-1}
$$

En effet, puisque $\tilde{z}$ est à support dans $U$, ou bien $\tau_{j}^{i}$ est déjà à support dans $U$ et dans ce cas $h_{j}^{i}$ est l'identité, ou bien $\tau_{j}^{i}$ se compense avec un terme $\tau_{j^{\prime}}^{i^{\prime}}$ dans la somme de gauche pour $(i, j) \neq\left(i^{\prime}, j^{\prime}\right)$, dans ce cas on a pu choisir $h_{j}^{i}=h_{j^{\prime}}^{i^{\prime}}$ et les termes $h_{j}^{i} \tau_{j}^{i}\left(h_{j}^{i}\right)^{-1}$ et $h_{j^{\prime}}^{i^{\prime}} \tau_{j^{\prime}}^{i^{\prime}}\left(h_{j^{\prime}}^{i^{\prime}}\right)^{-1}$ se compensent dans la somme de droite. Encore par transitivité, on trouve pour tout indice $j$ un élément $g_{j}$ dans $G$ qui envoie $V_{j}$ dans $U$. La 2-chaîne $\sum_{j} g_{j} \bar{c}_{j} g_{j}^{-1}$ est à support dans $U$, et son bord est

$$
w=\partial\left(\sum_{j} g_{j} \bar{c}_{j} g_{j}^{-1}\right)=\sum_{i, j} g_{j} \tau_{j}^{i} g_{j}^{-1} .
$$

Pour conclure, il suffit de prouver que pour tout $i, j$, les isotopies $h_{j}^{i} \tau_{j}^{i}\left(h_{j}^{i}\right)^{-1}$ et $g_{j} \tau_{j}^{i} g_{j}^{-1}$ sont homologues dans $B \overline{G_{U}}$, car on aurait alors $[[\tilde{z}]]_{U}=[[w]]_{U}=$ 0 . On note $\tilde{h}_{j}^{i}$ et $\tilde{g}_{j}$ les restrictions de $h_{j}^{i}$ et de $g_{j}$ à $V_{j j^{\prime}}$. Les isotopies $\tilde{h}_{j}^{i} \tau_{j}^{i}\left(\tilde{h}_{j}^{i}\right)^{-1}$ et $\tilde{g}_{j} \tau_{j}^{i} \tilde{g}_{j}^{-1}$ sont défini respectivement sur $h_{j}^{i}\left(V_{j j^{\prime}}\right)$ et $g_{j}\left(V_{j j^{\prime}}\right)$, et quitte à les étendre par l'identité ailleurs, on a

$$
h_{j}^{i} \tau_{j}^{i}\left(h_{j}^{i}\right)^{-1}=\tilde{h}_{j}^{i} \tau_{j}^{i}\left(\tilde{h}_{j}^{i}\right)^{-1} \quad ; \quad g_{j} \tau_{j}^{i} g_{j}^{-1}=\tilde{g}_{j} \tau_{j}^{i} \tilde{g}_{j}^{-1} .
$$


Maintenant $\tilde{h}_{j}^{i}$ et $\tilde{g}_{j}$ sont des plongements préservant l'orientation du disque $V_{j j^{\prime}}$ dans $U$, par le lemme 2.2.14 il existe une isotopie $\Phi=\left(\varphi^{t}\right)_{t \in[0,1]}$ à support dans $U$ telle que

$$
\varphi^{1} \tilde{h}_{j}^{i}=\tilde{g}_{j}
$$

Ainsi on obtient

$$
g_{j} \tau_{j}^{i} g_{j}^{-1}=\tilde{g}_{j} \tau_{j}^{i} \tilde{g}_{j}^{-1}=\varphi^{1} \tilde{h}_{j}^{i} \tau_{j}^{i}\left(\tilde{h}_{j}^{i}\right)^{-1}\left(\varphi^{1}\right)^{-1}=\varphi^{1} h_{j}^{i} \tau_{j}^{i}\left(h_{j}^{i}\right)^{-1}\left(\varphi^{1}\right)^{-1}
$$

et puisque $\varphi^{1} h_{j}^{i} \tau_{j}^{i}\left(h_{j}^{i}\right)^{-1}\left(\varphi^{1}\right)^{-1}$ est homologue dans $B \overline{G_{U}}$ à $h_{j}^{i} \tau_{j}^{i}\left(h_{j}^{i}\right)^{-1}$ (par le lemme 2.2.13), on en déduit finalement que $g_{j} \tau_{j}^{i} g_{j}^{-1}$ est homologue dans $B \overline{G_{U}}$ à $h_{j}^{i} \tau_{j}^{i}\left(h_{j}^{i}\right)^{-1}$. Ceci achève la preuve du théorème.

Remarque 2.2.18. La preuve du théorème 2.2.15 repose principalement sur la transitivité de l'action de $G$ sur $M$ (lemme 2.2.4), la fragmentation pour les 1-cubes (lemme 2.2.1) et pour les 2-cubes (lemme 2.2.16) ainsi que sur le lemme 2.2.14. Dans le prochain chapitre, on va étendre ces résultats au cas symplectique.

Remarque 2.2.19. On peut même obtenir un résultat plus général, à savoir un isomorphisme

$$
H_{k}\left(B \overline{G_{U}}, \mathbb{Z}\right) \cong H_{k}(B \bar{G}, \mathbb{Z})
$$

pour tout $k \geq 1$ (voir la section suivante).

On a donc prouvé que le groupe des difféomorphismes $C^{\infty}$ isotopes à l'identité d'une surface fermée est simple. Il nous reste encore à examiner le cas ou le degré de différentiabilité est fini, mais avant cela, on va s'autoriser une petite digression dans la section suivante afin d'énoncer la théorie de Thurston-Mather en toute généralité.

\section{$2.3 \quad$ Feuilletages et espaces classifiants}

Dans cette section, on souhaite mentionner un lien entre l'homologie locale des groupes de difféomorphismes et l'étude des feuilletages. Cependant, la théorie étant difficile, on ne donnera aucune preuve (ni même des idées de preuves), le but ici étant seulement d'informer le lecteur sur certaines questions qui ont motivé l'étude de la perfection des groupes de difféomorphismes. On trouvera des démonstrations dans [Law77], [Mat79] et [Ser78] et on pourra consulter [Hae] et [Tsu] pour des présentations plus informelles.

Commençons par quelques rappels. Plusieurs définitions des feuilletages sont possibles, en voici une adaptée à notre contexte. 
Définition 2.3.1. Un feuilletage $\mathcal{F}$ sur une variété $M$, de classe $C^{r}$ et de codimension $q$, est une famille maximale de $C^{r}$-submersions

$$
f_{i}: U_{i} \longrightarrow \mathbb{R}^{q}, i \in I
$$

où $\left(U_{i}\right)_{i \in I}$ est un recouvrement ouvert de $M$, qui vérifie la condition de compatibilité suivante : pour tout $i, j$ dans $I$ et $x$ dans $U_{i} \cap U_{j}$, il existe un difféomorphisme local $\phi_{j i}^{x}$ de $\mathbb{R}^{q}$ tel que $f_{j}=\phi_{j i}^{x} f_{i}$ au voisinage de $x$.

Les feuilles du feuilletages sont définies localement par les composantes connexes des fibres $f_{i}^{-1}(c)$, pour $i$ dans $I$ et $c$ appartenant à $\mathbb{R}^{q}$. Ce sont des sous-variétés (immergées) de $M$. À un feuilletage $\mathcal{F}$ sur $M$ on associe son fibré tangent $\tau(\mathcal{F})$, le fibré vectoriel tangent aux feuilles du feuilletages et son fibré normal $\nu(\mathcal{F})$ qui est défini par les fonctions de transitions $\left(d \phi_{j i}^{x}\right)_{i, j \in I}$. On a évidemment

$$
\nu(\mathcal{F}) \cong T M / \tau(\mathcal{F})
$$

Enfin, les difféomorphismes locaux $\phi_{j i}^{x}$, pour $x$ appartenant à $M$ et $i, j$ dans $I$, définissent le pseudo-groupe d'holonomie du feuilletage.

Remarque 2.3.2. Il est intéressant de voir ce que ces définitions donnent dans un cas extrême. Si $\mathcal{F}$ est de codimension maximale, cela revient à dire que les $\left(f_{i}\right)_{i \in I}$ sont des difféomorphismes locaux, les feuilles de $\mathcal{F}$ sont les points de $M$, la structure de feuilletage n'est rien d'autre que la structure de variété de $M$ et son fibré normal est le fibré tangent $T M$.

En un sens trivial, il existe toujours des feuilletages sur les variétés. On pourrait alors se demander si étant donné un sous-fibré vectoriel $V$ de $T M$, il existe un feuilletage $\mathcal{F}$ tangent à $V$. En dimension 1, la question est évidente puisque tout champ de droites (de classe Lipschitz) est intégrable par le théorème d'existence de solutions d'équations différentielles. De manière générale, la réponse est donnée par un théorème de Frobenius : $V$ doit être intégrable, i.e. stable par crochet de Lie. Cette condition se révèle être trop restrictive, et la plupart des champs de plans ne sont pas intégrables. La question intéressante concernant l'existence de feuilletages est en fait la suivante.

Question 1. Soit $V$ un sous-fibré vectoriel de TM. Est-ce que $V$ est homotope au fibré tangent d'un feuilletage $\mathcal{F}$ ?

Dans le cas général, la réponse est négative puisque Bott a donné une obstruction topologique, à travers des classes caractéristiques, au problème d'existence précédent (voir [Bot70]). Cependant son argument ne marche que pour des feuilletages de codimension strictement plus grande que 1 . En codimension 1, la réponse est toujours positive et c'est une conséquence d'un théorème profond de Thurston que l'on va rencontrer dans la suite. C'est cette question sur l'existence de feuilletages qui est liée à l'homologie 
des groupes de difféomorphismes, mais avant d'y arriver, le chemin est encore long.

Commençons par reformuler la notion de feuilletage. On note $\Gamma_{q}^{r}$ l'ensemble des germes de $C^{r}$-difféomorphismes locaux de $\mathbb{R}^{q}$. Rappelons qu'un élément de $\Gamma_{q}^{r}$ est une classe d'équivalence $[\phi, x]$ où $x$ appartient à $M$, $\phi$ est un $C^{r}$-difféomorphisme local défini au voisinage de $x$ et la relation d'équivalence est $[\phi, x] \sim\left[\phi^{\prime}, x\right]$ si et seulement si $\phi$ et $\phi^{\prime}$ coïncident au voisinage de $x$. On notera plus simplement $\phi^{x}$ ou encore $\phi$ un représentant de $[\phi, x]$. Cet ensemble joue un rôle essentiel dans l'étude des feuilletages. Il possède une structure de groupoïde topologique, ce qui signifie les choses suivantes. On a des applications "source" et "cible"

$$
s, t: \Gamma_{q}^{r} \longrightarrow \mathbb{R}^{q}
$$

définies par $s\left(\phi^{x}\right)=x$ et $t\left(\phi^{x}\right)=\phi(x)$ et une application identité

$$
e: \mathbb{R}^{q} \longrightarrow \Gamma_{q}^{r}
$$

donnée par $e(x)=1^{x}$. Ces applications sont continues si l'on munit $\Gamma_{q}^{r}$ de sa topologie naturelle de faisceau, $s$ et $t$ sont des homéomorphismes locaux et $e$ un homéomorphisme sur son image. Pour $\phi, \psi$ appartenant à $\Gamma_{q}^{r}$, la composition est possible si $t(\phi)=s(\psi)$, elle est alors associative et continue. Enfin, tout élément $\phi \in \Gamma_{q}^{r}$ est inversible et $\phi \phi^{-1}=e(s(\phi))$, $\phi^{-1} \phi=e(t(\phi))$.

Remarque 2.3.3. De manière plus formelle, un groupoïde est une "petite catégorie" dont tous les morphismes sont inversibles : on a comme dans toute catégorie une collection d'objets Obj, un ensemble de morphismes Mor entre deux objets et des applications

$$
s, t: M o r \longrightarrow O b j
$$

ainsi qu'une opération de composition entre morphismes si la source de l'un coïncide avec la cible de l'autre. Mais de plus, on a une application

$$
e: \mathrm{Obj} \longrightarrow \mathrm{Mor}
$$

qui identifie Obj à une partie de l'ensemble des morphismes (et qui fait donc de Obj un ensemble). C'est ainsi l'ensemble des morphismes $\Gamma=$ Mor que l'on considère comme étant le groupoïde. Un morphisme entre deux groupoïdes n'est rien d'autre qu'un foncteur entre les deux catégories associées. Enfin, remarquons qu'un groupe est un groupoïde avec un seul objet.

Exemple 2.3.4 (Le groupoïde de recouvrement). Étant donné un recouvrement ouvert $\mathcal{U}=\left(U_{i}\right)_{i \in I}$ d'un espace $M$, on peut définir un groupoïde 
de la manière suivante : les objets sont par définition les ouverts du recouvrement et l'ensemble des morphismes est

$$
\Gamma_{\mathcal{U}}=\left\{(i, j, x) \mid i, j \in I, x \in U_{i} \cap U_{j}\right\} .
$$

La composition est définie par $(i, j, x)(j, k, x)=(i, k, x)$.

Voici comment on peut redéfinir les feuilletages en termes de cocycle à valeurs dans le groupoïde $\Gamma_{q}^{r}$. Soit $\mathcal{F}$ un feuilletage donné par une famille $\left(U_{i}, f_{i}\right)_{i \in I}$. Regardons alors l'application

$$
\phi_{j i}: U_{i} \cap U_{j} \longrightarrow \Gamma_{q}^{r}
$$

qui à $x$ associe $\left[\phi_{j i}^{x}, f_{i}(x)\right]$, le germe de $\phi_{j i}^{x}$ au point $f_{i}(x)$. On vérifie alors d'une part que $\phi_{j i}$ est continue, et d'autre part qu'on a la condition de cocycle suivante au voisinage de $x \in U_{i} \cap U_{j} \cap U_{k}$ :

$$
\phi_{j i}^{x}=\phi_{j k}^{x} \phi_{k i}^{x}
$$

qui nous garantit que les feuilletages "locaux" définis par les submersions $\left(f_{i}\right)_{i \in I}$ se recollent en un feuilletage global. En d'autres termes, notre famille $\left(U_{i}, f_{i}\right)_{i \in I}$ définit un morphisme de groupoïdes topologiques $\Gamma_{\mathcal{U}} \rightarrow \Gamma_{q}^{r}$, avec $\mathcal{U}=\left(U_{i}\right)_{i \in I}$. Sous cette forme, un feuilletage sur $M$ peut être considéré comme l'espace total d'un fibré principal au dessus de $M$ où la fibre, au lieu d'être un groupe topologique, est un groupoïde topologique. On espère pouvoir ainsi définir, par exemple, des espaces classifiants et des classes caractéristiques afin de comprendre les obstructions à l'existence de feuilletages sur certaines variétés.

Pour faire marcher ces théories de nature homotopique, il faut d'abord s'affranchir de toute hypothèse de différentiabilité et définir une notion de feuilletage "singulier" valable sur des espaces topologiques, et qui généralise la notion usuelle de feuilletage ainsi que celle de fibration principale. Dans la suite, on note $\Gamma=\Gamma_{q}^{r}$. On introduit alors naturellement la définition suivante.

Définition 2.3.5. Soit $X$ un espace topologique paracompact. Une structure de Haefliger sur $X$ à valeurs dans $\Gamma$ est une famille maximale $\left(U_{i}, \phi_{j i}\right)$ où $\mathcal{U}=\left(U_{i}\right)_{i \in I}$ est un recouvrement ouvert de $X$ et $\left(\phi_{j i}\right)_{j, i \in I}$ une famille d'applications continues

$$
\phi_{j i}: U_{i} \cap U_{j} \longrightarrow \Gamma
$$

qui vérifie la condition de cocycle

$$
\phi_{j i}^{x}=\phi_{j k}^{x} \phi_{k i}^{x}
$$

sur les intersections $U_{i} \cap U_{j} \cap U_{k}, \forall i, j, k \in I$. 
Remarque 2.3.6. La définition précédente a un sens pour un groupoïde topologique quelconque. Une structure de Haefliger sur un espace $X$ à valeurs dans $\Gamma$ est une classe d'équivalence de morphismes de groupoïdes topologiques $\Gamma_{\mathcal{U}} \rightarrow \Gamma$, où $\mathcal{U}$ est un recouvrement ouvert de $X$. Lorsque $\Gamma=G$ est un groupe, une structure de Haefliger sur $X$ à valeurs dans $G$ est une G-fibration principale.

Si $X$ est une variété lisse et si les applications

$$
f_{i}=s \phi_{i i}=t \phi_{i i}: U_{i} \longrightarrow \mathbb{R}^{q}
$$

sont des submersions pour tout $i$ dans $I$, on retrouve la définition des feuilletages. On note $H^{1}(X, \Gamma)$ l'ensemble des structures de Haefliger sur $X$. L'avantage essentiel que l'on a à considérer les structures de Haefliger est le suivant : si $Y$ est un espace topologique et $\mathcal{H} \in H^{1}(Y, \Gamma)$, une application continue $f: X \rightarrow Y$ induit automatiquement une structure de Haefliger $f^{*} \mathcal{H} \in H^{1}(X, \Gamma)$, ce qui est bien sûr faux pour les feuilletages sans une hypothèse de transversalité.

En vue de classifier les structures de Haefliger, il faut d'abord commencer par définir une relation d'équivalence convenable.

Définition 2.3.7. Deux structures de Haefliger $\mathcal{H}_{0}$ et $\mathcal{H}_{1}$ sur un espace $X$ sont concordantes (ou homotopes) s'il existe une structure de Haefliger $\mathcal{H}$ sur le produit $X \times[0,1]$ tel que $i_{t}^{*} \mathcal{H}=\mathcal{H}_{t}$, pour $t=0,1$, avec $i_{t}: X \hookrightarrow$ $X \times\{t\}$ le plongement canonique.

Si $Y$ est un espace topologique et $\mathcal{H} \in H^{1}(Y, \Gamma)$, deux applications homotopes $f_{0}, f_{1}: X \rightarrow Y$ induisent sur $X$ des structures de Haefliger concordantes. En fait, toute classe de concordance de structure de Haefliger sur $X$ s'obtient de la sorte : c'est le théorème de classification de Haefliger.

Théorème 2.3.8 (Haefliger). Il existe un espace topologique BГ muni d'une structure de Haefliger $\mathcal{H}_{\Gamma}$ qui classifie toutes les structures de Haefliger au sens suivant : pour tout espace topologique $X$, l'ensemble des classes de concordances de structures de Haefliger sur $X$ est en bijection avec l'espace des classes d'homotopies d'applications de $X$ dans $B \Gamma$.

La preuve originale de Haefliger ([Hae58]) repose sur le théorème de représentabilité de Brown. Notons qu'on peut donner des constructions explicites de l'espace $B \Gamma$ : voir [Law77] pour une méthode analogue au "joint infini" de Milnor ou [Tsu] pour une autre construction due à Segal.

Ainsi, toute structure de Haefliger sur un espace $X$ est induite au moyen d'une application classifiante $f: X \rightarrow B \Gamma$, i.e. est de la forme $f^{*}\left(\mathcal{H}_{\Gamma}\right)$ où $\mathcal{H}_{\Gamma}$ est la structure de Haefliger universelle sur $B \Gamma$. Cependant, l'intérêt de ce théorème de classification pour l'étude des feuilletages n'est pas clair. Il faudrait en effet pouvoir associer à chaque classe de concordance de structures de Haefliger une classe d'équivalence convenable de feuilletages. Commençons alors par la définition suivante. 
Définition 2.3.9. Deux feuilletages $\mathcal{F}_{0}$ et $\mathcal{F}_{1}$ sur une variété $M$ sont concordants s'il existe un feuilletage $\mathcal{F}$ sur le produit $M \times[0,1]$, transverse aux sous-variétés $M \times\{t\}$ pour $t=0,1$ et qui se restreint en $\mathcal{F}_{0}$ sur $M \times\{0\}$ et en $\mathcal{F}_{1}$ sur $M \times\{1\}$.

Il est clair que deux feuilletages concordants sont également concordants en tant que structures de Haefliger, mais la réciproque semble déraisonnable. En effet, supposons maintenant que $X=M$ est une variété lisse. À toute structure de Haefliger $\mathcal{H}$ sur $M$ on peut associer un certain fibré vectoriel $\nu(\mathcal{H})$, que l'on appelle fibré normal à $\mathcal{H}$. Si $\left(U_{i}, \phi_{j i}\right)_{i, j \in I}$ est un cocycle définissant $\mathcal{H}$, alors $\left(U_{i}, d \phi_{j i}\right)_{i, j \in I}$ est un cocycle à valeurs dans $G L(q, \mathbb{R})$ définissant ce fibré vectoriel $\nu(\mathcal{H})$. Si $\mathcal{H}$ est un feuilletage, il est immédiat que $\nu(\mathcal{H})$ est un sous-fibré de codimension $q$ du fibré vectoriel tangent $T M$ et de plus, si $\mathcal{H}$ et $\mathcal{H}^{\prime}$ sont concordants en tant que structures de Haefliger, alors les fibrés $\nu(\mathcal{H})$ et $\nu\left(\mathcal{H}^{\prime}\right)$ sont homotopes. Cela nous conduit à la définition suivante.

Définition 2.3.10. Une classe de concordance tangentielle de structures de Haefliger est la donnée d'une classe de concordance de structures de Haefliger et d'une classe d'homotopie de plongements $i: \nu(\mathcal{H}) \hookrightarrow T M$.

On peut alors énoncer le théorème fondamental de Thurston (voir [Law77] ou [Thu74b], [Thu76] pour les papiers originaux).

Théorème 2.3.11 (Thurston). Si $M$ est une variété compacte, les classes de concordances de feuilletages sur $M$ sont en bijection avec les classes de concordances tangentielles de structures de Haefliger.

Remarque 2.3.12. Dans le cas non compact, le théorème reste vrai sous une équivalence plus stricte (c'est un résultat de Gromov-Phillips-Haefliger).

En d'autres termes, si une structure de Haefliger sur $M$ a un fibré normal isomorphe à un sous-fibré vectoriel de $T M$, alors c'est un feuilletage à concordance près. La preuve de ce théorème (très difficile) repose sur la remarque suivante : on peut représenter toute classe de concordance de structure de Haefliger sur une variété $M$ par une fibration au dessus de $M$, de fibre $\mathbb{R}^{q}$, munie d'un feuilletage transverse aux fibres. Expliquons cette construction.

Rappelons qu'un fibré feuilleté au dessus de $M$ est une fibration dont l'espace total est muni d'un feuilletage transverse aux fibres et dont la projection restreint aux feuilles est un revêtement. Ces feuilletages possèdent alors un morphisme d'holonomie global

$$
h: \pi_{1}(M) \longrightarrow \operatorname{Diff}(F)
$$

où $F$ est la fibre type de la fibration (et réciproquement, tout fibré feuilleté est la suspension de son morphisme d'holonomie). Les fibrés feuilletés sont exactement les fibrés dont le groupe structural peut être réduit à un groupe 
discret. On parle de micro-fibré feuilleté lorsque l'espace total admet un feuilletage transverse aux fibres (ce qui est équivalent à l'existence d'une connexion plate) et qu'il existe une section distinguée qui permet d'identifier la base de la fibration à une feuille. On va associer à chaque structure de Haefliger sur $M$ un micro-fibré feuilleté (germinalement bien défini). Soit $\left(U_{i}, \phi_{j i}\right)$ un cocycle définissant la structure de Haefliger $\mathcal{H}$. Par compacité, on suppose que le recouvrement $\left(U_{i}\right)_{i \in I}$ est fini. Considérons les applications continues $f_{i}: U_{i} \rightarrow \mathbb{R}^{q}\left(f_{i}=s \phi_{i i}\right)$, et pour tout $i \in I$, soit $O_{i}$ un voisinage du graphe de $f_{i}$ dans $U_{i} \times \mathbb{R}^{q}$. On définit notre espace total

$$
E=\bigsqcup_{i \in I} O_{i} / \sim
$$

où la relation d'équivalence est donnée par $\left(x, y_{i}\right) \sim\left(x, y_{j}\right)$ si et seulement si $y_{j}=\phi_{j i}^{x}\left(y_{i}\right)$ pour $x \in U_{i} \cap U_{j}$. Quitte à choisir les voisinages $O_{i}$ suffisamment petits, la projection sur le premier facteur de $E$ lui donne une structure de fibré sur $M$, de fibre $\mathbb{R}^{q}$ ainsi qu'une section continue $s: M \rightarrow E$ donné localement par les $f_{i}, i \in I$. De plus, les feuilletages horizontaux de $U_{i} \times \mathbb{R}^{q}$ (de feuilles $U_{i} \times\{c\}$ pour $c \in \mathbb{R}^{q}$ ) se recollent en un feuilletage $\mathcal{F}_{\mathcal{H}}$ transverse aux fibres qui, considéré comme structure de Haefliger, vérifie $s^{*}\left(\mathcal{F}_{\mathcal{H}}\right)=\mathcal{H}$. Bien sûr, l'espace total $E$ n'est pas canoniquement défini, mais si on fait un autre choix $E^{\prime}$ avec une section $s^{\prime}$, on peut trouver un difféomorphisme d'un voisinage de $s$ sur un voisinage de $s^{\prime}$, qui envoie $s$ sur $s^{\prime}$, préserve les feuilletages sur l'espace total et respecte la projection sur la base (c'est ce qu'on entend par germinalement bien défini). On retrouve le fibré normal $\nu(\mathcal{H})$ en prenant les espaces tangents aux fibres le long de la section $s$.

Voici une illustration de la puissance du théorème précédent. Puisque tout fibré en droites réelles $L$ exhibe un feuilletage transverse aux fibres (son groupe structural $O(1) \cong \mathbb{Z} / 2 \mathbb{Z}$ étant discret), on peut voir $L$ comme le fibré normal d'une structure de Haefliger sur $M$ et par le théorème de Thurston, $L$ est homotope au fibré normal d'un feuilletage de codimension 1. Le corollaire suivant est alors immédiat.

Corollaire 2.3.13. Une variété compacte $M$ possède un feuilletage de codimension 1 si et seulement si sa caractéristique d'Euler-Poincaré est nulle.

On est maintenant en mesure d'utiliser l'espace classifiant $B \Gamma_{r}^{q}$ pour étudier l'existence de feuilletages. En prenant la différentielle d'un élément de $\Gamma_{r}^{q}$, on obtient une application $\Gamma_{r}^{q} \rightarrow G L(q, \mathbb{R})$ qui induit une application sur les espaces classifiants

$$
\nu: B \Gamma_{q}^{r} \longrightarrow B G L(q, \mathbb{R}) .
$$

Une manière plus abstraite de voir cette application est de remarquer que l'espace $B \Gamma_{r}^{q}$ a une structure de Haefliger (universelle), donc un fibré normal 
(de dimension $q$ ) qui est classifié par $B G L(q, \mathbb{R}): \nu$ n'est alors rien d'autre que l'application classifiante.

Le problème de l'existence de feuilletages sur $M$ se ramène ainsi à un problème de relèvement d'applications.

Question 2. Soit $\nu: B \Gamma_{q}^{r} \rightarrow B G L(q, \mathbb{R})$ l'application définie plus haut. Étant donnée une application continue $f: M \rightarrow B G L(q, \mathbb{R})$, admet-elle un relèvement $\tilde{f}: M \rightarrow B \Gamma_{q}^{r}$ tel que $f=\nu \tilde{f}$ ?

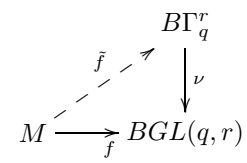

En effet, si l'on se donne un sous-fibré vectoriel $V$ de $T M$ de codimension $q$, son fibré normal $T M / V$ est classifié par une application continue $f$ : $M \rightarrow B G L(q, \mathbb{R})$, et le fait de pouvoir relever cette application à $B \Gamma_{q}^{r}$ signifie que ce fibré normal est celui d'une certaine structure de Haefliger. Le théorème de Thurston nous garantit alors que $V$ est homotope au fibré tangent d'un feuilletage. Il faut donc étudier ce problème de relèvement, mais pour cela, on aura besoin de quelques notions de topologie algébrique.

Soit $f: X \rightarrow Y$ une application continue entre espaces topologiques, et soit l'espace (le "mapping path space")

$$
X \times_{f} Y^{I}=\left\{(x, c) \in X \times Y^{I} \mid c(0)=f(x)\right\}
$$

avec $I=[0,1]$ et $Y^{I}$ l'ensemble des chemins continus $c: I \rightarrow Y$. On a alors une application continue

$$
\begin{aligned}
& \tilde{f}: X \times_{f} Y^{I} \longrightarrow Y \\
& (x, c) \longmapsto c(1)
\end{aligned}
$$

qui est une fibration (au sens où elle possède la propriété de relèvement des homotopies). La fibre homotopique d'un point $y$ appartenant à $Y$ est par définition l'ensemble $\tilde{f}^{-1}(y)$. On a une équivalence d'homotopie $h: X \rightarrow$ $X \times_{f} Y^{I}$ qui consiste à envoyer $x$ sur $\left(x, c_{x}\right)$ où $c_{x}$ est le chemin constant égale à $f(x)$, et qui vérifie $f=\tilde{f} h$. Ainsi toute application continue peut être considérée, à homotopie près, comme une fibration. Avec ce formalisme, on peut donner une manière plus abstraite de voir l'homologie locale d'un groupe (voir [Mat79] pour plus de détails). On suppose notre groupe $G$ localement contractile (ce qui est vérifié par tous les groupes que l'on considère dans ce texte). Soit $G_{\delta}$ le groupe $G$ muni de la topologie discrète et $i: G_{\delta} \rightarrow G$ l'application identité. Puisqu'elle est continue, on peut voir cette application comme une fibration, de fibre homotopique

$$
\bar{G}=\left\{(g, c) \in G_{\delta} \times G^{[0,1]} \mid c(0)=g, c(1)=e\right\} .
$$


Si l'on considère la topologie compacte-ouverte sur l'espace des chemins et la topologie discrète sur les points de départs, $\bar{G}$ devient naturellement un groupe topologique. On peut alors prouver que $B \bar{G}$, l'espace classifiant de $\bar{G}$, est une réalisation du complexe de chaînes que l'on a défini dans la section précédente et que par conséquent l'homologie singulière de $B \bar{G}$ n'est autre que l'homologie locale du groupe $G$.

Revenons maintenant à notre problème de relèvement, et notons $B \overline{\Gamma_{q}^{r}}$ la fibre homotopique de l'application $\nu: B \Gamma_{q}^{r} \rightarrow B G L(q, \mathbb{R})$. Par la théorie de l'obstruction (voir [Bre93]), l'existence d'un tel relèvement dépend de la topologie de l'espace $B \overline{\Gamma_{q}^{r}}$, plus précisément des groupes $H^{k}\left(X, \pi_{k-1}\left(B \overline{\Gamma_{q}^{r}}\right)\right)$ pour $k \geq 1$. On est donc amené à regarder la topologie de cet espace, qui comme on va le voir, est liée à l'homologie locale des groupes de difféomorphismes.

On se donne $G$ un sous-groupe de $\operatorname{Diff} f^{r}\left(\mathbb{R}^{q}\right)$ (on pourrait tout aussi bien prendre un sous-groupe de $\operatorname{Diff} f^{r}(M)$ où $M$ est une variété de dimension q). On a une fibration

$$
\bar{G} \longrightarrow G_{\delta} \longrightarrow G
$$

qui nous donne une fibration au niveau des espaces classifiants

$$
B \bar{G} \longrightarrow B G_{\delta} \longrightarrow B G
$$

De manière plus concrète, $B G$ classifie les $G$-fibrations, $B G_{\delta}$ classifie les $G$-fibrations plates (existence d'un feuilletage transverse aux fibres) et $B \bar{G}$ classifie les $G$-fibrations plates globalement triviales, i.e. les produits feuilletés $N \times \mathbb{R}^{q}$ dont l'holonomie est dans $G$. Si $G$ fixait un point (disons l'origine de $\mathbb{R}^{q}$ ) il y aurait un moyen naturelle de définir un morphisme de groupoïdes injectif

$$
G_{\delta} \longrightarrow \Gamma_{q}^{r}
$$

en envoyant $g \in G_{\delta}$ sur son germe à l'origine, puis on aurait alors une tour commutative

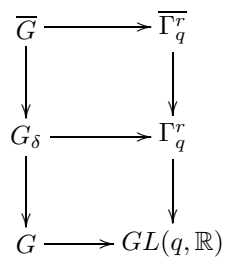


qui induirait au niveau des espaces classifiants

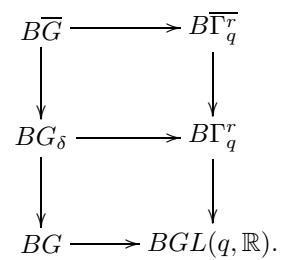

Mais dans le cas général, on ne peut pas obtenir de telles applications. L'astuce est alors de rajouter des paramètres, i.e. de considérer le produit $G_{\delta} \times \mathbb{R}^{q}$ et de définir un morphisme de groupoïdes

$$
G_{\delta} \times \mathbb{R}^{q} \longrightarrow \Gamma_{q}^{r}
$$

en envoyant un couple $(g, x) \in G_{\delta} \times \mathbb{R}^{q}$ sur le germe $g^{x} \in \Gamma_{q}^{r}$. On obtient alors le diagramme suivant

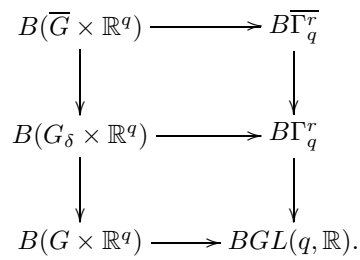

On se spécialise maintenant au cas où $G=\operatorname{Diff} f_{U}^{r}\left(\mathbb{R}^{q}\right)$ est le groupe des $C^{r}$-difféomorphismes de $\mathbb{R}^{q}$ à support dans un disque $U$. L'application

$$
B\left(\bar{G} \times \mathbb{R}^{q}\right) \longrightarrow B \overline{\Gamma_{q}^{r}}
$$

peut s'interpréter de la manière suivante : l'espace $B\left(\bar{G} \times \mathbb{R}^{q}\right)=B \bar{G} \times$ $\mathbb{R}^{q}$ possède un feuilletage de codimension $q$, transverse aux facteurs $\mathbb{R}^{q}$ donc une structure de Haefliger (avec une trivialisation globale de son fibré normal). L'application

$$
B \overline{\operatorname{Diff} f_{U}^{r}\left(\mathbb{R}^{q}\right)} \times \mathbb{R}^{q} \longrightarrow B \overline{\Gamma_{q}^{r}}
$$

passe alors au quotient

$$
\Sigma^{q}\left(B \overline{\operatorname{Diff}_{U}^{r}\left(\mathbb{R}^{q}\right)}\right) \longrightarrow B \overline{\Gamma_{q}^{r}}
$$

où $\Sigma$ est le foncteur de suspension. Par adjonction, on obtient une application

$$
B \overline{\operatorname{Diff} f_{U}^{r}\left(\mathbb{R}^{q}\right)} \longrightarrow \Omega^{q}\left(B \overline{\Gamma_{q}^{r}}\right)
$$

où $\Omega$ est le foncteur de lacets. Voici alors le théorème de Thurston-Mather. 
Théorème 2.3.14 (Thurston-Mather). L'application

$$
B \overline{D i f f_{U}^{r}\left(\mathbb{R}^{q}\right)} \longrightarrow \Omega^{q}\left(B \overline{\Gamma_{q}^{r}}\right)
$$

induit un isomorphisme en homologie intégrale, i.e.

$$
H_{*}\left(B \overline{\operatorname{Diff} f_{U}^{r}\left(\mathbb{R}^{q}\right)}, \mathbb{Z}\right) \cong H_{*}\left(\Omega^{q}\left(B \overline{\Gamma_{q}^{r}}\right), \mathbb{Z}\right) .
$$

Le cas où $q=1$ est dû à Mather ([Mat73]) et le cas général à Thurston. Une rédaction des idées de Thurston se trouve dans [Mat79]. On peut remplacer dans le théorème $\mathbb{R}^{q}$ par une variété quelconque $M$ de dimension $q$ et obtenir un isomorphisme

$$
H_{*}\left(B \overline{\operatorname{Diff} f_{U}^{r}\left(\mathbb{R}^{q}\right)}, \mathbb{Z}\right) \cong H_{*}\left(B \overline{\operatorname{Diff} f_{c}^{r}(M)}, \mathbb{Z}\right)
$$

qui inclut comme cas particulier le théorème 2.2 .15 (avec $*=1$ et $r=$ $\infty)$. Une autre approche est de déduire directement le théorème $2.2 .15 \mathrm{du}$ théorème 2.3.14 (comme cela est fait dans l'appendice de [Mat84]).

Pour conclure, donnons une application du théorème précédent. Rappelons qu'un espace topologique $X$ est dit $n$-connexe si ses groupes d'homotopies jusqu'à l'ordre $n$ sont triviaux. On sait depuis Haefliger (voir [Hae71]) que $B \overline{\Gamma_{q}^{r}}$ est $q$-connexe pour tout $r$, il est alors facile de déduire des théorèmes 2.2.15 et 2.3.14 que $B \overline{\Gamma_{q}^{\infty}}$ est $(q+1)$-connexe. Pour d'autres résultats concernant la topologie des espaces $B \bar{\Gamma}$, on renvoie le lecteur à la jolie présentation de [Tsu].

\subsection{Simplicité de Dif $f_{0}^{r}(M)$ pour $r \neq 3$}

Dans les sections précédentes, on est parvenu à démontrer la simplicité du groupe Dif $f_{0}^{\infty}(M)$ pour une surface compacte $M$. Une étape essentielle de la preuve était le cas particulier $M=\mathbb{T}^{2}$ (le théorème d'Herman-Sergeraert) où l'on a fait usage d'un théorème d'inversion locale adapté aux problèmes de "petits diviseurs". Dans le cas où le degré de différentiabilité $r$ est fini, ces techniques d'analyse ne sont plus applicables et il faut donc d'autres méthodes pour étudier la simplicité du groupe Dif $f_{0}^{r}(M)$ pour $r \in \mathbb{N}^{*}$.

Le but de cette section est de donner quelques idées sur la preuve du théorème suivant, dû à Mather ([Mat74a],[Mat75] et [Mat84], voir [Eps84] et [Ha195] pour plus de détails).

Théorème 2.4.1 (Mather). Soit $M$ une surface compacte. Si $r \neq 3$, alors le groupe Dif $f_{0}^{r}(M)$ est simple.

Remarque 2.4.2. En fait, ce théorème est valable pour une variété compacte $M$ quelconque, sous la restriction $r \neq \operatorname{dim}(M)+1$. La preuve est identique.

Commençons par deux simplifications immédiates. D'une part, au vu du théorème d'Epstein (annexe B), il suffit de prouver que ce groupe est 
parfait. D'autre part, il suffit de traiter le cas où $M=\mathbb{R}^{2}$ : en effet, par le lemme de fragmentation 2.2.1 (et la remarque 2.2.3), tout élément de Dif $f_{0}^{r}(M)$ est un produit de difféomorphismes à support dans des ouverts difféomorphes au plan $\mathbb{R}^{2}$, que l'on peut donc considérer comme des difféomorphismes de $\mathbb{R}^{2}$ à support compact. Notons $D$ if $f_{0, c}^{r}\left(\mathbb{R}^{2}\right)$ le groupe des difféomorphismes du plan compactement $C^{r}$-isotopes à l'identité. La perfection de $\operatorname{Dif} f_{0}^{r}(M)$ est ainsi équivalente à celle de $\operatorname{Dif} f_{0, c}^{r}\left(\mathbb{R}^{2}\right)$, et on peut reformuler le théorème de Mather de la manière suivante.

Théorème 2.4.3 (Mather). Pour $r \neq 3$, le groupe Dif $f_{0, c}^{r}\left(\mathbb{R}^{2}\right)$ est parfait.

La preuve de ce résultat va passer par un contrôle sur la dérivée d'ordre $r$ d'un élément de Dif $f_{0, c}^{r}\left(\mathbb{R}^{2}\right)$. On appelle module de continuité une fonction à valeur réelles $\alpha$, continue et strictement croissante, définie sur un intervalle $[0, \varepsilon]$ pour un certain $\varepsilon>0$ et qui vérifie les propriétés suivantes :

(i) $\alpha(0)=0$;

(ii) $\alpha(t x) \leq t \alpha(x)$, pour $x \in[0, \varepsilon], t \geq 1$ tels que $t x \in[0, \varepsilon]$.

Un exemple classique de module de continuité est donné par la fonction $\alpha(x)=x^{\beta}$ pour $0<\beta \leq 1$. On dit alors qu'une application entre espaces métriques $f: X \rightarrow Y$ est $\alpha$-continue s'il existe des constantes $C, \varepsilon^{\prime}$ avec $C>0$ et $0<\varepsilon^{\prime}<\varepsilon$ telles que pour tous $x$ et $y$ dans $X$,

$$
d(x, y)<\varepsilon^{\prime} \Longrightarrow d(f(x), f(y))<C \alpha(d(x, y)) .
$$

Pour $\alpha(x)=x^{\beta}$, une fonction $\alpha$-continue est une fonction Lipschitz pour $\beta=1$ et Hölder pour $0<\beta<1$. On définit de manière évidente la notion d'application localement $\alpha$-continue, et on peut alors introduire la classe de régularité $C^{r, \alpha}$.

Définition 2.4.4. Soient $U \subseteq \mathbb{R}^{2}$ un ouvert et $f: U \rightarrow \mathbb{R}^{2}$. On dit que $f$ est de classe $C^{r, \alpha}$ si elle est de classe $C^{r}$ et que sa dérivée d'ordre $r$ est localement $\alpha$-continue.

Les applications de classe $C^{r, \alpha}$ disposent de propriétés dont on fera usage dans la suite :

(i) une somme d'applications $C^{r, \alpha}$ est $C^{r, \alpha}$;

(ii) une composée d'applications $C^{r, \alpha}$ est $C^{r, \alpha}$;

(iii) l'inverse de classe $C^{1}$ d'une application $C^{r, \alpha}$ est $C^{r, \alpha}$.

Pour les vérifications de ces propriétés, on peut consulter [Mat74a] ou [Hal95].

Introduisons maintenant quelques quantités (semi-normes) mesurant la taille des applications de classe $C^{r, \alpha}$. Pour une application $f: U \rightarrow \mathbb{R}^{2}$ de classe $C^{r, \alpha}$ avec $\alpha$ défini sur $[0, \varepsilon]$, on pose

$$
\|f\|_{r, \alpha}=\sup \left\{\frac{\left\|D^{r} f(x)-D^{r} f(y)\right\|}{\alpha(\|x-y\|)} \mid x, y \in U, x \neq y,\|x-y\| \leq \varepsilon\right\}
$$


et si $f$ est un $C^{r, \alpha}$-difféomorphisme à support compact, on note

$$
\mu_{r, \alpha}(f)=\|f-1\|_{r, \alpha}
$$

en notant 1 l'application identité. On aura besoin de l'estimation suivante, qui résulte de la formule donnant la dérivée d'ordre $r$ d'une composition.

Lemme 2.4.5. Soient $r \geq 1$ et $\alpha$ un module de continuité. Il existe $\delta>0$ et $C>0$ tels que si $f, g$ sont deux $C^{r, \alpha}$-difféomorphismes à support compact vérifiant $\mu_{r, \alpha}(f)<\delta$ et $\mu_{r, \alpha}(g)<\delta$, alors

$$
\mu_{r, \alpha}(f g) \leq \mu_{r, \alpha}(f)+\mu_{r, \alpha}(g)+C \mu_{r, \alpha}(f) \mu_{r, \alpha}(g) .
$$

Pour un module de continuité $\alpha$, on convient de noter $\operatorname{Dif} f_{0, c}^{r}\left(\mathbb{R}^{2}, \alpha\right)$ l'ensemble des difféomorphismes de classe $C^{r, \alpha}$ qui sont compactement $C^{r, \alpha}$ isotopes à l'identité (i.e. l'isotopie est à support compact et elle est de classe $\left.C^{r, \alpha}\right)$. D'après ce qui précède, l'ensemble $D$ if $f_{0, c}^{r}\left(\mathbb{R}^{2}, \alpha\right)$ a une structure de groupe topologique (pour la topologie $C^{r, \alpha}$ ). En raison de l'égalité

$$
\operatorname{Diff} f_{0, c}^{r}\left(\mathbb{R}^{2}\right)=\bigcup_{\alpha} \operatorname{Diff} f_{0, c}^{r}\left(\mathbb{R}^{2}, \alpha\right)
$$

où l'union est prise sur tous les modules de continuité, il est suffisant d'établir la perfection de $\operatorname{Dif} f_{0, c}^{r}\left(\mathbb{R}^{2}, \alpha\right)$ pour tout module de continuité $\alpha$.

On suppose désormais $r>3$ (le cas où $1 \leq r<3$ est en quelque sorte symétrique et se démontre de manière analogue, voir [Mat75]) et on fixe un module de continuité $\alpha$. Le problème est le suivant : étant donné un élément $f$ appartenant à $D$ if $f_{0, c}^{r}\left(\mathbb{R}^{2}, \alpha\right)$ proche de l'identité, on cherche à résoudre l'équation

$$
f=\left[u_{1}, v_{1}\right] \ldots\left[u_{n}, v_{n}\right]
$$

avec des inconnues $u_{i}, v_{i} \in D i f f_{0, c}^{r}\left(\mathbb{R}^{2}, \alpha\right)$ pour $1 \leq i \leq n$. En notant $[f]$ la classe d'équivalence de $f$ dans l'abélianisé de $D$ if $f_{0, c}^{r}\left(\mathbb{R}^{2}, \alpha\right)$, l'équation se lit plus simplement

$$
[f]=0 .
$$

On va expliquer comment Mather parvient à résoudre cette équation par un argument astucieux ayant recours au théorème de point fixe de LeraySchauder.

La construction fondamentale est la suivante. On se fixe un réel $A>$ 1 (qui sera appelé à être grand) et on introduit une suite croissante de rectangles (voir la figure 2.6)

$$
D_{2}=[-2,2]^{2} \subseteq D_{1}=[-2,2] \times[-2 A, 2 A] \subseteq D_{0}=[-2 A, 2 A]^{2} .
$$

Pour $i=1,2$, il existe une application

$$
\Psi_{i}: U_{i} \longrightarrow \text { Diff } f_{0, c}^{1}\left(\mathbb{R}^{2}\right)
$$




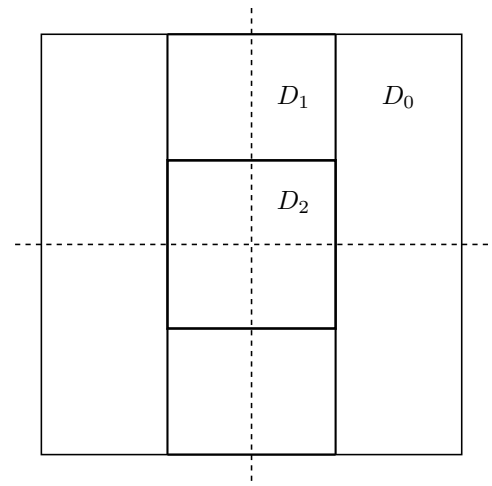

FIG. $2.6-D_{2} \subseteq D_{1} \subseteq D_{0}$

où

$$
U_{i}=\left\{u \in \operatorname{Diff} f_{0, c}^{1}\left(\mathbb{R}^{2}\right) \mid \operatorname{supp}(u) \subseteq D_{i-1}\right\}
$$

est un $C^{1}$-voisinage de l'identité dans l'ensemble des $C^{1}$-difféomorphismes de $\mathbb{R}^{2}$ à support dans $D_{i-1}$, et telle que $\Psi_{i}\left(U_{i}\right)$ soit inclus dans $U_{i+1}$. Cette application $\Psi_{i}$ vérifie les propriétés suivantes, pour tout $u$ appartenant à $U_{i}$ :

(1) $\Psi_{i}(1)=1$;

(2) si $u$ est de classe $C^{r, \alpha}$, il en est de même pour $\Psi_{i}(u)$;

(3) l'application $\Psi_{i}$ restreinte aux $C^{r}$-difféomorphismes est continue;

(4) si $u$ est de classe $C^{r, \alpha}$, alors $[u]=\left[\Psi_{i}(u)\right]$ dans $D$ if $f_{0, c}^{r}\left(\mathbb{R}^{2}, \alpha\right)$;

(5) il existe $\delta>0, C>0$ tels que si $u$ est de classe $C^{r, \alpha}$, alors

$$
\mu_{r, \alpha}(u)<\delta \Longrightarrow \mu_{r, \alpha}\left(\Psi_{i}(u)\right) \leq C A \mu_{r, \alpha}(u)
$$

Remarquons que par les propriétés (1) et (3), quitte à rétrécir $U_{i}$, on peut supposer que $u$ est isotope à l'identité par une isotopie à support dans l'intérieur de $D_{i-1}$ et que $\Psi_{i}(u)$ est isotope à l'identité par une isotopie à support dans l'intérieur de $D_{i}$. En utilisant ceci ainsi que la propriété (2), on obtient que si $u$ est de classe $C^{r, \alpha}, u$ et $\Psi_{i}(u)$ sont dans $D$ if $f_{0, c}^{r}\left(\mathbb{R}^{2}, \alpha\right)$, ce qui nous assure que la propriété (4) a bien un sens. La propriété (4) et l'estimation (5) seront cruciales.

Admettons pour le moment l'existence de ces applications $\Psi_{i}$ et de leurs propriétés et montrons dans ce paragraphe comment le théorème $2.4 .3 \mathrm{~s}$ 'en déduit. On note (abusivement) $A$ l'application de $\mathbb{R}^{2}$ qui est la multiplication par le réel $A$. Prenons $f$ dans $D$ if $f_{0, c}^{r}\left(\mathbb{R}^{2}, \alpha\right)$ proche de l'identité 
et à support dans l'intérieur de $D_{2}$. Pour $u$ appartenant à $D$ if $f_{0, c}^{r}\left(\mathbb{R}^{2}, \alpha\right)$ également proche de l'identité et à support dans l'intérieur de $D_{2}$, on définit le difféomorphisme

$$
\tilde{u}=\Psi_{2} \Psi_{1}\left(A f u A^{-1}\right) .
$$

Puisque $\left[A f u A^{-1}\right]=[f u]$ dans $D$ if $f_{0, c}^{r}\left(\mathbb{R}^{2}, \alpha\right)$ (on peut facilement construire $\tilde{A} \in \operatorname{Diff} f_{0, c}^{r}\left(\mathbb{R}^{2}, \alpha\right)$ tel que $\left.\tilde{A}_{\mid D_{2}}=A\right)$, on obtient

$$
[\tilde{u}]=[f u]
$$

par la propriété (4). Ainsi, si l'on parvient à trouver un difféomorphisme $u$ tel que $\tilde{u}=u$, on obtient le résultat escompté, à savoir $[f]=0$. On note alors $R_{A, f}(u)=\tilde{u}$, et l'on part ainsi à la recherche d'un point fixe, dans un espace convenable, pour $R_{A, f}$. Pour $\varepsilon>0$, on définit la boule

$$
B_{\varepsilon}=\left\{u \in \operatorname{Diff} f_{0, c}^{r}\left(\mathbb{R}^{2}, \alpha\right) \mid \operatorname{supp}(u) \subseteq \operatorname{int}\left(D_{2}\right), \mu_{r, \alpha}(u) \leq \varepsilon\right\} .
$$

Il n'est pas difficile de vérifier que, pour la $C^{r}$-topologie, $B_{\varepsilon}$ est un compact convexe. Par le théorème de point fixe de Leray-Schauder, toute application continue de $B_{\varepsilon}$ dans lui-même possède un point fixe. On définit alors

$$
R_{A, f}: B_{\varepsilon} \longrightarrow \text { Diff } f_{0, c}^{r}\left(\mathbb{R}^{2}, \alpha\right) .
$$

Par la propriété (3), $R_{A, f}$ est continue. Il suffit donc d'établir le lemme suivant.

Lemme 2.4.6. Avec les notations précédentes, il existe un réel $A$ et $\varepsilon>0$ tels que si $\mu_{r, \alpha}(f) \leq \varepsilon$, alors

$$
R_{A, f}\left(B_{\varepsilon}\right) \subseteq B_{\varepsilon} .
$$

Démonstration. Soit $u \in B_{\varepsilon}$, par le lemme 2.4.5, on obtient

$$
\mu_{r, \alpha}(f u) \leq 3 \varepsilon
$$

pour $\varepsilon$ suffisamment petit. De l'inégalité

$$
\mu_{r, \alpha}\left(A f u A^{-1}\right) \leq A^{1-r} \mu_{r, \alpha}(f u)
$$

il vient

$$
\mu_{r, \alpha}\left(A f u A^{-1}\right) \leq 3 A^{1-r} \varepsilon .
$$

On fixe maintenant $\varepsilon$ suffisamment petit pour que l'on puisse appliquer la propriété (5) à $A f u A^{-1}$ et $\Psi_{1}\left(A f u A^{-1}\right)$ et obtenir

$$
\mu_{r, \alpha}\left(R_{A, f}(u)\right) \leq 3 C^{2} A^{1-r+2} \varepsilon
$$

où $C$ est la constante (indépendante de $A$ ) apparaissant dans l'estimation (5). On a $1-r+2<0$ par hypothèse (c'est ici qu'intervient la condition 
sur le degré $r$ ), donc en fixant $A$ suffisamment grand, on a $3 C^{2} A^{1-r+2}<1$ ce qui donne

$$
\mu_{r, \alpha}\left(R_{A, f}(u)\right) \leq \varepsilon .
$$

De plus, on vérifie facilement que $R_{A, f}(u)$ est à support dans l'intérieur de $D_{2}$, ce qui conclut la preuve du lemme.

D'après la discussion précédente, il nous reste donc à construire notre application $\Psi_{i}$ et de vérifier les propriétés de (1) à (5). Dans la suite, on ne précise plus que $i=1,2$ pour ne pas alourdir le texte. Cette construction étant longue et parfois technique, on va seulement en donner une idée et l'on renvoie à [Mat74a] ou [Hal95] pour des arguments détaillés.

(a) Construction de transformations auxiliaires.

Dans ce paragraphe, on construit quelques transformations qui vont nous être utile pour établir un critère de conjugaison. On se donne $\rho$ une fonction lisse à valeurs réelles, à support dans $[-2 A-1,2 A+1]$ et qui vaut identiquement 1 sur $[-2 A, 2 A]$. Par abus, on note encore $\rho$ la fonction sur $\mathbb{R}^{2}$ définie par

$$
\rho\left(x_{1}, x_{2}\right)=\rho\left(x_{1}\right) \rho\left(x_{2}\right)
$$

et qui est à support dans $[-2 A-1,2 A+1]^{2}$. On note $\partial_{i}$ le $i$-ème champ de vecteurs coordonnées, et l'on définit

$$
\tau_{i}=\exp \left(\rho \partial_{i}\right)
$$

i.e. $\tau_{i}$ est le temps 1 du flot de $\rho \partial_{i}$. Notons que $\tau_{i}$ est un difféomorphisme à support dans $[-2 A-1,2 A+1]^{2}$. On définit également une application $\varphi_{i}$, uniquement déterminée par les conditions suivantes :

(i) son domaine de définition est

$$
\operatorname{Dom}\left(\varphi_{i}\right)=\left\{x \in \mathbb{R}^{2}|| x_{j} \mid \leq 2 A+1, j \neq i\right\} ;
$$

(ii) $\left(\varphi_{i}\right)_{*}\left(\partial_{i}\right)=\rho \partial_{i}$;

(iii) $\varphi_{i \mid D_{0}}=1$.

L'application $\varphi_{i}$ est ainsi un difféomorphisme sur son image, elle préserve les droites dont la coordonée $x_{j}, j \neq i$, est constante et "contracte" la bande $\operatorname{Dom}\left(\varphi_{i}\right)$ sur le carré $[-2 A-1,2 A+1]^{2}$ (voir la figure 2.7). Une manière équivalente de définir $\varphi_{i}$ est de prendre $\left(\psi_{i}^{t}\right)_{t \in \mathbb{R}}$ le flot de $\rho \partial_{i}$ et de poser

$$
\varphi_{1}\left(x_{1}, x_{2}\right)=\psi_{1}^{x_{1}}\left(0, x_{2}\right) \quad ; \quad \varphi_{2}\left(x_{1}, x_{2}\right)=\psi_{2}^{x_{2}}\left(x_{1}, 0\right) .
$$

Enfin, notons $T_{i}$ la translation unitaire dans la $i$-ème direction. Ainsi

$$
T_{i}=\exp \left(\partial_{i}\right)
$$

et puisque $\tau_{i}=\exp \left(\rho \partial_{i}\right)$ et $\left(\varphi_{i}\right)_{*}\left(\partial_{i}\right)=\rho \partial_{i}$, il est clair que $\varphi_{i}$ conjugue $T_{i}$ à $\tau_{i}$, i.e. $\tau_{i}=\varphi_{i} T_{i}\left(\varphi_{i}\right)^{-1}$. 


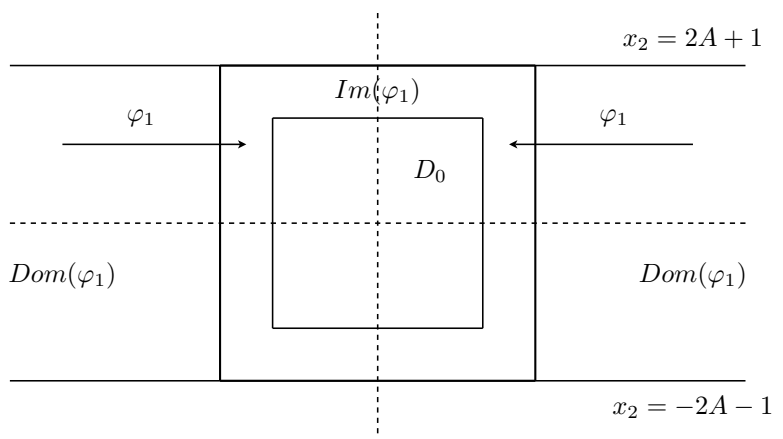

FIG. 2.7 - Action de $\varphi_{1}$

(b) Un lemme de conjugaison.

Soient $u$ et $v$ des $C^{r, \alpha}$-difféomorphismes, tous deux à support dans $D_{0}$ et $C^{1}$-proches de l'identité. On donne dans ce paragraphe une condition suffisante pour que les difféomorphismes $\tau_{i} u$ et $\tau_{i} v$ soient conjugués dans Dif $f_{0, c}^{r}\left(\mathbb{R}^{2}, \alpha\right)$, par un procédé d' "enroulement" de $u$ et $v$.

On introduit les cylindres $C_{1}=\mathbb{T} \times \mathbb{R}$ et $C_{2}=\mathbb{R} \times \mathbb{T}$ et $\pi_{i}: \mathbb{R}^{2} \rightarrow C_{i}$ la projection canonique. On note $p_{i}: \mathbb{R}^{2} \rightarrow \mathbb{R}$ l'application qui oublie la $i$-ème coordonnée et, de manière abusive, on note également $p_{i}: C_{i} \rightarrow \mathbb{R}$. On définit alors une application

$$
\Gamma_{i}(u): C_{i} \longrightarrow C_{i}
$$

de la manière suivante. Soient $\zeta$ appartenant à $C_{i}$ et $x$ un élément de $\mathbb{R}^{2}$ qui relève $\zeta$ (i.e. $\pi_{i}(x)=\zeta$ ) et qui vérifie $x_{i}<-2 A$. On se donne $N$ suffisamment grand pour que

$$
\left(\left(T_{i} u\right)^{N}(x)\right)_{i}>2 A .
$$

On pose alors

$$
\Gamma_{i}(u)(\zeta)=\pi_{i}\left(\left(T_{i} u\right)^{N}(x)\right)
$$

On vérifie sans peine que $\Gamma_{i}(u)$ ne dépend pas du choix de $N$ et de $x$ puisque $u$ est l'identité à l'extérieur de $D_{0}$. En faisant une construction similaire, on montre que $\Gamma_{i}(u)$ est inversible et que c'est donc un difféomorphisme de classe $C^{r, \alpha}$ (au sens où ses relevés à $\mathbb{R}^{2}$ sont des difféomorphismes de classe $\left.C^{r, \alpha}\right)$. Remarquons que $\Gamma_{i}(1)=1$ et si $\zeta$ vérifie $\left|\zeta_{j}\right|<2 A$ pour $j$ différent de $i$, alors $\Gamma_{i}(u)(\zeta)=\zeta$. L'application $\Gamma_{i}$ est définie sur un ensemble de difféomorphismes, à support dans $D_{0}$, et $C^{1}$-proches de l'identité dans $D$ if $f_{0, c}^{r}\left(\mathbb{R}^{2}, \alpha\right)$ et elle est à valeurs dans l'espace $D$ if $f_{0, c}^{r}\left(C_{i}, \alpha\right)$ : pour la $C^{r}$-topologie sur l'espace de départ et l'espace d'arrivée, $\Gamma_{i}$ est continue. 
De plus, on peut estimer explicitement $\mu_{r, \alpha}\left(\Gamma_{i}(u)\right)$ en fonction de $\mu_{r, \alpha}(u)$ à l'aide du lemme 2.4.5 (voir [Eps84] ou [Hal95] pour ces vérifications).

On a une action évidente de $\mathbb{T}$ sur notre cylindre $C_{i}$. On note $G_{i}$ le groupe des difféomorphismes de $C_{i}$, de classe $C^{r, \alpha}$ et équivariant sous l'action de $\mathbb{T}$. Voici le lemme de conjugaison de Mather.

Lemme 2.4.7 (Mather). Soient $u$ et $v$ des $C^{r, \alpha}$-difféomorphismes à support dans $D_{0}$ et $C^{1}$-proches de l'identité. Si $\Gamma_{i}(v) \Gamma_{i}(u)^{-1}$ appartient à $G_{i}$, alors les difféomorphismes $\tau_{i} u$ et $\tau_{i} v$ sont conjugués dans Dif $f_{0, c}^{r}\left(\mathbb{R}^{2}, \alpha\right)$. En particulier, on a

$$
[u]=[v] \in H_{1}\left(\operatorname{Diff} f_{0, c}^{r}\left(\mathbb{R}^{2}, \alpha\right)\right) .
$$

La preuve de ce lemme est technique, on va seulement donner l'idée et on renvoie aux références pour les détails. Il n'est pas difficile de voir que pour $N$ suffisamment grand, l'application

$$
\Lambda_{i}(x)=\left(T_{i} v\right)^{N}\left(T_{i} u\right)^{-N}(x)
$$

définit un difféomorphisme de $\mathbb{R}^{2}$ de classe $C^{r, \alpha}$ qui conjugue $T_{i} u$ et $T_{i} v$. Maintenant, on dispose d'un difféomorphisme

$$
\varphi_{i}: \operatorname{Dom}\left(\varphi_{i}\right) \longrightarrow[-2 A-1,2 A+1]^{2}
$$

qui conjugue $T_{i}$ et $\tau_{i}$. Ceci implique que $\varphi_{i} \Lambda_{i} \varphi_{i}^{-1}$ conjugue $\tau_{i} u$ à $\tau_{i} v$ mais seulement sur son domaine de définition, à savoir $[-2 A-1,2 A+1]^{2}$. Le problème (technique) est donc de prolonger $\varphi_{i} \Lambda_{i} \varphi_{i}^{-1}$ en un difféomorphisme de $\mathbb{R}^{2}$ de classe $C^{r, \alpha}$, et c'est ici qu'intervient l'hypothèse essentielle que $\Gamma_{i}(v) \Gamma_{i}(u)^{-1}$ commute à l'action de $\mathbb{T}$.

(c) Construction des applications $\Psi_{i}$.

Passons maintenant à la construction des applications $\Psi_{i}$. Rappelons que pour un élément $u$ à support dans l'intérieur de $D_{i-1}$, de classe $C^{1}$ et $C^{1}$-proche de l'identité, on souhaite définir $v=\Psi_{i}(u)$ à support dans l'intérieur de $D_{i}$, de classe $C^{1}$ et $C^{1}$-proche de l'identité.

Notons $\theta_{i}$ la coordonnée angulaire de $C_{i}$. Si $u$ est suffisamment petit, il existe un unique difféomorphisme $h_{i}$ de $C_{i}$ tel que $h_{i}$ soit l'identité sur $\left\{\theta_{i}=\right.$ $0\}$ et qui vérifie $h_{i} \Gamma_{i}^{-1}(u) \in G_{i}$. De manière équivalente, $g_{i}=h_{i} \Gamma_{i}^{-1}(u)$ est l'unique élément de $G_{i}$ qui coïncide avec $\Gamma_{i}(u)$ sur $\left\{\theta_{i}=0\right\}$, on peut alors donner une formule explicite, par exemple

$$
g_{1}(\theta, r)=\Gamma_{1}(u)(0, r)+(\theta, 0) \quad ; \quad g_{2}(r, \theta)=\Gamma_{1}(u)(r, 0)+(0, \theta) .
$$

De plus, $h_{i}$ est proche de l'identité car c'est le cas pour $\Gamma_{i}(u)$. En utilisant une fonction plateau, on peut décomposer $h_{i}=h_{i}^{1} h_{i}^{0}$ avec $h_{i}^{1}, h_{i}^{0}$ des difféomorphismes de $C_{i}$ tels que $h_{i}^{1}$ soit l'identité au voisinage de $\left\{\theta_{i}=0\right\}$ 
et $h_{i}^{0}$ soit l'identité au voisinage de $\left\{\theta_{i}=1 / 2\right\}$. Introduisons maintenant les ensembles

$$
E_{i}^{-}=\left\{x \in \mathbb{R}^{2} \mid-3 / 2<x_{i}<-1 / 2\right\} \quad ; \quad E_{i}^{+}=\left\{x \in \mathbb{R}^{2} \mid 0<x_{i}<1\right\}
$$

et définissons un difféomorphisme $v$ de $\mathbb{R}^{2}$ de la manière suivante : $v$ est l'identité sur $\mathbb{R}^{2} \backslash\left(E_{i}^{-} \cup E_{i}^{+}\right)$, il est semi-conjugué à $h_{i}^{0} \operatorname{sur} E_{i}^{-}$, i.e.

$$
\left(\pi_{i} v\right)_{\mid E_{i}^{-}}=\left(h_{i}^{0} \pi_{i}\right)_{\mid E_{i}^{-}}
$$

et il est semi-conjugué à $h_{i}^{1} \operatorname{sur} E_{i}^{+}$, i.e.

$$
\left(\pi_{i} v\right)_{\mid E_{i}^{+}}=\left(h_{i}^{1} \pi_{i}\right)_{\mid E_{i}^{+}} .
$$

On pose finalement $\Psi_{i}(u)=v$. Le difféomorphisme $u$ étant à support dans $D_{i-1}$, il est clair que $\Psi_{i}(u)$ est à support dans $D_{i}$. Par construction, on a

$$
\Gamma_{i}(v)=h_{i}^{1} h_{i}^{0}=h_{i}
$$

de telle sorte que $\Gamma_{i}(v) \Gamma_{i}^{-1}(u)$ appartienne à $G_{i}$. Les difféomorphismes $\tau_{i} u$ et $\tau_{i} v$ sont donc conjugués dans $\operatorname{Dif} f_{0, c}^{r}\left(\mathbb{R}^{2}, \alpha\right)$ par le lemme 2.4.7, ce qui permet de conclure que

$$
[u]=\left[\Psi_{i}(u)\right]=[v] \in H_{1}\left(\operatorname{Diff}_{0, c}^{r}\left(\mathbb{R}^{2}, \alpha\right)\right) .
$$

On obtient ainsi la propriété (4). Les propriétés (1) et (2) sont évidentes par construction. La propriété (3) provient du fait que l'application $\Gamma_{i}$ est continue pour les $C^{r}$-topologies. Enfin la propriété (5) résulte essentiellement de l'estimation de $\mu_{r, \alpha}\left(\Gamma_{i}(u)\right)$ en fonction de $\mu_{r, \alpha}(u)$. Ceci termine l'esquisse de la preuve du théorème de Mather 2.4.3.

Terminons ce chapitre par quelques remarques. Comme il a déjà été mentionné, l'argument précédent montre de manière plus générale que le groupe des $C^{r}$-difféomorphismes d'une variété compacte $M$ de dimension $n$ est parfait si $r \neq n+1$. En termes d'espaces classifiants de feuilletages (voir la section précédente), on obtient le corollaire suivant.

Corollaire 2.4.8. L'espace $B \overline{\Gamma_{n}^{r}}$ est $(n+1)$-connexe pour $r \neq n+1$.

Pour $r=+\infty$, ce schéma de démonstration a été adapté par Epstein ([Eps84]) pour redémontrer la simplicité du groupe $\operatorname{Dif} f_{0}^{\infty}(M)$ pour une variété compacte $M$.

Enfin pour $r=n+1$, la question reste totalement ouverte. Pour illustrer la subtilité de ce problème, mentionnons un très joli exemple dû à Mather ([Mat85]). En notant $G$ le groupe des $C^{1}$-difféomorphismes de $\mathbb{R}$ à support compact et dont la dérivée est à variation borné, on peut construire de manière simple un morphisme de groupe surjectif

$$
\pi: G \longrightarrow \mathbb{R}
$$

et en déduire que ce groupe $G$ n'est pas parfait. En revanche, la restriction de ce morphisme au groupe des $C^{2}$-difféomorphismes de $\mathbb{R}$ à support compact est identiquement nulle. 


\section{Chapitre 3}

\section{Difféomorphismes conservatifs}

Soit $M$ une surface compacte munie d'une forme volume $\omega$, i.e. d'une 2-forme différentielle lisse sur $M$ partout non nulle, que l'on suppose normalisée par $\int_{M} \omega=1$. Une application lisse $g: M \rightarrow M$ préserve l'aire si on a $g^{*} \omega=\omega$, ce qui revient à demander que son jacobien soit constant égal à 1. On note alors $\operatorname{Diff} f^{\infty}(M, \omega)$ le groupe des difféomorphismes préservant l'aire, et $\operatorname{Diff} f^{\infty}(M, \partial M, \omega)$ son analogue dans le cas à bord.

Contrairement au cas non conservatif, on va montrer que la composante connexe de l'identité de ces groupes n'est pas simple en général. En effet, sous l'hypothèse de non-nullité du premier groupe d'homologie de $M$, on sait construire un morphisme de groupe non trivial de $\operatorname{Dif} f_{0}^{\infty}(M, \omega)$ vers $\mathbb{R}$. Donnons un exemple simple d'un tel invariant sur le tore $\mathbb{T}^{2}$.

Soit $\varphi \in \operatorname{Diff} f_{0}^{\infty}\left(\mathbb{T}^{2}, \omega\right)$, où $\omega$ est une forme volume quelconque (on pourrait en fait choisir la forme volume canonique sans perte de généralité). On voit $\mathbb{T}^{2}$ comme $\mathbb{R}^{2} / \mathbb{Z}^{2}$ et on relève $\varphi$ en un difféomorphisme du plan $\tilde{\varphi}$ en relevant une isotopie de $\varphi$ à l'identité. Ainsi $\tilde{\varphi}$ commute à l'action de $\pi_{1}\left(\mathbb{T}^{2}\right) \cong \mathbb{Z}^{2}$ sur $\mathbb{R}^{2}$, donc si on se fixe un point $x \in \mathbb{T}^{2}$ et un relevé $\tilde{x}$ de $x$, le vecteur $\tilde{\varphi}(\tilde{x})-\tilde{x} \in \mathbb{R}^{2}$ ne dépend que de $x$ (et pas de $\tilde{x}$ ). Il mesure le déplacement de $x$ sous l'isotopie, i.e. le nombre de tours sur le tore, dans les directions verticales et horizontales, qu'effectue $x$ sous l'action de l'isotopie. Si $\mu$ désigne la mesure de probabilité associée à $\omega$ (i.e. $\left.\mu(A)=\int_{A} \omega\right)$, on définit alors le déplacement moyen de l'isotopie par le vecteur

$$
\int_{\mathbb{T}^{2}}(\tilde{\varphi}(\tilde{x})-\tilde{x}) d \mu(x) .
$$

Quitte à considérer ce nombre dans $\mathbb{T}^{2}$, il ne dépend plus que de $\varphi$ et $\mu$. On le note $\rho(\varphi, \mu)$ : c'est le vecteur de rotation (ou de translation) de $\varphi$ 
associé à $\mu$. L'invariance de $\mu$ entraîne facilement que l'application

$$
\begin{array}{ccc}
\rho: \operatorname{Diff}_{0}^{\infty}\left(\mathbb{T}^{2}, \omega\right) & \longrightarrow \mathbb{T}^{2} \\
\varphi & \longmapsto \rho(\varphi, \mu)
\end{array}
$$

est un morphisme de groupes et en particulier que $\rho(\varphi, \mu)$ est un invariant de conjugaison. Ce morphisme est évidemment surjectif : la rotation $R_{\alpha}$ de vecteur $\alpha \in \mathbb{T}^{2}$ a bien sûr un vecteur de rotation $\alpha$. Son noyau définit ainsi un sous-groupe normal propre de $\operatorname{Diff} f_{0}^{\infty}\left(\mathbb{T}^{2}, \omega\right)$.

Dans ce chapitre, on va commencer par généraliser cette construction à une surface compacte $M$ quelconque, et ce de diverses manières sous le nom générique de premier invariant de Calabi. Ceci établira la nonsimplicité de $D$ if $f_{0}^{\infty}(M, \omega)$ pour toutes les surfaces mises à part la sphère et le disque. On étudiera ensuite le noyau de ce morphisme, qui coïncide avec le groupe des difféomorphismes hamiltoniens. Si $M$ est sans bord, on expliquera un théorème de Banyaga qui énonce que ce noyau est simple. Si $M$ est à bord, on construira un second invariant de Calabi sur le groupe des difféomorphismes hamiltoniens ; le théorème de Banyaga affirme encore une fois que le noyau de ce second invariant est simple.

\subsection{Premier invariant de Calabi}

Pour simplifier, on va commencer par le cas où notre surface $M$ est fermée. On peut naturellement se demander si le choix de $\omega$ a une quelconque influence sur les propriétés algébriques de $\operatorname{Dif} f_{0}^{\infty}(M, \omega)$. La réponse est donnée par le théorème classique suivant ([Mos65]).

Théorème 3.1.1 (Moser). Soient $\omega_{0}, \omega_{1}$ deux formes volumes sur $M$ de masse totale 1 . Alors il existe un élément $\varphi$ dans Dif $f_{0}^{\infty}(M)$ tel que $\varphi^{*} \omega_{1}=\omega_{0}$.

En particulier, les propriétés algébriques de $\operatorname{Dif} f_{0}^{\infty}(M, \omega)$ ne dépendent pas du choix de $\omega$ car deux formes volumes distinctes nous donnent des sous-groupes conjugués dans Dif $f_{0}^{\infty}(M)$.

Démonstration. Voici une preuve "à la Moser". Considérons le chemin de formes volumes $\omega_{t}=t \omega_{1}+(1-t) \omega_{0}$ (l'ensemble des formes volumes est convexe). Il suffit de trouver une isotopie $\left(\varphi^{t}\right)_{t \in[0,1]}$ dans Dif $f_{0}^{\infty}(M)$ qui vérifie, pour tout $t$ appartenant à $[0,1]$,

$$
\varphi^{t *} \omega_{t}=\omega_{0}
$$

car son temps 1 est une solution à notre problème. Puisque $\varphi^{0 *} \omega_{0}=\omega_{0}$, on est donc ramené à l'équation

$$
\frac{d}{d t}\left(\varphi^{t *} \omega_{t}\right)=0
$$


Remarquons que pour résoudre cette équation, il suffit de trouver un champ de vecteurs $X_{t}$ (dépendant du temps) tel que

$$
\mathcal{L}_{X_{t}} \omega_{t}+\frac{d}{d t} \omega_{t}=0
$$

car par compacité de $M, X_{t}$ s'intégrerait en l'isotopie recherchée. D'une part, puisque $\omega_{t}$ est fermée pour tout $t$ dans [0,1], la formule de Lie-Cartan nous donne $\mathcal{L}_{X_{t}} \omega_{t}=d i_{X_{t}} \omega_{t}$. D'autre part,

$$
\frac{d}{d t} \omega_{t}=\omega_{1}-\omega_{0}
$$

est cohomologue à zéro car $\int_{M} \omega_{0}=\int_{M} \omega_{1}$, elle s'écrit donc $d \alpha$ pour une 1 -forme $\alpha$. Tout revient alors à résoudre l'équation

$$
i_{X_{t}} \omega_{t}+\alpha=0
$$

qui possède évidemment une solution $X_{t}$ par non-dégénérescence de $\omega_{t}$.

Passons maintenant à la construction d'un morphisme non trivial sur le groupe $\operatorname{Dif} f_{0}^{\infty}(M, \omega)$, qu'on appelle "premier invariant de Calabi" ([Cal70]). Il y a plusieurs constructions possibles, avec des interprétations différentes selon que l'on considère ce morphisme à valeurs dans le premier groupe d'homologie ou de cohomologie. Dans tous les cas, il est plus commode de commencer par définir ce morphisme sur les isotopies.

On note $\widetilde{\operatorname{Diff} f_{0}^{\infty}}(M, \omega)$ le revêtement universel de $\operatorname{Dif} f_{0}^{\infty}(M, \omega)$. Par le théorème de Moser, Dif $f_{0}^{\infty}(M)$ agit transitivement sur l'espace $V(M)$ des formes volumes normalisées, avec un sous-groupe d'isotropie qui est par définition $\operatorname{Dif} f_{0}^{\infty}(M, \omega)$. L'espace $V(M)$ étant convexe, on déduit de la suite exacte d'homotopie associée à la fibration

$$
\text { Diff } f_{0}^{\infty}(M, \omega) \longrightarrow \text { Diff } f_{0}^{\infty}(M) \longrightarrow V(M)
$$

que la première inclusion est une équivalence d'homotopie. En particulier, puisque $\operatorname{Dif} f_{0}^{\infty}(M)$ est localement contractile, il en est de même pour le groupe Dif $f_{0}^{\infty}(M, \omega)$. Ainsi, un élément de $\widetilde{\operatorname{Diff} f_{0}^{\infty}}(M, \omega)$ est une classe d'homotopie (à extrémités fixes) de chemins

$$
t \in[0,1] \longmapsto \varphi^{t} \in \text { Diff } f_{0}^{\infty}(M, \omega)
$$

avec $\varphi^{0}=1$.

Prenons donc $\Phi=\left(\varphi^{t}\right)_{t \in[0,1]}$ une isotopie dans le groupe $\operatorname{Diff} f_{0}^{\infty}(M, \omega)$ et soit $X_{t}$ son champ de vecteurs tangent, i.e. le champ défini pour tout $t$ dans $[0,1]$ par

$$
\frac{d}{d t} \varphi^{t}=X_{t} \varphi^{t}
$$

Puisque $\Phi$ préserve l'aire, la divergence de $X_{t}$ est nulle donc la 1-forme $i_{X_{t}} \omega$ est fermée pour tout $t$ appartenant à $[0,1]$. 
Définition 3.1.2. Le flux d'une isotopie $\Phi$ dans Dif $f_{0}^{\infty}(M, \omega)$ est défini par la classe de cohomologie

$$
\operatorname{Flux}(\Phi)=\int_{0}^{1}\left[i_{X_{t}} \omega\right] d t \in H^{1}(M, \mathbb{R}) .
$$

En réalité, le flux de $\Phi$ ne dépend que de la classe d'homotopie de $\Phi$ (à extrémités fixes). Pour montrer cela, on va donner une interprétation géométrique du morphisme de flux (voir la figure 3.1). En utilisant l'identification

$$
H^{1}(M, \mathbb{R}) \cong \operatorname{Hom}\left(H_{1}(M, \mathbb{R}), \mathbb{R}\right)
$$

regardons l'action de $\operatorname{Flux}(\Phi)$ sur $[\gamma] \in H_{1}(M, \mathbb{R})$.

Lemme 3.1.3. Le nombre Flux $(\Phi) .[\gamma]$ représente l'aire algébrique balayée par le lacet $\gamma$ sous l'isotopie $\Phi$. En particulier, il ne dépend que de la classe d'homotopie de $\Phi$.

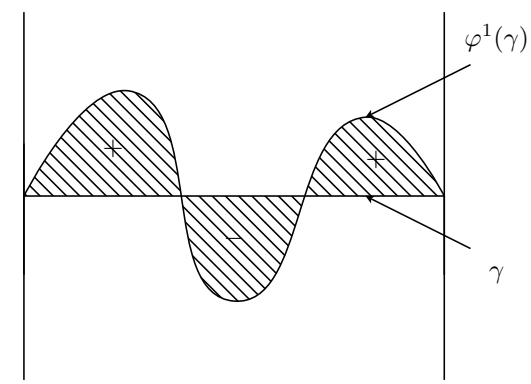

FIG. 3.1 - Interprétation géométrique de $F l u x(\Phi) \cdot \gamma$

Démonstration. On calcule

$$
\begin{aligned}
\operatorname{Flux}(\Phi) \cdot[\gamma] & =\int_{0}^{1} \int_{\gamma} i_{X_{t}} \omega d t \\
& =\int_{0}^{1} \int_{0}^{1} \omega\left(X_{t}(\gamma(s)), \dot{\gamma}(s)\right) d t d s \\
& =\int_{[0,1] \times \mathbb{T}}(\Phi \gamma)^{*} \omega
\end{aligned}
$$

où $\Phi \gamma:[0,1] \times \mathbb{T} \rightarrow M$ est définie par $\Phi \gamma(t, \theta)=\varphi^{t}(\gamma(\theta))$. Le flux ne dépend que de la classe d'homologie de $\gamma$, puisque si $\gamma^{\prime}$ est homologue à $\gamma$, 
le théorème de Stokes et le fait que $i_{X_{t}} \omega$ soit fermée pour tout $t$ implique que

$$
\int_{\gamma} i_{X_{t}} \omega=\int_{\gamma^{\prime}} i_{X_{t}} \omega
$$

La quantité

$$
\operatorname{Flux}(\Phi) \cdot[\gamma]=\int_{[0,1] \times \mathbb{T}}(\Phi \gamma)^{*} \omega
$$

s'interprète bien comme l'aire algébrique du "cylindre singulier" bordé par $\gamma$ et $\varphi^{1}(\gamma)$ et balayé par $\Phi$.

Donnons-nous maintenant $\Phi^{\prime}$ une isotopie homotope (à extrémités fixes) à $\Phi$. Il s'en suit que $\left(\Phi^{\prime} \gamma\right)^{*} \omega$ est cohomologue à $(\Phi \gamma)^{*} \omega$, et donc par le théorème de Stokes, Flux $\left(\Phi^{\prime}\right) \cdot[\gamma]-F l u x(\Phi) \cdot[\gamma]$ est une intégrale sur le bord de $[0,1] \times \mathbb{T}$, qui s'annule car l'homotopie entre $\Phi^{\prime}$ et $\Phi$ est à extrémités fixes. Ceci prouve que $\operatorname{Flux}(\Phi) \cdot[\gamma]$ ne dépend que de $[\Phi]$.

Proposition 3.1.4. Le flux définit un morphisme de groupe surjectif

$$
\begin{aligned}
\text { Flux : } \widetilde{\operatorname{Diff}_{0}^{\infty}}(M, \omega) & \longrightarrow H^{1}(M, \mathbb{R}) \\
{[\Phi] } & \longmapsto \int_{0}^{1}\left[i_{X_{t}} \omega\right] d t .
\end{aligned}
$$

Démonstration. Le lemme précédent nous assure que

$$
\text { Flux : } \widetilde{\operatorname{Diff} f_{0}^{\infty}}(M, \omega) \longrightarrow H^{1}(M, \mathbb{R})
$$

est une application bien définie.

On vérifie facilement que c'est un morphisme de groupes : il suffit de considérer la loi de groupe dans $\widetilde{\operatorname{Diff} f_{0}^{\infty}}(M, \omega)$ comme la concaténation des chemins puis d'utiliser la linéarité de l'intégrale pour obtenir

$$
\operatorname{Flux}(\Phi * \Psi) \cdot[\gamma]=\operatorname{Flux}(\Psi) \cdot[\gamma]+\operatorname{Flux}(\Phi) \cdot\left[\psi^{1}(\gamma)\right]
$$

et on conclut en utilisant le fait que $\left[\psi^{1}(\gamma)\right]=[\gamma]$. Enfin, si $\alpha \in H^{1}(M, \mathbb{R})$, la non-dégénérescence de $\omega$ nous donne un unique champ de vecteurs autonome $X$ tel que $i_{X} \omega=\alpha$, son flot $\left(\phi^{t}\right)_{t \in[0,1]}$ vérifie alors Flux $\left(\left(\phi^{t}\right)_{t \in[0,1]}\right)=$ $\alpha$, ce qui démontre la surjectivité.

On définit le groupe de flux par

$$
\Gamma_{\omega}=\operatorname{Flux}\left(\pi_{1}\left(\operatorname{Diff}_{0}^{\infty}(M, \omega)\right)\right) \subseteq H^{1}(M, \mathbb{R}) .
$$

En notant

$$
P_{\omega}=\left\{\int_{C} \omega \mid C \in H^{2}(M, \mathbb{Z})\right\} \subseteq \mathbb{R}
$$

le groupe des périodes de $\omega$, le groupe de flux $\Gamma_{\omega}$ est en fait un sous-groupe de $H^{1}\left(M, P_{\omega}\right)$. En particulier, $\Gamma_{\omega}$ est toujours dénombrable. On obtient ainsi un morphisme surjectif

$$
\text { Flux }: \text { Diff } f_{0}^{\infty}(M, \omega) \longrightarrow H^{1}(M, \mathbb{R}) / \Gamma_{\omega}
$$


que l'on appelle encore morphisme de flux (et que l'on note abusivement de la même manière). Voyons ce que devient ce morphisme sur des exemples.

Exemple 3.1.5 (La sphère $\mathbb{S}^{2}$ ). Son premier groupe de cohomologie est trivial donc il en est de même du morphisme de flux.

Exemple 3.1.6 (La surface $\Sigma_{g}, g>1$ ). La situation est maintenant plus intéressante. Dans ce cas, le morphisme est non trivial mais

$$
\Gamma_{\omega}=\operatorname{Flux}\left(\pi_{1}\left(\operatorname{Diff} f_{0}^{\infty}\left(\Sigma_{g}, \omega\right)\right)\right)
$$

est trivial car Dif $f_{0}^{\infty}\left(\Sigma_{g}, \omega\right)$ est contractile. Voici une petite explication de ce fait. Par une remarque précédente, il suffit de prouver que Dif $f_{0}^{\infty}\left(\Sigma_{g}\right)$ est contractile, car il a le même type d'homotopie que Dif $f_{0}^{\infty}\left(\Sigma_{g}, \omega\right)$. Pour cela, on considère $M\left(\Sigma_{g}\right)$ l'espace des structures (presque) complexes sur $\Sigma_{g}$. On fait alors agir Diff $f_{0}^{\infty}\left(\Sigma_{g}\right)$ sur $M\left(\Sigma_{g}\right)$, et on montre que cette action est propre et libre et nous donne une fibration au dessus du quotient

$$
M\left(\Sigma_{g}\right) / \operatorname{Diff} f_{0}^{\infty}\left(\Sigma_{g}\right)
$$

qui s'identifie naturellement à l'espace de Teichmüller de $\Sigma_{g}$. Ce dernier est contractile (car homéomorphe à $\mathbb{R}^{6 g-6}$ via les coordonnées de FenchelNielsen). L'espace total $M\left(\Sigma_{g}\right)$ étant contractile, la suite exacte d'homotopie associée à la fibration nous donne la contractibilité de Dif $f_{0}^{\infty}\left(\Sigma_{g}\right)$ (pour plus de détails, voir [EE69]). Au final, on obtient

$$
\text { Flux : Diff } f_{0}^{\infty}\left(\Sigma_{g}, \omega\right) \longrightarrow H^{1}\left(\Sigma_{g}, \mathbb{R}\right) \cong \mathbb{R}^{2 g} .
$$

Exemple 3.1.7 (Le tore $\mathbb{T}^{2}$ ). Cette fois ci, on trouve

$$
\pi_{1}\left(\text { Dif } f_{0}^{\infty}\left(\mathbb{T}^{2}, \omega\right)\right) \cong \mathbb{Z}^{2}
$$

avec des méthodes analogues aux précédentes. On a alors $\Gamma_{\omega} \cong H^{1}\left(\mathbb{T}^{2}, \mathbb{Z}\right)$ et on obtient donc

$$
\text { Flux : Diff } f_{0}^{\infty}\left(\mathbb{T}^{2}, \omega\right) \longrightarrow H^{1}\left(\mathbb{T}^{2}, \mathbb{R}\right) / H^{1}\left(\mathbb{T}^{2}, \mathbb{Z}\right) \cong \mathbb{T}^{2} .
$$

On peut même donner une formule explicite dans ce cas (voir [MS98] pour le calcul). Soient $\Phi=\left(\varphi^{t}\right)_{t \in[0,1]}$ un élément de Dif $\left.\widetilde{f_{0}^{\infty}\left(\mathbb{T}^{2}\right.}, \omega\right)$, et $\widetilde{\Phi}=$ $\left(\tilde{\varphi}^{t}\right)_{t \in[0,1]}$ son relèvement à $\mathbb{R}^{2}$ tel que $\tilde{\varphi}^{0}$ soit l'identité. Alors en notant

$$
\left(a_{1}, a_{2}\right)=J \int_{\mathbb{T}^{2}}\left(\tilde{\varphi}^{1}(\tilde{x})-\tilde{x}\right) d \mu(x) \in \mathbb{R}^{2}
$$

où $J$ est la structure complexe canonique de $\mathbb{R}^{2}$, on trouve

$$
\operatorname{Flux}(\Phi)=a_{1} d x_{1}+a_{2} d x_{2} \in H^{1}\left(\mathbb{T}^{2}, \mathbb{R}\right) .
$$


Le flux est donc nul si et seulement si

$$
\int_{\mathbb{T}^{2}}\left(\tilde{\varphi}^{1}(\tilde{x})-\tilde{x}\right) d \mu(x)=0
$$

ce qui signifie géométriquement que $\varphi$ (ou plus exactement $\tilde{\varphi}^{1}$ ) préserve le centre de masse.

Remarque 3.1.8. Le groupe de flux $\Gamma_{\omega}$ est toujours dénombrable. Une question, connue sous le nom de la conjecture du flux et qui fût longtemps un problème ouvert, était de savoir si $\Gamma_{\omega}$ est un sous-groupe discret de $H^{1}\left(M, P_{\omega}\right)$ (pour la topologie induite). On vient de voir que c'est toujours le cas pour les surfaces fermées (c'est aussi vrai pour les surfaces ouvertes car alors le groupe est trivial, voir plus loin), la question est donc intéressante pour les variétés symplectiques $(M, \omega)$ en dimension plus grande. On peut montrer sans trop de difficulté que la réponse est positive si $\omega$ est à périodes entières (i.e. $P_{\omega}$ est discret, comme pour le tore $\mathbb{T}^{2 n}$ muni de sa forme symplectique canonique) ou si $\omega$ provient d'une forme de Kähler (comme pour $\mathbb{C} P^{n}$ muni de la forme de Fubini-Study).

Cette question est importante car elle équivaut à la fermeture du groupe $\operatorname{Ham}(M, \omega)$ dans $\operatorname{Symp}(M, \omega)$ pour la topologie $C^{1}$ (voir la prochaine section pour les définitions). D'autres cas particuliers ont été résolus, mais ce n'est que très récemment que Ono ([Ono06]) a démontré le résultat dans toute sa généralité pour une variété fermée.

Donnons maintenant une autre construction, équivalente au morphisme de flux, à valeurs cette fois dans le $H_{1}(M, \mathbb{R})$ (voir [Ghy03] par exemple). C'est la notion de cycle asymptotique de Schwartzman (ou de vecteur de rotation, par analogie avec le cas du tore).

Pour $\Phi$ un élément de $\widetilde{\operatorname{Diff} f_{0}^{\infty}}(M, \omega)$, on définit $S \operatorname{ch}(\Phi) \in H_{1}(M, \mathbb{R})$ par son action sur les classes de cohomologie de 1-formes différentielles fermées. Prenons $x$ dans $M$, et notons $\gamma_{\Phi, x}$ le chemin de $x$ à $\varphi(x)$ obtenu en suivant l'isotopie. Soit $[\alpha] \in H^{1}(M, \mathbb{R})$ une classe de cohomologie, on peut alors intégrer l'un de ses représentants le long de la courbe $\gamma_{\Phi, x}$ pour obtenir une fonction de $x$ puis faire la moyenne sur $M$. On pose

$$
\operatorname{Sch}(\Phi) \cdot \alpha=\int_{M}\left(\int_{\gamma_{\Phi, x}} \alpha\right) d \mu(x) \in \mathbb{R} .
$$

Si $\alpha$ est exacte, on vérifie sans peine que $S c h(\Phi) \cdot \alpha=0$ (essentiellement parce que $\varphi$ préserve la mesure) donc $S c h(\Phi)$ est bien défini sur le $H^{1}(M, \mathbb{R})$. La linéarité de l'intégrale nous donne aussitôt que $S \operatorname{ch}(\Phi)$ est une forme linéaire, donc $S \operatorname{ch}(\Phi)$ est un élément de $H_{1}(M, \mathbb{R})$.

Comme pour le morphisme de flux, cette expression ne dépend que de la classe d'homotopie à extrémités fixes de $\Phi$, donc on a une application

$$
\text { Sch }: \widetilde{\operatorname{Diff}_{0}^{\infty}}(M, \omega) \longrightarrow H_{1}(M, \mathbb{R}) \text {. }
$$


De plus, si $\psi$ est le temps 1 d'une isotopie $\Psi \in \widetilde{\operatorname{Diff} f_{0}^{\infty}}(M, \omega)$, on a

$$
\begin{aligned}
\operatorname{Sch}(\Phi * \Psi) \cdot \alpha & =\int_{M}\left(\int_{\gamma_{\Phi * \Psi, x}} \alpha\right) d \mu(x) \\
& =\int_{M}\left(\int_{\gamma_{\Psi, x}} \alpha\right) d \mu(x)+\int_{M}\left(\int_{\gamma_{\Phi, \psi(x)}} \alpha\right) d \mu(x) \\
& =\int_{M}\left(\int_{\gamma_{\Psi, x}} \alpha\right) d \mu(x)+\int_{M}\left(\int_{\gamma_{\Phi, x}} \alpha\right) d \mu(x) \\
& =S \operatorname{ch}(\Psi) \cdot \alpha+\operatorname{Sch}(\Phi) \cdot \alpha .
\end{aligned}
$$

Tout ceci nous assure que l'invariant de Schwartzman définit bien un morphisme de groupes. Le lien avec le morphisme de flux s'obtient par dualité de Poincaré :

$$
\begin{aligned}
\int_{M} \operatorname{Flux}(\Phi) \wedge \alpha & =\int_{M}\left(\int_{0}^{1} i_{X_{t}} \omega d t\right) \wedge \alpha \\
& =\int_{M} \int_{0}^{1} i_{X_{t}} \omega \wedge \alpha d t \\
& =\int_{M} \int_{0}^{1} i_{X_{t}} \alpha \wedge \omega d t \\
& =\int_{M}\left(\int_{0}^{1} i_{X_{t}} \alpha(x) d t\right) d \mu(x) \\
& =\int_{M}\left(\int_{\gamma_{\Phi, x}} \alpha\right) d \mu(x)
\end{aligned}
$$

ce qui nous donne bien

$$
\int_{M} \operatorname{Flux}(\Phi) \wedge \alpha=\operatorname{Sch}(\Phi) \cdot \alpha .
$$

Comme pour le morphisme de flux, on définit

$$
\text { Sch }: \operatorname{Diff} f_{0}^{\infty}(M, \omega) \longrightarrow H_{1}(M, \mathbb{R}) / \Gamma_{\omega}^{\prime}
$$

où $\Gamma_{\omega}^{\prime}=\operatorname{Sch}\left(\pi_{1}\left(\operatorname{Diff} f_{0}^{\infty}(M, \omega)\right)\right)$.

Exemple 3.1.9 (Le tore $\left.\mathbb{T}^{2}\right)$. Prenons $\varphi$ un élément de Diff $f_{0}^{\infty}\left(\mathbb{T}^{2}, \omega\right)$, temps 1 d'une isotopie $\Phi$. Soient $\tilde{\varphi}$ un relevé de $\varphi$ obtenu en relevant l'isotopie, et $\tilde{x}$ un relevé de $x$. On souhaite vérifier directement que $S \operatorname{ch}(\varphi)$ nous donne le vecteur de rotation. Pour cela, soit $d x_{1}, d x_{2}$ la base canonique de $H^{1}\left(\mathbb{T}^{2}, \mathbb{R}\right)$, on a alors

$$
\left(\int_{\gamma_{\Phi, x}} d x_{1}, \int_{\gamma_{\Phi, x}} d x_{2}\right)=\tilde{\varphi}(\tilde{x})-\tilde{x} \in \mathbb{R}^{2}
$$


ce qui nous donne Sch $(\Phi)=\int_{M}(\tilde{\varphi}(\tilde{x})-\tilde{x}) d \mu(x)$, et donc en éliminant la dépendance vis-à-vis du choix de l'isotopie $\Phi$ (et donc de $\tilde{\varphi})$, on a bien

$$
\operatorname{Sch}(\varphi)=\int_{M}(\tilde{\varphi}(\tilde{x})-\tilde{x}) d \mu(x) \in \mathbb{T}^{2} .
$$

Donnons enfin une dernière approche plus "dynamique" de cet invariant, proche de l'idée originale de Schwartzman (voir [Sch57]). Revenons un instant à notre exemple introductif, à savoir le vecteur de rotation d'un difféomorphisme du tore isotope à l'identité. Soient $\varphi \in \operatorname{Diff} f_{0}^{\infty}\left(\mathbb{T}^{2}, \omega\right)$ et $\tilde{\varphi}$ un relevé de $\varphi$. On se donne un point $x$ dans $\mathbb{T}^{2}$ et un relevé $\tilde{x}$ dans $\mathbb{R}^{2}$. On peut alors lui associer un vecteur de rotation $\rho(\tilde{\varphi}, \tilde{x})$ appartenant à $\mathbb{R}^{2}$ si la limite

$$
\rho(\tilde{\varphi}, \tilde{x})=\lim _{n \rightarrow+\infty} \frac{1}{n}\left(\tilde{\varphi}^{n}(\tilde{x})-\tilde{x}\right)
$$

existe. Un autre choix pour $\tilde{\varphi}$ ou $\tilde{x}$ nous donne la même limite modulo $\mathbb{Z}^{2}$, on peut donc définir un vecteur $\rho(\varphi, x) \in \mathbb{T}^{2}$. Une simple application du théorème ergodique de Birkhoff nous assure que $\rho(\varphi, x)$ existe pour $\mu$-presque tout $x$ et que sa valeur moyenne est

$$
\int_{\mathbb{T}^{2}} \rho(\varphi, x) d \mu(x)=\rho(\varphi, \mu) .
$$

C'est cette version "asymptotique" que l'on va généraliser aux surfaces quelconques. On reprend les notations précédentes.

Commençons par étendre notre isotopie $\Phi=\left(\varphi^{t}\right)_{t \in[0,1]}$ pour des temps $t \in \mathbb{R}$ par la formule

$$
\varphi^{t+1}=\varphi^{t} \varphi^{1}
$$

Notons $\gamma_{n}(x)$ le segment d'orbite entre $x$ et $\varphi^{n}(x)$ pour un entier $n$, et $\tilde{\gamma}_{n}(x)$ le lacet obtenu en fermant le chemin $\gamma_{n}(x)$ par une géodésique minimale entre $x$ et $\varphi^{n}(x)$ (on a seulement besoin de joindre deux points quelconques par une courbe de longueur uniformément bornée).

Alors $\left[\tilde{\gamma}_{n}(x)\right] \in H_{1}(M, \mathbb{R})$, et la limite

$$
\rho(\Phi, x)=\lim _{n \rightarrow+\infty} \frac{1}{n}\left[\tilde{\gamma}_{n}(x)\right]
$$

si elle existe, représente le "déplacement homologique moyen" de $x$ sous l'isotopie. Son action sur les classes de cohomologie $[\alpha] \in H^{1}(M, \mathbb{R})$ est donnée par

$$
\rho(\Phi, x) \cdot \alpha=\lim _{n \rightarrow+\infty} \frac{1}{n} \int_{\tilde{\gamma}_{n}(x)} \alpha .
$$

Nous allons montrer que cette limite existe pour $\mu$-presque tout $x$ et qu'en notant

$$
\rho(\Phi, \mu)=\int_{M} \rho(\Phi, x) d \mu(x)=\int_{M}\left(\lim _{n \rightarrow+\infty} \frac{1}{n}\left[\tilde{\gamma}_{n}(x)\right]\right) d \mu(x)
$$


on a l'égalité

$$
\operatorname{Sch}(\Phi)=\rho(\Phi, \mu) \in H_{1}(M, \mathbb{R}) .
$$

Pour vérifier cette relation, étudions l'action de $\rho(\Phi, \mu)$ sur $[\alpha] \in H^{1}(M, \mathbb{R})$.

On veut donner un sens à l'expression suivante :

$$
\rho(\Phi, \mu) \cdot \alpha=\int_{M}\left(\lim _{n \rightarrow+\infty} \frac{1}{n} \int_{\tilde{\gamma}_{n}(x)} \alpha\right) d \mu(x) .
$$

Or

$$
\int_{\tilde{\gamma}_{n}(x)} \alpha=\int_{\gamma_{n}(x)} \alpha+\int_{c} \alpha
$$

où $c$ est une géodésique minimale entre $x$ et $\varphi^{n}(x)$, donc $\left|\int_{c} \alpha\right|<K$ avec une constante $K$ qui ne dépend ni de $x$, ni de $n$. L'expression

$$
\frac{1}{n} \int_{\gamma_{n}(x)} \alpha=\frac{1}{n} \sum_{k=0}^{n-1}\left(\int_{\gamma_{1}\left(\varphi^{k}(x)\right)} \alpha\right)=\frac{1}{n} \sum_{k=0}^{n-1} f\left(\varphi^{k}(x)\right)
$$

est une somme de Birkhoff pour la fonction $f(x)=\int_{\gamma_{1}(x)} \alpha$, ce qui prouve la convergence de la suite

$$
\frac{1}{n} \int_{\gamma_{n}(x)} \alpha
$$

pour $\mu$-presque tout point $x$. Puisque

$$
\left|\frac{1}{n} \int_{\tilde{\gamma}_{n}(x)} \alpha-\frac{1}{n} \int_{\gamma_{n}(x)} \alpha\right| \leq \frac{K}{n}
$$

la suite

$$
\frac{1}{n} \int_{\tilde{\gamma}_{n}(x)} \alpha
$$

converge également $\mu$-presque partout et vers la même limite. De plus, le théorème de Birkhoff nous donne la moyenne de cette limite

$$
\rho(\Phi, \mu) \cdot \alpha=\int_{M}\left(\lim _{n \rightarrow+\infty} \frac{1}{n} \int_{\tilde{\gamma}_{n}(x)} \alpha\right) d \mu(x)=\int_{M}\left(\int_{\gamma_{1}(x)} \alpha\right) d \mu(x) .
$$

Ceci montre bien que $S c h(\Phi)=\rho(\Phi, \mu)$, puisque le chemin $\gamma_{1}(x)$ est exactement ce qu'on avait appelé $\gamma_{\Phi, x}$ précédemment.

Remarque 3.1.10. Dans le dernier chapitre, on donnera encore une autre construction de ce morphisme, valable dans un cadre purement topologique. On peut d'ores et déjà remarquer que l'on peut définir $\rho(\Phi, \mu)$ pour une mesure borélienne invariante $\mu$ arbitraire, et pas seulement pour une mesure provenant d'une forme volume. 
Toutes les constructions précédentes restent valables pour une surface à bord $M$, sous la restriction que l'isotopie fixe un voisinage du bord. On peut même donner une formule plus simple, car dans ce cas la forme symplectique est exacte, i.e. $\omega=d \lambda$ (puisque le second groupe de cohomologie de la surface est trivial). En effet, si $\varphi \in D$ if $f_{0}^{\infty}(M, \partial M, \omega)$ est le temps 1 d'une isotopie $\Phi=\left(\varphi^{t}\right)_{t \in[0,1]}$ fixant un voisinage du bord, on a

$$
\left[i_{X_{t}} \omega\right]=\frac{d}{d t}\left[\varphi^{t *} \lambda\right]
$$

et donc

$$
\operatorname{Flux}(\Phi)=\left[\varphi^{*} \lambda-\lambda\right]
$$

ne dépend que $\varphi$. On obtient ainsi des morphismes non triviaux

$$
\text { Flux :Diff } f_{0}^{\infty}(M, \partial M, \omega) \longrightarrow H^{1}(M, \partial M, \mathbb{R})
$$

et par dualité

$$
\operatorname{Sch}: \operatorname{Diff} f_{0}^{\infty}(M, \partial M, \omega) \longrightarrow H_{1}(M, \partial M, \mathbb{R}) .
$$

Lorsque $M$ est une surface à bord, la restriction pour les isotopies au voisinage $\mathrm{du}$ bord permet de se ramener à la surface ouverte sans bord $M^{\prime}=M \backslash \partial M$ et aux isotopies à support compact dans $M^{\prime}$. Les morphismes précédents sont alors à valeurs dans le premier groupe de cohomologie (ou d'homologie) à support compact de $M^{\prime}$.

On en déduit facilement que pour une surface compacte, si son premier groupe de cohomologie est non trivial (i.e. si $M$ n'est pas la sphère ou le disque), les groupes $\operatorname{Dif} f_{0}^{\infty}(M, \omega)$ et $\operatorname{Diff} f_{0}^{\infty}(M, \partial M, \omega)$ ne sont pas simples, le noyau du premier invariant de Calabi étant un sous-groupe normal non trivial. La question suivante est donc d'examiner de plus près ce noyau.

\subsection{Difféomorphismes hamiltoniens}

Pour simplifier la situation, commençons encore par le cas où $M$ est fermée. Dans cette section, on va commencer par introduire un sous-groupe normal de $\operatorname{Diff} f_{0}^{\infty}(M, \omega)$ naturellement lié au premier invariant de Calabi, et l'on va ensuite démontrer qu'il coïncide avec le noyau de ce morphisme.

Soit $\varphi$ appartenant à Dif $f_{0}^{\infty}(M, \omega)$, alors par définition il existe une isotopie préservant le volume

$$
t \in[0,1] \longmapsto \varphi^{t} \in \operatorname{Diff}^{\infty}(M, \omega)
$$

telle que $\varphi^{0}=1$ et $\varphi^{1}=\varphi$. Si $X_{t}$ désigne le champ de vecteurs tangent à $\left(\varphi^{t}\right)_{t \in[0,1]}$, on sait que la 1 -forme $i_{X_{t}} \omega$ est fermée pour tout $t$ dans $[0,1]$. 
Puisque $\omega$ n'est rien d'autre qu'une forme symplectique, il est naturel de se demander si $X_{t}$ est un gradient symplectique, i.e. si $i_{X_{t}} \omega$ est exacte pour tout t dans $[0,1]$.

Définition 3.2.1. On dit qu'une isotopie est hamiltonienne si la 1-forme $i_{X_{t}} \omega$ est exacte pour tout $t \in[0,1]$. Un difféomorphisme $\varphi \in$ Dif $f_{0}^{\infty}(M, \omega)$ est hamiltonien s'il existe une isotopie hamiltonienne $\Phi=\left(\varphi^{t}\right)_{t \in[0,1]}$ avec $\varphi^{1}=\varphi$.

Fixons quelques notations. Pour $t$ appartenant à $[0,1]$, on désigne par $H_{t}$ l'unique primitive de la forme $i_{X_{t}} \omega$ vérifiant $\int_{M} H_{t} \omega=0$. On dit que la fonction

$$
\begin{aligned}
& H \quad: \quad[0,1] \times M \quad \longrightarrow \quad \mathbb{R} \\
& (t, x) \quad \longmapsto \quad H(t, x)=H_{t}(x)
\end{aligned}
$$

est le hamiltonien normalisé qui engendre l'isotopie hamiltonienne $\Phi_{H}=$ $\left(\varphi_{H}^{t}\right)_{t \in[0,1]}$ et le difféomorphisme hamiltonien $\varphi=\varphi_{H}^{1}$. On note $\mathcal{A}$ le sousespace vectoriel de $C^{\infty}(M)$ constitué des fonctions de moyenne nulle, et

$$
\mathcal{H}=\left\{H \in C^{\infty}([0,1] \times M) \mid \forall t \in[0,1], H_{t} \in \mathcal{A}\right\}
$$

l'espace des hamiltoniens normalisés.

Remarquons que les isotopies hamiltoniennes sont essentiellement insensibles au reparamétrage. En effet, soit $a:[0,1] \rightarrow \mathbb{R}$ une fonction croissante lisse telle que $a(0)=0$. Si l'on définit

$$
H^{a}(t, x)=a^{\prime}(t) H(a(t), x)
$$

alors

$$
\varphi_{H}^{a(t)}=\varphi_{H^{a}}^{t}
$$

et $\Phi_{H^{a}}$ est une isotopie hamiltonienne sur le segment $[0, a(1)]$ de hamiltonien normalisé $H^{a}$. Ceci a pour conséquence la possibilité, très utile, de pouvoir représenter les difféomorphismes hamiltoniens par des hamiltoniens définis sur $\mathbb{R} \times M$ et périodique en temps. En effet, si $\varphi$ est un difféomorphisme hamiltonien défini par l'isotopie $\Phi_{H}$, alors en la reparamétrant par une fonction $a:[0,1] \rightarrow[0,1]$ qui vaut 0 au voisinage de 0 et 1 au voisinage de 1 , on obtient une isotopie $\Phi_{H^{a}}$ que l'on peut étendre à $\mathbb{R}$ par la formule

$$
\varphi_{H^{a}}^{t+1}=\varphi_{H^{a}}^{t} \varphi_{H^{a}}^{1} .
$$

Cette dernière égalité équivaut à dire que le hamiltonien normalisé $H_{a}$ est défini sur $\mathbb{T} \times M$, avec $\mathbb{T}=\mathbb{R} / \mathbb{Z}$.

Notons $\operatorname{Ham}(M, \omega)$ l'ensemble des difféomorphismes hamiltoniens, que l'on munit de la topologie $C^{1}$. Par définition, les éléments de $\operatorname{Ham}(M, \omega)$ ont un flux nul : en effet, si $\varphi$ est un difféomorphisme hamiltonien temps 
1 d'une isotopie $\Phi_{H}$, il est alors immédiat que $\left[\Phi_{H}\right]$ est dans le noyau du morphisme de flux

$$
\text { Flux }: \widetilde{\operatorname{Diff}_{0}^{\infty}}(M, \omega) \longrightarrow H^{1}(M, \mathbb{R})
$$

car $\left[d H_{t}\right]=0$ pour tout $t \in[0,1]$. On montrera dans la suite que les difféomorphismes hamiltoniens sont en fait les éléments de $\operatorname{Dif} f_{0}^{\infty}(M, \omega)$ de flux nul.

Avant cela, vérifions que c'est effectivement un sous-groupe normal du groupe Dif $f_{0}^{\infty}(M, \omega)$, ce qui n'est pas clair puisque les difféomorphismes hamiltoniens ne sont pas définis à priori par la préservation d'une structure géométrique.

Proposition 3.2.2. L'ensemble $\operatorname{Ham}(M, \omega)$ est un sous-groupe normal de Dif $f_{0}^{\infty}(M, \omega)$, connexe par arcs.

Démonstration. Pour $\Phi_{H}$ et $\Psi_{K}$ des isotopies hamiltoniennes engendrées par les fonctions $H, K$ appartenant à $\mathcal{H}$, et $\phi$ dans $D$ if $f_{0}^{\infty}(M, \omega)$, on introduit les fonctions suivantes :

$$
\begin{aligned}
& (H \# K)(t, x)=H(t, x)+K\left(t,\left(\varphi_{H}^{t}\right)^{-1}(x)\right) ; \\
& (\bar{H})(t, x)=-H\left(t, \varphi_{H}^{t}(x)\right) ; \\
& \left(\phi^{*} H\right)(t, x)=H(t, \phi(x)) .
\end{aligned}
$$

Les fonctions $H \# K, \bar{H}$ et $\phi^{*} H$ sont clairement dans $\mathcal{H}$. On a alors

$$
\begin{aligned}
& \frac{d}{d t}\left(\varphi_{H}^{t} \psi_{K}^{t}\right)=\dot{\varphi}_{H}^{t} \psi_{K}^{t}+\left(d \varphi_{H}^{t} \psi_{K}^{t}\right) \cdot \dot{\psi}_{K}^{t} \\
& =X_{H} \varphi_{H}^{t} \psi_{K}^{t}+\left(d \varphi_{H}^{t} \psi_{K}^{t}\right) \cdot\left(X_{K} \psi_{K}^{t}\right) \\
& =X_{H} \varphi_{H}^{t} \psi_{K}^{t}+\left(d \varphi_{H}^{t}\left(\varphi_{H}^{t}\right)^{-1} \varphi_{H}^{t} \psi_{K}^{t}\right) \cdot\left(X_{K}\left(\varphi_{H}^{t}\right)^{-1} \varphi_{H}^{t} \psi_{K}^{t}\right)
\end{aligned}
$$

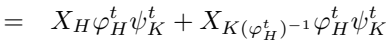

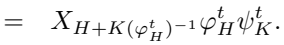

On montre par ce calcul que $H \# K$ engendre l'isotopie

$$
\Phi_{H} \cdot \Psi_{K}: t \in[0,1] \longmapsto \varphi_{H}^{t} \psi_{K}^{t} \in \operatorname{Ham}(M, \omega) .
$$

En utilisant ce résultat, on prouve également que $\bar{H}$ engendre

$$
t \in[0,1] \longmapsto\left(\varphi_{H}^{t}\right)^{-1} \in \operatorname{Ham}(M, \omega)
$$

et enfin il est immédiat de vérifier que $\phi^{*} H$ engendre

$$
t \in[0,1] \longmapsto \phi \varphi_{H}^{t} \phi^{-1} \in \operatorname{Ham}(M, \omega) .
$$

En prenant le temps 1 de ces isotopies, on s'assure que $\operatorname{Ham}(M, \omega)$ est un sous-groupe normal. Pour la connexité par arcs, si $\Phi_{H}$ est une isotopie hamiltonienne avec $\varphi=\varphi_{H}^{1}$, on vérifie facilement que chaque $\varphi_{H}^{s}$ pour $s \in] 0,1[$ est aussi un difféomorphisme hamiltonien pour le reparamétrage $H^{a}$ donné par la fonction $a(t)=s t$. Ceci termine la preuve. 
Remarque 3.2.3. Rappelons qu'un hamiltonien $H$ est dit autonome s'il ne dépend pas du temps. L'isotopie qu'il engendre est alors un flot (ou un sous-groupe à un paramètre) dans le sens où

$$
\varphi_{H}^{t+t^{\prime}}=\varphi_{H}^{t} \varphi_{H}^{t^{\prime}}
$$

et on a la propriété importante de "préservation de l'énergie" : la fonction $H$ est constante le long des orbites du flot. Le temps 1 de ce flot nous donne un exemple pratique de difféomorphisme hamiltonien. Bien que plus maniable, cette notion souffre cependant d'un défaut majeur en ce qui nous concerne : lorsque que l'on compose le temps 1 de deux flots hamiltoniens, on n'obtient pas le temps 1 d'un flot hamiltonien (en bref, il n'y pas de structure de groupe). On est donc contraint de considérer des hamiltoniens non autonomes.

Il est souvent bien utile de voir Dif $f^{\infty}(M)$ (resp. Dif $\left.f^{\infty}(M, \omega)\right)$ comme un groupe de Lie de dimension infinie, son algèbre de Lie s'identifiant à l'algèbre des champs de vecteurs (resp. des champs de vecteurs à divergence nulle) munie du crochet de Lie entre champs de vecteurs. Regardons ce qui se passe pour le groupe $\operatorname{Ham}(M, \omega)$. Si l'on poursuit l'analogie, l'algèbre de Lie de $\operatorname{Ham}(M, \omega)$ est donc l'algèbre des champs de vecteurs $X$ tels que

$$
X(x)=\left.\frac{d}{d t}\right|_{t=0}\left(\varphi^{t}(x)\right)
$$

où $\left(\varphi^{t}\right)_{t \in[0,1]}$ est un chemin dans $\operatorname{Ham}(M, \omega)$ avec $\varphi^{0}=1$. Un tel chemin est une isotopie hamiltonienne (ce point est admis pour le moment, on le montrera dans la section 3.4) donc il est uniquement déterminé par son hamiltonien normalisé, une fonction $H \in \mathcal{H}$. Sous ces conditions, on vérifie que le champ de vecteurs $X$ n'est rien d'autre que le gradient symplectique de $H_{0} \in \mathcal{A}$. L'algèbre de Lie s'identifie donc avec l'espace de fonctions

$$
\mathcal{A}=\left\{H \in C^{\infty}(M) \mid \int_{M} H \omega=0\right\} .
$$

En continuant ainsi, on constate que le crochet de Lie s'assimile au crochet de Poisson dans $\mathcal{A}$ et il n'est pas difficile de s'assurer que l'action adjointe de $\operatorname{Ham}(M, \omega)$ sur $\mathcal{A}$ est l'action classique (à droite) des difféomorphismes sur les fonctions, à savoir $H \mapsto H \varphi$.

Dans le cas où notre surface est à bord, on définit de manière analogue le groupe $\operatorname{Ham}(M, \partial M, \omega)$ en normalisant le hamiltonien $H$ de manière à ce que la fonction $H_{t}=H(t,$.$) soit nulle sur un voisinage du bord indépendant$ de $t$ (en particulier, l'isotopie engendrée fixe un voisinage du bord). Comme on l'a déjà expliqué, cela revient à considérer la surface sans bord $M \backslash \partial M$ et on demande alors à l'isotopie d'être à support compact (dans ce cas, on normalise le hamiltonien pour qu'il soit nul hors de ce compact). 
Donnons maintenant quelques exemples classiques de difféomorphismes hamiltoniens. Comme on va le voir, les exemples les plus simples sont donnés par des hamiltoniens autonomes qui possèdent des propriétés de "symétries".

Exemple 3.2.4 (La sphère $\mathbb{S}^{2}$, voir [Pol01]). Le groupe $H^{1}\left(\mathbb{S}^{2}, \mathbb{R}\right)$ est trivial, on a donc l'égalité

$$
\operatorname{Ham}\left(\mathbb{S}^{2}, \omega\right)=\operatorname{Diff} f_{0}^{\infty}\left(\mathbb{S}^{2}, \omega\right)
$$

où $\omega$ désigne la forme volume canonique de $\mathbb{S}^{2}$. Le groupe $S O(3)$ agit par rotations sur $\mathbb{S}^{2}$, donc il se plonge dans $\operatorname{Ham}\left(\mathbb{S}^{2}, \omega\right)$ (voir la figure 3.2). On peut ainsi remarquer que l'action d'une rotation $A=\exp (a)$ avec a dans so(3) est donnée (de manière infinitésimale) par l'action du gradient symplectique de la fonction hauteur $H_{a}(x)=\langle a, x\rangle$ après identification so $(3) \cong \mathbb{R}^{3}$. On peut généraliser cet exemple en considérant des rotations "fibrées", qui sont définies par des hamiltoniens fonctions uniquement de la hauteur, préservant donc chaque cercle $\left\{H_{a}=r\right\}$ sur lesquels la dynamique est une rotation d'angle $\rho(r)$.

$\mathbb{S}^{2}$

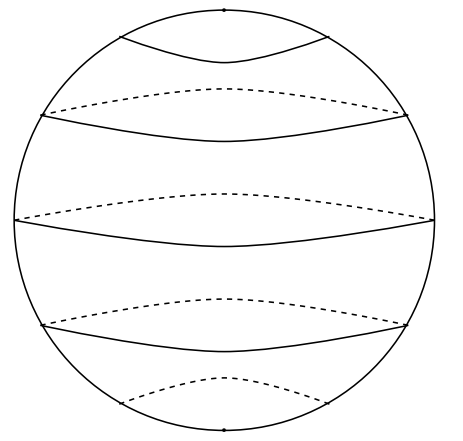

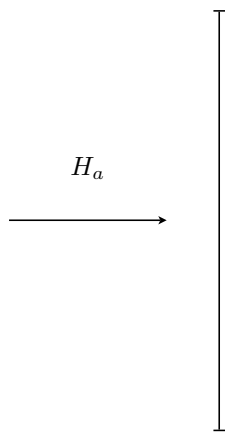

FIG. 3.2 - Exemples de difféomorphismes hamiltoniens de $\mathbb{S}^{2}(a=(0,0,1))$

Exemple 3.2.5 (Le disque $\left.\mathbb{D}^{2}\right)$. On a également

$$
\operatorname{Ham}\left(\mathbb{D}^{2}, \partial \mathbb{D}^{2}, \omega\right)=\operatorname{Diff}_{0}^{\infty}\left(\mathbb{D}^{2}, \partial \mathbb{D}^{2}, \omega\right) .
$$

Le disque est muni de ses coordonnées polaires $(\theta, r)$ dans lesquelles la forme volume canonique s'écrit $\omega=r d r \wedge d \theta$. Regardons une application de la forme

$$
\phi_{\rho}:(\theta, r) \longmapsto(\theta+\rho(r), r)
$$


pour une certaine fonction $\rho:[0,1] \rightarrow \mathbb{R}$ lisse et nulle au voisinage de 1 (voir la figure 3.3). On vérifie facilement que $\phi_{\rho}$ est un difféomorphisme, isotope à l'identité par une isotopie fixant un voisinage du bord et qui préserve la forme volume $\omega$. C'est donc un élément de $H a m\left(\mathbb{D}^{2}, \partial \mathbb{D}^{2}, \omega\right)$. Il laisse invariant le feuilletage trivial du disque $\mathbb{D}^{2}$ en cercles concentriques, et induit la rotation d'angle $\rho(r)$ sur le cercle de rayon $r$. C'est essentiellement ce qu'on obtient de l'exemple précédent (rotation fibrée sur la sphère) après avoir éclaté un des deux points fixes.

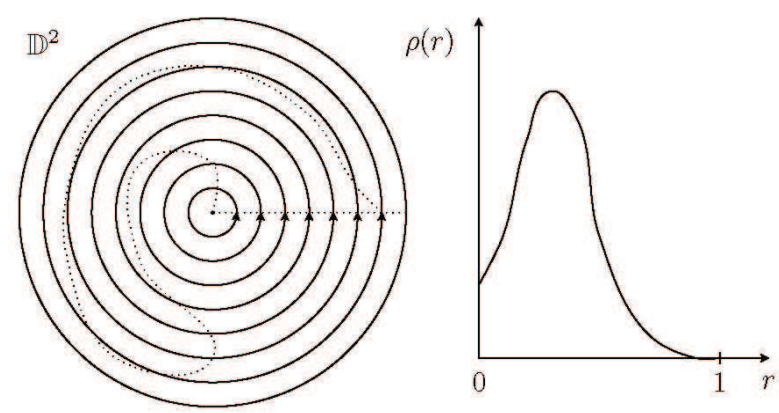

FIG. 3.3 - Exemples de difféomorphismes hamiltoniens de $\mathbb{D}^{2}$

Jusqu'à présent, on a donné des exemples simples de difféomorphismes hamiltoniens sur des surfaces où la notion coïncidait avec celle de difféomorphismes conservatifs. Voici un autre exemple (qui est en réalité toujours le même).

Exemple 3.2.6 (L'anneau $\mathbb{A})$. On peut encore reprendre l'exemple sur la sphère en éclatant les deux points fixes pour obtenir un difféomorphisme de l'anneau $\mathbb{A}=\mathbb{T} \times[0,1]$, toujours de la forme

$$
\phi_{\rho}:(\theta, r) \longmapsto(\theta+\rho(r), r)
$$

dans les coordonnées naturelles $(\theta, r) \in \mathbb{A}$, avec $\rho:[0,1] \rightarrow[0,1]$ nulle au voisinage de 0 et 1 pour respecter la condition au bord. La forme volume canonique est donnée par $\omega=d \theta \wedge d r$. Il est alors immédiat que $\phi_{\rho}$ appartient à Dif $f_{0}^{\infty}(\mathbb{A}, \partial \mathbb{A}, \omega)$ mais puisque le premier groupe d'homologie est non trivial, cela ne suffit pas à s'assurer que $\phi_{\rho}$ est un difféomorphisme hamiltonien. En revanche, un simple calcul permet de vérifier que la fonction

$$
H(r)=\int_{0}^{r} \rho(s) d s
$$


est un hamiltonien (non normalisé) qui engendre $\phi_{\rho}$. Pour pouvoir normaliser le hamiltonien, il faut demander que la fonction $\rho$ soit de moyenne nulle.

Dans les exemples précédents, on s'aperçoit que toutes les orbites d'un flot hamiltonien sont périodiques. De manière générale, ce phénomène se produit presque tout le temps (voir la figure 3.4).
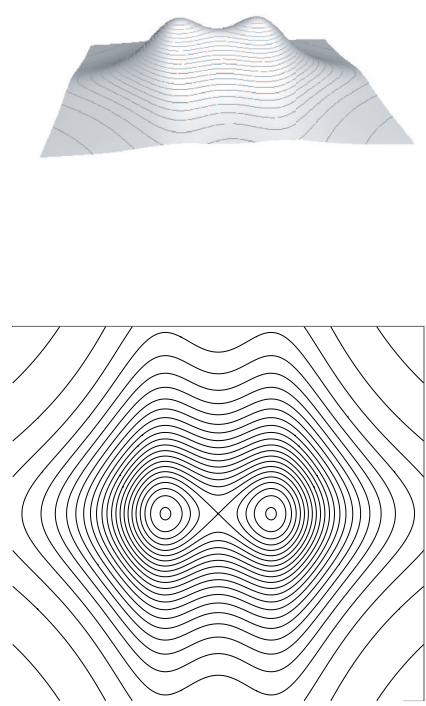

FIG. 3.4 - Graphe et orbites d'un hamiltonien autonome sur une surface

Proposition 3.2.7. Soit $H$ un hamiltonien autonome sur une surface compacte. Alors presque toute trajectoire du flot hamiltonien $\Phi_{H}$ est périodique.

Démonstration. Le flot hamiltonien préserve une mesure finie, on en déduit par le théorème de récurrence de Poincaré que l'orbite de presque tout point $x$ dans $M$ est récurrente. Prenons un tel point $x$, et supposons que la différentielle de $H$ en $x$ soit non nulle (sinon l'orbite est constante). On redresse le champ hamiltonien dans un voisinage $U$ de $x$ et on se donne 
$\Sigma$ une section transverse au flot. Par préservation de l'énergie, il est facile de vérifier que par l'application de premier retour sur $\Sigma, x$ revient sur lui-même, et donc son orbite est périodique.

Pour conclure, donnons un exemple de difféomorphisme non hamiltonien.

Exemple 3.2.8 (Le tore $\mathbb{T}^{2}$ ). Le groupe $\operatorname{Ham}\left(\mathbb{T}^{2}, \omega\right)$ est un sous-groupe propre de Dif $f_{0}^{\infty}\left(\mathbb{T}^{2}, \omega\right)$. En effet, la translation

$$
R_{\gamma}:\left(x_{1}, x_{2}\right) \longmapsto\left(x_{1}+\gamma_{1}, x_{2}+\gamma_{2}\right)
$$

avec $\gamma=\left(\gamma_{1}, \gamma_{2}\right) \in \mathbb{T}^{2}$ non nul est dans Dif $f_{0}^{\infty}\left(\mathbb{T}^{2}, \omega\right)$ mais n'est pas hamiltonienne. En effet, d'après l'exemple 3.1.7, le flux d'un tel difféomorphisme n'est pas nul.

Comme promis, démontrons maintenant que $\operatorname{Ham}(M, \omega)$ est exactement le noyau du morphisme de flux. Ce résultat est dû à Banyaga ([Ban78], mais on va suivre la preuve de [MS98]).

Théorème 3.2.9 (Banyaga). Soit $M$ une surface fermée. Un difféomorphisme $\varphi$ temps 1 d'une isotopie symplectique $\Phi=\left(\varphi^{t}\right)_{t \in[0,1]}$ est hamiltonien si et seulement si Flux $(\Phi)=0$. Dans ce cas, $\Phi$ est alors homotope à extrémités fixes à une isotopie hamiltonienne.

De manière générale, étant donnée une isotopie $\Phi=\left(\varphi^{t}\right)_{t \in[0,1]}$ préservant le volume et $\gamma$ un chemin (non nécessairement fermé) dans $M$, on peut définir le flux instantané à travers $\gamma$ au temps $t$ par

$$
\operatorname{Flux}(\Phi, t) \cdot \gamma=\int_{\gamma} i_{X_{t}} \omega
$$

avec $X_{t}$ le champ de vecteurs tangent à l'isotopie. Alors $\Phi$ est de flux nul si et seulement si son flux moyen

$$
\operatorname{Flux}(\Phi) \cdot \gamma=\int_{0}^{1}(\operatorname{Flux}(\Phi, t) \cdot \gamma) d t
$$

ne dépend que des extrémités $\gamma(0)$ et $\gamma(1)$. En revanche, $\Phi$ est une isotopie hamiltonienne si et seulement si pour tout temps $t, \varphi^{t}$ a un flux moyen nul ce qui est encore équivalent au fait que le flux instantané $\operatorname{Flux}(\Phi, t) \cdot \gamma$ ne dépend que des extrémités du chemin (il vaut alors $H_{t}(\gamma(1))-H_{t}(\gamma(0))$ ). L'idée de la preuve suivante consiste à prendre une isotopie $\Phi$ de flux moyen nul et à la modifier en gardant ses extrémités fixes de telle sorte que son flux instantané s'annule.

Démonstration du théorème 3.2.9. Notons KerFlux le noyau du morphisme de flux

$$
\text { Flux }: \widetilde{\operatorname{Diff} f_{0}^{\infty}}(M, \omega) \longrightarrow H^{1}(M, \mathbb{R}) \text {. }
$$


Il nous faut montrer que ce noyau est contenu dans $\widetilde{\operatorname{Ham}}(M, \omega)$, le revêtement universel du groupe $\operatorname{Ham}(M, \omega)$, puisque l'on dispose déjà de l'autre inclusion (qui est évidente).

Soit $\varphi$ le temps 1 d'une isotopie $\Phi$ appartenant à $\widetilde{D i f f_{0}^{\infty}}(M, \omega)$ et tel que $\operatorname{Flux}(\Phi)=0$, et $X_{t}$ le champ de vecteurs tangent à $\Phi$. L'hypothèse est donc que la 1-forme $\int_{0}^{1} i_{X_{t}} \omega d t$ est exacte, et l'on doit alors modifier l'isotopie $\Phi$ à l'intérieur de sa classe d'homotopie (à extrémités fixes) pour rendre la forme $i_{X_{t}} \omega$ exacte pour tout $t \in[0,1]$. La première étape consiste à se ramener au cas où le champ de vecteurs $\int_{0}^{1} X_{t} d t$ est identiquement nul. Soient $F$ une primitive de la 1 -forme $\int_{0}^{1} i_{X_{t}} \omega d t$ et $\varphi_{F}$ le flot hamiltonien (autonome) qu'elle engendre. Considérons alors l'isotopie $\Phi_{F}^{-1} \Phi_{H}$, et soit $X_{t}^{\prime}$ son champ de vecteurs tangents. Un calcul nous donne que $\int_{0}^{1} X_{t}^{\prime} d t=0$. Or le temps 1 de $\Phi_{F}^{-1} \Phi_{H}$ est $\varphi_{F}^{-1} \varphi$, donc puisque $\varphi_{F}^{-1}$ est hamiltonien, il suffit de prouver le théorème pour $\varphi_{F}^{-1} \varphi$. Cette discussion permet donc de se ramener au cas où $\int_{0}^{1} X_{t} d t$ est nul sur $M$. Maintenant définissons le champ de vecteurs non autonome

$$
Y_{t}=-\int_{0}^{t} X_{\lambda} d \lambda
$$

et notons, pour $t$ dans $[0,1]$ fixé, $\left(\theta_{s}^{t}\right)_{s \in[0,1]}$ le flot de $Y_{t}$, i.e.

$$
\frac{d}{d s} \theta_{s}^{t}=Y_{t} \theta_{s}^{t} \quad ; \quad \theta_{0}^{t}=1
$$

Puisque $Y_{0}=Y_{1}=0$, on a $\theta_{s}^{0}=\theta_{s}^{1}=1$ pour tout $s$ dans $[0,1]$. Définissons l'isotopie $\Psi=\left(\psi^{t}\right)_{t \in[0,1]}$ par

$$
\psi^{t}=\theta_{1}^{t} \varphi^{t}
$$

On voit facilement que $\Psi$ est homotope à extrémités fixes à $\Phi$. De plus, pour $T$ appartenant à $[0,1]$, on a

$$
\begin{aligned}
\operatorname{Flux}\left(\left(\psi^{t}\right)_{0 \leq t \leq T}\right) & =\operatorname{Flux}\left(\left(\theta_{1}^{t}\right)_{0 \leq t \leq T}\right)+\operatorname{Flux}\left(\left(\varphi^{t}\right)_{0 \leq t \leq T}\right) \\
& =\operatorname{Flux}\left(\left(\theta_{s}^{T}\right)_{0 \leq s \leq 1}\right)+\int_{0}^{T}\left[i_{X_{t}} \omega\right] d t \\
& =\left[i_{Y_{T}} \omega\right]+\int_{0}^{T}\left[i_{X_{t}} \omega\right] d t \\
& =0
\end{aligned}
$$

où l'on utilise successivement la propriété de morphisme du flux, son invariance par homotopie et le fait que $\theta_{s}^{T}$ soit le flot de $Y_{T}$. Ceci nous donne que $\int_{0}^{T} i_{X_{t}} \omega d t=0$ pour tout $T \in[0,1]$, ce qui est bien sûr équivalent au fait que $i_{X_{t}} \omega$ est exacte pour tout $t \in[0,1]$. 
On a donc obtenu une caractérisation explicite du noyau du morphisme de flux. Dans le cas à bord, on trouve de même que $\operatorname{Ham}(M, \partial M, \omega)$ est le noyau de l'application

$$
\text { Flux :Diff } f_{0}^{\infty}(M, \partial M, \omega) \longrightarrow H^{1}(M, \partial M, \mathbb{R}) .
$$

On démontrera à la fin de ce chapitre le théorème important suivant, que l'on doit à Banyaga ([Ban78], [Ban97]).

Théorème 3.2.10 (Banyaga). Le groupe $\operatorname{Ham}(M, \omega)$ est simple pour une surface fermée $M$.

Cependant, ce résultat est faux si l'on enlève l'hypothèse de fermeture sur $M$ comme on va le voir dans la section suivante.

\subsection{Second invariant de Calabi}

Regardons maintenant le cas des surfaces compactes orientables à bord. On s'intéresse à $\operatorname{Diff} f_{0}^{\infty}(M, \partial M, \omega)$, le groupe des difféomorphismes isotopes à l'identité par une isotopie préservant l'aire et fixant un voisinage du bord. Rappelons que le groupe $\operatorname{Ham}(M, \partial M, \omega)$ est un sous-groupe normal de $\operatorname{Dif} f_{0}^{\infty}(M, \partial M, \omega)$, qui de plus est propre sous la condition que le premier groupe d'homologie de la surface soit non trivial. Dans cette section, on va montrer que le groupe $\operatorname{Ham}(M, \partial M, \omega)$ n'est jamais simple.

Commençons par examiner le cas du disque $\mathbb{D}^{2}$. Puisque son premier groupe d'homologie est trivial, on a

$$
\operatorname{Diff} f_{0}^{\infty}\left(\mathbb{D}^{2}, \partial \mathbb{D}^{2}, \omega\right)=\operatorname{Ham}\left(\mathbb{D}^{2}, \partial \mathbb{D}^{2}, \omega\right) .
$$

On va alors construire un morphisme de groupes, appelé second invariant de Calabi,

$$
\text { Cal }: \operatorname{Ham}\left(\mathbb{D}^{2}, \partial \mathbb{D}^{2}, \omega\right) \longrightarrow \mathbb{R} \text {. }
$$

Prenons $\varphi$ un élément de $\operatorname{Ham}\left(\mathbb{D}^{2}, \partial \mathbb{D}^{2}, \omega\right)$, et choisissons $\lambda$ une primitive de la forme d'aire $\omega$. On vérifie facilement que la 1 -forme $\varphi^{*} \lambda-\lambda$ est fermée, donc exacte. Soit $h_{\varphi, \lambda}$ l'unique primitive de $\varphi^{*} \lambda-\lambda$ qui s'annule au voisinage du bord (par hypothèses, $\varphi$ est l'identité sur un voisinage du bord donc une primitive de $\varphi^{*} \lambda-\lambda$ est nécessairement constante sur le bord).

Définition 3.3.1. Le second invariant de Calabi de $\varphi \in \operatorname{Ham}\left(\mathbb{D}^{2}, \partial \mathbb{D}^{2}, \omega\right)$ est le réel

$$
\operatorname{Cal}(\varphi)=\frac{1}{2} \int_{\mathbb{D}^{2}} h_{\varphi, \lambda} \omega .
$$


Si $\lambda^{\prime}=\lambda+d f$ est une autre primitive, on vérifie que

$$
h_{\varphi, \lambda^{\prime}}=h_{\varphi, \lambda}+f \varphi-f
$$

et puisque $\varphi$ préserve l'aire, un simple changement de variable nous assure que $\operatorname{Cal}(\varphi)$ ne dépend effectivement que de $\varphi$. Enfin en écrivant

$$
\left(\varphi_{1} \varphi_{2}\right)^{*} \lambda-\lambda=\varphi_{2}^{*} \varphi_{1}^{*} \lambda+\varphi_{1}^{*} \lambda-\varphi_{1}^{*} \lambda-\lambda
$$

on obtient

$$
h_{\varphi_{1} \varphi_{2}, \lambda}=h_{\varphi_{2}, \varphi_{1}^{*} \lambda}+h_{\varphi_{1}, \lambda}
$$

et puisque $\varphi_{1}^{*} \lambda$ est également une primitive de la forme d'aire, on trouve

$$
\operatorname{Cal}\left(\varphi_{1} \varphi_{2}\right)=\operatorname{Cal}\left(\varphi_{1}\right)+\operatorname{Cal}\left(\varphi_{2}\right) .
$$

On vient alors de prouver la proposition suivante.

Proposition 3.3.2. Le second invariant de Calabi

$$
\text { Cal }: \operatorname{Ham}\left(\mathbb{D}^{2}, \partial \mathbb{D}^{2}, \omega\right) \longrightarrow \mathbb{R}
$$

est un morphisme de groupes.

Si on se donne maintenant une surface compacte à bord $M$ quelconque, la construction précédente s'applique encore (car la forme symplectique admet une primitive) et on en déduit un morphisme de groupe

$$
C a l: \operatorname{Ham}(M, \partial M, \omega) \longrightarrow \mathbb{R} .
$$

Cette application est évidemment continue, si $\operatorname{Ham}(M, \partial M, \omega)$ est muni de la topologie $C^{1}$. Il reste maintenant à s'assurer que ce morphisme est non trivial. Pour cela, on pourrait construire des exemples explicites de difféomorphismes hamiltoniens dont les invariants de Calabi prennent des valeurs arbitraires, mais on préfère donner une autre interprétation de ce second invariant de Calabi qui va nous être très utile par la suite.

Prenons

$$
t \in[0,1] \longmapsto \varphi^{t} \in \operatorname{Ham}(M, \partial M, \omega)
$$

une isotopie avec $\varphi^{1}=\varphi$. On sait alors que cette isotopie est uniquement définie par une fonction hamiltonienne normalisée $H:[0,1] \times M \rightarrow \mathbb{R}$.

Proposition 3.3.3. On a $\operatorname{Cal}(\varphi)=\int_{0}^{1} \int_{M} H \omega d t$.

Démonstration. Rappelons que

$$
\operatorname{Cal}(\varphi)=\frac{1}{2} \int_{M} h_{\varphi, \lambda} \omega
$$


où $h_{\varphi, \lambda}$ est l'unique primitive de $\varphi^{*} \lambda-\lambda$ qui s'annule au voisinage du bord ( $\lambda$ étant fixé). En notant $X_{t}$ le gradient symplectique associé à la fonction $H_{t}$ pour $t \in[0,1]$, on obtient facilement l'expression explicite suivante :

$$
h_{\varphi, \lambda}=\int_{0}^{1}\left(H_{t}+i_{X_{t}} \lambda\right) \varphi^{t} d t .
$$

En effet, la condition au bord est clairement vérifiée. Pour le reste, on calcule la différentielle

$$
\begin{aligned}
d\left(\int_{0}^{1}\left(H_{t}+i_{X_{t}} \lambda\right) \varphi^{t} d t\right) & =\int_{0}^{1} \varphi^{t *}\left(d H_{t}+d i_{X_{t}} \lambda\right) d t \\
& =\int_{0}^{1} \varphi^{t *}\left(i_{X_{t}} d \lambda+d i_{X_{t}} \lambda\right) d t \\
& =\int_{0}^{1} \varphi^{t *}\left(\mathcal{L}_{X_{t}} \lambda\right) d t \\
& =\int_{0}^{1} d\left(\varphi^{t *} \lambda-\lambda\right) d t \\
& =\varphi^{*} \lambda-\lambda .
\end{aligned}
$$

On a alors une nouvelle expression du second invariant de Calabi

$$
\begin{aligned}
\operatorname{Cal}(\varphi) & =\frac{1}{2} \int_{M} \int_{0}^{1}\left(H_{t}+i_{X_{t}} \lambda\right) \varphi^{t} d t \omega \\
& =\frac{1}{2} \int_{0}^{1} \int_{M}\left(H_{t}+i_{X_{t}} \lambda\right) \varphi^{t} \omega d t
\end{aligned}
$$

et puisque $\varphi^{t}$ préserve le volume pour tout $t \in[0,1]$, on trouve

$$
\operatorname{Cal}(\varphi)=\frac{1}{2} \int_{0}^{1} \int_{M}\left(H_{t}+i_{X_{t}} \lambda\right) \omega d t .
$$

Il nous reste enfin à vérifier que

$$
\left(H_{t}+i_{X_{t}} \lambda\right) \omega=2 H_{t} \omega
$$

ce que l'on obtient facilement en développant la 2-forme $i_{X_{t}}(\lambda \wedge \omega)$ qui est identiquement nulle.

Remarque 3.3.4. On donnera encore une autre interprétation de l'invariant de Calabi sur $\operatorname{Ham}\left(\mathbb{D}^{2}, \partial \mathbb{D}^{2}, \omega\right)$ dans le prochain chapitre.

Ainsi $\operatorname{Cal}(\varphi)$ n'est rien d'autre que la valeur moyenne (en temps et en espace) de la fonction hamiltonienne normalisée $H$, où $\varphi$ est le temps 1 de l'isotopie hamiltonienne engendrée par $H$. 
Le second invariant de Calabi définit donc un morphisme surjectif : pour toute valeur réelle $c$, il suffit simplement de se donner un hamiltonien autonome $H$ de valeur moyenne

$$
\int_{M} H \omega=c
$$

de telle sorte que $\varphi_{H}^{1} \in \operatorname{Ham}(M, \partial M, \omega)$ vérifie $\operatorname{Cal}\left(\varphi_{H}^{1}\right)=c$.

Remarque 3.3.5. De manière générale, le second invariant de Calabi tel qu'on l'a défini est valable pour une variété symplectique $(M, \omega)$ compacte $\grave{a}$ bord et exacte, i.e. telle que la forme symplectique $\omega$ soit exacte. Dans le cas où la variété est compacte à bord mais non exacte, l'expression

$$
\operatorname{Cal}\left(\Phi_{H}\right)=\int_{0}^{1} \int_{M} H \omega d t
$$

permet de définir un morphisme de groupe surjectif sur le revêtement universel $\widetilde{\operatorname{Ham}}(M, \partial M, \omega)$. En posant

$$
\Lambda=\operatorname{Cal}\left(\pi_{1}(\operatorname{Ham}(M, \partial M, \omega))\right) \subseteq \mathbb{R}
$$

il descend en un morphisme surjectif

$$
C a l: \operatorname{Ham}(M, \partial M, \omega) \longrightarrow \mathbb{R} / \Lambda \text {. }
$$

Le groupe $\operatorname{Ham}(M, \partial M, \omega)$ n'est jamais simple : le noyau du second invariant de Calabi, constitué des difféomorphismes hamiltoniens engendrés par des fonctions hamiltoniennes de moyenne nulle, est un sous-groupe normal propre. On en vient alors à se poser la question de la simplicité de ce sous-groupe. Encore une fois, la réponse est due à Banyaga ([Ban78], [Ban97]).

Théorème 3.3.6 (Banyaga). Soit $M$ une surface compacte à bord. Alors le noyau de l'invariant de Calabi

$$
\text { Cal }: \operatorname{Ham}(M, \partial M, \omega) \longrightarrow \mathbb{R}
$$

est un groupe simple.

Ainsi, les questions de simplicité concernant les groupes $\operatorname{Dif} f_{0}^{\infty}(M, \omega)$ et Dif $f_{0}^{\infty}(M, \partial M, \omega)$ sont toutes résolues. On conclut ce chapitre en donnant une preuve des théorèmes de Banyaga.

\subsection{Théorème de simplicité de Banyaga}

On souhaite maintenant expliquer comment on peut appliquer les idées du théorème de simplicité d'Epstein-Herman-Thurston-Mather au groupe 
des difféomorphismes hamiltoniens d'une surface $M$ dans le but de démontrer les deux théorèmes de simplicité de Banyaga (3.2.10 et 3.3.6). Il est en fait équivalent (et de notre point de vue préférable) de supposer ici que $M$ est sans bord, et de faire la distinction entre compact et non compact. Voici l'énoncé que l'on va prouver.

Théorème 3.4.1 (Banyaga). Soit $(M, \omega)$ une surface sans bord munie d'une forme symplectique. Si $M$ est compacte, alors $\operatorname{Ham}(M, \omega)$ est simple. Si $M$ n'est pas compacte, alors le noyau de

$$
C a l: \operatorname{Ham}_{c}(M, \omega) \longrightarrow \mathbb{R}
$$

est simple.

On a noté $\operatorname{Ham}_{c}(M, \omega)$ le groupe des difféomorphismes qui sont le temps 1 d'une isotopie hamiltonienne $\Phi$ à support compact. À une telle isotopie est associée une fonction hamiltonienne $H \in C^{\infty}([0,1] \times M)$ normalisée par la condition qu'il existe un compact $K \in M$ tel que $H_{t}$ soit nulle hors de $K$, pour $t$ dans $[0,1]$. Pour ne pas alourdir le texte, on notera plus simplement $\operatorname{Ham}_{c}(M, \omega)=\operatorname{Ham}(M, \omega)$, autrement dit, dans le cas non compact, on supposera implicitement que les isotopies sont à support compact.

Dans la suite, on notera également $\operatorname{Symp}_{0}(M, \omega)=\operatorname{Dif} f_{0}^{\infty}(M, \omega)$ et on parlera plutôt de difféomorphismes et d'isotopies symplectiques (la raison de ce choix vient du fait que les méthodes de cette section s'appliquent en dimension paire plus grande que deux pour des formes symplectiques et non pour des formes volumes).

Avant de nous attaquer à la preuve, on rappelle quelques résultats de géométrie symplectique dont on fera usage dans la suite. Une excellente référence est le livre [MS98].

Théorème 3.4.2 (Weinstein). Soient $(M, \omega)$ une variété symplectique et $L \hookrightarrow M$ un plongement lagrangien. Alors il existe un voisinage $N$ de $L$ dans $T^{*} L$, un voisinage $V$ de $L$ dans $M$ et un difféomorphisme symplectique

$$
\Upsilon:(N, d \lambda) \longrightarrow(V, \omega)
$$

qui commute aux plongements $L \hookrightarrow T^{*} L$ et $L \hookrightarrow M$.

Dans le théorème précèdent, le plongement $L \hookrightarrow T^{*} L$ s'obtient à travers la section nulle et $\lambda$ est la 1 -forme canonique du cotangent $T^{*} L$. On aura également besoin du résultat suivant, dont la preuve est une conséquence évidente du caractère "tautologique" de la 1 -forme canonique $\lambda$, autrement dit du fait que $\sigma^{*} \lambda=\sigma$ pour toute 1-forme $\sigma$ sur $M$, où $\sigma^{*} \lambda$ désigne le "pull-back" de $\lambda$ par l'application $\sigma: M \rightarrow T^{*} M$.

Proposition 3.4.3. Soit $\sigma$ une 1-forme sur une variété $M$. Son graphe $G r(\sigma) \subseteq T^{*} M$ est lagrangien si et seulement si $\sigma$ est fermée. 
On considère une variété symplectique $(M, \omega)$, et on munit le produit $M \times M$ de la structure symplectique $\omega \times(-\omega)$. On obtient un plongement lagrangien $M \hookrightarrow M \times M$ en identifiant $M$ à la diagonale $\Delta \subseteq M \times M$. Par le théorème de Weinstein, on trouve des voisinages $V$ de $M$ dans $M \times M$ et $N$ de $M$ dans $T^{*} M$ et un difféomorphisme symplectique $\Upsilon: V \rightarrow N$.

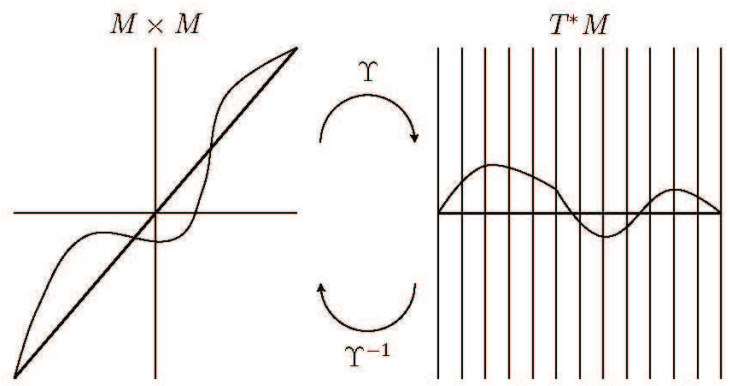

FIG. 3.5 - Théorème de Weinstein

Maintenant, si $\psi$ est un difféomorphisme symplectique de $M$ suffisamment proche (dans la topologie $C^{1}$ ) de l'identité, son graphe $\operatorname{Gr}(\psi) \subseteq$ $M \times M$ est un lagrangien suffisamment proche de la diagonale, et l'on peut supposer que $\operatorname{Gr}(\psi) \subseteq V$. Alors $\Upsilon(G r(\psi))$ est proche de l'image de la section nulle de $T^{*} M$, c'est donc le graphe d'une 1 -forme $\mathcal{W}(\psi)$, nécessairement fermée (voir la figure 3.5). Il existe ainsi un voisinage $U_{\mathcal{W}}$ de l'identité dans $\operatorname{Symp}(M, \omega)$, un voisinage $V$ de la section nulle dans l'espace vectoriel $Z^{1}(M)$ des 1-formes fermées sur $M$ et un homéomorphisme

$$
\mathcal{W}: U_{\mathcal{W}} \longrightarrow V \text {. }
$$

On dit que le couple $\left(U_{\mathcal{W}}, \mathcal{W}\right)$ est une carte de Weinstein au voisinage de l'identité. En translatant de telles cartes, on munit $\operatorname{Symp}(M, \omega)$ d'un atlas distingué. Remarquons qu'on a la propriété suivante : un difféomorphisme symplectique $\varphi \in U_{\mathcal{W}}$ est à support dans $U$ si et seulement si la 1-forme associée $\mathcal{W}(\varphi)$ s'annule hors de $U$.

Fixons $\left(U_{\mathcal{W}}, \mathcal{W}\right)$ une carte de Weinstein en l'identité. Lorsque l'on parle de difféomorphisme symplectique $C^{1}$-proche de l'identité, on sous-entend (sauf mention contraire) qu'il est au moins inclus dans $U_{\mathcal{W}}$, et de même pour les isotopies symplectiques. Un tel difféomorphisme $\varphi$ se représente donc de manière unique par une 1-forme fermée $\mathcal{W}(\varphi)$. On en déduit facilement que le groupe des difféomorphismes symplectiques d'une variété compacte est localement contractile. Donnons d'autres conséquences de l'existence de ces coordonnées. 
Proposition 3.4.4. Si $\Phi=\left(\varphi^{t}\right)_{t \in[0,1]}$ est une isotopie symplectique $C^{1}$ proche de l'identité, alors Flux $(\Phi)=-\left[\mathcal{W}\left(\varphi^{1}\right)\right]$. En particulier, $\Phi$ est une isotopie hamiltonienne si et seulement si les 1 -formes fermées $\mathcal{W}\left(\varphi^{t}\right)$ sont exactes pour tout $t$ appartenant $\grave{a}[0,1]$.

Démonstration. Pour $t$ appartenant à [0,1], définissons l'isotopie

$$
\psi^{t}=\Upsilon\left(1 \times \varphi^{t}\right) \Upsilon^{-1}
$$

où $\left(1 \times \varphi^{t}\right)_{t \in[0,1]}$ est une isotopie symplectique de plongements de $M$ dans $M \times M$ d'images contenues dans un voisinage $V$ de la diagonale, et $\Upsilon$ est un difféomorphisme symplectique qui envoie un tel voisinage sur un voisinage $N$ de la section nulle dans $T^{*} M$. Ainsi $\Psi=\left(\psi^{t}\right)_{t \in[0,1]}$ est une isotopie symplectique de plongements de la section nulle dans $N$.

Notons $\sigma_{t}=\mathcal{W}\left(\varphi^{t}\right)$ pour $t$ dans $[0,1]$. Il existe alors une famille lisse de difféomorphismes $f_{t}: M \rightarrow M$ vérifiant pour tout $t$ appartenant à $[0,1]$

$$
\psi^{t} \iota=\sigma_{t} f_{t}
$$

où $\iota: M \hookrightarrow T^{*} M$ est l'inclusion canonique à travers la section nulle. Maintenant, $\iota_{\Delta}=\Upsilon^{-1} \iota$ n'est rien d'autre que l'inclusion $M \hookrightarrow M \times M$ à travers la diagonale. On obtient ainsi

$$
\operatorname{Flux}(\Phi)=\iota_{\Delta}^{*} F \operatorname{lux}\left(\left(1 \times \varphi^{t}\right)_{t \in[0,1]}\right)=\iota_{\Delta}^{*} \Upsilon^{*} F \operatorname{lux}(\Psi)=\iota^{*} F \operatorname{lux}(\Psi) .
$$

Il faut donc calculer le flux de l'isotopie $\Psi$. Pour le moment, $\Psi$ est seulement définie sur $N$, qui est un voisinage ouvert de la section nulle dans $T^{*} M$. On peut cependant l'étendre en une isotopie symplectique dans $T^{*} M$ à support compact. Puisque $T^{*} M$ est une variété exacte, on trouve facilement que

$$
\operatorname{Flux}(\Psi)=\left[\lambda-\psi^{1 *} \lambda\right]
$$

et donc

$$
\iota^{*} \operatorname{Flux}(\Psi)=-\left[\iota^{*} \psi^{1 *} \lambda\right]=-\left[f_{1}^{*} \sigma_{1}^{*} \lambda\right]=-\left[\sigma_{1}\right]
$$

où l'on utilise le caractère tautologique de $\lambda$ et le fait que $\left[f_{1}^{*} \sigma_{1}\right]=\left[\sigma_{1}\right]$ puisque $f_{1}$ est isotope à l'identité. On trouve donc que

$$
\operatorname{Flux}(\Phi)=-\left[\sigma_{1}\right]=-\left[\mathcal{W}\left(\varphi^{1}\right)\right]
$$

et c'est ce qu'il fallait démontrer.

Ainsi un difféomorphisme hamiltonien $\varphi$ obtenu par une isotopie proche de l'identité est représenté par une forme exacte $\mathcal{W}(\varphi)$. En revanche, si on suppose seulement que $\varphi$ est proche de l'identité (sans condition sur l'isotopie) l'argument précédent ne fonctionne plus. Néanmoins, on peut encore dire quelque chose. 
Proposition 3.4.5. Soient $\varphi \in$ Symp $_{0}(M)$ un difféomorphisme $C^{1}$-proche de l'identité et $\sigma=\mathcal{W}(\varphi)$. Alors on a

$$
\varphi \in \operatorname{Ham}(M, \omega) \Longleftrightarrow[\sigma] \in \Gamma_{\omega}
$$

où $\Gamma_{\omega}=\operatorname{Flux}\left(\pi_{1}\left(\operatorname{Symp}_{0}(M, \omega)\right)\right)$ est le groupe de flux.

Démonstration. Supposons que $\varphi$ appartienne à $\operatorname{Ham}(M, \omega)$, et soit $\Phi$ une isotopie hamiltonienne définissant $\varphi$ et telle que $F l u x(\Phi)=0$. On considère l'isotopie symplectique (non nécessairement hamiltonienne) $\Psi=\left(\psi^{t}\right)_{t \in[0,1]}$ définie pour tout $t$ dans $[0,1]$ par $\mathcal{W}\left(\psi^{t}\right)=t \sigma$. Son temps 1 est donc $\varphi$ et par définition, $\Psi$ est proche de l'identité. La proposition précédente nous donne $\operatorname{Flux}(\Psi)=-[\sigma]$. Maintenant, la concaténation $\Psi \Phi^{-1}$ nous donne un lacet basé en l'identité dans $\operatorname{Symp}_{0}(M, \omega)$ et on a

$$
\operatorname{Flux}\left(\Psi \Phi^{-1}\right)=\operatorname{Flux}(\Psi)-F \operatorname{lux}(\Phi)=\operatorname{Flux}(\Psi)=-[\sigma] .
$$

Cette égalité signifie que $[\sigma]$ appartient à $\Gamma_{\omega}$.

Supposons maintenant que $[\sigma] \in \Gamma_{\omega}$. On choisit un lacet basé en l'identité $\Theta$ dans $\operatorname{Symp}_{0}(M, \omega)$ tel que $\operatorname{Flux}(\Theta)=[\sigma]$. On définit alors un chemin $\Phi=\left(\varphi^{t}\right)_{t \in[0,2]}$ en suivant le lacet $\Theta$ sur $[0,1]$ puis par $\mathcal{W}\left(\varphi^{t}\right)=[(t-1) \sigma]$ sur $[1,2]$ (cette construction est valable pour peu que $\varphi$ et donc $\sigma$ soient suffisamment petits). Le chemin $\Phi$ a clairement un flux nul, donc $\varphi=\varphi^{2}$ est un difféomorphisme hamiltonien.

Grâce à cette proposition, on peut désormais prouver un résultat particulièrement important que l'on a admis dans la section précédente pour décrire l'algèbre de Lie de $\operatorname{Ham}(M, \omega)$. Le théorème est dû à Banyaga.

Théorème 3.4.6 (Banyaga). Les isotopies hamiltoniennes sont exactement les chemins dans le groupe $\operatorname{Ham}(M, \omega)$ qui partent de l'identité.

Démonstration. Par définition, une isotopie hamiltonienne est (en particulier) un chemin dans le groupe $\operatorname{Ham}(M, \omega)$ qui part de l'identité. Pour la réciproque, on se donne un tel chemin $\Phi=\left(\varphi^{t}\right)_{t \in[0,1]}$. Par la proposition précédente, on a

$$
\sigma_{t}=\mathcal{W}\left(\varphi^{t}\right) \in \Gamma_{\omega}
$$

pour $t$ suffisamment proche de 0 , disons pour $t \in[0, \varepsilon]$. Puisque $\Gamma_{\omega}$ est dénombrable, l'application continue $t \mapsto\left[\sigma_{t}\right]$ est constante. Puisque $\sigma_{0}=0$, on trouve ainsi que $\sigma_{t}$ est exacte pour $t \in[0, \varepsilon]$, et donc

$$
\operatorname{Flux}\left(\left(\varphi^{t}\right)_{t \in[0, \varepsilon]}\right)=0 .
$$

Ceci nous assure que $\Phi$ est une isotopie hamiltonienne au voisinage de 0 , et un reparamétrage nous permet de conclure que $\Phi$ est une isotopie hamiltonienne. 
Passons maintenant à la preuve du théorème de simplicité de Banyaga. Comme dans le chapitre précédent, on commence par établir des propriétés générales de l'action du groupe $\operatorname{Ham}(M, \omega)$. La transitivité de l'action de $\operatorname{Ham}(M, \omega) \operatorname{sur} M$ est facile à obtenir.

Proposition 3.4.7. $\operatorname{Ham}(M, \omega)$ agit transitivement sur $M$.

Démonstration. Il est suffisant de pouvoir connecter deux points arbitrairement proches $x, y$ dans $M$ par un difféomorphisme hamiltonien $\varphi$. On peut donc supposer que $x, y \in U$, où $U$ est un ouvert contractile muni de coordonnées symplectiques, ce qui permet de se ramener au problème suivant : on se donne $\varepsilon>0$ et pour $a=(0,0)$ et $b=(0, \varepsilon)$ dans $\mathbb{R}^{2}$, on cherche un difféomorphisme symplectique (pour la structure symplectique canonique de $\mathbb{R}^{2}$ ), à support dans un petit disque, et qui envoie $a$ sur $b$. Un tel difféomorphisme est nécessairement hamiltonien. Notons $D_{r}$ le disque euclidien de $\mathbb{R}^{2}$ centré à l'origine et de rayon $r$. On se donne alors une fonction lisse $\eta$, à support dans $D_{2 \varepsilon}$ et qui vaut $\varepsilon$ sur $D_{\varepsilon}$. Soient $p_{2}: \mathbb{R}^{2} \rightarrow \mathbb{R}$ la projection sur la seconde composante, et $H=\eta p_{2}$. Il est alors clair que le temps 1 de $\Phi_{H}$, qui est à support dans $D_{2 \varepsilon}$, envoie $a$ sur $b$.

En revanche, la propriété de fragmentation est ici plus délicate, et c'est le point clé de la démonstration du théorème de Banyaga. De plus, il nous faudra un énoncé plus précis. En effet, si $\phi$ est un difféomorphisme hamiltonien à support dans un disque $U$ de $M$, on peut définir

$$
\operatorname{Cal}_{U}(\phi)=\int_{0}^{1} \int_{U} K \omega d t
$$

où $K$ est un hamiltonien normalisé engendrant $\phi$, l'intégrale étant bien définie car $K$ est à support compact dans $U$. En d'autres termes, le second invariant de Calabi est non trivial sur le sous-groupe $\operatorname{Ham}_{U}(M, \omega)$ des difféomorphismes hamiltoniens à support dans $U$, quand bien même il peut être identiquement nul sur $\operatorname{Ham}(M, \omega)$ (c'est le cas où $M$ est compacte). Voici donc le théorème de fragmentation symplectique (voir [Ban97], ou bien [Ban78] pour une preuve plus compliquée).

Théorème 3.4.8. Soit $(M, \omega)$ une variété symplectique. Alors pour tout recouvrement ouvert $\mathcal{U}=\left(U_{i}\right)_{i \in I}$ de $M$ par des disques et pour tout élément $\varphi$ appartenant à $\operatorname{Ham}(M, \omega)$, il existe des éléments $\varphi_{1}, \ldots, \varphi_{n}$ dans $\operatorname{Ham}(M, \omega)$ avec $\varphi_{i}$ à support dans un des éléments de $\mathcal{U}$ pour $1 \leq$ $i \leq n$ et tels que

$$
\varphi=\varphi_{1} \ldots \varphi_{n}
$$

De plus, si $M$ est compacte ou si $M$ est non compacte et $\operatorname{Cal}(\varphi)=0$, alors on peut choisir les $\varphi_{i}$ pour que $\operatorname{Cal}_{U_{i}}\left(\varphi_{i}\right)=0$ pour $1 \leq i \leq n$.

Remarque 3.4.9. Comme pour le lemme de fragmentation 2.2.1, le théorème précédent nous donne un résultat de fragmentation pour les isotopies hamiltoniennes. 
Démonstration. On se donne une famille finie de disques $U_{1}, \ldots, U_{m}$ recouvrant $M$ dans le cas compact, ou recouvrant le support compact de l'isotopie (définissant $\varphi$ ) dans le cas où $M$ est non compacte. Sans perte de généralité, on demande que $U_{i} \cap U_{i+1} \neq \emptyset$, pour $1 \leq i \leq m-1$. Enfin on associe à ce recouvrement ouvert une partition de l'unité $\lambda_{1}, \ldots, \lambda_{m}$, et pour $0 \leq i \leq m$, on considère les fonctions $\mu_{i}=\sum_{j \leq i} \lambda_{j}$.

Commençons par démontrer la propriété de fragmentation. On peut facilement se ramener au cas où $\varphi$ appartient à $U_{\mathcal{W}}$ afin de lui associer une 1-forme fermée $\mathcal{W}(\varphi)$. En définissant des 1-formes

$$
\xi_{i}=\mu_{i} \mathcal{W}(\varphi)
$$

et en remarquant que $\xi_{0}=0$ et $\xi_{m}=\mathcal{W}(\varphi)$, si l'on pouvait écrire $\xi_{i}=$ $\mathcal{W}\left(\psi_{i}\right)$ pour un difféomorphisme hamiltonien $\psi_{i}$, alors

$$
\varphi=\psi_{m}=\left(\psi_{0}^{-1} \psi_{1}\right) \ldots\left(\psi_{m-1}^{-1} \psi_{m}\right)=\varphi_{1} \ldots \varphi_{m}
$$

nous donnerait une fragmentation relativement à $U_{1}, \ldots, U_{m}$. Cependant, la 1-forme $\xi_{i}$ n'est pas nécessairement fermé, et il n'est donc pas possible de l'écrire $\xi_{i}=\mathcal{W}\left(\psi_{i}\right)$ même pour un difféomorphisme $\psi_{i}$ seulement symplectique. Pour remédier à ce problème, on remarque que l'on peut supposer en outre que $\mathcal{W}(\varphi)$ est exacte. En effet, soit $\Phi=\left(\varphi^{t}\right)_{t \in[0,1]}$ une isotopie hamiltonienne avec $\varphi^{1}=\varphi$. On choisit alors un entier naturel $N$ suffisamment grand de telle sorte que, pour tout $t$ dans $[0,1]$ et pour $0 \leq i \leq N-1$, le difféomorphisme

$$
\phi_{i}^{t}=\left(\varphi^{t\left(\frac{N-i}{N}\right)}\right)^{-1} \varphi^{t\left(\frac{N-i-1}{N}\right)}
$$

soit dans $U_{\mathcal{W}}$. On a alors

$$
\varphi=\phi_{N}^{1} \ldots \phi_{1}^{1}
$$

Pour $0 \leq i \leq N-1$, chaque $\phi_{i}^{1}$ est un difféomorphisme hamiltonien, et de plus, l'application

$$
t \in[0,1] \longmapsto\left[\mathcal{W}\left(\phi_{i}^{t}\right)\right] \in \Gamma_{\omega}
$$

est continue donc constante (car $\Gamma_{\omega}$ est dénombrable), ainsi

$$
\left[\mathcal{W}\left(\phi_{i}^{1}\right)\right]=\left[\mathcal{W}\left(\phi_{i}^{0}\right)\right]=0 .
$$

Ceci permet donc de supposer que $\varphi \in U_{\mathcal{W}}$ et que $\mathcal{W}(\varphi)$ est exacte. L'avantage est que l'on peut maintenant opérer la fragmentation sur une fonction génératrice de $\varphi$, i.e. une primitive de $\mathcal{W}(\varphi)$. En notant $B^{1}(M)$ l'espace vectoriel des 1-formes exactes sur $M$, soit $\sigma: B^{1}(M) \rightarrow C^{\infty}(M)$ une application "primitive" (i.e. une section de la différentielle extérieure) qui soit linéaire et continue : il suffit de se fixer un point $x_{0} \in M$ et de poser, pour tout $\xi \in B^{1}(M)$,

$$
\sigma(\xi)(x)=\int_{\gamma_{x, x_{0}}} \xi
$$


où $x \in M$ et $\gamma_{x, x_{0}}$ est un chemin lisse arbitraire joignant $x_{0}$ à $x$. Définissons alors une application linéaire continue

$$
\begin{aligned}
L_{i}: B^{1}(M) & \longrightarrow \\
\zeta & \longmapsto d\left(\mu_{i} \sigma(\zeta)\right) .
\end{aligned}
$$

Notons que $L_{0}=0$ et que $L_{m}$ est l'identité. Puisque $\mu_{i} \leq 1, L_{i}$ est bornée pour $0 \leq i \leq m$, il existe un voisinage $U_{0}$ inclus dans $U_{\mathcal{W}}$ tel que

$$
L_{i}\left(\mathcal{W}\left(U_{0}\right)\right) \subseteq \mathcal{W}\left(U_{\mathcal{W}}\right)
$$

Supposons alors que $\varphi$ soit dans $U_{0}$ (il suffit simplement d'augmenter l'entier $N$ du début de la preuve), et définissons

$$
\psi_{i}=\mathcal{W}^{-1}\left(L_{i}(\mathcal{W}(\varphi))\right)
$$

On vérifie immédiatement que

$$
\varphi=\psi_{m}=\left(\psi_{0}^{-1} \psi_{1}\right) \ldots\left(\psi_{m-1}^{-1} \psi_{m}\right)=\varphi_{1} \ldots \varphi_{m} .
$$

De plus, pour $1 \leq i \leq m, \psi_{i}$ est hamiltonien car $\mathcal{W}\left(\psi_{i}\right)=L_{i}(\mathcal{W}(\varphi))$ est exacte, on en déduit que $\varphi_{i}=\psi_{i-1}^{-1} \psi_{i}$ est un difféomorphisme hamiltonien à support dans $U_{i}$. Ceci prouve la propriété de fragmentation.

Passons maintenant à la seconde partie de l'énoncé. Commençons par le cas où $M$ n'est pas compacte, et supposons alors de plus que le second invariant de Calabi de $\varphi$ est nul. Par la propriété de morphisme, on obtient

$$
\operatorname{Cal}(\varphi)=\sum_{i=1}^{m} \operatorname{Cal}\left(\varphi_{i}\right)=0 .
$$

On va modifier de manière inductive la décomposition de $\varphi$ afin d'obtenir la propriété voulue. Donnons nous un disque ouvert $V \subseteq U_{1} \cap U_{2}$ (rappelons que cette intersection est non vide) tel que $\bar{V} \subseteq U_{1} \cap U_{2}$. Soit $u$ une fonction à support compact inclus dans $V$ et qui vérifie $\int_{V} u \omega=\operatorname{Cal}_{U_{1}}\left(\varphi_{1}\right)$. Considérons alors le difféomorphisme hamiltonien $\phi$, qui est le temps $1 \mathrm{du}$ flot hamiltonien associé à la fonction $u$. Par construction, on a

$$
\operatorname{Cal}_{U_{2}}(\phi)=\operatorname{Cal}_{U_{1}}(\phi)=\operatorname{Cal}_{U_{1}}\left(\varphi_{1}\right) .
$$

Remplaçons $\varphi_{1} \operatorname{par} \tilde{\varphi}_{1}=\varphi_{1} \phi^{-1}$ et $\varphi_{2}$ par $\overline{\varphi_{2}}=\phi \varphi_{2}$. On obtient

$$
\varphi=\tilde{\varphi}_{1} \overline{\varphi_{2}} \ldots \varphi_{i} \ldots \varphi_{m}
$$

avec $\tilde{\varphi}_{1}$ un difféomorphisme hamiltonien à support inclus dans $U_{1}$ et tel que $\operatorname{Cal}_{U_{1}}\left(\tilde{\varphi}_{1}\right)=0$. On construit alors $\tilde{\varphi}_{2}$ à support dans $U_{2}$ et tel que $\mathrm{Cal}_{U_{2}}\left(\tilde{\varphi}_{2}\right)=0$, et de proche en proche on arrive à une écriture

$$
\varphi=\tilde{\varphi}_{1} \tilde{\varphi}_{2} \ldots \tilde{\varphi}_{i} \ldots \overline{\varphi_{m}}
$$


avec les propriétés exigées. Observons que l'hypothèse $\operatorname{Cal}(\varphi)=0$ n'est utilisée qu'à la dernière étape pour s'assurer que $\operatorname{Cal}_{U_{m}}\left(\overline{\varphi_{m}}\right)=0$.

Il nous reste à prouver la seconde assertion du théorème dans le cas compact, que l'on va obtenir en adaptant la méthode précédente. Commençons par le cas particulier où $\varphi$ se décompose en

$$
\varphi=\varphi_{1} \varphi_{2}
$$

avec $\varphi_{i}$ à support dans $U_{i}$, pour $i=1,2$ et $M=U_{1} \cup U_{2}$. On va alors rectifier cette écriture par un difféomorphisme hamiltonien $\phi$ pour obtenir

$$
\varphi=\left(\varphi_{1} \phi^{-1}\right)\left(\phi \varphi_{2}\right)=\varphi_{1}^{\prime} \varphi_{2}^{\prime}
$$

avec $\varphi_{i}^{\prime}$ à support dans $U_{i}$ et $\operatorname{Cal}_{U_{i}}\left(\varphi_{i}^{\prime}\right)=0$ pour $i=1,2$. Notons $\alpha=$ $\operatorname{Cal}_{U_{1}}\left(\varphi_{1}\right)$ et $\beta=\operatorname{Cal}_{U_{1}}\left(\varphi_{1}\right)+\operatorname{Cal}_{U_{2}}\left(\varphi_{2}\right)$. On se donne alors une fonction plateau lisse $H$ qui vérifie

$$
H= \begin{cases}\beta\left(\int_{U_{2}} \omega\right)^{-1} & \text { sur } M \backslash U_{2} \\ 0 & \text { sur } M \backslash U_{1}\end{cases}
$$

et telle que $\int_{M} H=\alpha$. On choisit pour $\phi$ le temps $1 \mathrm{du}$ flot hamiltonien engendré par $H$ : $\phi$ est à support dans $U_{1} \cap U_{2}$, et l'on vérifie que $C_{l_{U_{1}}}(\phi)=$ $\alpha$ et $\operatorname{Cal}_{U_{2}}(\phi)=\alpha-\beta$ de telle sorte que $\operatorname{Cal}_{U_{i}}\left(\varphi_{i}^{\prime}\right)=0$ pour $i=1,2$. Maintenant dans le cas général où

$$
\varphi=\varphi_{1} \ldots \varphi_{m}
$$

avec $\varphi_{i}$ à support dans $U_{i}$ et $M=U_{1} \cup \cdots \cup U_{m}$, on écrit

$$
\varphi=\varphi_{1}\left(\varphi_{2} \ldots \varphi_{m}\right)=\varphi_{1} \psi
$$

avec $\psi$ à support dans $V=U_{2} \cup \cdots \cup U_{m}$. D'après le cas particulier précédent, on peut écrire $\varphi=\varphi_{1}^{\prime} \psi^{\prime}$ avec $\operatorname{Cal}_{U_{1}}\left(\varphi_{1}^{\prime}\right)=0$ et $\operatorname{Cal}_{V}\left(\psi^{\prime}\right)=$ 0 . Maintenant, si on se place dans la surface non compacte $V$, puisque $\mathrm{Cal}_{V}\left(\psi^{\prime}\right)=0$, on peut décomposer

$$
\psi^{\prime}=\varphi_{2}^{\prime} \ldots \varphi_{m}^{\prime}
$$

avec $\operatorname{Cal}_{U_{i}}\left(\varphi_{i}^{\prime}\right)=0$ pour $2 \leq i \leq m$ ce qui nous donne

$$
\varphi=\varphi_{1}^{\prime} \ldots \varphi_{m}^{\prime}
$$

avec $\operatorname{Cal}_{U_{i}}\left(\varphi_{i}^{\prime}\right)=0$ pour $1 \leq i \leq m$. Ceci termine la preuve du théorème.

Notons $G=\operatorname{Ham}(M, \omega, 0)$ le groupe des difféomorphismes hamiltoniens de $M$ à support compact, dont le second invariant de Calabi est nul (avec 
notre convention, cela coïncide avec le groupe $\operatorname{Ham}(M, \omega)$ pour $M$ compacte). Pour un disque $U$ inclus dans $M, G_{U}=\operatorname{Ham}_{U}(M, \omega, 0)$ est le sous-groupe des difféomorphismes à support dans $U$ qui ont un invariant de Calabi relatif à $U$ nul. Ces notations sont un peu malheureuses dans le sens où $G_{U}$ ne coïncide pas avec le sous-groupe des éléments de $G$ à support dans $U$ (mais en fait quand même partie). Elles ont néanmoins certains avantages. D'une part, le théorème de Banyaga s'énonce simplement : $G$ est simple. D'autre part, le lemme de fragmentation nous assure que si $\mathcal{U}$ est un recouvrement ouvert de $M$ par des disques, alors $\bigcup_{U \in \mathcal{U}} G_{U}$ engendre $G$. En utilisant l' "astuce" de Thurston 2.2.7, on obtient le résultat suivant.

Proposition 3.4.10. Si $G_{U}$ est parfait, alors $G$ est simple.

Démonstration. On a déjà prouvé la transitivité et la propriété de fragmentation. Il reste seulement à vérifier que si $U, V \in \mathcal{U}$ et $g \in G$ sont tels que $g(U)$ soit inclus dans $V$, alors $g G_{U} g^{-1}$ est inclus dans $G_{V}$. Soit alors $\varphi$ appartenant à $G_{U}$, temps 1 d'une isotopie hamiltonienne $\Phi_{H}$. D'après la preuve de la proposition $3.2 .2, g \varphi g^{-1}$ est le temps 1 de l'isotopie hamiltonienne associée au hamiltonien normalisé

$$
g^{*} H(t, x)=H(t, g(x))
$$

Ainsi, par un changement de variable, on obtient

$$
C a l_{V}\left(g \varphi g^{-1}\right)=\int_{0}^{1} \int_{V} H(t, g(x)) \omega d t=\int_{0}^{1} \int_{U} H(t, x) \omega d t=C a l_{U}(\varphi)=0
$$

donc $g \varphi g^{-1}$ appartient à $G_{V}$.

On peut prendre pour $U$ un disque plongé dans $M$ muni de coordonnées symplectiques, et la simplicité de $G$ dans le cas général résulte donc de la perfection de $\operatorname{Ham}\left(\mathbb{R}^{2}, \omega_{0}, 0\right)$ (ce dernier groupe étant isomorphe à $G_{U}$ ). On souhaite maintenant expliquer comment la perfection de $G$ pour une seule surface compacte $M$ implique la perfection de $\operatorname{Ham}\left(\mathbb{R}^{2}, \omega_{0}, 0\right)$.

Dorénavant, $M$ désignera une surface compacte et donc $G=\operatorname{Ham}(M, \omega)$. On définit les complexes de chaînes $B \bar{G}$ et $B \overline{G_{U}}$ de la manière suivante. Un $n$-cube est une application

$$
c: K^{n} \longrightarrow \operatorname{Diff} f_{0}^{\infty}(M, \omega)
$$

telle que $c\left(v_{0}\right)=e$ et telle que pour tout chemin $\gamma:[0,1] \rightarrow K^{n}$ avec $\gamma(0)=$ $v_{0}$, le chemin $c \gamma$ est une isotopie hamiltonienne. On note $B \bar{G}_{n}$ le $\mathbb{Z}$-module libre engendré par les $n$-cubes, quotienté par les $n$-cubes dégénérés, puis $B \bar{G}$ la réunion des $B \bar{G}_{n}$. On obtient ainsi un sous-complexe du complexe $B \overline{D i f f_{0}^{\infty}(M)}$ qui est apparu dans la section 2.2 . 
De même, $B \overline{G_{U}}$ est le sous-complexe de $B \bar{G}$ dont les $n$-cubes $c$ vérifient les conditions suivantes : pour tout $\delta$ appartenant à $K^{n}, c(\delta)$ est à support dans $U$ et pour tout chemin $\gamma:[0,1] \rightarrow K^{n}$ tel que $\gamma(0)=v_{0}$, $C a l_{U}(c \gamma)=0$. Voici le théorème de déformation symplectique, analogue au théorème 2.2.15.

Théorème 3.4.11. Soit $U$ un disque symplectique plongé dans $M$. Alors l'inclusion $G_{U} \subseteq G$ induit un isomorphisme

$$
H_{1}\left(B \overline{G_{U}}, \mathbb{Z}\right) \cong H_{1}(B \bar{G}, \mathbb{Z})
$$

La preuve de ce théorème est identique à celle du théorème 2.2 .15 (voir la remarque 2.2 .18 ). On dispose déjà de la transitivité ainsi que de la propriété de fragmentation pour les isotopies. De manière analogue au lemme 2.2.14, on a le résultat suivant (voir [Ban97] pour une preuve).

Lemme 3.4.12. Soient $V, U$ deux disques de $M$ et $f, g$ deux plongements symplectiques de $V$ dans $U$. Il existe une isotopie hamiltonienne $\left(\varphi^{t}\right)_{t \in[0,1]}$ $\grave{a}$ support dans $U$ telle que $f \varphi^{1}=g$.

Enfin, il ne nous manque plus que le résultat suivant, qui s'apparente à un lemme de fragmentation pour les familles de difféomorphismes hamiltoniens à deux paramètres (et qui est une version "symplectique" du lemme 2.2.16).

Lemme 3.4.13. Soient $\mathcal{U}=\left\{U_{1}, \ldots, U_{m}\right\}$ un recouvrement ouvert de $M$ et $c:[0,1]^{2} \rightarrow G$ un 2 -cube $C^{1}$-proche de l'identité. Alors il existe un 2 -cube $\tilde{c}$ qui vérifie les trois conditions suivantes :

(i) si $\partial$ c est à support dans $U$, alors $\partial \tilde{c}$ aussi et $[[\partial \tilde{c}]]_{U}=[[\partial c]]_{U}$;

(ii) chaque 2 -cube $\tilde{c}_{k_{1}, k_{2}}$ est à support dans $U_{k_{1}+1} \cup U_{k_{2}+1}$;

(iii) si $U_{k_{1}+1} \cap U_{k_{2}+1}=\emptyset$, alors $\partial \tilde{c}_{k_{1}, k_{2}}=0$.

Démonstration. On considère l'application

$$
\begin{aligned}
& f:[0,1]^{2} \times M \longrightarrow \\
&(\delta, x) \longmapsto(0,1]^{2} \times M \\
&\left(f_{x}(\delta), x\right)
\end{aligned}
$$

définie dans le lemme 2.2.16. Quitte à rétrécir $c$, on suppose que $c(\delta)$ appartient à $U_{0} \subseteq U_{\mathcal{W}}$ (voir la preuve du théorème 3.4 .8 ) pour tout $\delta \in[0,1]^{2}$, de telle sorte que la 1 -forme $\mathcal{W}(c(\delta))$ soit exacte. On se donne alors une fonction lisse

$$
u(\delta): M \longrightarrow \mathbb{R}
$$

telle que $\mathcal{W}(c(\delta))=d(u(\delta))$, et dont la dépendance en $\delta \in[0,1]^{2}$ est lisse. Puisque $c(0)=e$, la fonction $u(0)$ est constante, et quitte à la normaliser, on la choisit identiquement nulle. On obtient alors une fonction lisse

$$
u:[0,1]^{2} \times M \longrightarrow \mathbb{R}
$$


que l'on peut "déformer" par $f$ en posant

$$
f^{*} u=u f:[0,1]^{2} \times M \longrightarrow \mathbb{R} .
$$

A $\delta \in[0,1]^{2}$ fixé, cela nous donne une 1-forme exacte

$$
f^{*}(\mathcal{W}(c(\delta)))=d\left(f^{*} u(\delta)\right) \in \mathcal{W}\left(U_{0}\right) .
$$

On définit alors un 2-cube

$$
\begin{array}{ccc}
\tilde{c}:[0,1]^{2} & \longrightarrow & G \\
\delta & \longmapsto \mathcal{W}^{-1}\left(f^{*}(\mathcal{W}(c(\delta)))\right) .
\end{array}
$$

Puisque $f$ est l'identité sur $\{0\} \times M, f^{*} u(0)=u(0)=0$, donc $\tilde{c}(0)=e$. Pour conclure, notons que les vérifications de $(i),(i i)$ et $(i i i)$ ne dépendent que des propriétés de $f$ (ou plus exactement de $f_{x}$ ) et sont semblables à 2.2.16

$\mathrm{Au} \mathrm{vu} \mathrm{du} \mathrm{théorème} \mathrm{précédent,} \mathrm{il} \mathrm{nous} \mathrm{reste} \mathrm{une} \mathrm{dernière} \mathrm{chose} \mathrm{à} \mathrm{effectuer}$ pour démontrer le théorème de Banyaga : il suffit de trouver une variété compacte $M$ telle que le groupe $H_{1}(\operatorname{B\operatorname {Ham}(M,\omega )}, \mathbb{Z})$ soit trivial, ou, ce qui revient au même, telle que $\operatorname{Ham(M}, \omega)$ soit parfait. Bien sûr, on va choisir $M=\mathbb{T}^{2}$ et essayer de faire fonctionner les arguments du théorème de Herman-Sergeraert.

Théorème 3.4.14. Soit $\left(\mathbb{T}^{2}, \omega\right)$ le tore muni de sa forme symplectique canonique. Alors le groupe $\left.\mathrm{Ham( \mathbb {T } ^ { 2 }}, \omega\right)$ est parfait.

Démonstration. Notons Symp $p_{0}\left(\mathbb{T}^{2}, \omega\right)$ la composante connexe de l'identité du groupe des difféomorphismes symplectiques du tore $\mathbb{T}^{2}$, et $\left.\widehat{S y m p_{0}\left(\mathbb{T}^{2}\right.}, \omega\right)$ son revêtement universel. Commençons par montrer que l'inclusion plus modeste

$$
\left.\left.\left.\operatorname{Ham(\mathbb {T}^{2}}, \omega\right) \subseteq\left[\widetilde{\operatorname{Sym}} \widetilde{p_{0}\left(\mathbb{T}^{2}\right.}, \omega\right), \widehat{S y m p_{0}\left(\mathbb{T}^{2}\right.}, \omega\right)\right]
$$

résulte uniquement du théorème de Herman-Sergeraert 2.1.7. Fixons $\gamma \in \mathbb{T}^{2}$ un vecteur diophantien (que l'on choisira petit par la suite). D'après 2.1.7, il existe un voisinage $V$ de l'identité dans $\operatorname{Dif} f_{0}^{\infty}\left(\mathbb{T}^{2}\right)$ et une application

$$
s: V \longrightarrow \operatorname{Diff} f_{0}^{\infty}\left(\mathbb{T}^{2}\right) \times \mathbb{T}^{2}
$$

tels que pour $\varphi$ appartenant à $V$, en notant $s\left(R_{\gamma} \varphi\right)=(\psi, \lambda)$, on ait

$$
R_{\gamma} \varphi=R_{\lambda} \psi R_{\gamma} \psi^{-1}
$$

Pour prouver l'inclusion précédente, il suffit de supposer que l'isotopie $\Phi=$ $\left(\varphi^{t}\right)_{t \in[0,1]}$ est petite, et on la choisit alors telle que pour tout $t$ dans $[0,1]$, $R_{\gamma} \varphi^{t}$ soit dans $V$. On obtient ainsi une écriture

$$
R_{\gamma} \varphi^{t}=R_{\lambda_{t}} \psi^{t} R_{\gamma}\left(\psi^{t}\right)^{-1}
$$


avec $s\left(R_{\gamma} \varphi^{t}\right)=\left(\psi^{t}, \lambda_{t}\right)$. Puisque pour tout $t \in[0,1]$

$$
\omega=\varphi^{t *} \omega=\left(\psi^{t *}\right)^{-1} R_{\gamma}^{*} \psi^{t *} R_{\lambda_{t}-\gamma}^{*} \omega=\left(\psi^{t *}\right)^{-1} R_{\gamma}^{*} \psi^{t *} \omega
$$

on obtient

$$
\psi^{t *} \omega=R_{\gamma}^{*} \psi^{t *} \omega
$$

En notant $\omega_{t}=\psi^{t *} \omega$, on peut écrire $\omega_{t}=f_{t} \omega$ avec une fonction $f_{t}$ positive quelque soit $t \in[0,1]$. Maintenant, puisque $\gamma$ est non-résonant (i.e. $\|\mid k . \gamma\| \| \neq 0$ pour tout $k \in \mathbb{Z}^{2} \backslash\{0\}$ ), la translation $R_{\gamma}$ possède une orbite dense dans $\mathbb{T}^{2}$. La forme $\omega_{t}$ étant invariante par $R_{\gamma}$, il en est de même de la fonction $f_{t}$ qui est donc constante, nécessairement égale à 1 . On en déduit que $\omega_{t}=\omega$, pour tout $t$ dans $[0,1]$, et donc $\psi_{t}$ préserve $\omega$. L'hypothèse de flux nul sur $\varphi^{t}$ pour tout $t$ entraîne facilement que $\lambda_{t}=0$. On obtient ainsi

$$
\varphi^{t}=R_{\gamma}^{-1} \psi^{t} R_{\gamma}\left(\psi^{t}\right)^{-1}
$$

ce qui prouve que l'isotopie $\Phi$ est homotope à extrémités fixes à l'isotopie

$$
t \in[0,1] \longrightarrow R_{t \gamma}^{-1} \psi^{t} R_{t \gamma}\left(\psi^{t}\right)^{-1}
$$

et cette dernière est clairement incluse dans $\left.\left.\left[\widehat{\operatorname{Symp}\left(\mathbb{T}_{0}^{2}\right.}, \omega\right), \widehat{\operatorname{Sym} \bar{p}_{0}\left(\mathbb{T}^{2}\right.}, \omega\right)\right]$.

En utilisant le théorème de fragmentation 3.4.8, on va maintenant montrer que $\Phi$ peut s'écrire comme un produit de commutateurs dans $\left.\operatorname{Ham(\mathbb {T}^{2}}, \omega\right)$. Notons $\Psi=\left(\psi^{t}\right)_{t \in[0,1]} \in \operatorname{Symp}_{0}\left(\mathbb{T}^{2}, \omega\right), \beta=-F l u x(\Psi) \in \mathbb{T}^{2}$ et écrivons les isotopies $R_{\gamma}=\left(R_{t \gamma}\right)_{t \in[0,1]}$ et $R_{\beta}=\left(R_{t \beta}\right)_{t \in[0,1]}$. On obtient une isotopie $\Psi R_{\beta}$ et l'on peut écrire dans $\left.\widehat{S y m p_{0}\left(\mathbb{T}^{2}\right.}, \omega\right)$

$$
\Phi=\left[R_{\gamma}^{-1}, \Psi\right]=\left[R_{\gamma}^{-1}, \Psi R_{\beta}\right]
$$

puisque les translations commutent. Notons $\bar{\Psi}=\Psi R_{\beta}$, son flux étant nul, $\bar{\Psi}$ appartient à $\left.\operatorname{Ham(\mathbb {T}^{2}}, \omega\right)$. Donnons-nous $\mathcal{U}=\left\{U_{1}, \ldots, U_{m}\right\}$ un recouvrement ouvert de $M$ par des disques. Par le théorème de fragmentation symplectique, on peut écrire

$$
\bar{\Psi}=\overline{\Psi_{1}} \ldots \overline{\Psi_{m}}
$$

avec, pour $1 \leq i \leq m, \overline{\Psi_{i}}$ des isotopies hamiltoniennes à support dans $U_{i}$. Maintenant, en choisissant $\gamma$ suffisamment petit, on peut construire à l'aide d'une fonction plateau une isotopie symplectique $K_{i}$ à support dans un disque $V_{i}$ contenant $U_{i}$ (et donc de flux nul puisque $V_{i}$ est contractile) qui coïncide avec l'isotopie $R_{\gamma}^{-1} \operatorname{sur} U_{i}$. Puisque $K_{i}=R_{\gamma}^{-1} \operatorname{sur} U_{i}, K_{i}^{-1}=R_{\gamma}$ $\operatorname{sur} R_{\gamma}^{-1}\left(U_{i}\right)$ d'où

$$
R_{\gamma}^{-1} \overline{\Psi_{i}} R_{\gamma}=K_{i} \overline{\Psi_{i}} K_{i}^{-1}
$$


et il s'en suit que

$$
\begin{aligned}
\Phi & =\left[R_{\gamma}^{-1}, \bar{\Psi}\right] \\
& =\left(R_{\gamma}^{-1} \overline{\Psi_{1}} \ldots \overline{\Psi_{m}} R_{\gamma}\right)\left(\overline{\Psi_{m}}\right)^{-1} \ldots\left(\overline{\Psi_{1}}\right)^{-1} \\
& =\left(R_{\gamma}^{-1} \overline{\Psi_{1}} R_{\gamma}\right) \ldots\left(R_{\gamma}^{-1} \overline{\Psi_{m}} R_{\gamma}\right)\left(\overline{\Psi_{m}}\right)^{-1} \ldots\left(\overline{\Psi_{1}}\right)^{-1} \\
& =\left(K_{1} \overline{\Psi_{1}} K_{1}^{-1}\right) \ldots\left(K_{m} \overline{\Psi_{m}} K_{m}^{-1}\right)\left(\overline{\Psi_{m}}\right)^{-1} \ldots\left(\overline{\Psi_{1}}\right)^{-1} \\
& =\left(K_{1}\right)\left(\overline{\Psi_{1}}\right)\left(K_{1}^{-1}\right) \ldots\left(K_{m}\right)\left(\overline{\Psi_{m}}\right)\left(K_{m}^{-1}\right)\left(\overline{\Psi_{m}}\right)^{-1} \ldots\left(\overline{\Psi_{1}}\right)^{-1}
\end{aligned}
$$

De cette dernière écriture, on en déduit que $\Phi=0$ dans l'abélianisé

$$
\left.\left.\left.\left.H_{1}\left(\operatorname{Ham(\mathbb {T}^{2}}, \omega\right)\right) \cong \widehat{\operatorname{Ham}\left(\mathbb{T}^{2}\right.}, \omega\right) /\left[\widehat{\operatorname{Ham}\left(\mathbb{T}^{2}\right.}, \omega\right), \operatorname{Ham(\mathbb {T}^{2}}, \omega\right)\right]
$$

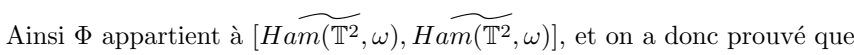

$$
\left.\left.\left.\operatorname{Ham(T^{2}}, \omega\right) \subseteq\left[\widehat{\operatorname{Ham}\left(\mathbb{T}^{2}\right.}, \omega\right), \operatorname{Ham(\mathbb {T}^{2}}, \omega\right)\right] .
$$

Pour conclure ce chapitre, évoquons la cas de la dimension supérieure. Il y a deux manières d'envisager le contexte : ou bien on considère une variété (de dimension paire) munie d'une forme symplectique auquel cas les résultats et les méthodes de cette section s'étendent immédiatement, ou bien on se place sur une variété (de dimension quelconque) munie d'une forme volume. Dans ce dernier cas, si $M$ est une variété compacte orientable de dimension $n$, on peut également définir un "morphisme de flux", à valeurs dans le $H^{n-1}(M, \mathbb{R})$, et dont le noyau constitue un sousgroupe normal. Par des techniques analogues à celles de cette section et de la section 2.2, Thurston et McDuff ont prouvé que ce noyau est simple (voir [McD82], la démonstration de Thurston étant non publiée). Notons néanmoins que les arguments dans ce cas sont beaucoup plus techniques. En effet, on peut remarquer que les preuves des théorèmes de fragmentation 2.2.1 et 3.4.8 utilisent des coordonnées "canoniques" sur les groupes Dif $f_{0}^{\infty}(M)$ et $\operatorname{Symp}_{0}(M, \omega)$, qui n'ont hélas pas d'analogues sur le groupe des difféomorphismes préservant le volume en dimension strictement plus grande que deux. 


\section{Chapitre 4}

\section{Homéomorphismes conservatifs}

Soient $M$ une surface compacte et $\mu$ une mesure de probabilité borélienne sur $M$. On dit qu'une application continue $g: M \rightarrow M$ préserve cette mesure si pour tout borélien $A$, on a $\mu(A)=\mu\left(g^{-1}(A)\right)$, i.e. si $\mu=$ $g_{*} \mu$ où $g_{*} \mu$ désigne la mesure image. On note $\operatorname{Homeo}(M, \mu)$ le groupe des homéomorphismes préservant la mesure. Dans le cas à bord, on suppose que notre mesure ne charge pas le bord $(\mu(\partial M)=0)$ et on note Homeo $(M, \partial M, \mu)$ le groupe des homéomorphismes préservant la mesure et qui sont l'identité sur un voisinage du bord.

Précisons tout de suite le contexte dans lequel on va se placer. On dit qu'une mesure $\mu$ est bonne si elle n'a pas d'atome $(\mu(\{x\})=0$ pour tout $x$ dans $M$ ) et si elle charge les ouverts non vides. Par un théorème de Von Neumann-Oxtoby-Ulam (voir [OU41]), si $\mu_{1}$ et $\mu_{2}$ sont deux telles mesures sur une variété topologique compacte, il existe alors un homéomorphisme $h$ de notre variété tel que $h_{*} \mu_{1}=\mu_{2}$ : on a donc un résultat analogue au théorème de Moser sur les formes volumes. Ainsi les propriétés algébriques et topologiques du groupe $\operatorname{Homeo}(M, \mu)$ ne dépendent pas du choix d'une bonne mesure $\mu$. En pratique, on se fixe une forme volume lisse $\omega$, et on prend la mesure $\mu(A)=\int_{A} \omega$, qui vérifie clairement les hypothèses. On note $\operatorname{Homeo}(M, \mu)$ le groupe des homéomorphismes préservant l'aire.

Dans le chapitre précédent, on a étudié et donné une réponse complète au problème de la simplicité des groupes de difféomorphismes $C^{\infty}$ de surfaces préservant le volume. On souhaite maintenant étendre ces méthodes aux groupes $\operatorname{Homeo}_{0}(M, \mu)$ et $\operatorname{Homeo}_{0}(M, \partial M, \mu)$, mais malgré plusieurs tentatives de différents auteurs, des questions importantes subsistent. 


\subsection{Approche de Fathi}

Une idée naturelle pour obtenir des résultats analogues aux groupes de difféomorphismes est d'étendre les différents invariants de Calabi aux groupes d'homéomorphismes. En effet, rappelons que pour le groupe Dif $f_{0}^{\infty}(M, \omega)$, nous avons construit un morphisme surjectif (premier invariant de Calabi)

$$
\text { Sch }: \text { Diff } f_{0}^{\infty}(M, \omega) \longrightarrow H_{1}(M, \mathbb{R}) / \Gamma_{\omega}^{\prime}
$$

qui avait permis de conclure à la non-simplicité du groupe Dif $f_{0}^{\infty}(M, \omega)$ lorsque le $H_{1}(M, \mathbb{R})$ est non trivial. Nous allons exposer dans cette section une construction de Fathi (voir [Fat80a]), qui généralise ce morphisme au groupe $\operatorname{Homeo}(M, \mu)$, et qu'il appelle morphisme de "flot de masse". Commençons par regarder quelques exemples.

Dans le cas de la sphère $\mathbb{S}^{2}$ ou du disque $\mathbb{D}^{2}$, il n'y a rien à faire puisque le premier invariant de Calabi est trivial. Pour le tore $\mathbb{T}^{2}$ et l'anneau $\mathbb{A}$, la notion de vecteur (ou de nombre) de rotation, qui est une version du premier invariant de Calabi, est bien défini pour les homéomorphismes : si $\Phi=\left(\varphi^{t}\right)_{t \in[0,1]}$ appartient à $\widetilde{H o m e o}_{0}\left(\mathbb{T}^{2}, \mu\right)$ ou à $\widetilde{H o m e o}_{0}(\mathbb{A}, \partial \mathbb{A}, \mu)$, alors la valeur moyenne de la fonction de déplacement

$\rho(\Phi, \mu)=\int_{\mathbb{T}^{2}}\left(\tilde{\varphi}^{1}(\tilde{x})-\tilde{x}\right) d \mu(x) \in \mathbb{R}^{2}$ ou $\rho(\Phi, \mu)=\int_{\mathbb{A}} p_{1}\left(\tilde{\varphi}^{1}(\tilde{x})-\tilde{x}\right) d \mu(x) \in \mathbb{R}$ donne naissance à un morphisme surjectif sur le groupe $\widehat{\text { omeo }_{0}}\left(\mathbb{T}^{2}, \mu\right)$ ou sur Homeo $_{0}\left(\mathbb{A}^{2}, \mu\right)$. Cependant cette construction ne s'adapte pas aux surfaces de genre supérieur qui ont une topologie bien plus complexe, d'où la nécessité d'une nouvelle approche.

Remarque 4.1.1. En réalité, l'invariant de Schwartzman

$$
\operatorname{Sch}(\Phi) \cdot \alpha=\int_{M}\left(\int_{\gamma_{\Phi, x}} \alpha\right) d \mu(x)
$$

peut également se définir pour un homéomorphisme d'une surface quelconque isotope à l'identité : en effet, bien que le chemin $\gamma_{\Phi, x}$ ne soit que continu, il est bien connu qu'on peut le "lisser" en gardant les extrémités fixes. Cependant cette approche n'est plus valable en dimension supérieure pour des variétés topologiques (qui n'ont pas nécessairement de structure différentiable) et il est préférable de donner une version de cet invariant qui soit purement topologique.

Avant de donner la construction générale, on va commencer par traiter l'exemple de l'anneau $\mathbb{A}$ de manière informelle. On se donne $\Theta$ la fonction angle (qui dépend d'un choix de coordonnées sur $\mathbb{A}$ )

$$
\Theta: \mathbb{A} \quad \longrightarrow \mathbb{T}
$$


et $\varphi$ un homéomorphisme de l'anneau préservant la mesure, isotope à l'identité par une isotopie préservant la mesure (et fixant le bord). Notons $\Phi=\left(\varphi^{t}\right)_{t \in[0,1]}$ cette isotopie. On peut alors relever l'homotopie

$$
\begin{aligned}
f:[0,1] \times \mathbb{A} & \longrightarrow f^{t}(x)=\Theta\left(\varphi^{t}(x)\right)-\Theta(x) \\
(t, x) & \longmapsto f^{2}
\end{aligned}
$$

en une homotopie $\bar{f}:[0,1] \times \mathbb{A} \rightarrow \mathbb{R}$, et ce de manière unique en imposant la condition $\overline{f^{0}}=0$. Si $x$ est un point de l'anneau $\mathbb{A}$,

$$
\operatorname{Var}(x, \Phi, \Theta)=\overline{f^{1}}(x) \in \mathbb{R}
$$

désigne alors sa variation angulaire sous l'isotopie. Maintenant, puisque l'on dispose d'une mesure invariante, on peut considérer la variation angulaire moyenne de tous les points de $\mathbb{A}$ en posant

$$
\operatorname{Var}(\Phi, \Theta)=\int_{\mathbb{A}} \overline{f^{1}}(x) d \mu(x)=\int_{\mathbb{A}} \operatorname{Var}(x, \Phi, \Theta) d \mu(x) \in \mathbb{R} .
$$

Cette construction est en fait valable pour toute fonction $\Theta: \mathbb{A} \rightarrow \mathbb{T}$ et il paraît raisonnable qu'elle ne dépende que de la classe d'homotopie $[\Theta]$ et de la classe d'homotopie de $\Phi$ à extrémités fixes. De plus l'application

$$
\begin{array}{rlrl}
\operatorname{Var}(\Phi): \quad[\mathbb{A}, \mathbb{T}] \cong \mathbb{Z} & \longrightarrow c & \mathbb{R} \\
\Theta & \longmapsto & \operatorname{Var}(\Phi, \Theta)
\end{array}
$$

est un morphisme de groupes, on peut donc considérer que $\operatorname{Var}(\Phi)$ est un nombre réel, et enfin l'application

$$
\begin{aligned}
& \text { Var }: \widetilde{\operatorname{Homeo}_{0}}(\mathbb{A}, \mu) \longrightarrow \mathbb{R} \\
& \Phi \quad \longmapsto \operatorname{Var}(\Phi)
\end{aligned}
$$

est également un morphisme de groupes. Le fait que ce morphisme soit surjectif (donc en particulier non trivial) se voit facilement sur un exemple : pour un réel $\alpha$ quelconque, prenons l'homéomorphisme

$$
\begin{array}{cccc}
T_{\alpha}: & \mathbb{A} & \longrightarrow \mathbb{A} \\
& (\theta, r) & \longmapsto & (\theta+\alpha \rho(r), r)
\end{array}
$$

avec une fonction $\rho$ nulle au bord et de moyenne 1. L'homéomorphisme $T_{\alpha}$ est le temps 1 de l'isotopie $T_{\alpha}=\left(T_{t \alpha}\right)_{t \in[0,1]}$ et il est clair que

$$
\operatorname{Var}\left(T_{\alpha}\right)=\alpha \int_{0}^{1} \rho(r) d r=\alpha .
$$

Il est bien sûr évident que ce que l'on vient de définir n'est rien d'autre que le nombre de rotation. C'est ce type construction qui va se généraliser à toutes les surfaces. 
Pour simplifier, on va supposer que $M$ est fermée (le cas à bord s'obtient par les modifications habituelles). Comme le suggère l'exemple précédent (et de manière analogue au premier invariant de Calabi), on commence par définir ce morphisme sur le revêtement universel $\widetilde{\operatorname{Homeo}_{0}}(M, \mu)$.

Remarque 4.1.2. On peut trouver dans [Fat80a] une preuve du fait que le groupe $\mathrm{Homeo}_{0}(M, \mu)$ est localement contractile. Ainsi, $\widetilde{\text { Homeo }_{0}}(M, \mu)$ est exactement l'ensemble des classes d'homotopies à extrémités fixes d'isotopies dans $\mathrm{Homeo}_{0}(M, \mu)$.

Notons $[M, \mathbb{T}]$ l'ensemble des classes d'homotopies d'applications de $M$ dans le cercle $\mathbb{T}$. Alors $[M, \mathbb{T}]$ a clairement une structure de groupe abélien et on a un isomorphisme

$$
\operatorname{Hom}([M, \mathbb{T}], \mathbb{R}) \cong H_{1}(M, \mathbb{R}) .
$$

En effet, il suffit de vérifier que $[M, \mathbb{T}]$ s'identifie à $H^{1}(M, \mathbb{Z})$. Si l'on choisit un générateur $\sigma$ dans $H^{1}(\mathbb{T}, \mathbb{Z})$ (une orientation sur $\mathbb{T}$ ), on obtient un morphisme de groupes

$$
\begin{array}{ccc}
{[M, \mathbb{T}]} & \longrightarrow & H^{1}(M, \mathbb{Z}) \\
g & \longmapsto & g^{*} \sigma
\end{array}
$$

qui se trouve être un isomorphisme (ce dernier point n'est pas évident, voir [Fat80a]). Prenons donc $[\Phi]$ appartenant à $\widetilde{\operatorname{Homeo}_{0}}(M, \omega)$ et $[\Theta]$ dans $[M, \mathbb{T}]$. Alors

$$
f^{t}=\Theta \varphi^{t}-\Theta: M \longrightarrow \mathbb{T}
$$

est une homotopie entre $f^{0}=0$ et $f^{1}=\Theta \varphi^{1}-\Theta$. Par la théorie des revêtements, il existe une unique homotopie

$$
\overline{f^{t}}=\overline{\Theta \varphi^{t}-\Theta}: M \longrightarrow \mathbb{R}
$$

qui relève $f^{t}$ et qui vérifie $\overline{f^{0}}=0$. On définit l'invariant de Fathi d'un élément $[\Phi]$ appartenant à $\widetilde{H_{0 m e o}}(M, \omega)$ par

$$
\mathcal{F}(\Phi) \cdot \Theta=\int_{M} \overline{f^{1}}(x) d \mu(x)=\int_{M} \overline{\Theta \varphi^{1}-\Theta}(x) d \mu(x) .
$$

Théorème 4.1.3 (Fathi). On obtient ainsi un morphisme de groupes

$$
\mathcal{F}: \widetilde{\text { fomeo }_{0}}(M, \mu) \longrightarrow H_{1}(M, \mathbb{R})
$$

continu et surjectif.

Démonstration. ([Fat80a]) Il y a plusieurs vérifications à faire. Soient $\Theta, \Theta^{\prime}$ appartenant à $C^{0}(M, \mathbb{T})$, alors on a l'égalité

$$
\left(\Theta+\Theta^{\prime}\right) \varphi^{t}-\left(\Theta+\Theta^{\prime}\right)=\left(\Theta \varphi^{t}-\Theta\right)+\left(\Theta^{\prime} \varphi^{t}-\Theta^{\prime}\right)
$$


qui passe aux relèvements

$$
\overline{\left(\Theta+\Theta^{\prime}\right) \varphi^{1}-\left(\Theta+\Theta^{\prime}\right)}=\overline{\left(\Theta \varphi^{1}-\Theta\right)}+\overline{\left(\Theta^{\prime} \varphi^{1}-\Theta^{\prime}\right)}
$$

donc $\mathcal{F}(\Phi): C^{0}(M, \mathbb{T}) \rightarrow \mathbb{R}$ est un morphisme de groupes par linéarité de l'intégrale.

Montrons maintenant que $\mathcal{F}(\Phi)$ est bien définie sur $[M, \mathbb{T}]$. Prenons une fonction $\Theta: M \rightarrow \mathbb{T}$ homotope à une constante, et soit $\bar{\Theta}$ le relevé de $\Theta$ obtenu en relevant l'homotopie. On a alors

$$
\overline{\Theta \varphi^{t}-\Theta}=\bar{\Theta} \varphi^{t}-\bar{\Theta}
$$

et donc

$$
\mathcal{F}(\Phi) . \Theta=\int_{M} \bar{\Theta} \varphi^{1}(x) d \mu(x)-\int_{M} \bar{\Theta}(x) d \mu(x)=0 .
$$

Ainsi $\mathcal{F}(\Phi)$ appartient à $\operatorname{Hom}([M, \mathbb{T}], \mathbb{R}) \cong H_{1}(M, \mathbb{R})$.

Vérifions que

$$
\mathcal{F}: \widetilde{\text { Homeo }_{0}}(M, \mu) \longrightarrow H_{1}(M, \mathbb{R})
$$

est bien définie. Si $\Psi=\left(\psi^{t}\right)_{t \in[0,1]}$ est homotope à $\Phi$, il existe $\left(H_{s}^{t}\right)_{s, t \in[0,1]}$ telle que $H_{0}^{t}=\psi^{t}$ et $H_{1}^{t}=\varphi^{t}$ pour tout $t$ dans [0,1]. On dispose maintenant d'une famille à deux paramètres $\Theta H_{s}^{t}-\Theta$ qui se relève en $\overline{\Theta H_{s}^{t}-\Theta}$ et on obtient ainsi

$$
\overline{\Theta \varphi^{1}-\Theta}=\overline{\Theta \psi^{1}-\Theta}
$$

ce qui prouve que $\mathcal{F}(\Phi)$ ne dépend que de $[\Phi]$.

Le fait que $\mathcal{F}: \widehat{\text { fomeo }_{0}}(M, \mu) \rightarrow H_{1}(M, \mathbb{R})$ soit un morphisme résulte facilement de l'égalité

$$
\Theta \varphi^{t} \phi^{t}-\Theta=\left(\Theta \varphi^{t}-\Theta\right) \phi^{t}+\left(\Theta \phi^{t}-\Theta\right)
$$

pour tout $t$ dans $[0,1]$ et du fait que $\phi^{1}$ préserve $\mu$.

Si l'on munit $\operatorname{Homeo}_{0}(M, \mu)$ de la topologie $C^{0}$ et $\operatorname{Hom}([M, \mathbb{T}], \mathbb{R})$ de la topologie faible, l'application $\mathcal{F}$ est continue, i.e.

$$
\begin{array}{ccc}
\underset{\operatorname{Homeo}_{0}}{\Phi}(M, \mu) & \longrightarrow & \mathbb{R} \\
\Phi & \longmapsto & \mathcal{F}(\Phi) . \Theta
\end{array}
$$

est continue pour toute application continue $\Theta: M \rightarrow \mathbb{T}$.

Pour conclure, il nous reste à démontrer la surjectivité. Puisque $M$ est une surface, on peut trouver une base $C_{1}, \ldots, C_{n}$ de $H_{1}(M, \mathbb{R})$ formée de cercles plongés qui admettent un voisinage tubulaire dont l'espace total $A_{i}$ est un anneau plongé. En s'appuyant sur le théorème de Von NeumannOxtoby-Ulam, on peut s'assurer que la mesure $\mu$ sur $A_{i}$ est un multiple de la mesure (de Lebesgue) standard. Alors en notant, pour $1 \leq i \leq n$ et 
$\alpha_{i} \in \mathbb{R}, T_{\alpha_{i}}$ l'homéomorphisme de l'anneau $A_{i}$ introduit auparavant, on peut définir une section de $\mathcal{F}$ par

$$
\begin{array}{ccc}
H_{1}(M, \mathbb{R}) & & { }_{\text {Homeo }_{0}}(M, \mu) \\
\alpha_{1}\left[C_{1}\right]+\cdots+\alpha_{n}\left[C_{n}\right] & \longmapsto & T_{\alpha_{1}} \cdots T_{\alpha_{n}}
\end{array}
$$

qui nous garantit la surjectivité de $\mathcal{F}$.

En notant $\Gamma_{\mu}^{\prime}=\mathcal{F}\left(\pi_{1}\left(\right.\right.$ Homeo $\left.\left._{0}(M, \mu)\right)\right) \subseteq H_{1}(M, \mathbb{R})$, on obtient un morphisme

$$
\mathcal{F}: \operatorname{Homeo}(M, \mu) \longrightarrow H_{1}(M, \mathbb{R}) / \Gamma_{\mu}^{\prime} .
$$

Vérifions que cet invariant de Fathi restreint au groupe des difféomorphismes Dif $f_{0}^{\infty}(M, \omega)$ coïncide avec le premier invariant de Calabi introduit dans la section 3.1. Comparons les deux expressions :

$$
\begin{aligned}
& \operatorname{Sch}(\Phi) \cdot \alpha=\int_{M}\left(\int_{\gamma_{\Phi, x}} \alpha\right) d \mu(x) \\
& \mathcal{F}(\Phi) \cdot \Theta=\int_{M} \overline{\Theta \varphi^{1}-\Theta}(x) d \mu(x) .
\end{aligned}
$$

En prenant $\sigma$ une orientation sur $\mathbb{T}$, il faut ainsi prouver

$$
\int_{\gamma_{\Phi, x}} \Theta^{*} \sigma=\overline{\Theta \varphi^{1}-\Theta}(x)
$$

i.e. il faut montrer que

$$
\overline{\Theta \varphi^{t}-\Theta}=\int_{0}^{t} \Theta^{*} \sigma\left(X_{s} \varphi^{s}\right) d s .
$$

D'une part, les expressions coïncident trivialement en $t=0$. D'autre part, on a pour $x$ appartenant à $M$ fixé

$$
\begin{aligned}
\Theta^{*} \sigma\left(X_{s}\left(\varphi^{s}(x)\right)\right) & =\Theta^{*} \sigma\left(\dot{\varphi}^{s}(x)\right) \\
& =\sigma\left(\frac{d}{d s}\left(\Theta \varphi^{s}\right)(x)\right) .
\end{aligned}
$$

Ainsi l'application

$$
t \in[0,1] \longmapsto \int_{0}^{t} \Theta^{*} \sigma\left(X_{s}\left(\varphi^{s}(x)\right)\right) d s \in \mathbb{R}
$$

est bien un relevé de

$$
t \in[0,1] \longmapsto\left(\Theta \varphi^{t}-\Theta\right)(x) \in \mathbb{T}
$$

qui s'annule en 0 , donc $\mathcal{F}(\Phi)=S \operatorname{ch}(\Phi)$. 
Exemple 4.1.4 (Le tore $\left.\mathbb{T}^{2}\right)$. On a l'identification $\operatorname{Hom}\left(\left[\mathbb{T}^{2}, \mathbb{T}\right], \mathbb{R}\right) \cong \mathbb{R}^{2}$. En effet, un élément de $\left[\mathbb{T}^{2}, \mathbb{T}\right]$ est représenté par une application

$$
\Theta_{a_{1}, a_{2}}: z=\left(z_{1}, z_{2}\right) \longmapsto a_{1} z_{1}+a_{2} z_{2}
$$

avec des entiers $a_{1}, a_{2}$. Donc tout élément de Hom $\left(\left[\mathbb{T}^{2}, \mathbb{T}\right], \mathbb{R}\right)$ est de la forme

$$
\Theta_{a_{1}, a_{2}} \longmapsto a_{1} x_{1}+a_{2} x_{2}
$$

pour un certain vecteur $x=\left(x_{1}, x_{2}\right)$ de $\mathbb{R}^{2}$. Dans ces conditions, on vérifie sans trop de difficultés que lorsque $\Phi$ est la translation de vecteur $\alpha, \mathcal{F}(\Phi)=$ $\alpha$, ce qui correspond bien évidemment au vecteur de rotation.

Remarque 4.1.5. Si $\Phi$ est une isotopie préservant la mesure, une application du théorème ergodique de Birkhoff analogue à celle de la section 3.1 nous donne la version asymptotique suivante :

$$
\mathcal{F}(\Phi) \cdot \Theta=\int_{M} \lim _{t \rightarrow+\infty} \frac{1}{t}\left(\overline{\Theta \varphi^{t}-\Theta}\right) d \mu
$$

où la limite existe pour $\mu$-presque tout $x$ dans $M$.

On obtient finalement que si $M$ est une surface dont le premier groupe d'homologie est non trivial, le groupe $\operatorname{Homeo}(M, \mu)$ n'est pas simple, le noyau $\operatorname{Ker}(\mathcal{F})$ étant un sous-groupe normal propre. Il est alors naturel de s'intéresser à la structure algébrique de $\operatorname{Ker}(\mathcal{F})$, ce qui inclut en particulier le cas des groupes $\mathrm{Homeo}_{0}\left(\mathbb{S}^{2}, \mu\right)$ et $\mathrm{Homeo}\left(\mathbb{D}^{2}, \partial \mathbb{D}^{2}, \mu\right)$. Il n'est pas difficile de vérifier que ce noyau est connexe et localement contractile (en admettant que $\operatorname{Homeo}(M, \mu)$ et $\operatorname{Homeo}(M, \partial M, \mu)$ possèdent ces propriétés). En utilisant une décomposition en anses, Fathi parvient à démontrer que $\operatorname{Ker}(\mathcal{F})$ a la propriété de fragmentation mais n'arrive pas à en déduire la simplicité (en dimension deux, la propriété de fragmentation peut certainement s'obtenir en adaptant les arguments du théorème de Fisher 1.2.3). En réalité, sa construction étant valable en toute dimension, il prouve que si $M$ est une variété de dimension strictement plus grande que deux, le noyau est simple $([$ Fat80a] $])$.

Théorème 4.1.6 (Fathi). Le groupe $\operatorname{Ker}(\mathcal{F})$ est simple pour $n \geq 3$.

En revanche, dans le cas qui nous intéresse, à savoir $n=2$, le problème reste ouvert. Remarquons néanmoins que grâce à l'astuce de Thurston 2.2.7, on peut se limiter au cas du disque.

Proposition 4.1.7. Le noyau de l'invariant de Fathi $\operatorname{Ker}(\mathcal{F})$ est simple pour toute surface si et seulement si le groupe $H$ omeo $\left(\mathbb{D}^{2}, \partial \mathbb{D}^{2}, \mu\right)$ est parfait.

Il suffit en effet de vérifier les hypothèses du théorème 2.2.7, et toutes ces vérifications se trouvent par exemple dans [Fat80a]. 
On va alors tenter une autre approche dans la section suivante, en essayant de généraliser le second invariant de Calabi sur le disque, qui permettrait (en cas d'existence!) de conclure à la non-simplicité de ce groupe.

\subsection{Approche de Gambaudo et Ghys}

Comme indiqué précédemment, on se concentre désormais sur le groupe

$$
\operatorname{Homeo}\left(\mathbb{D}^{2}, \partial \mathbb{D}^{2}, \mu\right)
$$

où $\mu$ est la mesure de Lebesgue sur le disque. Si $\varphi$ est un difféomorphisme du disque fixant un voisinage du bord et préservant la mesure, on sait définir son second invariant de Calabi par

$$
\operatorname{Cal}(\varphi)=\frac{1}{2} \int_{\mathbb{D}^{2}} h_{\varphi, \lambda} \omega
$$

où $h_{\varphi, \lambda}$ est une primitive de $\varphi^{*} \lambda-\lambda(\lambda$ étant une primitive de la forme d'aire) ou bien par

$$
\operatorname{Cal}(\varphi)=\int_{0}^{1} \int_{\mathbb{D}^{2}} H \omega d t
$$

avec $H$ la fonction hamiltonienne normalisée engendrant $\varphi$.

Rappelons que tout élément de $\operatorname{Homeo}\left(\mathbb{D}^{2}, \partial \mathbb{D}^{2}, \mu\right)$ est une limite $C^{0}$ de difféomorphismes préservant la mesure (voir [Sik07] par exemple). Il serait alors possible d'obtenir une extension du second invariant de Calabi au groupe Homeo $\left(\mathbb{D}^{2}, \partial \mathbb{D}^{2}, \mu\right)$ si celui-ci était continu pour la topologie $C^{0}$. Malheureusement, tel n'est pas le cas. En effet (voir [GG97]), il est facile de trouver une suite de hamiltoniens autonomes $H_{n}: \mathbb{D}^{2} \rightarrow \mathbb{R}$ à support dans le disque de rayon $1 / n$ et de moyenne constante égale à 1 . On obtient alors une suite $\varphi_{n}=\varphi_{H_{n}}^{1}$ de difféomorphismes hamiltoniens qui converge uniformément vers l'identité mais cependant $\operatorname{Cal}\left(\varphi_{n}\right)=1$ pour tout entier $n$. Il n'est donc pas possible d'étendre l'invariant de Calabi par ce simple procédé de limite.

De manière analogue à la section précédente, on va alors donner une approche, plus topologique, de cet invariant. On la doit indépendamment à Fathi ([Fat80b]) et à Gambaudo et Ghys ([GG97]).

Prenons une isotopie $\Phi=\left(\varphi^{t}\right)_{t \in[0,1]}$ dans Homeo $\left(\mathbb{D}^{2}, \partial \mathbb{D}^{2}\right)$ avec $\varphi^{1}=\varphi$ un homéomorphisme préservant la mesure. Si $x, y$ sont deux points distincts du disque $\mathbb{D}^{2}$, alors pour tout $t$ appartenant à $[0,1]$, les points $\varphi^{t}(x)$ et $\varphi^{t}(y)$ restent distincts. On obtient ainsi un vecteur non nul

$$
v_{t}=\varphi^{t}(y)-\varphi^{t}(x)
$$




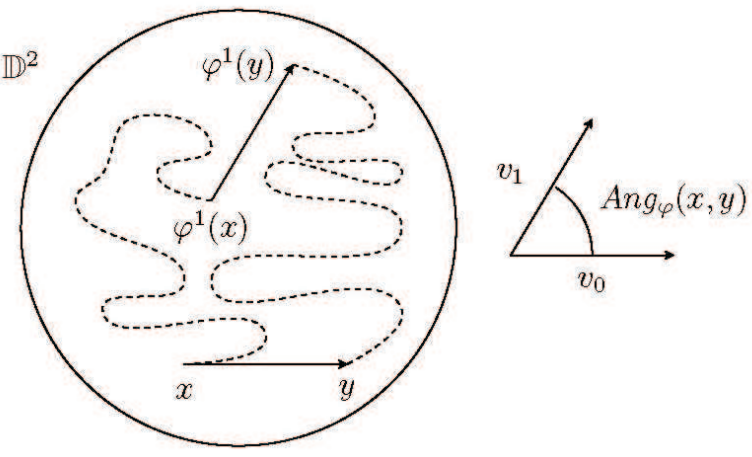

FIG. 4.1 - Variation angulaire $\operatorname{Ang}_{\varphi}(x, y)$

et on définit $\operatorname{Ang}_{\varphi}(x, y)$ comme étant la variation angulaire de $v_{t}$ lorsque $t$ varie entre 0 et 1 (voir la figure 4.1).

En notant $\Delta$ la diagonale de $\mathbb{D}^{2} \times \mathbb{D}^{2}$, on obtient une fonction

$$
\operatorname{Ang}_{\varphi}: \mathbb{D}^{2} \times \mathbb{D}^{2} \backslash \Delta \longrightarrow \mathbb{R} .
$$

Le fait que le groupe $H o m e o\left(\mathbb{D}^{2}, \partial \mathbb{D}^{2}\right)$ soit contractile nous assure que cette fonction ne dépend que de $\varphi$ et pas de l'isotopie $\Phi$. Puisque l'on dispose d'une mesure invariante, on serait tenté de définir un invariant de conjugaison en faisant la moyenne

$$
\int_{\mathbb{D}^{2} \times \mathbb{D}^{2} \backslash \Delta} \operatorname{Ang}_{\varphi}(x, y) d \mu(x) d \mu(y) .
$$

Cependant, on s'aperçoit vite que cette intégrale peut ne pas avoir de sens, car bien que la fonction $A n g_{\varphi}$ soit continue, elle n'est pas nécessairement intégrable sur la partie non compacte $\mathbb{D}^{2} \times \mathbb{D}^{2} \backslash \Delta$. Dans le cas où $\varphi$ est un difféomorphisme (de classe $C^{1}$ ), on peut s'assurer de la convergence de cette intégrale en montrant que la fonction $A n g_{\varphi}$ est bornée sur le complémentaire de la diagonale. En effet, suivant [GG97], introduisons l'ensemble $K$ des triplets $\left(x, y, \Delta_{x y}\right)$ où $(x, y) \in \mathbb{D}^{2} \times \mathbb{D}^{2}$ et $\Delta_{x y}$ est une droite du plan passant par $x$ et $y$. C'est une compactification de $\mathbb{D}^{2} \times \mathbb{D}^{2} \backslash \Delta$, dans le sens où ce dernier s'identifie à un ouvert dense de $K$. On peut alors étendre continûment notre fonction $A n g_{\varphi}$ à $K$, en définissant $A n g_{\varphi}\left(x, x, \Delta_{x}\right)$ comme étant la variation angulaire des droites $d_{x} \varphi^{t}\left(\Delta_{x}\right)$ lorsque $t$ va de 0 à 1 (il faut également s'assurer que c'est indépendant de l'isotopie). 
Nous pouvons alors énoncer le théorème suivant, dû à Fathi ([Fat80b]) et redécouvert par Gambaudo et Ghys ([GG97]).

Théorème 4.2.1 (Fathi). L'application

$$
\text { Diff } f_{0}^{\infty}\left(\mathbb{D}^{2}, \partial \mathbb{D}^{2}, \mu\right) \longrightarrow \mathbb{R}
$$

qui $\grave{a} \varphi$ associe

$$
\frac{1}{2} \int_{\mathbb{D}^{2} \times \mathbb{D}^{2} \backslash \Delta} \operatorname{Ang}_{\varphi}(x, y) d \mu(x) d \mu(y)
$$

est un morphisme de groupes qui coïncide avec le second invariant de Calabi.

Démonstration [GG97]. Il est facile de montrer que cette application est bien un morphisme de groupes. En effet, pour des difféomorphismes $\varphi$ et $\psi$, on écrit

$$
A n g_{\varphi \phi}(x, y)=A n g_{\phi}(x, y)+A n g_{\varphi}(\phi(x), \phi(y))
$$

et on utilise le fait que $\phi$ préserve l'aire pour obtenir

$$
\begin{aligned}
\int_{\mathbb{D}^{2} \times \mathbb{D}^{2} \backslash \Delta} \operatorname{Ang}_{\varphi \phi}(x, y) d \mu(x) d \mu(y)= & \int_{\mathbb{D}^{2} \times \mathbb{D}^{2} \backslash \Delta} \operatorname{Ang}_{\phi}(x, y) d \mu(x) d \mu(y) \\
& +\int_{\mathbb{D}^{2} \times \mathbb{D}^{2} \backslash \Delta} \operatorname{Ang}_{\varphi}(x, y) d \mu(x) d \mu(y) .
\end{aligned}
$$

Par le théorème de Banyaga 3.3.6 sur la simplicité du noyau du second invariant de Calabi, il existe un morphisme de groupes $\zeta: \mathbb{R} \rightarrow \mathbb{R}$ tel que pour tout $\varphi \in \operatorname{Homeo}\left(\mathbb{D}^{2}, \partial \mathbb{D}^{2}, \mu\right)$

$$
\int_{\mathbb{D}^{2} \times \mathbb{D}^{2} \backslash \Delta} \operatorname{Ang}_{\varphi}(x, y) d \mu(x) d \mu(y)=\zeta(\operatorname{Cal}(\varphi)) .
$$

De plus, $\zeta$ est continu $\operatorname{car} \operatorname{Cal}(\varphi)$ et $A n g_{\varphi}$ sont continus en $\varphi$ (pour la topologie $C^{1}$ ) donc $\zeta$ est la multiplication par une constante. Pour conclure, il suffit de montrer que ce facteur (qui n'est rien d'autre qu'une constante de normalisation) vaut $\frac{1}{2}$, ce qui peut se faire en calculant sur un hamiltonien explicite.

Remarque 4.2.2. Il existe d'autres preuves de ce résultat : voir [GG97] pour une démonstration n'utilisant pas le théorème de Banyaga, et qui consiste à montrer que les deux expressions coïncident pour des hamiltoniens de plus en plus généraux, ou bien [Fat80b] pour un argument encore différent.

Par une utilisation du théorème ergodique de Birkhoff, on obtient la version asymptotique suivante :

$$
\begin{aligned}
\int_{\mathbb{D}^{2} \times \mathbb{D}^{2} \backslash \Delta} & \operatorname{Ang}_{\varphi}(x, y) d \mu(x) d \mu(y) \\
= & \int_{\mathbb{D}^{2} \times \mathbb{D}^{2} \backslash \Delta}\left(\lim _{t \rightarrow+\infty} \frac{1}{t} A n g_{\varphi^{t}}(x, y)\right) d \mu(x) d \mu(y)
\end{aligned}
$$


après avoir étendu notre isotopie $\left(\varphi^{t}\right)_{t \in[0,1]}$ à $\mathbb{R}$. Cette relation nous permet d'obtenir une nouvelle preuve, plus géométrique, du fait que cette variation angulaire totale correspond au second invariant de Calabi (cette preuve est due à Frédéric Le Roux). Pour $\mu^{2}$-presque tout couple de points $(x, y)$, notons

$$
A(x, y)=\lim _{t \rightarrow+\infty} \frac{1}{t} A n g_{\varphi^{t}}(x, y)
$$

l'enlacement asymptotique des points $x$ et $y$. On a donc deux applications différentiables

$$
\begin{gathered}
C a l: \varphi \longmapsto \int_{0}^{1} \int_{\mathbb{D}^{2}} H \omega d t \\
\overline{C a l}: \varphi \longmapsto \frac{1}{2} \int_{\mathbb{D}^{2} \times \mathbb{D}^{2} \backslash \Delta} A(x, y) d \mu(x) d \mu(y)
\end{gathered}
$$

et l'on souhaite montrer qu'elles coïncident. Elles sont clairement égales en l'identité, il suffit alors de prouver que leur différentielle coïncident. Soit donc $\left(\varphi^{t}\right)_{t \in[0,1]}$ une courbe dans $\operatorname{Diff} f^{\infty}\left(\mathbb{D}^{2}, \partial \mathbb{D}^{2}, \mu\right)$ partant de l'identité (qui est donc une isotopie hamiltonienne), on veut montrer la relation

$$
\left.\frac{d}{d t}\right|_{t=t_{0}}\left(\operatorname{Cal}\left(\varphi^{t}\right)\right)=\left.\frac{d}{d t}\right|_{t=t_{0}}\left(\overline{\operatorname{Cal}}\left(\varphi^{t}\right)\right)
$$

pour tout $t_{0} \in[0,1]$. Puisque $\mathrm{Cal}$ et $\overline{\mathrm{Cal}}$ sont des morphismes continus pour la topologie $C^{1}$, il suffit de vérifier cette relation pour $t_{0}=0$. On obtient alors

$$
\left.\frac{d}{d t}\right|_{t=0}\left(\operatorname{Cal}\left(\varphi^{t}\right)\right)=\int_{\mathbb{D}^{2}} H_{0} \omega
$$

et

$$
\left.\frac{d}{d t}\right|_{t=0}\left(\overline{C a l}\left(\varphi^{t}\right)\right)=\int_{\mathbb{D}^{2} \times \mathbb{D}^{2} \backslash \Delta} A_{0}(x, y) d \mu(x) d \mu(y)
$$

où $A_{0}(x, y)$ est l'enlacement asymptotique des points $x$ et $y$ sous l'isotopie hamiltonienne autonome engendré par $H_{0}$. Maintenant, par la proposition 3.2 .7 , presque tout point de $M$ est sur une orbite périodique du flot hamiltonien associé à $H_{0}$. Soient $x$ un tel point, et $D_{x}$ le disque bordé par son orbite. Remarquons alors que le nombre $H_{0}(x)$ est le nombre de rotation de l'isotopie dans l'anneau $\mathbb{D}^{2} \backslash D_{x}$, et que pour un point $y$ appartenant à cet anneau, $A_{0}(x, y)$ est le nombre de rotation du point $y$ dans $\mathbb{D}^{2} \backslash D_{x}$. On trouve alors

$$
\int_{\mathbb{D}^{2} \times \mathbb{D}^{2} \backslash \Delta} A_{0}(x, y) d \mu(x) d \mu(y)=2 \int_{\mathbb{D}^{2}} H_{0} \omega .
$$

En effet, en supposant que $x$ et $y$ ont des trajectoires périodiques, on peut découper le domaine d'intégration de $A_{0}$ en trois parties : ou bien $y$ appartient à $D_{x}$, ou bien $x$ appartient à $D_{y}$, ou bien ni l'un ni l'autre et l'on 
peut facilement vérifier que ce dernier cas n'apporte aucune contribution à l'intégrale.

Même si elle ne fait pas intervenir de différentiabilité dans sa construction, la formule

$$
\operatorname{Cal}(\varphi)=\frac{1}{2} \int_{\mathbb{D}^{2} \times \mathbb{D}^{2} \backslash \Delta} \operatorname{Ang}_{\varphi}(x, y) d \mu(x) d \mu(y)
$$

n'a de sens que si $\varphi$ est un difféomorphisme. Cependant, une conséquence intéressante de cette relation est l'invariance topologique du second invariant de Calabi.

Théorème 4.2.3 (Gambaudo-Ghys). Soient $\varphi$ et $\phi$ des éléments $d u$ groupe Dif $f_{0}^{\infty}\left(\mathbb{D}^{2}, \partial \mathbb{D}^{2}, \mu\right)$ conjugués dans Homeo $\left(\mathbb{D}^{2}, \partial \mathbb{D}^{2}, \mu\right)$. Alors on a l'égalité $\operatorname{Cal}(\varphi)=\operatorname{Cal}(\phi)$.

La preuve repose sur un lemme de théorie ergodique.

Lemme 4.2.4. Soit $(X, \mu)$ un espace topologique muni d'une mesure de probabilité borélienne, $T: X \rightarrow X$ une transformation préservant la mesure, $u$ une fonction continue et $f$ une fonction intégrable tels que

$$
f=u T-u \text {. }
$$

Alors $f$ a une moyenne nulle.

Démonstration. En écrivant les sommes de Birkhoff pour la fonction $f$, on trouve

$$
\frac{1}{n} \sum_{k=0}^{n-1} f T^{k}=\frac{1}{n}\left(u T^{n}-u\right)
$$

En appliquant le théorème ergodique de Birkhoff, on trouve que le terme de gauche tend vers une fonction de moyenne égale à celle de $f$ pour $\mu$-presque tout $x$ et par le théorème de récurrence de Poincaré, on peut extraire du terme de droite une sous-suite qui converge vers 0 pour $\mu$-presque tout $x$. La fonction $f$ a donc une moyenne nulle.

Remarque 4.2.5. Le lemme suivant est en fait valable sous les hypothèses que $X$ soit un espace de probabilité et $u$ une fonction mesurable, voir [Fat80b].

Démonstration du théorème 4.2.3. On a une relation de conjugaison $\varphi=$ $\psi \phi \psi^{-1}$ pour un certain $\psi$ appartenant à Homeo $\left(\mathbb{D}^{2}, \partial \mathbb{D}^{2}, \mu\right)$ qui nous donne

$$
A n g_{\psi}(x, y)+A n g_{\varphi}(\psi(x), \psi(y))=A n g_{\phi}(x, y)+A n g_{\psi}(\phi(x), \phi(y))
$$

que l'on peut écrire sous la forme

$$
A n g_{\varphi}(\psi(x), \psi(y))-A n g_{\phi}(x, y)=A n g_{\psi}(\phi(x), \phi(y))-A n g_{\psi}(x, y) .
$$


La fonction $A n g_{\psi}$ n'est pas intégrable sur $\mathbb{D}^{2} \times \mathbb{D}^{2} \backslash \Delta$ car $\psi$ est seulement un homéomorphisme, mais elle est quand même continue. Puisque $\phi \times \phi$ préserve la mesure produit et que la fonction

$$
A n g_{\varphi}(\psi \times \psi)-A n g_{\phi}=A n g_{\psi}(\phi \times \phi)-A n g_{\psi}
$$

est intégrable (puisque $\varphi$ et $\phi$ sont des difféomorphismes), le lemme 4.2.4 nous indique qu'elle a une moyenne nulle, ce qui signifie bien que $\operatorname{Cal}(\varphi)=$ $\operatorname{Cal}(\phi)$ puisque $\psi$ préserve la mesure.

Donnons maintenant une manière plus abstraite d'envisager le problème de l'extension du second invariant de Calabi au groupe Homeo $\left(\mathbb{D}^{2}, \partial \mathbb{D}^{2}, \mu\right)$ (que l'on doit à Ghys, voir [Ghy03] et [Ghy07]).

Question 3 (Ghys). Existe-t-il une application non triviale

$$
I: C^{0}\left(\mathbb{D}^{2} \times \mathbb{D}^{2} \backslash \Delta\right) \longrightarrow \mathbb{R}
$$

linéaire et équivariante sous l'action diagonale de Homeo $\left(\mathbb{D}^{2}, \partial \mathbb{D}^{2}, \mu\right)$ sur $C^{0}\left(\mathbb{D}^{2} \times \mathbb{D}^{2} \backslash \Delta\right)$ ?

Bien sûr, si l'on se restreint aux fonctions bornées sur le complémentaire de la diagonale dans $\mathbb{D}^{2} \times \mathbb{D}^{2}$, il suffit de prendre pour $I$ l'intégration par rapport à la mesure produit $\mu^{2}$. On cherche donc à étendre cette intégration pour les fonctions qui ne sont que continues. Supposons que la réponse à la question précédente soit positive. Alors l'application

$$
\begin{array}{ccc}
\operatorname{Homeo}\left(\mathbb{D}^{2}, \partial \mathbb{D}^{2}, \mu\right) & \longrightarrow & \mathbb{R} \\
\psi & \longmapsto I\left(A n g_{\psi}\right)
\end{array}
$$

définit un morphisme de groupes non trivial (qui prolongerait le second invariant de Calabi si $I$ était une extension de l'intégration des fonctions bornées sur le complémentaire de la diagonale dans $\mathbb{D}^{2} \times \mathbb{D}^{2}$ ). Dans ce cas, le groupe Homeo $\left(\mathbb{D}^{2}, \partial \mathbb{D}^{2}, \mu\right)$ n'est pas simple, le noyau de cet "invariant de Calabi" définissant un sous-groupe normal propre de Homeo $\left(\mathbb{D}^{2}, \partial \mathbb{D}^{2}, \mu\right)$. Ce noyau contient en particulier l'ensemble

$$
H=\left\{\psi \in \operatorname{Homeo}\left(\mathbb{D}^{2}, \partial \mathbb{D}^{2}, \mu\right) \mid A n g_{\psi}=\sum_{i=1}^{n} u_{i}\left(\varphi_{i} \times \varphi_{i}\right)-u_{i}\right\}
$$

avec un entier $n$, des fonctions $u_{i} \in C^{0}\left(\mathbb{D}^{2} \times \mathbb{D}^{2} \backslash \Delta\right)$ et des homéomorphismes $\varphi_{i} \in \operatorname{Homeo}\left(\mathbb{D}^{2}, \partial \mathbb{D}^{2}, \mu\right)$, pour $1 \leq i \leq n$.

Cependant, la construction d'une telle fonctionnelle $I$ reste une question ouverte (dans [Ghy03] et [Ghy07], on pose la question "simplifiée" de l'existence d'une fonctionnelle linéaire sur l'espace des fonctions continues du disque épointé et équivariante sous l'action des homéomorphismes préservant la mesure). Mais la discussion précédente a permis de définir un sous-groupe $H$ de $H$ omeo $\left(\mathbb{D}^{2}, \partial \mathbb{D}^{2}, \mu\right)$ qui a un sens indépendemment de l'existence hypothétique de $I$ et on a la proposition suivante. 
Proposition 4.2.6 (Ghys). L'ensemble $H$ est un sous-groupe normal de Homeo $\left(\mathbb{D}^{2}, \partial \mathbb{D}^{2}, \mu\right)$ qui n'est pas réduit à l'identité.

Démonstration. Notons $G=H$ omeo $\left(\mathbb{D}^{2}, \partial \mathbb{D}^{2}, \mu\right)$ et $E=C^{0}\left(\mathbb{D}^{2} \times \mathbb{D}^{2} \backslash \Delta\right)$. Pour $u \in M$ et $\varphi \in G$, on écrit $u . \varphi=u(\varphi \times \varphi)$ (cela correspond à une action à droite de $G \operatorname{sur} E$ ). Soit $C$ l'espace des cobords, i.e. le sous-espace vectoriel de $E$ engendré par les fonctions de la forme $u . \varphi-u$, et notons [.] les classes d'équivalences dans le quotient $E / C$. Alors la relation

$$
A n g_{\phi_{1} \phi_{2}}=A n g_{\phi_{2}}+A n g_{\phi_{1}} \cdot \psi_{2}
$$

se traduit par le fait que l'application

$$
\begin{aligned}
F: G & \longrightarrow E / C \\
\varphi & \longmapsto
\end{aligned}
$$

est un morphisme de groupes. Par définition $H$ est le noyau de $F$, c'est donc un sous-groupe normal de $G$. De plus, puisque $E / C$ est abélien, $H$ contient les commutateurs de $G$ et il n'est donc pas réduit à l'identité.

Mais la question suivante, naturellement liée à la précédente, semble aussi difficile.

Question 4. $H$ est-il un sous-groupe propre de $H o m e o\left(\mathbb{D}^{2}, \partial \mathbb{D}^{2}, \mu\right)$ ?

On va alors changer de point de vue, et au lieu d'essayer d'étendre les invariants de Calabi, on va tenter de définir un groupe d'homéomorphismes "hamiltoniens". Avant cela, il nous faudra examiner un peu plus en détail le groupe des difféomorphismes hamiltoniens.

\subsection{Distance de Hofer}

Dans cette section, on poursuit l'étude du groupe des difféomorphismes hamiltoniens. Plus précisément, on y introduit une distance bi-invariante, appelée distance de Hofer, qui trouve son origine dans la recherche d'orbites périodiques pour les systèmes hamiltoniens (ou plus précisément de caractéristiques fermées dans les hypersurfaces des variétés symplectiques).

Cependant, le but ici est seulement de donner quelques définitions et propriétés élémentaires de la distance de Hofer dont on aura besoin dans la section suivante. Pour cette partie, on pourra consulter les références [MS98], [HZ94], [Pol01] et [AK98]. On se donne $(M, \omega)$ une variété symplectique de dimension quelconque.

Soit $\varphi$ un difféomorphisme hamiltonien. Si l'on considère $\varphi$ comme un "mouvement mécanique", une question naturelle est alors de savoir quelle est l' "énergie minimale" nécessaire pour engendrer $\varphi$. 
Par définition, il existe un hamiltonien $H$ appartenant à $\mathcal{H}$ qui engendre une isotopie $\Phi_{H}=\left(\varphi_{H}^{t}\right)_{t \in[0,1]}$ avec $\varphi_{H}^{1}=\varphi$. Choisissons une norme $\|$. $\|$ sur l'espace vectoriel $\mathcal{A}$. Cela permet de mesurer l' "énergie" d'un hamiltonien $H$ par l'intégrale $\int_{0}^{1}\left\|H_{t}\right\| d t$. On définit alors l' "énergie" de $\varphi$ par

$$
E(\varphi)=\inf \left\{\int_{0}^{1}\left\|H_{t}\right\| d t \mid \varphi_{H}^{1}=\varphi, H \in \mathcal{H}\right\}
$$

et ceci permet de donner une réponse à la question posée. On peut considérer l'énergie de $\varphi$ comme une distance à l'identité, et en décrétant l'invariance à gauche, on obtient une application

$$
d: \operatorname{Ham}(M, \omega) \times \operatorname{Ham}(M, \omega) \longrightarrow[0,+\infty[
$$

définie par

$$
d(\psi, \varphi)=d\left(1, \psi^{-1} \varphi\right)=E\left(\psi^{-1} \varphi\right) .
$$

Voici une autre manière de comprendre cette distance $d(\psi, \varphi)$. On rappelle qu'une structure de Finsler sur une variété $N$ est la donnée, pour tout point $x$ appartenant à $N$, d'une norme $\|.\|_{x}$ sur $T_{x} N$ qui dépend de manière lisse du point $x$ (c'est donc une notion un peu plus générale que celle de métrique riemannienne). Maintenant, si l'on considère $\operatorname{Ham}(M, \omega)$ comme un groupe de Lie, $\mathcal{A}$ s'identifie à son espace tangent à l'identité (voir la section 3.2) et la norme $\|$.$\| s'étend par invariance à gauche en une structure de Finsler$ sur $\operatorname{Ham}(M, \omega)$. Comme dans le cas riemannien, on peut ainsi définir la longueur des courbes dans $\operatorname{Ham}(M, \omega)$ (en particulier, $\int_{0}^{1}\left\|H_{t}\right\| d t$ n'est rien d'autre que la longueur de la courbe $\left.t \in[0,1] \mapsto \varphi_{H}^{t} \in \operatorname{Ham}(M, \omega)\right)$ puis définir la distance $d(\psi, \varphi)$ comme la longueur minimale d'une courbe reliant $\psi$ et $\varphi$.

Pour les groupes de Lie de dimension finie, il est toujours plus utile d'avoir une distance bi-invariante. On sait que cela revient à demander l'invariance de $\|$.$\| sous l'action adjointe, dans notre cas, on veut donc$ $\|H\|=\|H \phi\|$ pour tout difféomorphisme hamiltonien $\phi$. Remarquons qu'une telle propriété impose ici une sévère restriction sur le choix de $\|$.$\| ,$ à savoir la "nature $C^{0}$ " de $\|H\|$, qui ne doit pas faire intervenir de dérivées de $H$.

Proposition 4.3.1. L'application d: $\operatorname{Ham}(M, \omega) \times \operatorname{Ham}(M, \omega) \rightarrow[0,+\infty[$ ainsi définie vérifie les propriétés suivantes, pour tout $\varphi, \psi, \phi \in$ $\operatorname{Ham}(M, \omega)$ :

(i) $\varphi=\psi \Rightarrow d(\varphi, \psi)=0$;

(ii) $d(\varphi, \psi)=d(\psi, \varphi)$;

(iii) $d(\varphi, \theta) \leq d(\varphi, \psi)+d(\psi, \theta)$;

(iv) $d(\varphi, \psi)=d\left(\theta \varphi \theta^{-1}, \theta \psi \theta^{-1}\right)$. 
Démonstration. Le point $(i)$ est trivial. Pour $(i i)$, (iii) et $(i v)$, il suffit de montrer respectivement que $E(\varphi)=E\left(\varphi^{-1}\right), E(\varphi \psi) \leq E(\varphi)+E(\psi)$ et $E\left(\phi \varphi \phi^{-1}\right)=E(\varphi)$ ce qui résulte facilement des formules définissant $\bar{H}$, $H \# K$, et $\phi^{*} H$ (voir la proposition 3.2.2) ainsi que la propriété d'invariance $\|H\|=\|H \phi\|$ pour tout $\phi \in \operatorname{Ham}(M, \omega)$.

En particulier, $d$ définit seulement une pseudo-distance $\operatorname{sur} \operatorname{Ham}(M, \omega)$, on ne peut pas dire de manière générale si $d$ est non dégénérée, i.e. si

$$
d(\varphi, \psi)=0 \Longrightarrow \varphi=\psi
$$

Cela va dépendre du choix de la norme $\|.\| \operatorname{sur} \mathcal{A}$. Voici deux exemples que l'on étudiera plus en détail par la suite :

(1) la norme $L^{p}, 1 \leq p<\infty$ définie par

$$
\|H\|_{p}=\left(\int_{M}|H|^{p} \omega\right)^{1 / p}
$$

(2) la norme d'oscillation

$$
O s c(H)=\sup _{x \in M} H(x)-\inf _{x \in M} H(x) .
$$

On aurait pu également choisir la norme $C^{0}$

$$
\|H\|_{C^{0}}=\sup _{x \in M}|H(x)|
$$

mais par normalisation des hamiltoniens, on obtient facilement l'inégalité

$$
\|H\|_{C^{0}} \leq O s c(H) \leq 2\|H\|_{C^{0}}
$$

et donc l'équivalence entre la norme $C^{0}$ et l'oscillation. Le choix de la norme d'oscillation est motivé par la recherche d'orbites périodiques des systèmes hamiltoniens (voir [HZ94]).

On note $d_{p}$ et $d_{\infty}$ les pseudo-distances définies par ces normes. La pseudodistance $d_{\infty}$ est appelée distance de Hofer. On verra par la suite qu'elle est effectivement non dégénérée, ce qui n'est pas le cas de $d_{p}$.

Associée à la norme d'oscillation sur $\mathcal{A}$, on définit la norme de Hofer d'un hamiltonien $H$ appartenant à $\mathcal{H}$ par

$$
\|H\|_{1, \infty}=\int_{0}^{1} O s c\left(H_{t}\right) d t
$$

de telle sorte que

$$
d_{\infty}(1, \varphi)=\inf \left\{\|H\|_{1, \infty} \mid \varphi_{H}^{1}=\varphi, H \in \mathcal{H}\right\} .
$$


On aurait pu encore choisir d'autres normes sur $\mathcal{H}$, comme par exemple

$$
\sup _{t \in[0,1]}\left(O s c\left(H_{t}\right)\right)
$$

ou bien la norme équivalente

$$
\sup _{(t, x) \in[0,1] \times M}|H(t, x)| .
$$

Ces deux dernières normes ne sont pas équivalentes à $\|\cdot\|_{1, \infty}$, cependant, les distances induites sur $\operatorname{Ham}(M, \omega)$ coïncident (voir [Pol98]).

Proposition 4.3.2 (Polterovich). Pour $\varphi$ appartenant à $\operatorname{Ham}(M, \omega)$, on $a$

$$
d_{\infty}(1, \varphi)=\inf \left\{\sup _{t \in[0,1]}\left(O s c\left(H_{t}\right)\right) \mid \varphi_{H}^{1}=\varphi, H \in \mathcal{H}\right\} .
$$

La définition originale de Hofer (voir [Hof90]) était

$$
\|H\|=\left(\sup _{(x, t) \in M \times[0,1]} H_{t}(x)\right)-\left(\inf _{(x, t) \in M \times[0,1]} H(x)\right)
$$

et cette dernière est clairement équivalente à $\|.\|_{C^{0}}$. Dans la suite, on écrit simplement $\|\cdot\|=\|\cdot\|_{1, \infty}$ pour la norme de Hofer.

Remarque 4.3.3. Si d est une pseudo-distance bi-invariante sur le groupe $\operatorname{Ham}(M, \omega)$, on note

$$
G_{d}=\{\varphi \in \operatorname{Ham}(M, \omega) \mid d(1, \varphi)=0\} .
$$

Par les propriétés d'invariance de d, il est immédiat que $G_{d}$ est un sousgroupe normal de $\operatorname{Ham}(M, \omega)$. Si $M$ est fermée, on sait que $\operatorname{Ham}(M, \omega)$ est simple, donc $G_{d}$ est trivial ou $G_{d}=\operatorname{Ham}(M, \omega)$. En particulier pour prouver la non dégénérescence de d sur une variété fermée donnée, il suffit d'exhiber un seul difféomorphisme $\varphi$ vérifiant $d(1, \varphi)>0$.

De manière générale, la non-dégénérescence d'une pseudo-distance biinvariante $d$ sur le groupe des difféomorphismes hamiltoniens est liée à la notion d'énergie de déplacement.

Définition 4.3.4. On appelle énergie de déplacement d'une partie $U$ incluse dans $M$ la quantité définie par

$$
e_{d}(U)=\inf \{d(1, \varphi) \mid \varphi \in \operatorname{Ham}(M, \omega) \text { et } \varphi(U) \cap U=\emptyset\}
$$

si cet ensemble n'est pas vide, et $e_{d}(U)=+\infty$ dans le cas contraire. 


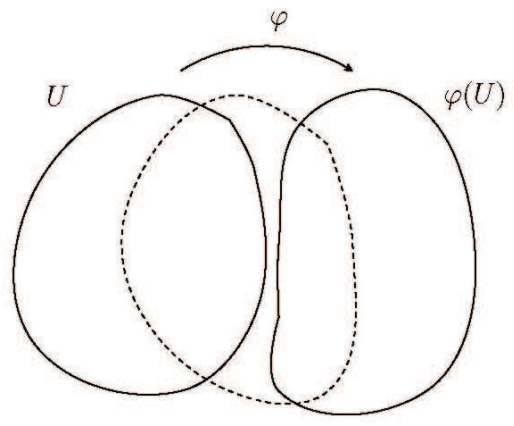

FIG. 4.2 - Énergie de déplacement

Cette quantité représente l'énergie minimale dont un difféomorphisme hamiltonien a besoin pour déformer $U$ en un ensemble qui lui soit disjoint, d'où le terme énergie de déplacement (voir la figure 4.2). L'intérêt de cette notion réside dans le théorème suivant ([Pol01], [EP93]).

Théorème 4.3.5 (Eliashberg-Polterovich). La pseudo-distance d est non dégénérée si et seulement si $e_{d}(U)>0$ pour tout ouvert $U$ non vide.

Démonstration. ([Pol01]) Supposons que $e_{d}$ charge les ouverts non vides. Si $\varphi$ appartenant à $\operatorname{Ham}(M, \omega)$ est différent de l'identité, il existe un point $x$ dans $M$ tel que $\varphi(x) \neq x$ et alors par continuité $\varphi$ déplace une petite boule $U$ centrée en $x$, d'où $d(1, \varphi) \geq e_{d}(U)>0$ et donc $d$ est non dégénérée. Pour la réciproque, on procède en deux étapes.

(a) Si $U$ est un ouvert non vide, il existe $\varphi, \psi \in \operatorname{Ham}(M, \omega)$ à support dans $U$ tels que $[\varphi, \psi] \neq 1$.

Commençons par remarquer que si deux hamiltoniens autonomes $H$ et $K$ engendrent des flots qui commutent, alors $\{H, K\}=0$. Soit $x$ un point de $U$, choisissons deux vecteurs $\xi, \eta \in T_{x} U$ tels que $\omega(\xi, \eta) \neq 0$. En prenant des coordonnées symplectiques au voisinage de $x$, on trouve deux germes de fonctions $H$ et $K$ vérifiant $X_{H}(x)=\xi$ et $X_{K}(x)=\eta$, puis on étend ces fonctions sur $M$ par une fonction plateau. Quitte à ajouter une constante pour normaliser, on trouve des hamiltoniens qui vérifient $\{H, K\} \neq 0$ car

$$
\{H, K\}(x)=\omega\left(X_{H}(x), X_{K}(x)\right)=\omega(\xi, \eta) \neq 0
$$

donc leurs flots, qui sont à support dans $U$, ne commutent pas. Ceci prouve l'assertion. 
(b) Si $U$ est un ouvert non vide et $\varphi, \psi \in \operatorname{Ham}(M, \omega)$ sont à support dans $U$, alors on a $e_{d}(U) \geq 1 / 4 d(1,[\varphi, \psi])$.

Le théorème est alors une conséquence immédiate de ces deux assertions. Il nous reste donc à démontrer cette seconde affirmation. On peut supposer $e_{d}(U)<\infty$ (sinon l'inégalité serait triviale), il existe alors un difféomorphisme hamiltonien $h$ qui déplace $U$. Notons $\theta=\left[\varphi, h^{-1}\right]$. On peut alors vérifier que $[\varphi, \psi]=[\theta, \psi]$. Ainsi

$$
\begin{aligned}
d(1,[\varphi, \psi]) & =d(1,[\theta, \psi]) \\
& =d\left(\theta^{-1}, \psi \theta \psi^{-1}\right) \\
& \leq d\left(1, \theta^{-1}\right)+d\left(1, \psi \theta \psi^{-1}\right) \\
& =2 d(1, \theta) \\
& \leq 4 d(1, h) .
\end{aligned}
$$

En minimisant sur les $h$ qui déplacent $U$, on obtient l'inégalité voulue.

Ce théorème va nous être fort utile pour étudier la dégénérescence ou non des pseudo-distances $d_{\infty}$ et $d_{p}$.

Théorème 4.3.6. On a $e_{d_{\infty}}(U)>0$ pour tout ouvert $U$ non vide, i.e. la distance de Hofer est non dégénérée.

Ce théorème est très difficile, et il semble que les preuves existantes restent encore assez mystérieuses (voir la discussion dans [Pol01]). On le doit à Hofer pour $M=\mathbb{R}^{2 n}$ ([Hof93], [HZ94]) et à Lalonde-McDuff dans le cas général ([LM95]). Dans tous les cas, la preuve repose sur la notion de capacité symplectique (voir [Vit89], [HZ94]).

Définition 4.3.7. Une capacité symplectique est une application qui a toute variété symplectique $(M, \omega)$ associe un nombre $c(M, \omega) \in[0, \infty]$ et qui vérifie les axiomes suivants:

(A1) si $(M, \omega)$ se plonge symplectiquement dans $\left(M^{\prime}, \omega^{\prime}\right)$, alors

$$
c(M, \omega) \leq c\left(M^{\prime}, \omega^{\prime}\right) \quad \text { (monotonie) }
$$

(A2) on a $c(M, \alpha \omega)=|\alpha| c(M, \omega)$ pour tout réel $\alpha \neq 0$ (homogénéité);

(A3) si on note $B^{2 n}(r)$ la boule euclidienne de rayon $r$ dans $\mathbb{R}^{2 n}$ et $Z^{2 n}(r)=B^{2}(r) \times \mathbb{R}^{2 n-2}$ le cylindre symplectique, alors

$$
c\left(B^{2 n}(1), \omega_{0}\right)>0 \quad ; \quad c\left(Z^{2 n}(1), \omega_{0}\right)<\infty \quad \text { (non-trivialité). }
$$

En cas d'existence, le premier axiome nous assure qu'un tel nombre est en particulier un invariant symplectique et le dernier axiome nous interdit d'avoir le volume comme capacité symplectique (en dimension strictement plus grande que deux bien sûr), ce qui permet de faire une distinction claire entre la géométrie symplectique et la géométrie sous-jacente associé 
à la préservation du volume. Les capacités symplectiques existent, et elles sont même nombreuses. Le premier exemple est dû à Gromov ([Gro85]), où

$$
c_{G}(M, \omega)=\sup \left\{\pi r^{2} \mid\left(B^{2 n}(r), \omega_{0}\right) \hookrightarrow(M, \omega)\right\} .
$$

Cette application vérifie clairement les deux premiers axiomes, quand à la non-trivialité, c'est une conséquence d'un théorème fondamental (et très difficile) de Gromov, qui inaugura l'utilisation des courbes pseudoholomorphes en géométrie symplectique.

Théorème 4.3.8 (Gromov). Il existe un plongement symplectique

$$
\left(B^{2 n}(r), \omega_{0}\right) \hookrightarrow\left(Z^{2 n}(R), \omega_{0}\right)
$$

seulement si $r \leq R$.

On en déduit ainsi que

$$
c_{G}\left(B^{2 n}(1), \omega_{0}\right)=c_{G}\left(Z^{2 n}(1), \omega_{0}\right)=\pi .
$$

La non-dégénérescence de la distance de Hofer dans le cas général est alors une conséquence de l'inégalité d' "énergie-capacité" suivante, que l'on doit à Lalonde et McDuff.

Théorème 4.3.9 (Lalonde-McDuff). Soient $(M, \omega)$ une variété symplectique et $U$ une partie de $M$, alors

$$
e_{d_{\infty}}(U) \geq \frac{1}{2} c_{G}(U)
$$

Pour $M=\mathbb{R}^{2 n}$, il existe d'autres capacités symplectiques, de nature variationnelles, construites dans un premier temps par Ekeland et Hofer ([EH89], [EH90]) puis par Hofer et Zendher ([HZ90], [HZ94]). Pour une partie $U$ incluse dans $\mathbb{R}^{2 n}$, Hofer avait prouvé l'inégalité

$$
e_{d_{\infty}}(U) \geq c_{H Z}(U)
$$

où $c_{H Z}$ désigne la capacité de Hofer-Zehnder (voir [Hof90]), démontrant ainsi la non-dégénérescence de la distance de Hofer dans le cas où $M=\mathbb{R}^{2 n}$.

Notons une conséquence immédiate de ces résultats, qui sera fondamentale dans la suite pour définir une notion d'homéomorphisme hamiltonien.

Corollaire 4.3.10. Soient $\left(H_{i}\right)_{i \in \mathbb{N}}, H$ des hamiltoniens lisses appartenant $\grave{a} \mathcal{H}$, et $\varphi$ un homéomorphisme. On fait les hypothèses suivantes :

(i) $\lim _{i \rightarrow+\infty}\left\|H_{i}-H\right\|=0$ pour la norme de Hofer dans $\mathcal{H}$;

(ii) $\lim _{i \rightarrow+\infty} d_{C^{0}}\left(\varphi_{H_{i}}^{1}, \varphi\right)=0$ pour la norme $C^{0}$. Alors $\varphi=\varphi_{H}^{1}$. 
Démonstration. Par l'absurde, supposons que $\varphi \neq \varphi_{H}^{1}$, et posons $\psi=$ $\varphi_{H}^{1} \varphi^{-1}$. Ainsi $\psi \neq 1$, donc on peut trouver une petite boule $U$ telle que $U \cap \psi(U)=\emptyset$. Puisque $\psi$ est limite uniforme de $\psi_{i}=\varphi_{H}^{1}\left(\varphi_{H_{i}}^{1}\right)^{-1}$, on a $U \cap \psi_{i}(U)=\emptyset$ pour $i$ suffisamment grand. On a donc d'une part

$$
d_{\infty}\left(1, \psi_{i}\right) \geq e_{d_{\infty}}(U)>0
$$

et d'autre part

$$
d_{\infty}\left(1, \psi_{i}\right) \leq\left\|H_{i}-H\right\|
$$

avec

$$
\lim _{i \rightarrow+\infty}\left\|H_{i}-H\right\|=0
$$

ce qui est absurde. Donc $\varphi=\varphi_{H}^{1}$.

Enfin terminons cette section par l'étude des pseudo-distances $d_{p}$, définies par

$$
d_{p}(1, \varphi)=\inf \left\{\int_{0}^{1}\left(\int_{M}|H|^{p} \omega\right)^{1 / p} d t \mid \varphi_{H}^{1}=\varphi, H \in \mathcal{H}\right\} .
$$

Théorème 4.3.11 (Eliashberg-Polterovich). Pour $p$ fini, la pseudodistance $d_{p}$ est dégénérée. De plus, si $M$ est fermée, alors $d_{p}$ est identiquement nulle.

Démonstration. ([Pol01]) Soit $U$ une boule suffisamment petite plongée dans $M$. En prenant des coordonnées symplectiques au voisinage de $U$, on peut facilement trouver un flot hamiltonien "horizontal" qui déplace $U$, i.e. il existe un hamiltonien autonome $H \in C^{\infty}(M)$ tel que $\varphi_{H}^{1}(U) \cap U=\emptyset$.

Posons $S_{t}=\varphi_{H}^{t}(\partial U)$, et soit $V_{t}$ un voisinage de $S_{t}$. A l'aide d'une fonction plateau, on construit $K \in C^{\infty}(M \times[0,1])$ qui vérifie

$$
K_{t}= \begin{cases}H & \operatorname{sur} S_{t} \\ 0 & \operatorname{sur} M \backslash V_{t} .\end{cases}
$$

Quitte à rajouter une constante $c_{t}$ pour normaliser, on suppose que $K \in \mathcal{H}$. Notons $\psi_{K}^{1}$ le difféomorphisme hamiltonien engendré par $K$. Puisque $K$ coïncide avec $H$ sur $S_{t}$, il est immédiat que $\psi_{K}^{1}(U) \cap U=\emptyset$. En prenant des voisinages $V_{t}$ arbitrairement petits, on peut s'assurer que la norme

$$
\|K\|_{p}=\int_{0}^{1}\left(\int_{V_{t}}\left|K_{t}\right|^{p} \omega\right)^{1 / p} d t
$$

soit aussi petite que l'on veut. Ainsi $e_{d_{p}}(U)=0$, ce qui implique que la distance $d_{p}$ est dégénérée. Enfin, si $M$ est fermée, la dernière conclusion résulte de la simplicité du groupe des difféomorphismes hamiltoniens. 
Remarque 4.3.12. Dans le cas du disque $\mathbb{D}^{2}$, ou plus généralement d'une variété symplectique non fermée, en notant

$$
G_{d_{p}}=\left\{\varphi \in \operatorname{Ham}\left(\mathbb{D}^{2}, \partial \mathbb{D}^{2}, \omega\right) \mid d_{p}(1, \varphi)=0\right\}
$$

le second théorème de Banyaga permet aussitôt de conclure que $G_{p}$ contient le noyau du morphisme de Calabi. Un autre résultat d'Eliashberg et Polterovich ([EP93]) nous donne la relation plus fine suivante:

$$
d_{p}(1, \varphi)=\operatorname{vol}(M)^{\frac{1-p}{p}}|\operatorname{Cal}(\varphi)|
$$

avec les conventions $(+\infty)^{0}=1$ et $(+\infty)^{-c}=0$.

\subsection{Approche de Oh}

Dans les deux premières sections de ce chapitre, on a tenté d'obtenir des résultats de simplicité concernant les groupes d'homéomorphismes préservant la mesure en essayant d'étendre à ces groupes les invariants de Calabi, initialement définis pour les difféomorphismes. Cette approche a fonctionné pour le premier invariant de Calabi (construction de Fathi) mais pas pour le second. De plus, la question de la simplicité du noyau de l'invariant de Fathi reste encore ouverte.

Dans cette section, on change d'approche et l'on essaie de définir un analogue $C^{0}$ au groupe des difféomorphismes hamiltoniens, selon des idées de Oh [OM07]. Pour que la comparaison soit acceptable, il faut que ce sous-groupe soit normal dans $\operatorname{Homeo}_{0}(M, \mu)$ et qu'il soit contenu dans le noyau de l'invariant de Fathi. Si c'est un sous-groupe propre, alors en particulier les groupes $\mathrm{Homeo}_{0}\left(\mathbb{S}^{2}, \mu\right)$ et $\mathrm{Homeo}_{0}\left(\mathbb{D}^{2}, \partial \mathbb{D}^{2}, \mu\right)$ ne sont pas simples. Sinon, tout revient à étudier la simplicité de ce groupe, et pour cela, on peut toujours essayer de définir un second invariant de Calabi.

Une première idée pour définir ce groupe est tout simplement de prendre l'adhérence de $\operatorname{Ham}(M, \omega)$ dans $\operatorname{Homeo}_{0}(M, \mu)$ pour la topologie $C^{0}$. Cependant, si l'on procède de la sorte on peut facilement obtenir tout le groupe $\operatorname{Homeo}_{0}(M, \mu)$. Par exemple, dans le cas de la sphère $\mathbb{S}^{2}$, on a $\operatorname{Ham}\left(\mathbb{S}^{2}, \omega\right)=\operatorname{Diff} f_{0}^{\infty}\left(\mathbb{S}^{2}, \omega\right)$ et l'on sait qu'en dimension deux, on peut approcher tout homéomorphisme préservant l'aire par un difféomorphisme préservant l'aire.

Une autre idée, motivée par la nature $C^{0}$ de la distance de Hofer, consisterait alors à compléter le groupe $\operatorname{Ham}(M, \omega)$ vis-à-vis de cette distance, i.e. à prendre des classes de suites de Cauchy d'éléments de $\operatorname{Ham}(M, \omega)$ pour la distance de Hofer. Le souci de taille auquel on est rapidement confronté est de ne pas pouvoir représenter chaque classe par un homéomorphisme, i.e. on sort du groupe $\operatorname{Homeo}(M, \mu)$ (voir [OM07]).

On va alors chercher un compromis entre ces deux idées. Au vu du résultat 4.3.10, une topologie "produit" entre la topologie $C^{0}$ et la topologie de Hofer sur $\operatorname{Ham}(M, \omega)$ pourrait donner un résultat intéressant. 
Cependant, pour définir correctement une notion d'homéomorphisme hamiltonien et en faire un groupe, il va d'abord falloir raisonner sur l'espace des isotopies hamiltoniennes pour définir des isotopies hamiltoniennes topologiques (il n'y a rien de surprenant, c'est de cette manière que l'on procède pour définir les difféomorphismes hamiltoniens). Commençons par préciser ce que l'on entend par topologie "produit" entre la topologie $C^{0}$ et la topologie de Hofer, et introduisons à cet effet la notion de distance hamiltonienne, que l'on doit à $\mathrm{Oh}$.

Définition 4.4.1. Soient $\Phi_{H}, \Psi_{K}$ deux isotopies hamiltoniennes engendrées par les fonctions $H, K \in \mathcal{H}$. On définit leur distance hamiltonienne par

$$
d_{H a m}\left(\Phi_{H}, \Psi_{K}\right)=\bar{d}\left(\Phi_{H}, \Psi_{K}\right)+\|H-K\|
$$

où $\bar{d}\left(\Phi_{H}, \Psi_{K}\right)=\sup _{t \in[0,1]} d_{C^{0}}\left(\varphi_{H}^{t}, \psi_{K}^{t}\right)$ et $\|$.$\| est la norme de Hofer sur$ $\mathcal{H}$.

Remarque 4.4.2. En prenant le temps 1 des isotopies, on définit de manière analogue une distance hamiltonienne sur le groupe $\operatorname{Ham}(M, \omega)$. On peut d'ailleurs montrer que muni de cette topologie, le groupe $\operatorname{Ham}(M, \omega)$ reste un groupe topologique connexe par arcs (voir [OMO7]).

On peut alors maintenant définir l'ensemble des isotopies hamiltoniennes topologiques, ou haméotopies, en complétant l'espace des isotopies hamiltoniennes (lisses) par rapport à la distance hamiltonienne, puis définir les homéomorphismes hamiltoniens, baptisés haméomorphismes, comme temps 1 d'haméotopies. Voici des définitions formelles.

Définition 4.4.3. Soit $\Phi=\left(\varphi^{t}\right)_{t \in[0,1]}$ une isotopie dans Homeo $(M)$. On dit que $\Phi$ est une haméotopie s'il existe une suite de hamiltoniens lisses $\left(H_{i}\right)_{i \in \mathbb{N}}$ dans $\mathcal{H}$ qui vérifie les deux conditions suivantes :

(i) $\lim _{i \rightarrow+\infty} \bar{d}\left(\Phi_{H_{i}}, \Phi\right)=0$;

(ii) $\lim _{i, j \rightarrow+\infty}\left\|H_{i}-H_{j}\right\|=0$.

Dans ce cas, $\varphi=\varphi^{1}$ est un haméomorphisme, et on note $\operatorname{Hameo}(M, \omega)$ l'ensemble des haméomorphismes.

Notons $L^{1, \infty}([0,1] \times M)$ le complété de $\mathcal{H}$ pour la norme de Hofer, i.e.

$$
L^{1, \infty}([0,1] \times M)=\{H:[0,1] \times M \longrightarrow \mathbb{R} \mid\|H\|<\infty\} .
$$

La suite $\left(H_{i}\right)_{i \in \mathbb{N}}$ dans la définition est de Cauchy pour la norme de Hofer sur $\mathcal{H}$, elle admet donc une limite $H$ appartenant à $L^{1, \infty}([0,1] \times M)$, non nécessairement lisse, qui joue le rôle de fonction hamiltonienne pour l'isotopie $\Phi$. En particulier, on a

$$
\lim _{i \rightarrow+\infty} d_{H a m}\left(\Phi_{H_{i}}, \Phi\right)=\lim _{i \rightarrow+\infty}\left(\bar{d}\left(\Phi_{H_{i}}, \Phi\right)+\left\|H_{i}-H\right\|\right)=0 .
$$


Remarque 4.4.4. On obtient automatiquement que $\Phi$ préserve la mesure car chaque $\varphi^{t}$ est une limite uniforme d'homéomorphismes préservant la mesure, donc préserve la mesure (voir [Fat80a]).

L'ensemble $\operatorname{Hameo}(M, \omega)$ est donc inclus dans le groupe $\operatorname{Homeo}(M, \mu)$. On le munit de la topologie induite, i.e. de la topologie $C^{0}$. Pour s'assurer que $\operatorname{Hameo}(M, \omega)$ est un bon analogue au groupe $\operatorname{Ham}(M, \omega)$, il faut avant tout vérifier que c'est un sous-groupe normal de $\operatorname{Homeo}_{0}(M, \mu)$. C'est ce que l'on fait dans la proposition suivante (voir [OM07]).

Théorème 4.4.5 (Oh-Müller). Hameo $(M, \omega)$ est un sous-groupe normal de $\operatorname{Homeo}(M, \mu)$, connexe par arcs.

Démonstration. Commençons par vérifier que $\operatorname{Hameo}(M, \omega)$ est stable par composition. Soient $\Phi=\left(\varphi^{t}\right)_{t \in[0,1]}$ et $\Psi=\left(\psi^{t}\right)_{t \in[0,1]}$ deux haméotopies, définies par des suites de hamiltoniens $\left(H_{i}\right)_{i \in \mathbb{N}}$ et $\left(K_{i}\right)_{i \in \mathbb{N}}$ qui vérifient

$$
\lim _{i \rightarrow+\infty} d_{\text {Ham }}\left(\Phi_{H_{i}}, \Phi\right)=0 \quad ; \quad \lim _{i \rightarrow+\infty} d_{\text {Ham }}\left(\Psi_{K_{i}}, \Psi\right)=0 .
$$

On veut prouver que

$$
\Phi . \Psi: t \in[0,1] \longmapsto \varphi^{t} \psi^{t} \in \operatorname{Homeo}(M, \mu)
$$

est aussi une haméotopie. Rappelons que l'isotopie $\Phi_{H_{i}} \cdot \Psi_{K_{i}}$ est engendrée par la fonction $H_{i} \# K_{i}$ et notons $H, K \in L^{1, \infty}([0,1] \times M)$ les limites des suites $\left(H_{i}\right)_{i \in \mathbb{N}}$ et $\left(K_{i}\right)_{i \in \mathbb{N}}$ pour la norme de Hofer. Il suffit alors de prouver que

$$
\begin{aligned}
\lim _{i \rightarrow+\infty} & d_{\text {Ham }}\left(\Phi_{H_{i}} \cdot \Psi_{K_{i}}, \Phi . \Psi\right) \\
\quad= & \lim _{i \rightarrow+\infty}\left(\bar{d}\left(\Phi_{H_{i}} \cdot \Psi_{K_{i}}, \Phi . \Psi\right)+\left\|H_{i} \# K_{i}-H \# K\right\|\right)=0 .
\end{aligned}
$$

Le premier membre de la somme tend clairement vers 0, car la composition est continue pour la topologie $C^{0}$ sur $\operatorname{Homeo}(M)$. Pour le second membre, on écrit

$$
\begin{aligned}
\left\|H_{i} \# K_{i}-H \# K\right\| \leq & \left\|H_{i}-H+K_{i}\left(\Phi_{H_{i}}\right)^{-1}-K \Phi^{-1}\right\| \\
\leq & \left\|H_{i}-H\right\|+\left\|K_{i}\left(\Phi_{H_{i}}\right)^{-1}-K \Phi^{-1}\right\| \\
\leq & \left\|H_{i}-H\right\|+\left\|K_{i}\left(\Phi_{H_{i}}\right)^{-1}-K_{i} \Phi^{-1}\right\| \\
& +\left\|K_{i} \Phi^{-1}-K \Phi^{-1}\right\| \\
\leq & \left\|H_{i}-H\right\|+\left\|K_{i}\left(\Phi_{H_{i}}\right)^{-1}-K_{i} \Phi^{-1}\right\|+\left\|K_{i}-K\right\| .
\end{aligned}
$$

Par hypothèses, les termes $\left\|H_{i}-H\right\|$ et $\left\|K_{i}-K\right\|$ tendent vers 0 quand $i$ tend vers l'infini. Seul le terme du milieu mérite plus d'explications. Fixons $\varepsilon>0$. On trouve alors un entier $i_{0} \in \mathbb{N}$ tel que

$$
\forall i \in \mathbb{N}, i \geq i_{0} \Longrightarrow\left\|K_{i}-K_{i_{0}}\right\|<\varepsilon / 3 \text {. }
$$


Par uniforme continuité de $K_{i_{0}}$, on peut trouver $\delta>0$ tel que

$$
\bar{d}\left(\Phi_{H_{i}}, \Phi\right)<\delta \Longrightarrow \sup _{(t, x)}\left|K_{i_{0}}\left(t,\left(\varphi_{H_{i}}^{t}\right)^{-1}(x)\right)-K_{i_{0}}\left(t,\left(\varphi^{t}\right)^{-1}(x)\right)\right|<\varepsilon / 6
$$

ce qui nous donne $\left\|K_{i_{0}}\left(\Phi_{H_{i}}\right)^{-1}-K_{i_{0}} \Phi^{-1}\right\|<\varepsilon / 3$.

Enfin, on applique l'inégalité triangulaire

$$
\begin{aligned}
\left\|K_{i}\left(\Phi_{H_{i}}\right)^{-1}-K_{i} \Phi^{-1}\right\| \leq & \left\|K_{i}\left(\Phi_{H_{i}}\right)^{-1}-K_{i_{0}}\left(\Phi_{H_{i}}\right)^{-1}\right\| \\
& +\left\|K_{i_{0}}\left(\Phi_{H_{i}}\right)^{-1}-K_{i_{0}} \Phi^{-1}\right\| \\
& +\left\|K_{i_{0}} \Phi^{-1}-K_{i} \Phi^{-1}\right\| \\
\leq & \left\|K_{i}-K_{i_{0}}\right\|+\left\|K_{i_{0}}\left(\Phi_{H_{i}}\right)^{-1}-K_{i_{0}} \Phi^{-1}\right\| \\
& +\left\|K_{i_{0}}-K_{i}\right\| \\
\leq & \varepsilon / 3+\varepsilon / 3+\varepsilon / 3 \\
\leq & \varepsilon .
\end{aligned}
$$

Par un raisonnement complètement analogue, on montre que $\operatorname{Hameo}(M, \omega)$ est stable par passage à l'inverse et par conjugaison. C'est donc un sousgroupe normal.

Il nous reste enfin à prouver que $\operatorname{Hameo}(M, \omega)$ est connexe par arcs pour la topologie $C^{0}$. Soit $\varphi$ un haméomorphisme temps 1 d'une haméotopie $\Phi=\left(\varphi^{t}\right)_{t \in[0,1]}$. Il faut démontrer que chaque $\varphi^{s}$, pour $0<s<1$, est un haméomorphisme, ce que l'on va faire en montrant que l'isotopie

$$
\Phi^{\prime}: t \in[0,1] \longmapsto \varphi^{s t} \in \operatorname{Homeo}(M)
$$

est une haméotopie. Pour cela, notons $\left(H_{i}\right)_{i \in \mathbb{N}}$ la suite de hamiltoniens lisses définissant $\Phi$ et considérons les reparamétrisations $\left(H_{i}^{a}\right)_{i \in \mathbb{N}}$ pour la fonction $a(t)=s t$. Pour tout entier $i$, l'isotopie hamiltonienne engendrée par $H_{i}^{a}$ est donc

$$
\Phi_{H_{i}^{a}}: t \in[0,1] \longmapsto \varphi_{H_{i}}^{s t} .
$$

Il suffit alors de montrer que

$$
\lim _{i \rightarrow+\infty} d_{H a m}\left(\Phi_{H_{i}^{a}}, \Phi^{\prime}\right)=0 .
$$

Encore une fois, la convergence $C^{0}$ est immédiate. Il reste donc à s'assurer que la suite $H_{i}^{a}$ est de Cauchy pour la norme de Hofer, ce qui résulte facilement de l'inégalité $\left\|H_{i}^{a}-H_{j}^{a}\right\| \leq\left\|H_{i}-H_{j}\right\|$.

Remarque 4.4.6. On s'intéresse ici principalement au groupe $\operatorname{Hameo}(M, \omega)$ en tant que sous-groupe de Homeo $(M, \mu)$, et donc muni de la topologie induite. Cependant, $\operatorname{Hameo}(M, \omega)$ apparaît naturellement avec une topologie intrinsèque, la topologie hamiltonienne. Dans ce cadre, on peut montrer que $\operatorname{Hameo}(M, \omega)$ reste un groupe topologique connexe par arcs, mais c'est plus difficile. 
Revenons à la définition des haméomorphismes et des haméotopies. Le choix de la norme $\|\|=.\|.\| \|_{1, \infty}$ est motivé dans [OM07] par la possibilité d'étendre à ce nouveau cadre certains invariants symplectiques. Dans la définition 4.4.3, on aurait pu remplacer cette norme par la norme

$$
\sup _{t \in[0,1]}\left(O s c\left(H_{t}\right)\right)
$$

ou tout simplement par la norme $C^{0}$. Ce choix, à priori plus contraignant (puisque cette dernière norme est plus grande que $\|\cdot\|_{1, \infty}$ ), nous donne la même notion d'haméomorphisme (voir [Mül07], c'est un analogue à la proposition 4.3.2). Cependant, cette approche présente plusieurs avantages : la vérification que $\operatorname{Hameo}(M, \omega)$ est un sous-groupe normal de $\operatorname{Homeo}_{0}(M, \mu)$ est immédiate (puisque les limites d'hamiltoniens pour la norme $\|.\|_{\infty}$ sont uniformément continues) mais plus important encore, on dispose du théorème d'unicité suivant (voir [Vit06]).

Théorème 4.4.7 (Viterbo). Soit $\left(H_{i}\right)_{i \in \mathbb{N}}$ une suite de hamiltoniens appartenant à $\mathcal{H}$ qui converge uniformément vers $H$. Si l'isotopie $\Phi_{i}=$ $\left(\varphi_{H_{i}}^{t}\right)_{t \in[0,1]}$ converge uniformément vers l'identité, alors $H=0$.

Rappelons que notre objectif est de comprendre la structure du groupe $\operatorname{Ker}(\mathcal{F})$, où

$$
\mathcal{F}: \operatorname{Homeo}(M, \mu) \longrightarrow H_{1}(M, \mathbb{R}) / \Gamma_{\mu} .
$$

On sait que $\mathcal{F}$ est continue, et que $\operatorname{Ham}(M, \omega)$ est inclus dans $\operatorname{Ker}(\mathcal{F})$. Puisque les haméomorphismes sont en particulier des limites uniformes de difféomorphismes hamiltoniens, la proposition suivante est immédiate.

Proposition 4.4.8. Les haméomorphismes sont inclus dans le noyau de l'invariant de Fathi.

Mais on fait encore face à la question suivante.

Question 5. $\operatorname{Hameo}(M, \omega)$ est-il un sous-groupe propre de $\operatorname{Ker}(\mathcal{F})$ ?

Selon Oh, la réponse à cette question devrait être positive. Voici un problème un peu plus concret (voir [OM07], [Oh06]). On munit le disque $\mathbb{D}^{2}$ de ses coordonnées polaires, et on définit, comme en 3.2.5, une application $\varphi \rho \operatorname{par}$

$$
\varphi_{\rho}:(r, \theta) \longmapsto(r, \theta+\rho(r)) .
$$

Cette fois-ci, on fait les hypothèses suivantes sur la fonction

$$
\rho:] 0,1] \longrightarrow[0,+\infty[
$$

(i) $\rho(r)=0$ pour $r \in[1-\varepsilon, 1]$;

(ii) $\rho^{\prime}(r)<0$ pour $\left.r \in\right] 0,1-\varepsilon[$. 
Il est clair que $\varphi_{\rho}$ est un homéomorphisme préservant la mesure associée à $\omega=r d r \wedge \theta$ et fixe un voisinage du bord (par $(i))$. C'est le temps 1 du flot $\left(\varphi_{t \rho}\right)_{t \in[0,1]}$. Cependant, $\varphi_{\rho}$ n'est pas lisse en 0 par la condition (ii). Pour un choix convenable de la fonction $\rho$, on va montrer que $\varphi_{\rho}$ est un haméomorphisme. Sur le disque épointé, $\varphi_{\rho}$ est un difféomorphisme hamiltonien engendré par la fonction

$$
H_{\rho}(r)=\int_{r}^{1} s \rho(s) d s
$$

mais au voisinage de 0 , cette intégrale peut fort bien diverger. Choisissons alors $\rho$ de telle sorte que

$$
\int_{0}^{1} s \rho(s) d s<\infty .
$$

La fonction $H_{\rho}$ est alors définie et continue sur le disque $\mathbb{D}^{2}$, et sa norme de Hofer n'est rien d'autre que sa valeur en 0 (qui est aussi la valeur de l'intégrale convergente). Par convolution, il est facile de construire une suite de fonctions lisses $\left(\rho_{n}\right)_{n \in \mathbb{N}}$ telle que pour tout entier $n, \varphi_{\rho_{n}}$ soit lisse en 0 et telle que

$$
\lim _{n \rightarrow \infty} d_{C^{0}}\left(\varphi_{\rho_{n}}, \varphi_{\rho}\right)=0 .
$$

Cette dernière condition implique aisément la convergence $C^{0}$ de l'isotopie $\left(\varphi_{t \rho_{n}}\right)_{t \in[0,1]}$ vers $\left(\varphi_{t \rho}\right)_{t \in[0,1]}$. De plus, puisque la norme de Hofer $\left\|H_{\rho}\right\|$ est finie, on a également

$$
\lim _{n \rightarrow \infty}\left\|H_{\rho_{n}}-H_{\rho}\right\|=0
$$

et ainsi $\varphi_{\rho}$ est un haméomorphisme, associé à la suite de hamiltoniens lisses $\left(H_{\rho_{n}}\right)_{n \in \mathbb{N}}$.

$\mathrm{Au}$ contraire, supposons maintenant que

$$
\int_{0}^{1} s \rho(s) d s=\infty
$$

pour une certaine fonction $\rho$. Si l'on parvient à montrer que $\varphi_{\rho}$ n'est plus un haméomorphisme, on montre alors que Hameo $\left(\mathbb{D}^{2}, \partial \mathbb{D}^{2}, \omega\right)$ est un sousgroupe propre de $\operatorname{Homeo}\left(\mathbb{D}^{2}, \partial \mathbb{D}^{2}, \mu\right)$. Mais cette question reste également ouverte, elle est encore liée à l'existence d'une extension du second invariant de Calabi au groupe $\operatorname{Hameo}\left(\mathbb{D}^{2}, \partial \mathbb{D}^{2}, \omega\right)$ (voir la tentative dans [Oh06]).

Dans tous les cas, la question suivante reste importante.

Question 6. Est-ce que le groupe $\operatorname{Hameo}(M, \omega)$ est simple?

Il est évident que pour pouvoir répondre à ces questions, il faut étudier plus en détails les propriétés intrinsèques de ce groupe d'homéomorphismes hamiltoniens. 


\section{Annexe A}

\section{Théorème de Schoenflies}

Dans cette annexe, on va énoncer quelques résultats fondamentaux sur la topologie des surfaces dont on fait usage dans le premier chapitre. Des références générales sont [Bre93], [Moi77] et [Bin83].

Commençons par rappeler la définition évidente suivante. On note $\mathbb{D}^{2}$ le disque unité fermé de $\mathbb{R}^{2}$ et $\mathbb{S}^{1}=\partial \mathbb{D}^{2}$ le cercle unité.

Définition A.0.9. On appelle disque fermé (resp. cercle) dans $M$ une partie homéomorphe à $\mathbb{D}^{2}$ (resp. $\mathbb{S}^{1}$ ).

Le premier résultat important est un théorème de Jordan. Il s'agit d'un exemple typique de résultat intuitif (le fait que lorsque l'on découpe la sphère le long d'une courbe fermée simple, on obtient deux morceaux) mais difficile à démontrer avec des outils élémentaires. Voici un énoncé précis.

Théorème A.0.10 (Jordan). Soit $S$ un cercle dans $\mathbb{S}^{2}$. Alors $S$ sépare $\mathbb{S}^{2}$ en deux composantes connexes.

On a un énoncé analogue si l'on remplace la sphère par le plan. Il existe de nombreuses preuves de ce théorème, mais la plus rapide utilise le langage de l'homologie. L'idée consiste à prouver que le groupe $H_{0}\left(\mathbb{S}^{2} \backslash S\right)$, qui "compte" le nombre de composantes connexes de $\mathbb{S}^{2} \backslash S$, est isomorphe à $\mathbb{Z}^{2}$, ce qu'il est facile de faire en utilisant une suite exacte de Mayer-Vietoris.

Il paraît aussi intuitif que si $S$ est un cercle de $\mathbb{S}^{2}$, alors les deux composantes connexes de $\mathbb{S}^{2} \backslash S$ sont homéomorphes à des disques ouverts. Encore une fois, la preuve est loin d'être évidente et résulte du théorème fondamental suivant. On identifie $\mathbb{S}^{1}$ avec son image par le plongement canonique $\mathbb{S}^{1} \hookrightarrow \mathbb{S}^{2}$.

Théorème A.0.11 (Schoenflies). Si S est un cercle dans $\mathbb{S}^{2}$, alors tout homéomorphisme $f: \mathbb{S}^{1} \rightarrow S$ préservant l'orientation s'étend en un homéomorphisme $\bar{f}$ de $\mathbb{S}^{2}$ préservant l'orientation. 


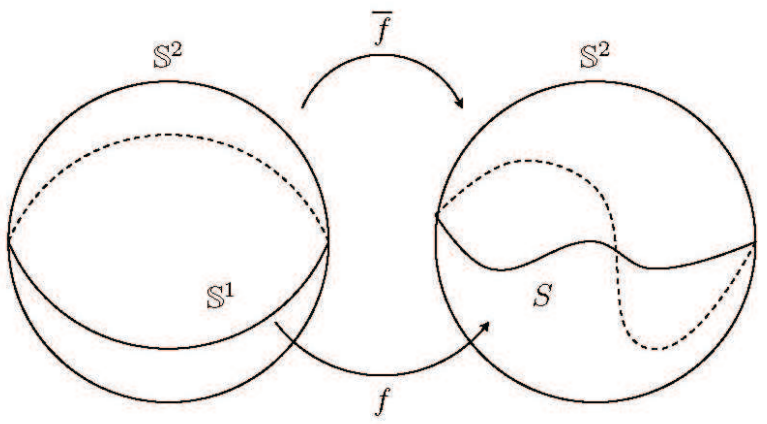

FIG. A.1 - Théorème de Schoenflies

En particulier, les composantes connexes de $\mathbb{S}^{2} \backslash S$ sont des disques car homéomorphes à celles de $\mathbb{S}^{2} \backslash \mathbb{S}^{1}$ (voir la figure A.1). On renvoie à [Moi77] ou [Bin83] pour des preuves purement topologiques. D'autres approches sont possibles, comme par exemple celles qui utilisent de l'analyse complexe ou bien encore une démonstration récente de Siebenmann ([Sie05]) basée sur des arguments de géométrie hyperbolique.

Contrairement au théorème de Jordan qui se généralise aussitôt en dimension plus grande, le théorème de Schoenflies est faux en dimension supérieure (comme en témoigne l'exemple classique de la sphère à cornes d'Alexander) à moins de rajouter une hypothèse de "platitude locale" : on demande que le plongement de $\mathbb{S}^{n-1}$ dans $\mathbb{S}^{n}$ s'étende en un plongement de $\mathbb{S}^{n-1} \times[-\varepsilon, \varepsilon]$ pour $\epsilon>0$ (on pourra consulter [Bre93] pour une preuve).

On utilisera souvent le théorème de Schoenflies pour étendre un plongement du cercle $\mathbb{S}^{1}=\partial \mathbb{D}^{2}$ en un plongement du disque $\mathbb{D}^{2}$. Dans cette optique, on aura également besoin d'une version à support compact (voir [Bin83] par exemple).

Théorème A.0.12. Soient $D, D^{\prime}$ deux disques fermés du plan $\mathbb{R}^{2}$. Alors tout homéomorphisme entre $D$ et $D^{\prime}$ s'étend en homéomorphisme du plan à support compact.

Remarque A.0.13. Lorsque $D$ et $D^{\prime}$ sont des disques euclidiens, on peut construire explicitement un tel homéomorphisme. En effet, on peut supposer que ces disques sont centrés en 0 , et que $D$ est inclus dans $D^{\prime}$ : par exemple, il suffit de les translaté en appliquant le temps 1 d'un champ de vecteurs constant multiplié par une fonction plateau convenable. Choisissons alors un disque euclidien $D^{\prime \prime}$ (également centré en 0) contenant 
$D^{\prime}$. On déforme radialement $D$ en $D^{\prime}$, puis on ralentit progressivement entre $D^{\prime}$ et $D^{\prime \prime}$ jusqu'à obtenir l'identité à partir de $D^{\prime \prime}$. On a bien un homéomorphisme qui envoie $D$ dans $D^{\prime}$ et qui est à support dans $D^{\prime \prime}$.

Le théorème de Schoenflies "à support compact" est également valable en dimension plus grande que deux si l'on rajoute l'hypothèse de platitude locale. Ce résultat, connu sous le nom de l'Annulus Conjecture, a longtemps été un problème ouvert et sa preuve complète est très difficile (spécialement en dimension quatre).

Enfin, on a également besoin d'une dernière amélioration du théorème de Schoenflies (une version "à paramètres"). On veut pouvoir contrôler l'extension fournie par le théorème, en particulier obtenir une extension proche de l'identité si l'homéomorphisme initial l'est. Une preuve du résultat suivant, valable en toute dimension, se trouve par exemple dans [EK71].

Théorème A.0.14. Soient $S_{1}$ et $S_{2}$ deux cercles centrés en 0 dans $\mathbb{R}^{2}$ bordant des disques euclidiens $D_{1}$ et $D_{2}$. Alors pour tout $r>0$, il existe $s>0$ tel que tout plongement $f: D_{1} \hookrightarrow D_{2}$ avec $d\left(f, 1_{D_{1}}\right)<s$ s'étend en un homéomorphisme $\vec{f}: D_{2} \rightarrow D_{2}$ avec $\bar{f}_{\mid S_{2}}=1$ et $d\left(\bar{f}, 1_{D_{2}}\right)<r$. 


\section{Annexe B}

\section{Théorème d'Epstein}

Dans cette annexe, on souhaite donner une preuve d'un théorème d'Epstein ([Eps70]). Il affirme que si un groupe d'homéomorphismes possède de bonnes propriétés de transitivité et de fragmentation, alors son sousgroupe des commutateurs est simple. Un tel groupe est donc simple si et seulement si il est parfait.

On se donne $X$ un espace topologique séparé paracompact, $G$ un groupe d'homéomorphismes de $X$ et $\mathcal{O}$ une base d'ouverts de $X$.

Définition B.0.15. On dit que $(X, G, \mathcal{O})$ vérifie les axiomes d'Epstein si et seulement si :

(E1) pour tout $U \in O$ et $g \in G$, on a $g U \in \mathcal{O}$;

(E2) $G$ agit transitivement sur $\mathcal{O}:$ pour tout $U, U^{\prime} \in \mathcal{O}$, il existe $g \in G$ tel que $g(U)=U^{\prime}$;

(E3) soient $g \in G, U \in \mathcal{O}$ et $\mathcal{U} \subseteq \mathcal{O}$ un recouvrement de $X$, alors il existe un entier $n$, des éléments $g_{1}, \ldots, g_{n} \in G$ et $V_{1}, \ldots, V_{n} \in \mathcal{U}$ tels que $g=g_{n} \ldots g_{1}, \operatorname{supp}\left(g_{i}\right) \subseteq V_{i}$ pour $1 \leq i \leq n$ et

$$
\operatorname{supp}\left(g_{i}\right) \cup\left(g_{i-1} \ldots g_{1} \bar{U}\right) \neq X .
$$

Théorème B.0.16 (Epstein). Si $(X, G, \mathcal{O})$ vérifie les axiomes d'Epstein, alors le sous-groupe des commutateurs de $G$ est simple. En particulier, $G$ est simple si et seulement si il est parfait.

On note $[G, G]$ le sous-groupe engendré par les commutateurs de $G$. Ce résultat est une conséquence immédiate de l'énoncé un peu plus général suivant.

Théorème B.0.17. Si $(X, G, \mathcal{O})$ vérifie les axiomes d'Epstein, et si $N$ est un sous-groupe non trivial de $G$ normalisé par $[G, G]$ (au sens où si $n \in N$ et $g \in[G, G]$, alors $[n, g] \in N)$, alors $[G, G]$ est inclus dans $N$. 
Démonstration. On suppose $G$ non trivial et $X$ connexe (la connexité résulte en fait des axiomes). La preuve se découpe en plusieurs étapes.

(a) Si $h \in G$ est à support dans $V \in \mathcal{O}$, alors il existe $f \in[G, G]$ tel que $f_{\mid V}=h_{\mid V}$.

Soit $W \in \mathcal{O}$ disjoint de $V$. Par (E2), on trouve $g \in G$ tel que $g W=V$. Posons $f=[g, h]=g^{-1} h^{-1} g h$. Il apparaît que $f$ est un produit de deux homéomorphismes à support disjoints, à savoir $h$ à support dans $V$ et $g^{-1} h^{-1} g$ à support dans $g^{-1} V=W$. La conclusion est alors immédiate.

(b) $[G, G]$ agit transitivement sur $\mathcal{O}$.

Soient $U$ et $U_{1}$ des éléments de $\mathcal{O}$ et $g \in G$ tel que $g U=U_{1}$. On utilise l'axiome (E3) : on trouve un entier $n, h_{1}, \ldots, h_{n} \in G$ et $V_{1}, \ldots, V_{n} \in \mathcal{O}$ tels que $g=h_{n} \ldots h_{1}, \operatorname{supp}\left(h_{i}\right) \subseteq V_{i}$ et pour $1 \leq i \leq n$

$$
K_{i}=\operatorname{supp}\left(h_{i}\right) \cup\left(h_{i-1} \ldots h_{1} \bar{U}\right) \neq X .
$$

Puisque $X-K_{i}$ est non vide, on trouve $W_{i} \subseteq X-K_{i}$ et $g_{i} \in G$ tels que $g_{i} W_{i}=V_{i}$. Pour $1 \leq i \leq n$, posons $f_{i}=\left[g_{i}, h_{i}\right]$ et $f=f_{n} \ldots f_{1} \in[G, G]$. L'étape (a) nous donne $f_{i \mid h_{i-1} \ldots h_{1} \bar{U}}=h_{i \mid h_{i-1} \ldots h_{1} \bar{U}}$ puisque $g_{i}^{-1}\left(\operatorname{supp}\left(h_{i}\right)\right)$ et $h_{i-1} \ldots h_{1} \bar{U}$ sont disjoints (leur intersection est contenue dans $W_{i} \cap K_{i}=\emptyset$ ). On obtient alors de manière inductive

$$
f U=f_{n} \ldots f_{1} U=h_{n} \ldots h_{1} U=g U=U_{1}
$$

ce qui prouve la transitivité de $[G, G]$.

(c) Si $h \in G$ est à support dans $V \in \mathcal{O}$, alors il existe $\rho \in N$ tel que $\rho_{\mid V}=h_{\mid V}$.

Commençons par établir cette propriété pour un ouvert $V$ bien choisi. Soit $\alpha \in N$ différent de l'identité. Prenons $x \in X$ tel que $\alpha(x) \neq x$ et $U \in \mathcal{O}$ un voisinage de $x$ qui vérifie encore $U \cap \alpha^{-1} U=\emptyset$. Soient $V$ et $W$ des éléments de $\mathcal{O}$, avec $x \in V, V \cap W=\emptyset$ et $\bar{V} \cup \bar{W} \subseteq U$. Soient enfin $g \in G$ vérifiant $g W=V$ et $h \in G$ à support dans $V$. Posons $\rho=$ $[\alpha,[g, h]]$ : un raisonnement analogue à (a) permet de conclure que $\rho_{\mid V}=$ $h_{\mid V}$. Maintenant, en utilisant (b) et en conjuguant $\rho$ par un élément de $[G, G]$, on peut choisir un ouvert $V$ arbitraire, un élément $h \in G$ à support dans $V$ et trouver $\rho \in N$ (rappelons que $N$ est normalisé par $[G, G]$ ) tel que $\rho_{\mid V}=h_{\mid V}$.

(d) Les orbites de l'action de $N$ sur $X$ sont denses.

En fait, on va prouver que les orbites de $N$ coïncident avec celles de $G$, qui sont denses par l'axiome (E2). Soient $x, y \in X$ et $g \in G$ tels que $y=g(x)$. Par (E3), on écrit $g=g_{n} \ldots g_{1}$ avec $g_{i} \in G$ à support dans $V_{i} \in \mathcal{O}$. Choisissons alors $g$ de telle sorte que l'entier $n$ dans sa décomposition soit minimal, alors pour $1 \leq i \leq n, g_{i-1} \ldots g_{1}(x) \in V_{i}\left(\right.$ car sinon $g^{\prime}=g_{1} \ldots g_{i-1} g_{i+1} \ldots g_{n}$ 
enverrait $x$ sur $y$ et contredirait l'hypothèse de minimalité). En utilisant (c), on trouve $\rho_{i} \in N$ qui coïncide avec $g_{i}$ sur $V_{i}$, pour $1 \leq i \leq n$. On obtient alors

$$
y=g_{n} \ldots g_{1}(x)=\rho_{n} \ldots \rho_{1}(x)
$$

ce qui prouve que les orbites de $N$ sont celles de $G$.

(e) Si $h_{1}, h_{2} \in G$ sont à support dans $V \in \mathcal{O}$, alors $\left[h_{1}, h_{2}\right] \in N$.

Par un argument analogue à la conclusion de $(c)$, on se réserve le droit de montrer ceci pour un $V \in \mathcal{O}$ bien choisi. Fixons $x$ dans $X$, on peut alors trouver par $(d)$ des éléments $\alpha_{1}, \alpha_{2}$ dans $N$ tels que $x, \alpha_{1}(x)$ et $\alpha_{2}(x)$ soient distincts. Par continuité, on se donne $U \in \mathcal{O}$ un voisinage de $x$ tel que $U, \alpha_{1} U$ et $\alpha_{2} U$ soient disjoints. Par transitivité de $G$, on peut trouver $g_{1}, g_{2} \in G$ suffisamment petits pour que $x, g_{1}(x)$ et $g_{2}(x)$ soient des éléments distincts de $U$. Encore une fois, on se donne $V \in \mathcal{O}$ un voisinage de $x$ tel que $V, g_{1} V$ et $g_{2} V$ soient des parties disjointes de $U$. On applique alors (c) pour obtenir $\rho_{i} \in N$, en utilisant $\alpha_{i}, g_{i}, W_{i}=g_{i}^{-1} V$ et un $h_{i} \in G$ arbitraire à support dans $V$, ceci pour $i=1,2$. On vérifie que $\rho_{1}$ est à support dans les ensembles $V, g_{1}^{-1} V, \alpha_{1}^{-1} V$ et $\alpha_{1}^{-1} g_{1}^{-1} V$ et que $\rho_{2}$ est à support dans les ensembles $V, g_{2}^{-1} V, \alpha_{2}^{-1} V$ et $\alpha_{2}^{-1} g_{2}^{-1} V$. Par notre construction, ces ensembles sont tous disjoints, ce qui donne aussitôt $\left[h_{1}, h_{2}\right]=\left[\rho_{1}, \rho_{2}\right] \in N$.

(f) G est engendré par les $\left(h_{i}\right)_{i \in I}$ tels que $\left[h_{i}, h_{j}\right] \in N$, pour tout $i, j \in I$.

Puisque $X$ est paracompact, on peut trouver un recouvrement $\mathcal{U} \subseteq \mathcal{O}$ qui possède la propriété suivante : si $V_{1}, V_{2} \in \mathcal{U}$ et $V_{1} \cap V_{2} \neq \emptyset$, alors il existe $U \in \mathcal{O}$ tel que $V_{1} \cup V_{2} \subseteq U$. Considérons l'ensemble

$$
A=\{h \in G \mid \exists W \in \mathcal{U} \text { tel que } \operatorname{supp}(h) \subseteq W\} .
$$

Par (E3), $A$ engendre $G$. Soient $h_{1}, h_{2} \in A$, à support respectivement dans $W_{1}$ et $W_{2}$. Alors ou bien $W_{1}$ et $W_{2}$ sont disjoints, auquel cas $\left[h_{1}, h_{2}\right]=1 \epsilon$ $N$ ou bien $W_{1} \cap W_{2}$ est non vide, alors $h_{1}$ et $h_{2}$ sont à support dans un élément $U \in \mathcal{O}$ et $(e)$ nous assure que $\left[h_{1}, h_{2}\right] \in N$.

(g) Si $h_{1}, h_{2} \in A$ et $g \in G$, alors $g\left[h_{1}, h_{2}\right] g^{-1} \in N$.

On se donne $W_{i} \in \mathcal{U}$ contenant le support de $h_{i}$ pour $i=1,2$. On suppose que $W_{1} \cap W_{2}$ est non vide sinon le résultat est évident. Soit alors $U \in \mathcal{O}$ tel que $W_{1} \cup W_{2} \subseteq U$. Appliquons (E3) à $g \in G$ et $U \in \mathcal{O}$ : il existe un entier $n$, des éléments $g_{1}, \ldots, g_{n} \in G$ et $V_{1}, \ldots, V_{n} \in \mathcal{U}$ tels que $g=g_{n} \ldots g_{1}$, $\operatorname{supp}\left(g_{i}\right) \subseteq V_{i}$ et pour $1 \leq i \leq n$

$$
K_{i}=\operatorname{supp}\left(g_{i}\right) \cup\left(g_{i-1} \ldots g_{1} \bar{U}\right) \neq X .
$$

Soient $U_{i} \in \mathcal{O}$ dans le complémentaire de $K_{i}$, et $\beta_{i} \in G$ tels que $\beta_{i} U_{i}=V_{i}$. Posons enfin $\gamma_{i}=\left[\beta_{i}, g_{i}\right]$ et $\gamma=\gamma_{n} \ldots \gamma_{1}$. Pour $1 \leq i \leq n$, on a la relation

$$
g_{i} \ldots g_{1}\left[h_{1}, h_{2}\right] g_{1}^{-1} \ldots g_{i}^{-1}=\gamma_{i} \ldots \gamma_{1}\left[h_{1}, h_{2}\right] \gamma_{1}^{-1} \gamma_{i}^{-1}
$$


qui se démontre (de manière inductive) en remarquant que l'action de $\gamma_{i}$ coïncide avec celle de $g_{i}$ hors de $U_{i}$. On en déduit l'égalité

$$
g\left[h_{1}, h_{2}\right] g^{-1}=\gamma\left[h_{1}, h_{2}\right] \gamma^{-1}
$$

et puisque $\left[h_{1}, h_{2}\right] \in N, \gamma \in[G, G]$ et que $N$ est normalisé par $[G, G]$, on obtient bien que $g\left[h_{1}, h_{2}\right] g^{-1} \in N$.

(h) Fin de la preuve

Soit $A$ l'ensemble des générateurs défini plus haut. Notons $S$ le sousgroupe normal de $G$ engendré par l'ensemble des commutateurs d'éléments de $A$, i.e. $[A, A]$. D'après $(g), S$ est inclus dans $N$. Maintenant $A$ engendre $G$, donc $A S$ engendre le quotient $G / S$. Il apparaît alors que les éléments de $A S$ commutent entre eux, donc $G / S$ est abélien ce qui implique que $[G, G]$ est inclus dans $S$. Finalement, on a $[G, G] \subseteq N$, c'est ce qu'il fallait démontrer.

Voici la principale application du théorème d'Epstein.

Corollaire B.0.18. Soient $M$ une variété lisse et Dif $f_{0, c}^{r}(M)$ le groupe des difféomorphismes de $M$ de classe $C^{r}$ compactement $C^{r}$-isotopes à l'identité, pour $r \in \mathbb{N}^{*} \cup\{\infty\}$. Alors $\left[\right.$ Dif $f_{0, c}^{r}(M)$, Dif $\left.f_{0, c}^{r}(M)\right]$ est simple.

Démonstration. Pour simplifier un peu la situation, on va supposer que notre variété $M$ est compacte. Soit $\mathcal{O}$ l'ensemble des boules $C^{r}$-plongées dans $M$, i.e. les ouverts de la forme $\varphi B$, où $\varphi: \mathbb{R}^{n} \rightarrow M$ est un plongement de classe $C^{r}$ et $B$ la boule unité de $\mathbb{R}^{n}$. Il suffit alors de vérifier que $\left(M, \operatorname{Dif} f_{0}^{r}(M), \mathcal{O}\right)$ satisfait aux axiomes d'Epstein. Notons $G=\operatorname{Dif} f_{0}^{r}(M)$.

Le premier axiome (E1), à savoir que $G$ agit sur $\mathcal{O}$, est évident. Il faut ensuite prouver que $G$ agit transitivement sur $\mathcal{O}$. Soient $B$ et $B^{\prime}$ deux boules plongées dans $M$, et on cherche $\varphi \in G$ tel que $\varphi(B)=B^{\prime}$. Puisque $G$ agit transitivement sur $M(2.2 .4)$, on peut se ramener à la situation suivante : étant données $B$ la boule unité de $\mathbb{R}^{n}$ et $B^{\prime} \subseteq \mathbb{R}^{n}$ l'image de $B$ par un plongement $\varphi$ de classe $C^{r}$ qui fixe 0 , on souhaite trouver un difféomorphisme de $\mathbb{R}^{n}$ à support compact qui envoie $B$ sur $B^{\prime}$. Voici comment on peut procéder. Quitte à composer par une réflexion, on suppose que notre plongement $\varphi$ préserve l'orientation, on cherche alors à étendre $\varphi$ en un difféomorphisme de $\mathbb{R}^{n}$ à support compact. On obtient une isotopie $\left(\varphi^{t}\right)_{t \in[0,1]}$ avec $\varphi^{0}=1$ et $\varphi^{1}=\varphi$, en concaténant un chemin entre l'identité et $D_{0} \varphi$ (dans l'ouvert connexe $G L^{+}(n, \mathbb{R})$ ) avec le chemin

$$
t \in[0,1] \longrightarrow \begin{cases}\frac{1}{t} \varphi(t x) & \text { si } t \neq 0 \\ D_{0} \varphi & \text { si } t=0\end{cases}
$$

qui consiste à "aplatir" $\varphi$ sur sa différentielle à l'origine. Cette isotopie est de classe $C^{r-1}$, mais par des méthodes d'approximation (voir [Hir76] 
par exemple), on peut la supposer de classe $C^{r}$. On utilise maintenant la procédure d'extension des isotopies : après dérivation de l'isotopie on obtient un champ de vecteurs (non autonome) $X_{t}$ pour $t \in[0,1]$ qui n'est défini que sur le support de l'isotopie, mais par les techniques habituelles (i.e. des fonctions plateaux et un voisinage tubulaire), on l'étend en un champ de vecteurs $\tilde{X}_{t}$ défini sur $\mathbb{R}^{n}$ et à support compact. Enfin, on intègre ce champ de vecteurs pour obtenir une isotopie de $\mathbb{R}^{n}$ à support compact dont le temps 1 (que l'on suppose de classe $C^{r}$ par approximation) nous donne le difféomorphisme voulu. Ceci prouve l'axiome (E2).

Il nous reste enfin à prouver l'axiome (E3). On a déja démontré la propriété de fragmentation (lemme 2.2.1, et voir la remarque 2.2.3), donc il nous reste à prouver la condition supplémentaire. On commence par se donner une décomposition $g=g_{n} \ldots g_{1}$ avec des éléments $g_{i}$ à support dans des boules plongées pour $1 \leq i \leq n$. Si l'axiome (E3) n'est pas vérifié, il suffit d'appliquer le lemme suivant à chaque $g_{i}$ pour obtenir la propriété voulue.

Lemme B.0.19. Soient $g \in G$ à support dans une boule $V$, et $U$ un ouvert tel que $\bar{U} \neq X$. Alors on peut écrire $g=g_{2} g_{1}$ avec pour $j=1,2, g_{j} \in G \grave{a}$ support dans $V$ et tels que

$$
\operatorname{supp}\left(g_{1}\right) \cup \bar{U} \neq X \text { et } \operatorname{supp}\left(g_{2}\right) \cup g_{1} \bar{U} \neq X .
$$

Démonstration. Si le support de $g$ est dans $\bar{U}$, il suffit de prendre $g_{1}=1$ et $g_{2}=g$. Sinon, soient $x_{1} \notin \bar{U}$ tel que $g x_{1} \neq x_{1}$ et $x_{2} \notin \bar{U}$ distinct de $x_{1}$ et $g x_{1}$. Donnons-nous des petits voisinages $N_{1}$ de $x_{1}$ et $N_{2}$ de $x_{2}$ dans $X \backslash U$. En utilisant des fonctions plateaux, on peut construire $g_{1} \in G$ à support dans $V$, qui coïncide avec $g$ sur $N_{1}$ et qui est l'identité sur $N_{2}$. Posons alors $g_{2}=g\left(g_{1}\right)^{-1}$. On a évidemment $g=g_{2} g_{1}$ avec $g_{1}, g_{2} \in G$ à support dans $V$. De plus, puisque $x_{2} \notin \operatorname{supp}\left(g_{1}\right) \cup \bar{U}$, on a $\operatorname{supp}\left(g_{1}\right) \cup \bar{U} \neq X$. Enfin, étant donné que $g_{1 \mid N_{1}}=g, g_{2 \mid g_{1}\left(N_{1}\right)}=1$, donc $g_{1}\left(x_{1}\right) \notin \operatorname{supp}\left(g_{2}\right)$ et puisque $g_{1}\left(x_{1}\right) \notin g_{1}(\bar{U}), \operatorname{supp}\left(g_{2}\right) \cup g_{1} \bar{U} \neq X$. Ceci termine la preuve du lemme.

Le triplet $(M, G, \mathcal{O})$ satisfait donc aux axiomes d'Epstein, il s'ensuit que $[G, G]$ est simple.

Pour conclure, signalons que l'on peut également appliquer le théorème d'Epstein au groupe des homéomorphismes d'une variété topologique, en utilisant le résultat de fragmentation de [EK71] et en remplaçant l'utilisation de fonctions plateaux par le théorème de Schoenflies à support compact.

En revanche, les techniques d'Epstein ne sont pas directement applicables aux groupes conservatifs (par préservation d'une mesure ou d'un volume, l'axiome $(E 2)$ ne peut pas être vérifié), mais il est néanmoins possible d'adapter les arguments (voir [Fat80a] pour un exemple). 


\section{Annexe C}

\section{Théorème de}

\section{Nash-Moser-Hamilton}

Le théorème d'inversion locale dans les espaces de Banach affirme que si une application de classe $C^{1}$ a une différentielle inversible en un point, alors cette application est un difféomorphisme local au voisinage de ce point.

Ce résultat n'est plus valable si l'on sort du cadre des espaces de Banach. En effet, si M est une variété compacte, l'application exponentielle (au sens des groupes de Lie)

$$
\exp : \Gamma^{\infty}(\mathrm{T} M) \longrightarrow \operatorname{Diff} f^{\infty}(M)
$$

qui à un champ de vecteurs associe le temps 1 de son flot, a toujours une différentielle qui vaut l'identité en 0 . Cependant, elle n'est en général pas inversible au voisinage de 0 , car il existe des difféomorphismes arbitrairement proches de l'identité qui ne sont pas le temps 1 d'un flot. Voici un exemple simple pour s'en convaincre.

Soit $v \in \Gamma^{\infty}(\mathrm{TT})=C^{\infty}(\mathbb{T})$ un champ de vecteurs sans zéro sur le cercle, alors le temps 1 de son flot est un difféomorphisme $\varphi \in \operatorname{Diff}_{0}^{\infty}(\mathbb{T})$ sans point fixe qui de plus est nécessairement $C^{\infty}$-conjugué à une rotation : en effet, si $\theta$ paramètre $\mathbb{T}$, on peut faire un changement de coordonnées

$$
\tilde{\theta}=c \int \frac{d \theta}{v(\theta)} \operatorname{avec} c=\left(\int_{0}^{2 \pi} \frac{d \theta}{v(\theta)}\right)^{-1}
$$

de tel sorte que $v(\tilde{\theta})$ soit le champ de vecteurs constant égal à $c$ et son temps 1 la rotation d'angle $c$. Cependant on peut construire des difféomorphismes sans point fixe arbitrairement proches de l'identité qui ne sont pas $C^{\infty}$ conjuguée à une rotation : pour $n$ suffisamment grand et $\varepsilon$ suffisamment petit, $0<\varepsilon<1 / n$, le difféomorphisme

$$
f: \theta \longmapsto \theta+\pi / n+\varepsilon \sin ^{2}(n \theta) \quad \bmod 2 \pi
$$


est proche de l'identité, n'a pas de point fixe mais n'est pas conjugué à une rotation car il a une orbite périodique (le cycle $\{k \pi / n \mid 0 \leq k \leq 2 n-1\}$ ) et les autres orbites sont non périodiques. Ceci prouve que l'exponentielle n'est pas localement surjective (on peut aussi montrer sur des exemples qu'elle n'est pas localement injective).

Remarque C.0.20. Ainsi un difféomorphisme proche de l'identité peut ne pas être l'exponentielle d'un champ de vecteurs. Dans l'exemple précédent, on peut même montrer que $f$ ne possède pas de racine carrée, i.e elle ne peut pas s'écrire sous la forme $g^{2}$ pour un difféomorphisme $g$ (le temps 1 d'un flot vérifie cette propriété en prenant pour g le temps $1 / 2$ du flot). En revanche, de manière générale on peut toujours écrire

$$
f=\exp \left(v_{1}\right) \ldots \exp \left(v_{n}\right)
$$

pour des champs de vecteurs $v_{1}, \ldots, v_{n}$. En effet, les difféomorphismes qui s'écrivent de la sorte forment un sous-groupe normal non trivial du groupe des difféomorphismes $C^{\infty}$ isotopes à l'identité, qui d'après le théorème d'Epstein-Herman-Thurston-Mather, est simple.

Le but de cette annexe est donc d'énoncer un théorème d'inversion locale dans des espaces vectoriels plus généraux que les espaces de Banach mais sous des hypothèses plus restrictives : typiquement, on demandera de pouvoir résoudre l'équation linéaire sur tout un voisinage (c'est ce qui tombe en défaut dans l'exemple précédent). La preuve de ce théorème repose sur un algorithme de Newton dont l'idée est due à Nash ([Nas56]) et Moser ([Mos66]), mais on suivra le formalisme de Hamilton ([Ham82]). On pourra également consulter les références [Bos86] et [Féj04] pour voir des applications de ce théorème aux systèmes dynamiques.

Commençons par préciser quel type d'espace vectoriel on va considérer. Notre intérêt est de disposer d'un théorème d'inversion locale applicable aux problèmes dits de "petits diviseurs" qui apparaissent en dynamique : une fois l'équation linéarisée, on se retrouve avec un opérateur entre des espaces de fonctions qui cause une "perte de dérivées", i.e. il envoie une certaine classe de régularité (par exemple $C^{r}$ ) dans une classe de régularité plus faible $\left(C^{s}\right.$ pour $\left.s<r\right)$. Il est alors naturel de s'intéresser à l'espace obtenu en prenant toutes les classes de régularité possibles, mais commençons par une définition plus abstraite.

Définition C.0.21. Un espace de Fréchet est un espace vectoriel topologique localement convexe, métrisable et complet.

Il n'est pas difficile de vérifier que la convexité locale de $E$ est équivalente au fait que la topologie soit définie par une famille de semi-normes, et le fait que cette famille de semi-normes soit dénombrable est équivalent à la métrisabilité de $E$ (voir par exemple [Rud91]). Soit donc $\|.\|_{i \in \mathbb{N}}$ une famille 
dénombrable de semi-normes, indexée par $\mathbb{N}$ et que l'on peut supposer croissante (i.e. $\|.\|_{i} \leq\|.\|_{i+1}$ pour tout entier $i$ ). Une graduation est un choix d'une telle famille de semi-normes. Il peut y avoir plusieurs graduations possibles sur un espace de Fréchet, c'est pour cela qu'on introduit la définition évidente suivante.

Définition C.0.22. Un espace de Fréchet gradué est un espace de Fréchet muni d'une graduation.

L'archétype d'espace de Fréchet gradué s'obtient en prenant la limite d'une suite décroissante d'espaces de Banach $\left(E_{i},\|.\|_{i}\right)_{i \in \mathbb{N}}$, i.e.

$$
E=\bigcap_{i \in \mathbb{N}} E_{i}
$$

avec $E_{i+1}$ qui s'injecte continûment dans $E_{i}$. Ainsi, l'espace $C^{\infty}(M)$ des fonctions infiniment différentiables sur une variété compacte $M$, muni de la graduation "canonique" $\left(\|.\|_{C^{i}}\right)_{i \in \mathbb{N}}$, ou de manière plus générale l'espace des sections lisses $\Gamma^{\infty}(E)$ d'un fibré vectoriel $E$ au dessus de $M$, sont des espaces de Fréchet gradués.

Le cadre est encore trop général pour obtenir le résultat escompté. Il faut se restreindre à une catégorie de "bons" espaces de Fréchet dont les semi-normes vérifient en particulier des inégalités d'interpolations.

Définition C.0.23. Un bon espace de Fréchet est un espace de Fréchet gradué muni d'une famille d'applications linéaires continues, $S_{t}: E \rightarrow E$, $t>1$ appelés opérateurs de lissage, qui vérifie les propriétés suivantes : il existe $C_{j, k}>0$ tels que pour tous $x \in E, t>1$ et $j<k$, on ait

$$
\left\{\begin{array}{rr}
\left\|S_{t} x\right\|_{k} \leq C_{k, j} t^{k-j}\|x\|_{j} & \text { (régularisation) } \\
\left\|\left(1-S_{t}\right) x\right\|_{j} \leq C_{j, k} t^{j-k}\|x\|_{k} & \text { (approximation de l'identité). }
\end{array}\right.
$$

Si $E$ est un bon espace de Fréchet, on a alors les inégalités suivantes (dites inégalités de Hadamard)

$$
\|x\|_{l} \leq c_{k, n}\|x\|_{k}^{1-\alpha}\|x\|_{n}^{\alpha}
$$

pour tous $x \in E, k \leq l \leq n$ avec $l=(1-\alpha) k+\alpha n$ et des constantes $c_{k, n}$.

Exemple C.0.24. Soit $E$ un fibré vectoriel de dimension finie sur une variété compacte $M$. L'espace des sections lisses $\Gamma^{\infty}(E)$ muni de sa graduation canonique est alors un bon espace de Fréchet. On pourra consulter [Ham82] pour une preuve générale. Dans le cas particulier (qui nous intéresse) où E est l'espace des champs de vecteurs sur le tore, on peut définir plus facilement les opérateurs $S_{t}$ par convolution avec un noyau régularisant (voir [Bos86] par exemple). 
Maintenant que l'on a défini des bons espaces de Fréchet, on va définir des "bonnes" applications entre bons espaces de Fréchet. Commençons par rappeler la notion de différentiabilité que l'on va utiliser dans la suite.

Définition C.0.25. Soient $E$ et $F$ des espaces de Fréchet, $U \subseteq E$ un ouvert. Une application $f: U \rightarrow F$ est de classe $C^{1}$ si elle est continue et s'il existe une application continue

$$
\begin{aligned}
& D f: U \times E \quad \longrightarrow \quad F \\
& (x, h) \longmapsto D f(x) . h
\end{aligned}
$$

linéaire en $h$ et telle que pour tout $(x, h) \in U \times E$,

$$
\lim _{t \rightarrow 0} \frac{1}{t}(f(x+t h)-f(x))=D f(x) \cdot h .
$$

Par récurrence, on définit les applications de classe $C^{r}$, pour un entier $r \geq 1$.

On dit encore que $f$ est continûment différentiable au sens de Gâteaux. Il faut remarquer que cette notion est plus générale que la différentiabilité classique dans les espaces de Banach. On peut maintenant définir les bonnes applications.

Définition C.0.26. Soient $E$ et $F$ des bons espaces de Fréchet, $U \subseteq E$ un ouvert. Une application $f: U \rightarrow F$ est bonne si pour tout $x_{0} \in U$, il existe un voisinage $V$ de $x_{0}$, un entier $r>0$ et des réels $c_{j}>0$ telles que pour tout $x \in V$,

$$
\|f(x)\|_{j} \leq c_{j}\left(1+\|x\|_{j+r}\right) .
$$

Une bonne application de classe $C^{r}$ est une application de classe $C^{r}$ qui est bonne ainsi que toutes ses différentielles.

Les inégalités de bonnes applications compensent (en partie) le fait qu'une application continue entre espaces de Banach est automatiquement localement bornée. Cependant l'entier $r$ indique une perte de différentiabilité (par analogie avec le cas où la bonne application est un opérateur différentiel) et les opérateurs de lissage seront alors fondamentaux pour pouvoir utiliser ces inégalités pour des passages à la limite.

Remarque C.0.27. Les bonnes applications et les inégalités qui les définissent sont aussi connus sous le nom d'applications douces et estimations douces, surtout dans le contexte des équations aux dérivées partielles.

Donnons deux résultats couramment utilisés pour s'assurer qu'une application est bonne. On trouvera des preuves dans [Ham82].

Proposition C.0.28. La composée de bonnes applications (de classe $C^{r}$ ) est une bonne application (de classe $C^{r}$ ). 
On définit naturellement les notions de bonnes variétés fréchétiques et de bons groupes de Lie fréchétiques. Dans ce contexte, Dif $f^{\infty}(M)$ est un bon groupe de Lie fréchétique et on a le résultat suivant.

Proposition C.0.29. L'application d'inversion

$$
\begin{array}{ccc}
\mathcal{I}: \operatorname{Diff}^{\infty}(M) & \longrightarrow & \operatorname{Diff}^{\infty}(M) \\
g & \longmapsto & g^{-1}
\end{array}
$$

est un bon difféomorphisme $C^{\infty}$.

On peut alors énoncer le théorème d'inversion locale de Nash, Moser et Hamilton (... entre autres).

Théorème C.0.30 (Nash-Moser-Hamilton). Soient $E$ et $F$ des bons espaces de Fréchet, $U \subseteq E$ un ouvert et $f: U \rightarrow F$ une bonne application de classe $C^{r}, r \geq 2$. Soient $x_{0} \in U$ et $y_{0}=f\left(x_{0}\right)$. On suppose qu'il existe un voisinage $V_{0}$ de $x_{0}$ tel que pour tout $x \in V_{0}$, df $(x)$ soit inversible d'inverse une bonne application linéaire continue. Alors $f$ est un bon difféomorphisme local au voisinage de $x_{0}$.

Donnons une très vague idée de la preuve. On suppose $x_{0}=y_{0}=0$, et on veut résoudre

$$
f(x)=y
$$

où $x$ est l'inconnue et $y$ proche de 0 .

Une preuve du théorème d'inversion locale classique utilisant un algorithme de Newton commencerait comme ceci : notons $L(x)$ l'inverse de $d f(x)$, on considère la suite

$$
\left\{\begin{aligned}
x_{0} & =0 \\
x_{j+1} & =x_{j}-\Delta x_{j}
\end{aligned}\right.
$$

où $\Delta x_{j}=L\left(x_{j}\right)\left(f\left(x_{j}\right)-y\right)$. En estimant la taille des erreurs successives $\left\|\Delta x_{j}\right\|$, on démontre alors la convergence de cette suite et que la limite $x_{\infty}$ résout l'équation.

Cependant, cette méthode ne peut pas fonctionner ici : en effet, grâce aux inégalités de bonnes applications, on ne peut majorer $\left\|\Delta x_{j}\right\|_{k}$ que par des expressions en les $\|y\|_{l}$, avec $l$ arbitrairement grand lorsque $j \rightarrow+\infty$. La convergence de la suite n'est donc pas garantit. Comme on l'a déjà dit plus haut, on va utiliser les opérateurs de lissage pour remédier à ce problème. On considère un algorithme de Newton modifié

$$
\left\{\begin{aligned}
x_{0} & =0 \\
x_{j+1} & =x_{j}-\Delta x_{j}
\end{aligned}\right.
$$



avec $\Delta x_{j}=S\left(t_{j}\right) L\left(x_{j}\right)\left(f\left(x_{j}\right)-y\right)$, et $t_{j}$ qui croît suffisamment vite (typiquement on choisit $t_{j}=\exp \left(\frac{3}{2}\right)^{j}$ ) pour compenser la perte de différentiabilité. La partie technique consiste alors à montrer la convergence de ce procédé. 


\section{Bibliographie}

[AK98] V.I. Arnold and B.A. Khesin, Topological methods in hydrodynamics, Applied Mathematical Sciences, Springer Verlag, NewYork, 1998.

[And58] R.D. Anderson, The algebraic simplicity of certain groups of homeomorphisms, Amer. Jour. Math. 80 (1958), 955-963.

[Ban78] A. Banyaga, Sur la structure du groupe des difféomorphismes qui preservent une forme symplectique, Comment. Math. Helv. 53 (1978), 174-227.

[Ban97]__ The structure of classical diffeomorphism groups, Mathematics and its Applications, Kluwer Academic Publishers Group, Dordrecht, 1997.

[Bin83] R.H. Bing, The geometric topology of 3-manifolds, Colloquium publications, American Mathematical Society, Providence, 1983.

[Bos86] J.-B. Bost, Tores invariants des systemes dynamiques hamiltoniens, Séminaire Bourbaki 133-134 (1986), 113-157.

[Bot70] R. Bott, On a topological obstruction to integrability, Global Analysis, Proc. Sympos. Pure Math. (1970), no. 16, 127-131.

[Bre93] G.E. Bredon, Topology and Geometry, Graduate Texts in Mathematics, Springer Verlag, 1993.

[Cal70] E. Calabi, On the group of automorphisms of a symplectic manifold, Problems in analysis, a symposium in honor of S. Bochner (1970), 1-26.

[EE69] C.J. Earle and J. Eells, A fibre bundle description of Teichmüller theory, J. Differ. Geom. 3 (1969), 19-43.

[EH89] I. Ekeland and H. Hofer, Symplectic topology and hamiltonian dynamics, Math. Z. 200 (1989), no. 3, 355-378.

[EH90]_, Symplectic topology and hamiltonian dynamics II, Math. Z. 203 (1990), no. 4, 553-567.

[EK71] R.D. Edwards and R. Kirby, Deformations of spaces of imbeddings, Ann. of Math. 93 (1971), 63-68. 
[EP93] Y. Eliashberg and L. Polterovich, Bi-invariant metrics on the group of hamiltonian diffeomorphisms, 727-738.

[Eps70] D.B.A. Epstein, The simplicity of certain groups of homeomorphisms, Comment. Math. Helv. 53 (1970), 174-227.

[Eps84] Commutators of $C^{\infty}$ diffeomorphisms, Comment. Math. Helv. 59 (1984), 111-122.

[Fat80a] A. Fathi, Structure of the group of homeomorphisms preserving a good measure on a compact manifold, Ann. Sci. Ec. Norm. Sup 13 (1980), 45-93.

[Fat80b] _ Transformations et homéomorphismes préservant la mesure. Systèmes dynamiques minimaux., Thèse, Université d'Orsay (1980).

[Fis60] G.M. Fisher, On the group of all homeomorphisms of a manifold, Trans. of the Amer. Math. Soc. 97 (1960), 193-212.

[Féj04] J. Féjoz, Démonstration du théorème d'Arnold sur la stabilité dy système planétaire (d'après Herman), Erg. Th. Dyn. Sys. 24 (2004), 1521-1582.

[GG97] J.-M. Gambaudo and E. Ghys, Enlacements asymptotiques, Topology 36-6 (1997), 1355-1379.

[Ghy03] E. Ghys, The group of area preserving diffeomorphisms of a surface, Mini-courses at the conference "Geometry and Foliations", Kyoto, Japan (2003).

[Ghy07] , Talk at the conference "Symplectic topology and measure-preserving dynamical systems", Snowbird, Utah (2007).

[Gro85] M. Gromov, Pseudo holomorphic curves in symplectic manifolds, Invent. Math. 82 (1985), 307-347.

[Hae] A. Haefliger, Naissance des feuilletages, d'Ehresmann-Reeb à Novikov, http ://www.foliations.org, 16p.

[Hae58]_. Structures feuilletées et cohomologie à valeur dans un faisceau de groupoïdes, Comment. Math. Helv. 32 (1958), 248329 .

[Hae71] _ Homotopy and integrability, Manifolds - Amsterdam 1970, Proc. NUFFIC Summer School Manifolds 1970, Lect. Notes Math. 197 (1971), 133-163.

[Hae78] C C Chomology of Lie algebras and foliations, Differ. Topol., Foliations, Gelfand-Fuks Cohomol. ; Proc. Symp., Rio de Janeiro 1976, Lect. Notes Math. 652 (1978), 1-12.

[Hal95] S. Haller, Groups of diffeomorphisms, Master's thesis, University of Vienna, 1995.

[Hal98] _ Perfectness and simplicity of certain groups of diffeomorphisms, Ph.D. thesis, University of Vienna, 1998. 
[Ham82] R.S. Hamilton, The inverse function theorem of Nash and Moser, Bull. of the Amer. Math. Soc. 7-1 (1982), 65-222.

[HD58] E. Dyer and M.-E. Hamstrom, Regular mappings and the space of homeomorphisms on a 2-manifold, Duke Math. Jour. 25 (1958), 521-531.

[Her73] M.R. Herman, Sur le groupe des difféomorphismes du tore, Ann. Inst. Fourier 23-2 (1973), 75-86.

[Her75] _ Sur le groupe des difféomorphismes $\mathbb{R}$-analytiques du tore, Differential topology and geometry, Lecture notes in Math., Springer 484 (1975), 26-32.

[Hir76] M.W. Hirsh, Differential topology, Springer Verlag, New York, 1976.

[Hof90] H. Hofer, On the topological properties of symplectic maps, Proc. Roy. Soc. Edinburgh Sect. A 115 (1990), no. 1-2, 25-38.

[Hof93] _ Estimates for the energy of a symplectic map, Comment. Math. Helv. 68 (1993), no. 1, 48-72.

[HZ90] H. Hofer and E. Zehnder, A new capacity for symplectic manifolds, Analysis, et cetera, Res. Pap. in Honor of J. Moser's 60th Birthd., 405-427, 1990.

[HZ94] , Symplectic invariants and hamiltonian dynamics, Birkhäuser Advanced Texts Verlag, Basel, 1994.

[KN63] S. Kobayashi and K. Nomizu, Foundations of differential geometry I, Interscience Publishers, New York-London, 1963.

[Lan95] S. Lang, Introduction to diophantine approximations, Springer Verlag, New-York, 1995.

[Lan02] Algebra, Graduate Texts in Mathematics 211, Springer Verlag, New-York, 2002.

[Law77] B. Lawson, The qualitative theory of foliations, A.M.S. Series 27, A.M.S., Providence R.I., 1977.

[LM95] F. Lalonde and D. McDuff, The geometry of symplectic energy, Ann. of Math. 149 (1995), no. 2, 349-371.

[Mas80] W.S. Massey, Singular Homology Theory, Graduate Texts in Mathematics, Springer Verlag, 1980.

[Mat73] J.N. Mather, Integrability in codimension 1, Comment. Math. Helv. 48 (1973), 195-233.

[Mat74a] Commutators of diffeomorphisms I, Comment. Math. Helv. 49 (1974), 512-528.

[Mat74b] _ Simplicity of certain groups of diffeomorphisms, Bull. of the Amer. Math. Soc. 80 (1974), 271-273.

[Mat75] Commutators of diffeomorphisms II, Comment. Math. Helv. 50 (1975), 33-40. 
[Mat79] _ On the homology of Haefliger's classifying spaces, Differential topology, C.I.M.E., Varenna 1976 (1979), 73-116.

[Mat84] A curious remark concerning the geometry of the transfer map, Comment. Math. Helv. 59 (1984), 86-110.

[Mat85] Commutators of diffeomorphisms III : a group which is not perfect, Comment. Math. Helv. 60 (1985), 122-124.

[May92] J.P. May, Simplicial objects in algebraic topology, Chicago Lectures in Mathematics, 1992.

[McD82] D. McDuff, The local homology of groups of volume preserving diffeomorphisms I, Ann. Sci. Ec. Norm. Sup 4 (1982), no. 4, 609-648.

[Mil84] J. Milnor, Remarks on infinite-dimensional Lie groups, Relativité, groupes et topologie II, Les Houches Ec. d'Eté Phys. Théor., Sess. 40 (1984), 1007-1057.

[Mül07] S. Müller, The group of hamiltonian homeomorphisms in the $L^{\infty}$ norm, Preprint (2007).

[Moi77] E.E. Moise, Geometric topology in dimension 2 and 3, Graduate Texts in Mathematics, Springer Verlag, 1977.

[Mos65] J. Moser, On the volume element on a manifold, Trans. of the Amer. Math. Soc. 120 (1965), 286-294.

[Mos66] _ A rapidly convergent iteration method and non-linear differential equations, Ann. Scuola Norm. Sup. Pisa 20 (1966), no. 3, 499-535.

[MS98] D. McDuff and D. Salamon, Introduction to symplectic topology, Oxford University Press, Oxford, 1998.

[Nas56] J. Nash, The embedding problem for riemannian manifolds, Ann. of Math. 63 (1956), no. 2, 20-63.

[Oh06] Y.-G. Oh, The group of hamiltonian homeomorphisms and continuous hamiltonian flows, Preprint (2006).

[OM07] S. Müller and Y.-G. Oh, The group of hamiltonian homeomorphism and $C^{0}$-symplectic topology, J. Symp. Geom. 5 (2007), no. 2, 167-219.

[Ono06] K. Ono, Floer-Novikov cohomology and the flux conjecture, Geom. Funct. Anal. 16-5 (2006), 981-1020.

[OU41] J.C. Oxtoby and S.M. Ulam, Measure-preserving homeomorphisms and metrical transitivity, Ann. of Math. 42 (1941).

[Pol98] L. Polterovich, Hofer's diameter and Lagrangian intersections, Int. Math. Res. Not. (1998), no. 4, 217-223.

[Pol01] The geometry of the group of symplectic diffeomorphisms, Lectures in Mathematics ETH Zürich, Birhäuser Verlag, Basel, 2001. 
[PS70] J. Palis and S. Smale, Structural stability theorems, Global Analysis, Proc. Sympos. Pure Math. 14 (1970), 223-231.

[Rud91] W. Rudin, Functional analysis, International Series in Pure and Applied Mathematics, McGraw-Hill, New York, 1991.

[Sch57] S. Schwartzman, Asymptotic cycles, Ann. of Math. 66-2 (1957), 270-284.

[Ser72] F. Sergeraert, Un théorème de fonctions implicites sur certains espaces de Fréchet et quelques applications, Ann. Sci. Ec. Norm. Sup (1972), 599-660.

[Ser78] — B — Séminaire Bourbaki 20 (1977-1978), 16p.

[Sie05] L. Siebenmann, The Osgood-Schoenflies theorem revisited, Russian Math. Survey 60-4 (2005), 645-672.

[Sik07] J.-C. Sikorav, Approximation of a volume-preserving homeomorphism by a volume-preserving diffeomorphism, Preprint (2007).

[Thu74a] W.P. Thurston, Foliations and group of diffeomorphisms, Bull. of the Amer. Math. Soc. 80 (1974), 304-307.

[Thu74b] , The theory of foliations of codimension greater than one, Comment. Math. Helv. 49 (1974), 214-231.

[Thu76] _ Existence of codimension-one foliations, Ann. of Math. 104 (1976), no. 2, 249-268.

[Tsu] T. Tsuboi, Classifying spaces for groupoid structures, http ://www.foliations.org, 14p.

[Vit89] C. Viterbo, Capacités symplectiques et applications, Séminaire Bourbaki 177-178 (1989), 345-362.

[Vit06] - On the uniqueness of generating hamiltonian for continuous limits of hamiltonians flows, Int. Math. Res. Not. (2006), 9 pp.

\author{
Abed Bounemoura \\ Département de Mathématiques \\ Bâtiment 425 \\ Faculté des Sciences d'Orsay \\ Université Paris-Sud 11 \\ F-91405 Orsay Cedex \\ Abed.Bounemoura@math.u-psud.fr
}

